\title{
Quarterly Coal Report
}

\section{April-June 1998}

November 1998

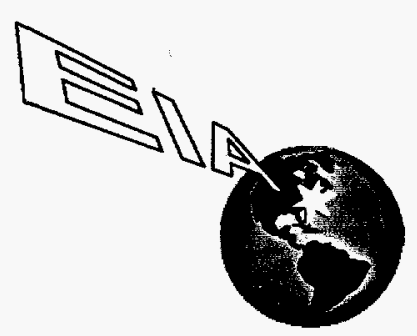

RECEIVED NOV 251998

OSTI 


\section{HOW TO OBTAIN EIA PRODUCTS AND SERVICES}

For further information on any of the following services, or for answers to energy information questions, please contact EIA's National Energy Information Center:

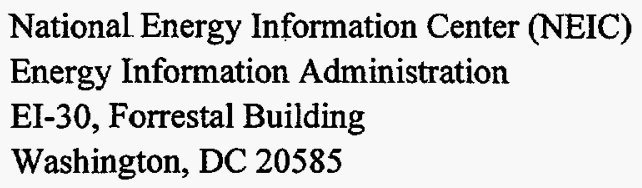

(202) $586-8800$

(202) 586-0727 (fax)

TTY: (202) 586-1181

E-mail: infoctr@eia.doe.gov

\section{Electronic Products and Services}

EIA's Internet Site Services offer nearly all EIA publications. Users can view and download selected pages or entire reports, search for information, download EIA data and analysis applications, and find out about new EIA information products and services.

World Wide Web: http://www.eia.doe.gov

FTP: ftp://ftp.eia.doe.gov

EIA also offers a listserve service for EIA press releases and other short documents. Sign up on the EIA World Wide Web site.

EIA's CD-ROM, Energy InfoDisc, contains most EIA publications and major energy database applications. The Energy InfoDisc, produced quarterly, is available for a fee from STAT-USA, Department of Commerce, 1-800-STAT-USA.

The Comprehensive Oil and Gas Information Source (COGIS), a bulletin board service, contains data files from most of EIA's oil-and gas-related reports. It is available for a fee from STAT-USA, on 1-800-STAT-USA.

\section{Printed Publications}

EIA directories are available free of charge from NEIC. Recent periodicals and one-time reports are available from the Government Printing Office. Older reports are available from the National Technical Information Service:

\section{Superintendent of Documents}

U.S. Government Printing Office

P.O. Box 371954

Pittsburgh, PA 15250-7954

(202) 512-1800; (202)-512-2250 (fax)

\author{
National Technical Information Service \\ U.S. Department of Commerce \\ Springfield, VA 22161 \\ 5285 Port Royal Road \\ 1-(800) 553-6847; (703) 321-8547 (fax)
}

Released for Printing: November 5, 1998

Printed with soy ink on recycled paper

Questions concerning the contents of this report should be directed to Paulette Young, (202) 426-1150.

The Quarterly Coal Report (ISSN 0736-4598) is published quarterly by the Energy Information Administration, 1000 Independence Avenue, SW, Washington, DC 20585 , and sells for $\$ 22.00$ per year (price subject to change without advance notice). Second-class postage paid at Washington, DC 20066-9998, and additional mailing offices. POSTMASTER: Send address changes to Quarterly Coal Report, Energy Information Administration, El-30, 1000 Independence Avenue, SW, Washington, DC 20585. 


\title{
Quarterly Coal Report April-June 1998
}

November 1998

\author{
Energy Information Administration \\ Office of Coal, Nuclear, Electric \\ and Alternate Fuels \\ U.S. Department of Energy \\ Washington, DC 20585
}

DISTRIBUTION OF THIS DOCUMENT IS UNMMTED f NAS

This report was prepared by the Energy Information Administration, the independent statistical and analytical agency within the Department of Energy. The information contained herein should not be construed as advocating or reflecting any policy position of the Department of Energy or any other organization. 


\section{Contacts}

This publication was prepared by Paulette Young under the direction of Mary K. Paull, Project Leader, Coal Data Branch, Coal and Electric Data and Renewables Division, Office of Coal, Nuclear, Electric and Alternate Fuels. Questions addressing the Appendix A, U.S. Coal Imports section should be directed to Paulette Young at (202) 426-1150, email PYOUNG@EIA.DOE.GOV. All other questions on coal statistics should be directed to the National Energy Information Center (NEIC) at (202) 586-8800, email INFOCTR@EIA.DOE.GOV. 


\section{DISCLAIMER}

This report was prepared as an account of work sponsored by an agency of the United States Government. Neither the United States Government nor any agency thereof, nor any of their employees, makes any wartanty, express or implied, or assumes any legal liability or responsibility for the accuracy, completeness, or usefulness of any information, apparatus, product, or process disclosed, or represents that its use would not infringe privately owned rights. Reference herein to any specific commercial product, process, or service by trade name, trademark, manufacturer, or otherwise does not necessarily constitute or imply its endorsement, recommendation, or favoring by the United States Government or any agency thereof. The views and opinions of authors expressed herein do not necessarily state or reflect those of the United States Government or any agency thereof. 


\section{DISCLAIMER}

Portions of this document may be illegible in electronic image products. Images are produced from the best available original document. 


\section{Preface}

The Quarterly Coal Report (QCR) provides comprehensive information about U.S. coal production, distribution, exports, imports, receipts, prices, consumption, and stocks to a wide audience, including Congress, Federal and State agencies, the coal industry, and the general public. Coke production, consumption, distribution, imports, and exports data are also provided. The data presented in the QCR are collected and published by the Energy Information Administration (EIA) to fulfill data collection and dissemination responsibilities as specified in the Federal Energy Administration Act of 1974 (Public Law 93-275), as amended.

This report presents detailed quarterly data for April through June 1998 and aggregated quarterly historical data for 1992 through the first quarter of 1998. Appendix A displays, from 1992 on, detailed quarterly historical coal imports data, as specified in Section 202 of the Energy Policy and Conservation Amendments Act of 1985 (Public Law 99-58).

To provide a complete picture of coal supply and demand in the United States, historical information has been integrated in this report. Additional historical data can also be found in the following EIA publications :

Annual Energy Review 1997 DOE/EIA-0384(98); Monthly Energy Review DOE/EIA-0035; Coal Data: $A$ Reference DOE/EIA-0064(93) DOE/EIA-0035; and Coal Industry Annual DOE/EIA-0584(96) .

The historical data in this report are collected by the EIA in three quarterly coal surveys (coal consumption at manufacturing plants, coal production, and coal consumption at coke plants), one annual coal production survey, and two monthly surveys of electric utilities. The coal surveys originated in the 1920 's, at the Bureau of Mines, U.S. Department of the Interior. In 1977 , the responsibility for these surveys was transferred to the EIA under the Department of Energy Organization Act (Public Law 95-91). The two electric utility surveys originated at the Federal Power Commission (FPC)--one in 1936 under the Federal Power Act and one in 1972 under FPC Order Number 453. The EIA continued these surveys, reducing the frequency and quantity of information requested and increasing the automation of the associated data processing and report generation functions. Coal export and import data are obtained from the Bureau of the Census, U.S. Department of Commerce, which compiles monthly data from documents filed with the U.S. Customs Service, as required by law.

All data for 1998 are preliminary. All data for 1997 are final. U.S coal production data for 1997 and previous years are based on the annual survey Form EIA-7A, "Coal Production Report." Coal production data for 1998 are preliminary and are based on the quarterly survey, Form 7000-2, "Quarterly Mine Employment and Coal Production Report," U.S. Department of Labor, Mine Safety and Health Administration.

All coke tables have been modified to reflect the closing of several coke plants in 1997.

The Office of Coal, Nuclear, Electric and Alternate Fuels acknowledges the cooperation of the respondents in supplying the information published in this report. 



\section{Contents}

\section{Page}

Production 3

Exports and Imports

Receipts

Consumption

Stocks

Appendices

A. U.S. Coal Imports 


\section{Tables}

Page

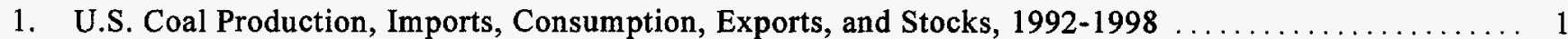

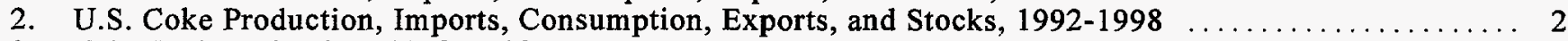

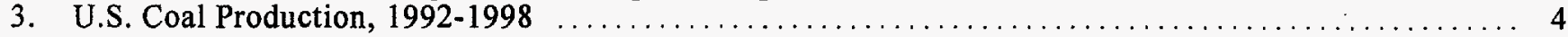

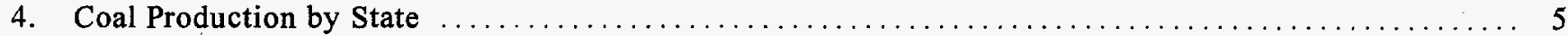

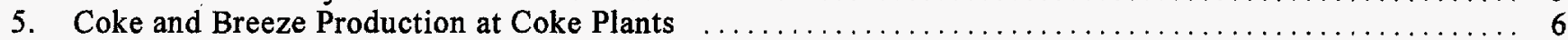

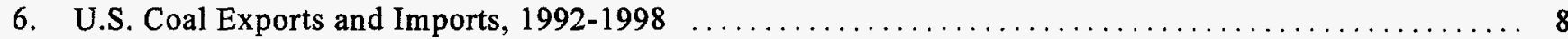

7. Average Price of U.S. Coal Exports and Imports, $1992-1998 \ldots \ldots \ldots \ldots \ldots \ldots \ldots \ldots \ldots \ldots \ldots \ldots . \ldots$

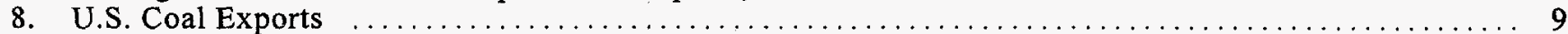

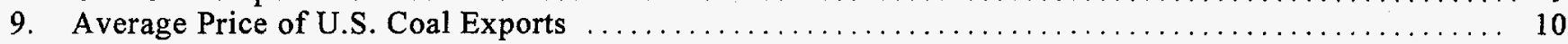

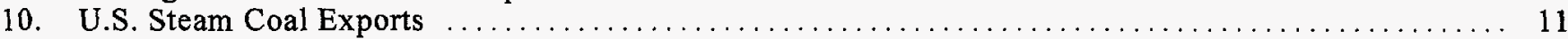

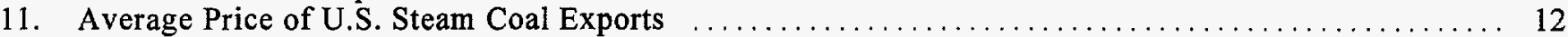

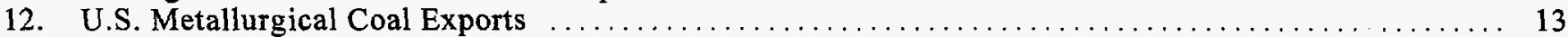

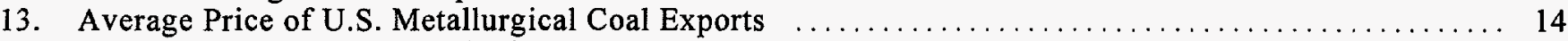

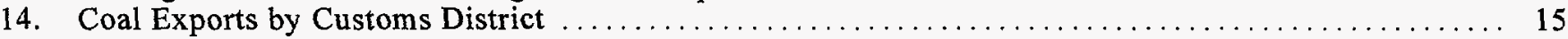

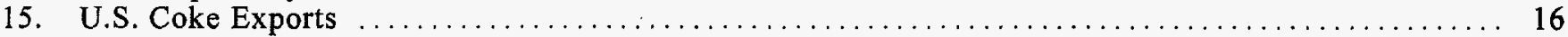

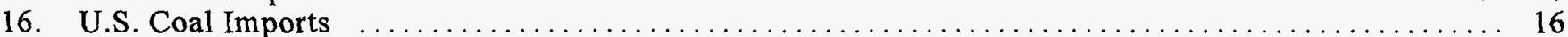

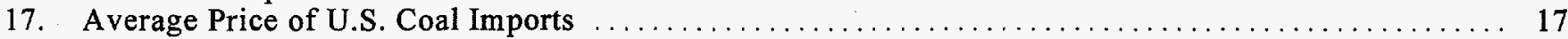

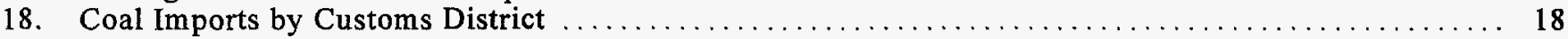

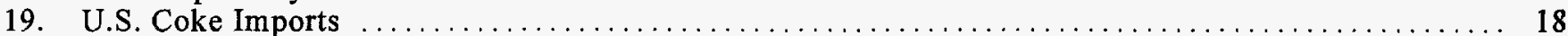

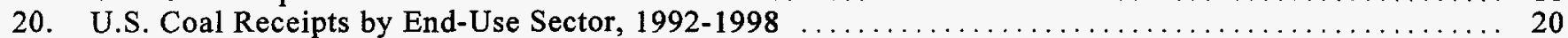

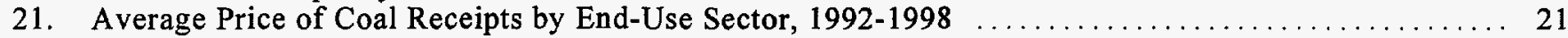

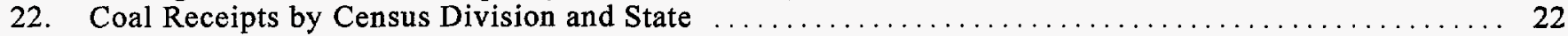

23. Quantity and Price of Coal Receipts at Electric Utility Plants by Census Division and State .......... 23

24. Quantity and Price of Contract Coal Receipts at Electric Utility Plants by Census Division and State .. 24

25. Quantity and Price of Spot Coal Receipts at Electric Utility Plants by Census Division and State . .... 25

26. Average Cost of Coal Receipts at Electric Utility Plants by Census Division and State . . ......... 26

27. Coal Receipts and Prices by Sulfur Content at Electric Utility Plants, by State of Origin and Imports,

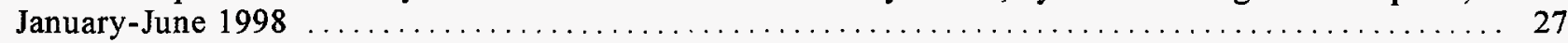

28. Destination of Coal Received at Electric Utility Plants by Origin, January-June 1998, $1997 \ldots \ldots \ldots 28$

29. Origin of Coal Received at Electric Utility Plants by Destination, January-June 1998, $1997 \ldots \ldots \ldots \ldots 32$

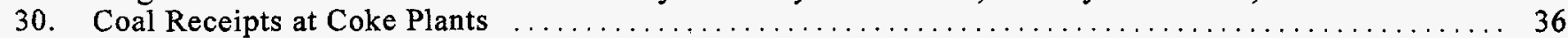

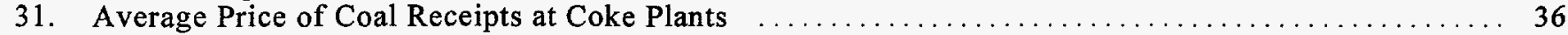

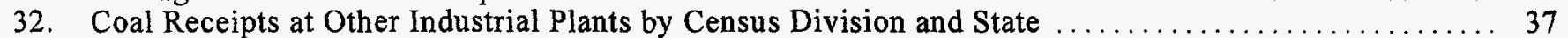

33. Average Price of Coal Receipts at Other Industrial Plants by Census Division and State ............ 38

34. U.S. Coal Receipts at Manufacturing Plants by Standard Industrial Classification (SIC) Code ....... 39

35. Average Price of U.S. Coal Receipts at Manufacturing Plants by Standard Industrial Classification (SIC) Code

Coal Receipts by the Residential and Commercial Sector by Census Division and State . . ........ 40

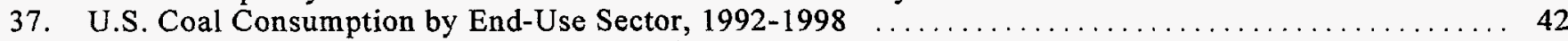

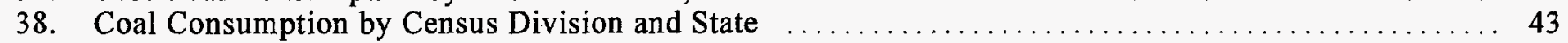

39. Coal Consumption at Electric Utility Plants by Census Division and State $\ldots \ldots \ldots \ldots \ldots \ldots \ldots \ldots \ldots \ldots \ldots$

40. Change in Electric Utility Net Generation by State and Census Division, 1998 versus $1997 \ldots \ldots \ldots .45$

41. Coal Carbonized at Coke Plants by Census Division and State $\ldots \ldots \ldots \ldots \ldots \ldots \ldots \ldots \ldots \ldots \ldots$

42. Coal Consumption at Other Industrial Plants by Census Division and State $\ldots \ldots \ldots \ldots \ldots \ldots \ldots \ldots \ldots \ldots$

43. U.S. Coal Consumption at Manufacturing Plants by Standard Industrial Classification (SIC) Code $\ldots . .48$

44. Coal Consumption by Residential and Commercial Sector by Census Division and State . . . . . . . . . 49

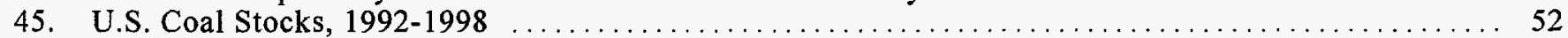

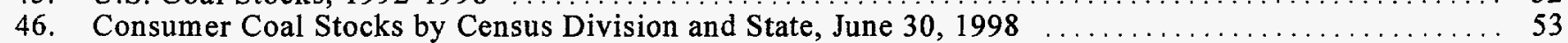

47. Coal Stocks at Electric Utility Plants by Census Division and State $\ldots \ldots \ldots \ldots \ldots \ldots \ldots \ldots \ldots \ldots \ldots$

48. Coal Stocks at Coke Plants by Census Division and State $\ldots \ldots \ldots \ldots \ldots \ldots \ldots \ldots \ldots \ldots \ldots \ldots \ldots$

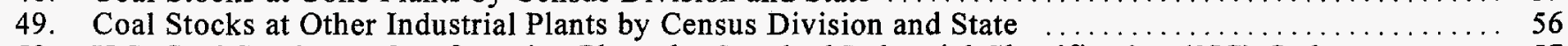

50. U.S. Coal Stocks at Manufacturing Plants by Standard Industrial Classification (SIC) Code $\ldots \ldots \ldots \ldots \ldots$

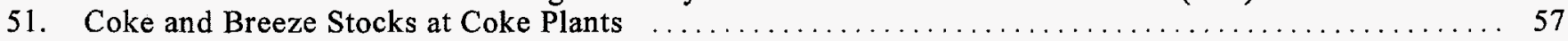

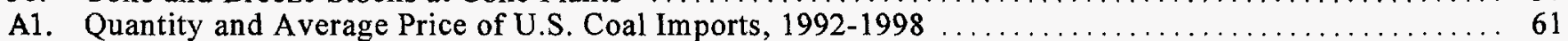

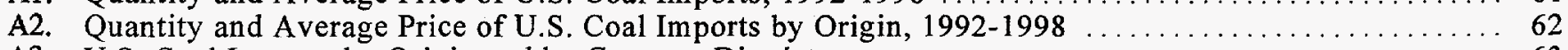

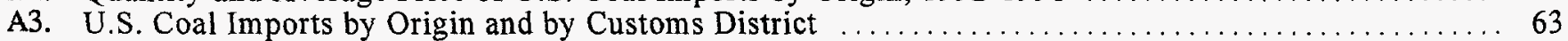


A4. Average Price of U.S. Coal Imports by Origin and by Customs District

A5. Imported Coal Received at Electric Utility Plants by Origin

A6. Cost and Quality of Imported Coal Received at Electric Utility Plants by Origin, 1992-1998 . . . ... 66

A7. Cost and Quality of All Coal Received at Electric Utility Plants that Import Coal by Origin, 1992-1998 74 
Table 1. U.S. Coal Production, Imports, Consumption, Exports, and Stocks, 1992-1998 (Thousand Short Tons)

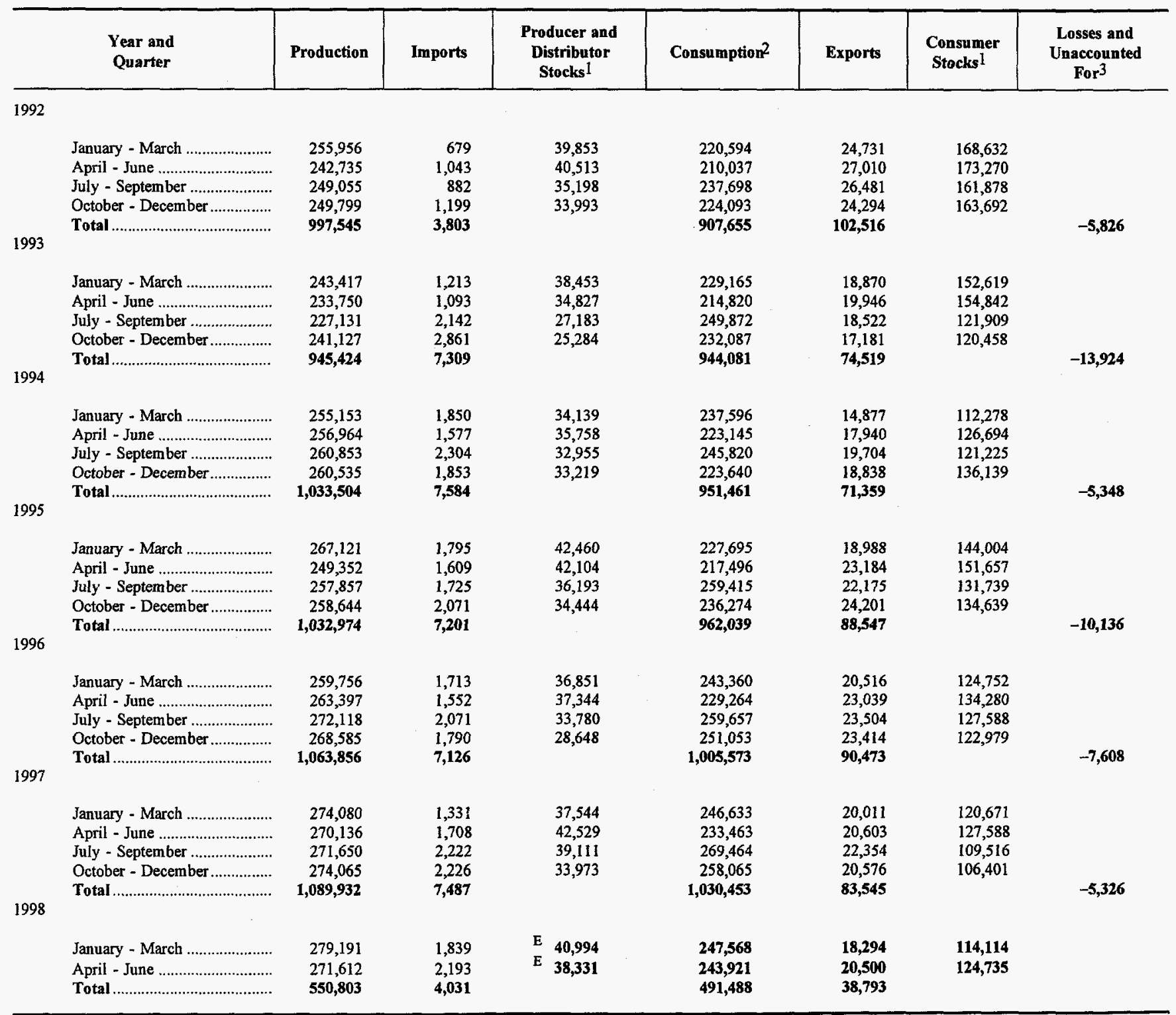

1 Reported as of the last day of the quarter.

2 Total/Annual includes coal consumed by independent power producers to generate electricity and cogeneration plants not included in the other industrial, coke and commercial sectors. Therefore, quarterly data does not total to Total/Annual data.

3 Losses and Unaccounted For is calculated as production plus imports minus the change in producer and distributor stocks minus consumption minus exports minus the change in consumer stocks.

Estimated.

Notes: Consumption data for 1992 through 1997 includes coal consumed by independent power producers to generate electricity and cogeneration plants not included in the other industrial, coke, and commercial sectors. Total may not equal sum of components because of independent rounding.

Sources: - Production: Energy Information Administration (EIA), Form EIA-6, Schedule Q, "Quarterly Coal Report;" and Form EIA-7A, "Coal Production Report"; Mine Safety and Health Administration, U.S. Department of Labor, Form 7000-2, "Quarterly Mine Employment and Coal Production Report," and State mining agency coal production reports; - Imports: Bureau of the Census, U.S. Department of Commerce, "Monthly Report IM 145" - Producer and Distributor Stocks: EIA, Form EIA-6, "Coal Distribution Report;" and Form EIA-6, Schedule Q, "Quarterly Coal Report;" - Exports: Bureau of the Census, U.S. Department of Commerce, "Monthly Report EM 545" - Consumption and Consumer Stocks: EIA, Form EIA-759, "Monthly Power Plant Report;" Form EIA-3, "Quarterly Coal Consumption Report - Manufacturing Plants;" Form EIA-5, "Coke Plant Report - Quarterly;" Form ELA-867, "Annual Nonutility Power Producer Report;" Form EIA-7A, "Coal Production Report;" and Form EIA-6, "Coal Distribution Report." 
Table 2. U.S. Coke Production, Imports, Consumption, Exports, and Stocks, 1992-1998 (Thousand Short Tons)

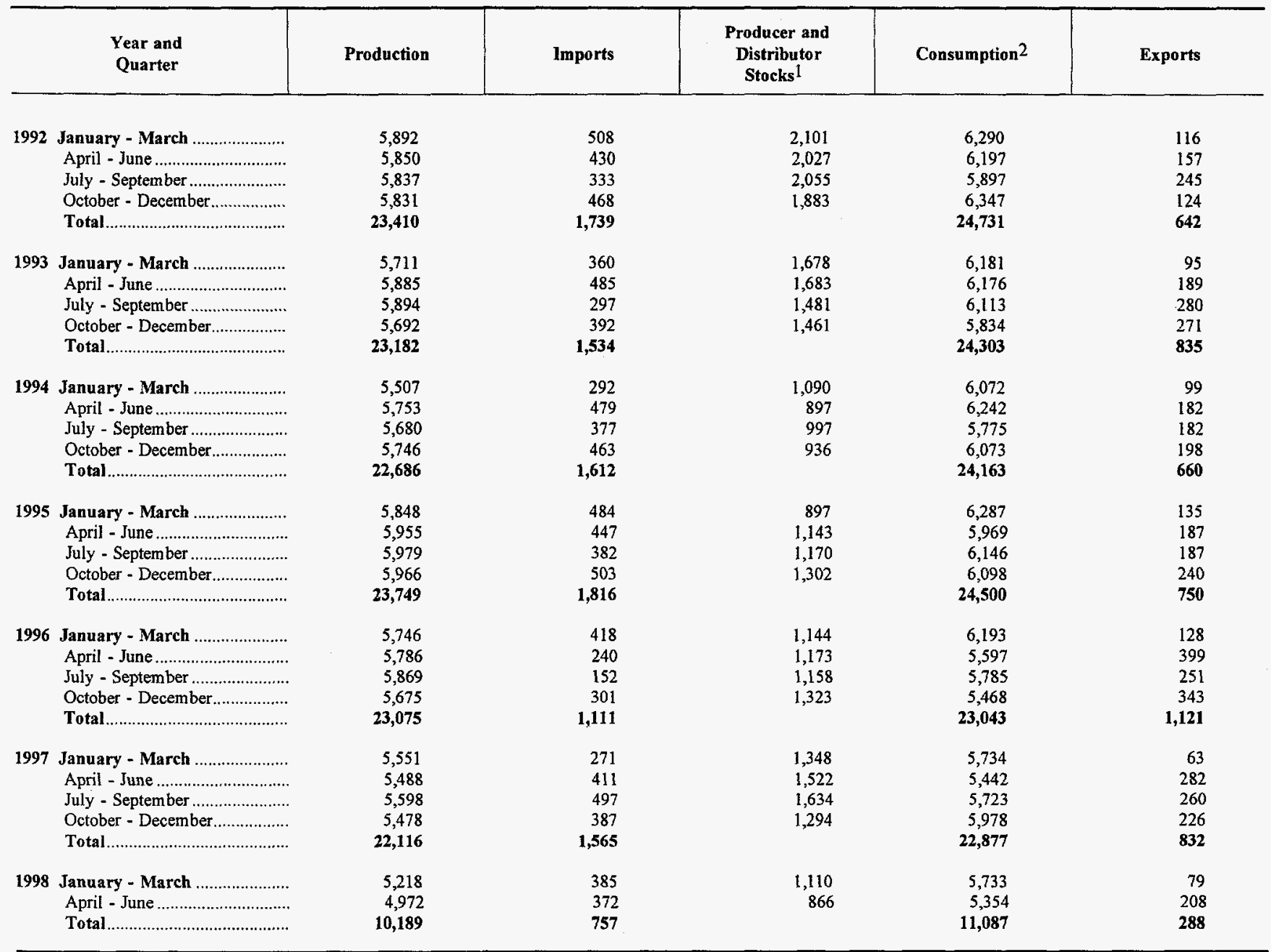

1 Reported as of the last day of the quarter.

2 Consumption is equal to production plus imports plus/minus the change in producer and distributor stocks minus exports.

Notes: Total may not equal sum of components because of independent rounding.

Sources: - Production, Consumption, and Producer and Distributor Stocks: Energy Information Administration, Form EIA-5, "Coke Plant Report -

Quarterly" - Imports: Bureau of the Census, U.S. Department of Commerce, "Monthly Report IM 145" and - Exports: Bureau of the Census, U.S. Department of Commerce, "Monthly Report EM 545." 


\section{Production}


Table 3. U.S. Coal Production, 1992-1998

(Thousand Short Tons)

\begin{tabular}{|c|c|c|c|c|c|}
\hline Year & January - March & April - June & July - September & October - December & Year to Date \\
\hline 1992 & 255,956 & 242,735 & 249,055 & 249,799 & 997,545 \\
\hline 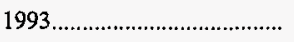 & 243,417 & 233,750 & 227,131 & 241,127 & 945,424 \\
\hline 1994 & 255,153 & 256,964 & 260,853 & 260,535 & $1,033,504$ \\
\hline 1995 & 267,121 & 249,352 & 257,857 & 258,644 & $1,032,974$ \\
\hline 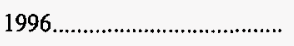 & 259,756 & 263,397 & 272,118 & 268,585 & $1,063,856$ \\
\hline 1997 & 274,080 & 270,136 & 271,650 & 274,065 & $1,089,932$ \\
\hline $1998 \ldots$ & 279,191 & 271,612 & NA & NA & 550,803 \\
\hline
\end{tabular}

NA Not available.

Notes: Total may not equal sum of components because of independent rounding.

Sources: Energy Information Administration (EIA), Form EIA-6, Schedule Q, "Quarterly Coal Report;" and Form EIA-7A, "Coal Production Report;" Mine Safety and Health Administration, U.S. Department of Labor, Form 7000-2, "Quarterly Mine Employment and Coal Production Report;" and State mining agency coal production reports. 
Table 4. Coal Production by State

(Thousand Short Tons)

\begin{tabular}{|c|c|c|c|c|c|c|}
\hline \multirow{2}{*}{$\begin{array}{l}\text { Coal-Producing Region } \\
\text { and State }\end{array}$} & \multirow{2}{*}{$\begin{array}{c}\text { April - } \\
\text { June } \\
1998\end{array}$} & \multirow{2}{*}{$\begin{array}{c}\text { January - } \\
\text { March } \\
1998\end{array}$} & \multirow{2}{*}{$\begin{array}{l}\text { April - } \\
\text { June } \\
1997\end{array}$} & \multicolumn{3}{|c|}{ Year to Date } \\
\hline & & & & 1998 & 1997 & $\begin{array}{l}\text { Percent } \\
\text { Change }\end{array}$ \\
\hline Alabama & 5,647 & 6,382 & 6,528 & 12,029 & 13,042 & -7.8 \\
\hline Alaska & 393 & 367 & 318 & 760 & 690 & 10.1 \\
\hline 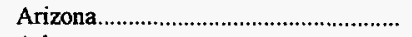 & 2,616 & 3,136 & 2,629 & 5,752 & 5,605 & 2.6 \\
\hline 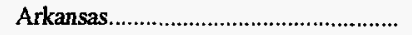 & 8 & 7 & 4 & 16 & 8 & 93.5 \\
\hline Colorado & 7,424 & 7,034 & 7,941 & 14,458 & 15,061 & -4.0 \\
\hline 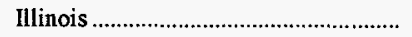 & 10,603 & 8,995 & 10,211 & 19,598 & 22,107 & -11.4 \\
\hline 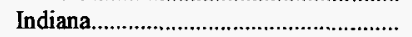 & 8,996 & 9,219 & 8,933 & 18,215 & 16,197 & 12.5 \\
\hline 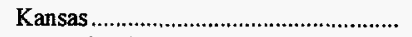 & 97 & 94 & 114 & 191 & 197 & -2.8 \\
\hline 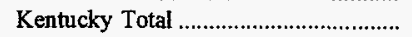 & 33,988 & 39,331 & 38,537 & 73,319 & 77,845 & -5.8 \\
\hline 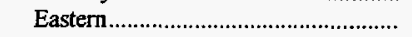 & 26,539 & 30,755 & 30,659 & 57,294 & 61,438 & -6.7 \\
\hline Western & 7,449 & 8,576 & 7,878 & 16,025 & 16,408 & -2.3 \\
\hline 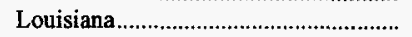 & 736 & 750 & 971 & 1,486 & 1,763 & -15.7 \\
\hline Maryland & 912 & 1,111 & 945 & 2,022 & 2,121 & -4.6 \\
\hline 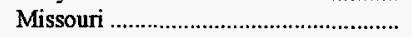 & 82 & 73 & 85 & 155 & 214 & -27.4 \\
\hline Montana & 10,638 & 10,455 & 9,234 & 21,093 & 18,948 & 11.3 \\
\hline New Mexico & 6,820 & 6,622 & 6,613 & 13,442 & 13,722 & -2.0 \\
\hline 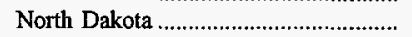 & 6,911 & 7,794 & 6,965 & 14,705 & 14,409 & 2.1 \\
\hline Ohio & 7,845 & 7,595 & 6,809 & 15,440 & 14,293 & 8.0 \\
\hline 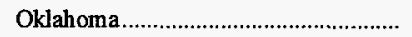 & 457 & 416 & 395 & 873 & 822 & 6.2 \\
\hline 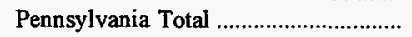 & 18,471 & 19,606 & 19,589 & 38,077 & 37,633 & 1.2 \\
\hline Anthracite & 952 & 921 & 1,058 & 1,873 & 2,206 & -15.1 \\
\hline 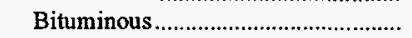 & 17,519 & 18,686 & 18,531 & 36,204 & 35,427 & 2.2 \\
\hline 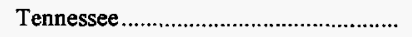 & 659 & 656 & 833 & 1,315 & 1,768 & -25.6 \\
\hline 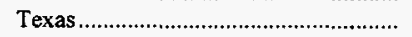 & 13,081 & 12,823 & 12,462 & 25,904 & 25,796 & .4 \\
\hline Utah & 5,808 & 6,367 & 7,258 & 12,175 & 13,889 & -12.3 \\
\hline 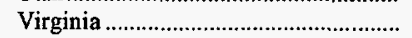 & 8,160 & 8,301 & 9,037 & 16,460 & 18,219 & -9.7 \\
\hline 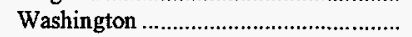 & 1,191 & 894 & 976 & 2,085 & 1,780 & 17.1 \\
\hline 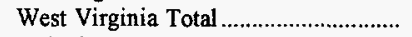 & 43,348 & 44,742 & 44,275 & 88,089 & 89,845 & -2.0 \\
\hline 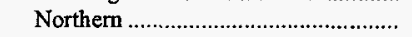 & 11,689 & 12,761 & 10,809 & 24,450 & 22,672 & 7.8 \\
\hline Southern & 31,659 & 31,980 & 33,466 & 63,640 & 67,173 & -5.3 \\
\hline 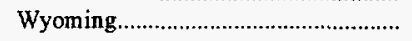 & 76,723 & 76,421 & 68,473 & 153,144 & 138,241 & 10.8 \\
\hline Appalachian Total ................................. & 111,580 & 119,148 & 118,675 & 230,728 & 238,358 & -3.2 \\
\hline Interior Total & 41,509 & 40,953 & 41,054 & 82,462 & $\mathbf{8 3 , 5 1 1}$ & -1.3 \\
\hline 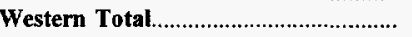 & 118,523 & 119,090 & 110,407 & 237,613 & 222,347 & 6.9 \\
\hline East of the Miss. River ............................ & 138,628 & 145,938 & 145,698 & 284,566 & 293,070 & -2.9 \\
\hline 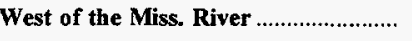 & 132,984 & 133,253 & 124,438 & 266,237 & 251,146 & 6.0 \\
\hline U.S. Total & 271,612 & 279,191 & 270,136 & $\mathbf{5 5 0 , 8 0 3}$ & 544,216 & 1.2 \\
\hline
\end{tabular}

Notes: Total may not equal sum of components because of independent rounding.

Sources: Energy Information Administration (EIA), Form EIA-6, Schedule Q, "Quarterly Coal Report;" and Form EIA-7A, "Coal Production Report;" Mine Safety and Health Administration, U.S. Department of Labor, Form 7000-2, "Quarterly Mine Employment and Coal Production Report," and State mining agency coal production reports. 
Table 5. Coke and Breeze Production at Coke Plants

(Thousand Short Tons)

\begin{tabular}{|c|c|c|c|c|c|c|}
\hline \multirow[b]{2}{*}{ Census Division } & \multirow{2}{*}{$\begin{array}{l}\text { April - } \\
\text { June } \\
1998\end{array}$} & \multirow{2}{*}{$\begin{array}{c}\text { January - } \\
\text { March } \\
1998\end{array}$} & \multirow{2}{*}{$\begin{array}{l}\text { April - } \\
\text { June } \\
1997\end{array}$} & \multicolumn{3}{|c|}{ Year to Date } \\
\hline & & & & 1998 & 1997 & $\begin{array}{l}\text { Percent } \\
\text { Change }\end{array}$ \\
\hline Middle Atlantic Total. & 1,628 & 2,046 & 2,254 & 3,674 & 4,474 & -17.9 \\
\hline 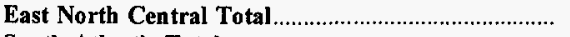 & 2,148 & 2,119 & 2,182 & 4,267 & 4,409 & -3.2 \\
\hline South Atlantic Total & w & w & w & w & $\mathbf{w}$ & $\mathbf{w}$ \\
\hline East South Central Total & 764 & 717 & 851 & 1,481 & 1,726 & -14.2 \\
\hline Mountain Total. & w & w & w & w & w & $\mathbf{w}$ \\
\hline U.S. Total & 5,277 & 5,525 & 5,798 & 10,803 & 11,660 & -7.4 \\
\hline 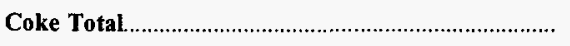 & 4,972 & 5,218 & 5,488 & 10,189 & 11,040 & -7.7 \\
\hline 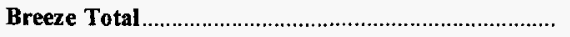 & 305 & 308 & 309 & 613 & 621 & -1.2 \\
\hline
\end{tabular}

w Withheld to avoid disclosure of individual company data.

Note: Total may not equal sum of components because of independent rounding.

Source: Energy Information Administration, Form EIA-5, "Coke Plant Report - Quarterly." 


\section{Exports and Imports}


Table 6. U.S. Coal Exports and Imports, 1992-1998

(Thousand Short Tons)

\begin{tabular}{|c|c|c|c|c|c|c|c|c|c|c|}
\hline \multirow{2}{*}{ Year } & \multicolumn{2}{|c|}{ January - March } & \multicolumn{2}{|c|}{ April - June } & \multicolumn{2}{|c|}{ July - September } & \multicolumn{2}{|c|}{ October - December } & \multicolumn{2}{|c|}{ Year to Date } \\
\hline & Exports & Imports & Exports & Imports & Exports & Imports & Exports & Imports & Exports & Imports \\
\hline $1992 \ldots \ldots \ldots \ldots \ldots$ & 24,731 & 679 & 27,010 & 1,043 & 26,481 & 882 & 24,294 & 1,199 & 102,516 & 3,803 \\
\hline $1993 \ldots \ldots \ldots \ldots \ldots \ldots \ldots$ & 18,870 & 1,213 & 19,946 & 1,093 & 18,522 & 2,142 & 17,181 & 2,861 & 74,519 & 7,309 \\
\hline $1994 \ldots \ldots \ldots \ldots \ldots \ldots$ & 14,877 & 1,850 & 17,940 & 1,577 & 19,704 & 2,304 & 18,838 & 1,853 & 71,359 & 7,584 \\
\hline 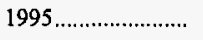 & 18,988 & 1,795 & 23,184 & 1,609 & 22,175 & 1,725 & 24,201 & 2,071 & 88,547 & 7,201 \\
\hline $1996 \ldots \ldots \ldots \ldots \ldots \ldots$ & 20,516 & 1,713 & 23,039 & 1,552 & 23,504 & 2,071 & 23,414 & 1,790 & 90,473 & 7,126 \\
\hline $1997 \ldots \ldots \ldots \ldots \ldots \ldots$ & 20,011 & 1,331 & 20,603 & 1,708 & 22,354 & 2,222 & 20,576 & 2,226 & 83,545 & 7,487 \\
\hline $1998 \ldots \ldots \ldots \ldots \ldots$ & 18,294 & 1,839 & 20,500 & 2,193 & NA & NA & NA & NA & 38,793 & 4,032 \\
\hline
\end{tabular}

NA Not available.

Notes: Total may not equal sum of components because of independent rounding. More detailed data included in Table A3.

Sources: Exports: Bureau of the Census, U.S. Department of Commerce, "Monthly Report EM 545;" and Imports: Bureau of the Census, U.S. Department of Commerce, "Monthly Report IM 145."

Table 7. Average Price of U.S. Coal Exports and Imports, 1992-1998 (Dollars per Short Ton)

\begin{tabular}{|c|c|c|c|c|c|c|c|c|c|c|}
\hline \multirow{2}{*}{ Year } & \multicolumn{2}{|c|}{ January - March } & \multicolumn{2}{|c|}{ April - June } & \multicolumn{2}{|c|}{ July - September } & \multicolumn{2}{|c|}{ October - December } & \multicolumn{2}{|c|}{ Total } \\
\hline & Exports & Imports & Exports & Imports & Exports & Imports & Exports & Imports & Exports & Imports \\
\hline $1992 \ldots \ldots \ldots \ldots \ldots$ & $\$ 42.28$ & $\$ 33.63$ & $\$ 41.34$ & $\$ 32.96$ & $\$ 40.70$ & $\$ 34.43$ & $\$ 41.07$ & $\$ 33.08$ & $\$ 41.34$ & $\$ 33.46$ \\
\hline $1993 \ldots \ldots \ldots \ldots$ & 42.46 & 30.70 & 41.42 & 32.26 & 40.72 & 29.52 & 41.00 & 28.91 & 41.41 & 29.89 \\
\hline 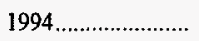 & 41.89 & 28.86 & 40.01 & 28.73 & 38.86 & 30.92 & 39.43 & 31.93 & 39.93 & 30.21 \\
\hline 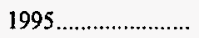 & 39.90 & 32.33 & 39.59 & 36.16 & 40.99 & 33.61 & 40.55 & 34.54 & 40.27 & 34.13 \\
\hline 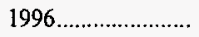 & 41.77 & 33.52 & 40.78 & 32.46 & 40.53 & 33.19 & 40.08 & 34.55 & 40.76 & 33.45 \\
\hline $1997 \ldots \ldots \ldots \ldots \ldots \ldots$ & 41.72 & 33.85 & 40.80 & 35.26 & 39.42 & 33.69 & 40.40 & 34.49 & 40.55 & 34.32 \\
\hline 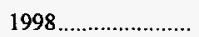 & 41.90 & 33.98 & 38.60 & 31.43 & NA & NA & NA & NA & NA & NA \\
\hline
\end{tabular}

NA Not available.

Notes: Exports: Average price is based on the free alongside ship (f.a.s.) value. Imports: Average price is based on the customs import value. More detailed data included in Table A4. Total may not equal sum of components because of independent rounding.

Sources: Exports: Bureau of the Census, U.S. Department of Commerce, "Monthly Report EM 545;"' and Imports: Bureau of the Census, U.S. Department of Commerce, "Monthly Report IM 145." 
Table 8. U.S. Coal Exports

(Short Tons)

\begin{tabular}{|c|c|c|c|c|c|c|}
\hline \multirow{2}{*}{$\begin{array}{l}\text { Continent and Country } \\
\text { of Destination }\end{array}$} & \multirow{2}{*}{$\begin{array}{c}\text { April - } \\
\text { June } \\
1998\end{array}$} & \multirow{2}{*}{$\begin{array}{c}\text { January - } \\
\text { March } \\
1998\end{array}$} & \multirow{2}{*}{$\begin{array}{l}\text { April - } \\
\text { June } \\
1997\end{array}$} & \multicolumn{3}{|c|}{ Year to date } \\
\hline & & & & 1998 & 1997 & $\begin{array}{l}\text { Percent } \\
\text { Change }\end{array}$ \\
\hline 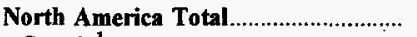 & $6,220,099$ & $2,455,244$ & $4,061,358$ & $8,675,343$ & $5,948,363$ & 45.8 \\
\hline 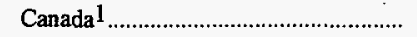 & $5,806,393$ & $1,999,706$ & $3,564,963$ & $7,806,099$ & $5,019,148$ & 55.5 \\
\hline Dominican Republic ................................ & 21,108 & 43,901 & - & 65,009 & - & - \\
\hline 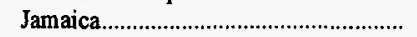 & - & 14,779 & 14,261 & 14,779 & 27,844 & -46.9 \\
\hline 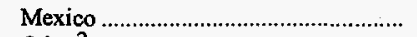 & 388,769 & 393,444 & 482,134 & 782,213 & 895,755 & -12.7 \\
\hline Other 2 & 3,829 & 3,414 & - & 7,243 & 5,616 & 29.0 \\
\hline South America Total & $1,824,955$ & $2,008,237$ & $2,021,661$ & $3,833,192$ & $4,165,531$ & -8.0 \\
\hline 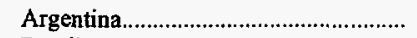 & 88,108 & 89,757 & 15,877 & 177,865 & 176,892 & .6 \\
\hline 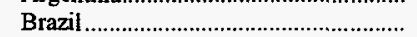 & $1,675,778$ & $1,788,968$ & $1,828,935$ & $3,464,746$ & $3,754,570$ & -7.7 \\
\hline Chile & 434 & 50,279 & 90,518 & 50,713 & 95,999 & -47.2 \\
\hline Other ${ }^{2}$ & 60,635 & 79,233 & 86,331 & 139,868 & 138,070 & 1.3 \\
\hline 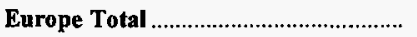 & $8,847,729$ & $9,375,173$ & $10,796,233$ & $18,222,902$ & $21,875,993$ & -16.7 \\
\hline Belgium \& Luxembourg .......................... & 964,236 & 987,540 & 830,745 & $1,951,776$ & $2,212,636$ & -11.8 \\
\hline Bulgaria & 372,706 & 506,231 & 440,807 & 878,937 & 663,504 & 32.5 \\
\hline 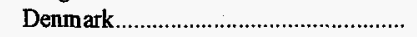 & 119,524 & 154,909 & 109,105 & 274,433 & 224,847 & 22.1 \\
\hline Finland & 202,862 & - & 204,381 & 202,862 & 204,381 & -.7 \\
\hline France & 792,175 & 874,249 & 891,402 & $1,666,424$ & $1,824,906$ & -8.7 \\
\hline Germany, FR & 375,650 & 238,807 & 297,117 & 614,457 & 427,658 & 43.7 \\
\hline Ireland & 134,670 & 491,501 & 118,621 & 626,171 & 382,134 & 63.9 \\
\hline Italy & $1,459,751$ & $1,612,341$ & $1,859,879$ & $3,072,092$ & $3,589,967$ & -14.4 \\
\hline 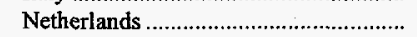 & 929,105 & $1,169,414$ & 929,093 & $2,098,519$ & $2,609,438$ & -19.6 \\
\hline 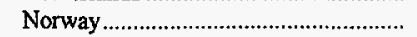 & 13,246 & 25,091 & 37,430 & 38,337 & 54,733 & -30.0 \\
\hline 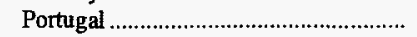 & 205,670 & 147,835 & 366,709 & 353,505 & 569,067 & -37.9 \\
\hline Romania & 533,098 & 350,985 & 726,902 & 884,083 & $1,025,481$ & -13.8 \\
\hline 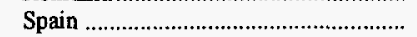 & 806,531 & 876,153 & $1,156,369$ & $1,682,684$ & $2,154,340$ & -21.9 \\
\hline Sweden & 81,004 & 77,572 & 88,255 & 158,576 & 169,729 & -6.6 \\
\hline 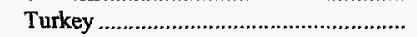 & 427,660 & 609,949 & 657,443 & $1,037,609$ & $1,253,627$ & -17.2 \\
\hline United Kingdom .................................... & $1,415,188$ & $1,237,380$ & $2,067,044$ & $2,652,568$ & $4,477,836$ & -40.8 \\
\hline Other 2 & 14,653 & 15,216 & 14,931 & 29,869 & 31,709 & -5.8 \\
\hline Asia Total & 2,848,527 & $3,700,228$ & $3,222,816$ & $6,548,755$ & $7,178,126$ & -8.8 \\
\hline China (Taiwan) & 406,838 & 274,409 & 384,319 & 681,247 & $1,024,800$ & -33.5 \\
\hline 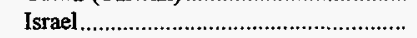 & 151,191 & 182,322 & 45,333 & 333,513 & 331,637 & .6 \\
\hline Japan & $1,602,131$ & $2,492,550$ & $1,826,810$ & $4,094,681$ & $4,187,242$ & -2.2 \\
\hline 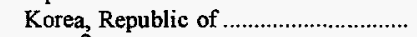 & 667,827 & 720,284 & 964,784 & $1,388,111$ & $1,598,127$ & -13.1 \\
\hline Other ${ }^{2}$ & 20,540 & 30,663 & 1,570 & 51,203 & 36,320 & 41.0 \\
\hline Oceania \& Australia Total .................... & 170 & 3,963 & 609 & 4,133 & 609 & (3) \\
\hline 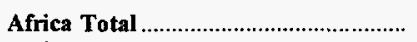 & 758,073 & 750,919 & 500,590 & $1,508,992$ & $1,445,716$ & 4.4 \\
\hline 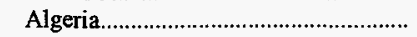 & 55,102 & 114,523 & 55,030 & 169,625 & 110,010 & 54.2 \\
\hline Egypt & 367,253 & $.316,871$ & 255,832 & 684,124 & 635,722 & 7.6 \\
\hline 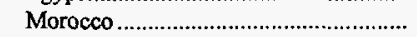 & 68,102 & - & - & 68,102 & 141,980 & -52.0 \\
\hline South Africa, Rep of & 264,095 & 315,309 & 189,400 & 579,404 & 557,676 & 3.9 \\
\hline Other ${ }^{2}$ & 3,521 & 4,216 & 328 & 7,737 & 328 & (3) \\
\hline Total & $20,499,553$ & $18,293,764$ & $20,603,267$ & $38,793,317$ & $40,614,338$ & -4.5 \\
\hline
\end{tabular}

1 Based on the U.S. - Canada Free Trade Agreement; as of January 1990, the U.S. Department of Commerce began reporting statistics on U.S. exports to Canada based on information on imports provided monthly by the Canadian government

2 Includes countries with exports less than or equal to 50,000 short tons in 1997.

3 Changes of 500 percent or more are not shown.

Note: Total may not equal sum of components because of independent rounding.

Source: Bureau of the Census, U.S. Department of Commerce, "Monthly Report EM 545." 
Table 9. Average Price of U.S. Coal Exports (Dollars per Short Ton)

\begin{tabular}{|c|c|c|c|c|c|c|}
\hline \multirow{2}{*}{$\begin{array}{l}\text { Continent and Country } \\
\text { of Destination }\end{array}$} & \multirow{2}{*}{$\begin{array}{l}\text { April - } \\
\text { June } \\
1998\end{array}$} & \multirow{2}{*}{$\begin{array}{c}\text { January - } \\
\text { March } \\
1998\end{array}$} & \multirow{2}{*}{$\begin{array}{c}\text { April - } \\
\text { June } \\
1997\end{array}$} & \multicolumn{3}{|c|}{ Year to date } \\
\hline & & & & 1998 & 1997 & $\begin{array}{l}\text { Percent } \\
\text { Change }\end{array}$ \\
\hline North America Total... & $\$ 28.54$ & $\$ 29.57$ & $\$ 30.46$ & $\$ 28.83$ & $\$ 31,34$ & -8.0 \\
\hline 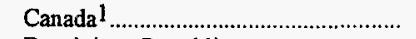 & 28.07 & 27.91 & 29.52 & 28.03 & 30.04 & -6.7 \\
\hline Dominican Republic .................................. & 36.74 & 36.74 & - & 36.74 & - & - \\
\hline 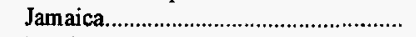 & - & 39.69 & 41.96 & 39.69 & 41.96 & -5.4 \\
\hline Mexico & 34.87 & 36.45 & 36.96 & 35.66 & 38.08 & -6.3 \\
\hline Other $^{2}$ & 40.79 & 41.23 & - & 41.00 & 40.68 & .8 \\
\hline South America Total ............................. & 43.49 & 43.18 & 43.21 & 43.32 & 43.84 & -1.2 \\
\hline 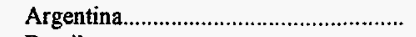 & 43.39 & 46.72 & 40.75 & 45.07 & 47.52 & -5.1 \\
\hline 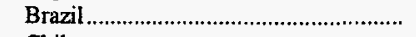 & 43.52 & 43.51 & 43.80 & 43.52 & 44.02 & -1.1 \\
\hline Chile & 40.75 & 28.59 & 33.66 & 28.67 & 34.01 & -15.7 \\
\hline Other $^{2}$ & 42.68 & 40.81 & 40.81 & 41.62 & 40.85 & 1.9 \\
\hline Europe Total & 43.23 & 44.51 & 42.85 & 43.89 & 42.62 & 3.0 \\
\hline Belgium \& Luxembourg ........................ & 44.62 & 46.91 & 47.09 & 45.78 & 46.58 & -1.7 \\
\hline Bulgaria & 42.58 & 46.11 & 47.13 & 44.61 & 46.85 & -4.8 \\
\hline Denmark & 34.02 & 34.02 & 30.80 & 34.02 & 33.55 & 1.4 \\
\hline Finland & 40.46 & - & 41.51 & 40.46 & 41.51 & -2.5 \\
\hline 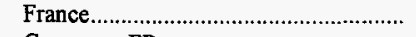 & 46.06 & 46.50 & 45.16 & 46.29 & 45.58 & 1.6 \\
\hline Germany, FR & 33.87 & 41.56 & 45.86 & 36.86 & 44.90 & -17.9 \\
\hline Ireland & 37.42 & 37.70 & 37.42 & 37.64 & 37.77 & -.3 \\
\hline Italy & 46.75 & 46.32 & 44.84 & 46.52 & 45.38 & 2.5 \\
\hline 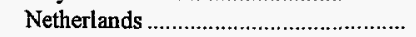 & 44.17 & 47.08 & 45.86 & 45.79 & 44.47 & 3.0 \\
\hline 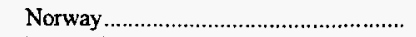 & 57.11 & 57.18 & - & 57.17 & - & - \\
\hline 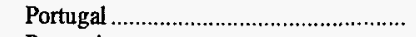 & 37.39 & 40.09 & 35.55 & 38.52 & 36.71 & 4.9 \\
\hline Romania & 42.35 & 43.02 & 48.00 & 42.62 & 47.00 & -9.3 \\
\hline Spain & 41.91 & 44.00 & 34.09 & 43.06 & 35.97 & 19.7 \\
\hline Sweden & 46.80 & 48.45 & 48.34 & 47.61 & 48.32 & -1.5 \\
\hline 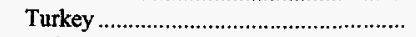 & 46.32 & 44.95 & 46.62 & 45.52 & 46.42 & -1.9 \\
\hline 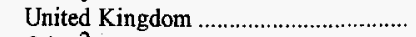 & 41.36 & 40.86 & 39.59 & 41.13 & 37.81 & 8.8 \\
\hline Other 2 & 56.40 & 53.33 & 54.59 & 54.84 & 54.73 & .2 \\
\hline 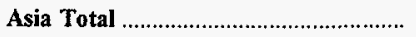 & 39.66 & 40.29 & 40.89 & 40.02 & 40.26 & -.6 \\
\hline 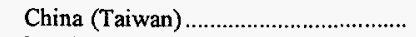 & 34.34 & 36.63 & 34.54 & 35.26 & 36.39 & -3.1 \\
\hline Israel & 31.93 & 36.04 & 37.19 & 34.18 & 37.11 & -7.9 \\
\hline 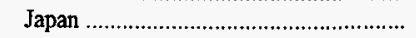 & 39.97 & 39.45 & 40.14 & 39.65 & 39.66 & * \\
\hline 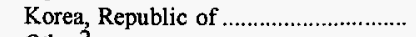 & 43.87 & 45.66 & 45.02 & 44.80 & 45.11 & -.7 \\
\hline Other $^{2}$ & 39.91 & 40.81 & 39.93 & 40.45 & 35.16 & 15.0 \\
\hline Oceania \& Australia Total ..................... & 40.61 & 51.08 & 40.81 & 50.70 & 40.81 & 24.2 \\
\hline Africa Total & 44.77 & 46.22 & 49.90 & 45.48 & 48.82 & -6.8 \\
\hline 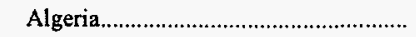 & 41.30 & 45.13 & 46.28 & 43.89 & 47.37 & -7.4 \\
\hline Egypt & 44.53 & 43.94 & 51.48 & 44.26 & 52.85 & -16.3 \\
\hline Morocco & 31.57 & - & - & 31.57 & 30.67 & 2.9 \\
\hline 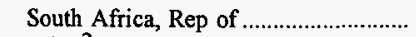 & 49.28 & 49.35 & 48.83 & 49.31 & 49.14 & .4 \\
\hline Other $^{2}$ & 40.81 & 37.21 & 40.76 & 38.85 & 40.76 & -4.7 \\
\hline $\operatorname{Total}^{3}$ & 38.40 & 41.62 & 40.39 & 39.92 & 40.95 & -2.5 \\
\hline 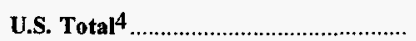 & 38.60 & 41.90 & 40.80 & 40.16 & 41.26 & -2.7 \\
\hline
\end{tabular}

1 Based on the U.S. - Canada Free Trade Agreement; as of January 1990, the U.S. Department of Commerce began reporting statistics on U.S. exports to Canada based on information on imports provided monthly by the Canadian government.

2 Includes countries with exports less than or equal to 50,000 short tons in 1997.

3 The average prices presented in this table, with the exception of U.S. Total, are considered to be representative prices for coal exports and fall within the range of $\$ 20$ to $\$ 60$ per short ton, inclusively.

4 U.S. Total is the average price of all coal exports.

* Rounded to zero

Notes: Total may not equal sum of components because of independent rounding. Average price is based on the free alongside ship (f.a.s.) value.

Source: Bureau of the Census, U.S. Department of Commerce, "Monthly Report EM 545."' 
Table 10. U.S. Steam Coal Exports

(Short Tons)

\begin{tabular}{|c|c|c|c|c|c|c|}
\hline \multirow{2}{*}{$\begin{array}{c}\text { Continent and Country } \\
\text { of Destination }\end{array}$} & \multirow{2}{*}{$\begin{array}{l}\text { April - } \\
\text { June } \\
1998\end{array}$} & \multirow{2}{*}{$\begin{array}{c}\text { January - } \\
\text { March } \\
1998\end{array}$} & \multirow{2}{*}{$\begin{array}{l}\text { April - } \\
\text { June } \\
1997\end{array}$} & \multicolumn{3}{|c|}{ Year to date } \\
\hline & & & & 1998 & 1997 & $\begin{array}{l}\text { Percent } \\
\text { Change }\end{array}$ \\
\hline North America Total................................ & $4,880,569$ & $1,951,953$ & $2,552,607$ & $6,832,522$ & $4,237,965$ & 61.2 \\
\hline 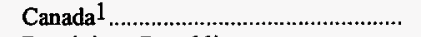 & $4,482,781$ & $1,497,301$ & $2,158,882$ & $5,980,082$ & $3,494,627$ & 71.1 \\
\hline Dominican Republic & 21,108 & 43,901 & - & 65,009 & - & - \\
\hline 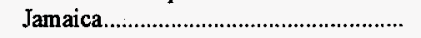 & - & 14,779 & 14,261 & 14,779 & 27,844 & -46.9 \\
\hline Mexico & 372,851 & 392,558 & 379,464 & 765,409 & 709,878 & 7.8 \\
\hline Other ${ }^{2}$ & 3,829 & 3,414 & - & 7,243 & 5,616 & 29.0 \\
\hline 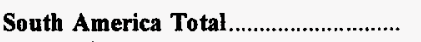 & 45,284 & 145,297 & 228,118 & 190,581 & 351,803 & -45.8 \\
\hline Argentina & 5,394 & 14,985 & 15,877 & 20,379 & 34,219 & -40.4 \\
\hline 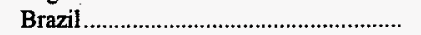 & 9,323 & 800 & 35,392 & 10,123 & 83,515 & -87.9 \\
\hline 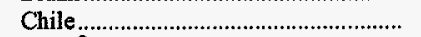 & 434 & 50,279 & 90,518 & 50,713 & 95,999 & -47.2 \\
\hline Other ${ }^{2}$ & 30,133 & 79,233 & 86,331 & 109,366 & 138,070 & -20.8 \\
\hline Europe Total & $1,940,154$ & $2,194,757$ & $3,413,205$ & $4,134,911$ & $7,201,771$ & -42.6 \\
\hline 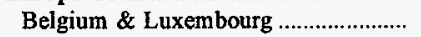 & 127,994 & 126,519 & 80,535 & 254,513 & 375,587 & -32.2 \\
\hline 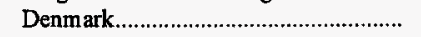 & 119,524 & 154,909 & 109,105 & 274,433 & 224,847 & 22.1 \\
\hline Finland & - & - & 60,361 & - & 60,361 & - \\
\hline France & - & 81,196 & 70,155 & 81,196 & 201,479 & -59.7 \\
\hline Germany, FR & 263,730 & 128,995 & 64,316 & 392,725 & 129,500 & 203.3 \\
\hline Ireland & 134,670 & 491,501 & 118,621 & 626,171 & 261,313 & 139.6 \\
\hline Italy & 283,892 & 390,692 & 657,245 & 674,584 & $1,309,661$ & -48.5 \\
\hline 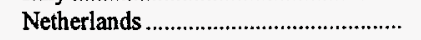 & 102,494 & 50,569 & 79,708 & 153,063 & 428,290 & -64.3 \\
\hline 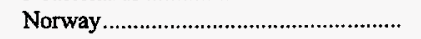 & - & - & 6,638 & - & 6,638 & - \\
\hline 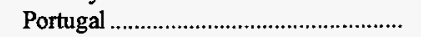 & 132,921 & 81,218 & 366,709 & 214,139 & 496,268 & -56.9 \\
\hline Spain & 248,445 & 167,952 & 677,534 & 416,397 & $1,052,310$ & -60.4 \\
\hline 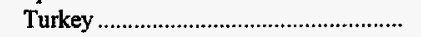 & 295 & 925 & 497 & 1,220 & 1,775 & -31.3 \\
\hline 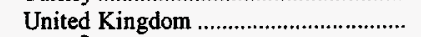 & 526,009 & 518,016 & $1,118,729$ & $1,044,025$ & $2,647,175$ & -60.6 \\
\hline 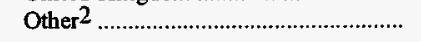 & 180 & 2,265 & 3,052 & 2,445 & 6,567 & -62.8 \\
\hline Asia Total & $1,073,263$ & $1,655,766$ & $1,183,245$ & $2,729,029$ & $3,139,114$ & -13.1 \\
\hline China (Taiwan) & 406,838 & 195,582 & 384,319 & 602,420 & 887,067 & -32.1 \\
\hline Israel & 115,597 & 144,631 & 45,333 & 260,228 & 299,542 & -13.1 \\
\hline 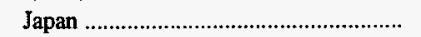 & 372,662 & $1,131,243$ & 515,743 & $1,503,905$ & $1,609,983$ & -6.6 \\
\hline 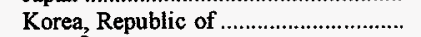 & 157,626 & 153,647 & 236,280 & 311,273 & 318,049 & -2.1 \\
\hline Other ${ }^{2}$ & 20,540 & 30,663 & 1,570 & 51,203 & 24,473 & 109.2 \\
\hline Oceania \& Australia Total ..................... & 170 & 1,132 & 609 & 1,302 & 609 & 113.8 \\
\hline Africa Total & $\mathbf{7 2 , 3 4 7}$ & 4,216 & 578 & 76,563 & 143,309 & -46.6 \\
\hline 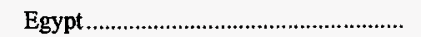 & 724 & - & 250 & 724 & 1,001 & -27.7 \\
\hline Morocco & 68,102 & - & - & 68,102 & 141,980 & -52.0 \\
\hline Other ${ }^{2}$ & 3,521 & 4,216 & 328 & 7,737 & 328 & (3) \\
\hline Total & $8,011,787$ & $5,953,121$ & $7,378,362$ & $13,964,908$ & $15,074,571$ & -7.4 \\
\hline
\end{tabular}

1 Based on the U.S. - Canada Free Trade Agreement; as of January 1990, the U.S. Department of Commerce began reporting statistics on U.S. exports to Canada based on information on imports provided monthly by the Canadian government.

2 Includes countries with exports less than or equal to 50,000 short tons in 1997.

3 Changes of 500 percent or more are not shown.

Notes: Total may not equal sum of components because of independent rounding. Steam coal includes bituminous, subbituminous, lignite, and anthracite.

Source: Bureau of the Census, U.S. Department of Commerce, "Monthly Report EM 545." 
Table 11. Average Price of U.S. Steam Coal Exports (Dollars per Short Ton)

\begin{tabular}{|c|c|c|c|c|c|c|}
\hline \multirow{2}{*}{$\begin{array}{c}\text { Continent and Country } \\
\text { of Destination }\end{array}$} & \multirow{2}{*}{$\begin{array}{l}\text { April - } \\
\text { June } \\
1998\end{array}$} & \multirow{2}{*}{$\begin{array}{c}\text { January - } \\
\text { March } \\
1998\end{array}$} & \multirow{2}{*}{$\begin{array}{l}\text { April - } \\
\text { June } \\
1997\end{array}$} & \multicolumn{3}{|c|}{ Year to date } \\
\hline & & & & 1998 & 1997 & $\begin{array}{l}\text { Percent } \\
\text { Change }\end{array}$ \\
\hline North America Total................................ & $\$ 25.69$ & $\$ 28.57$ & $\$ 27.49$ & $\$ 26.51$ & $\$ 29.37$ & -9.7 \\
\hline 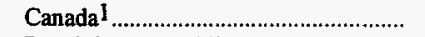 & 24.89 & 26.02 & 26.06 & 25.17 & 27.86 & -9.7 \\
\hline 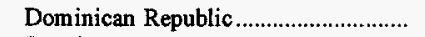 & 36.74 & 36.74 & - & 36.74 & - & - \\
\hline 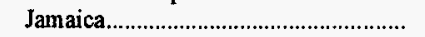 & - & 39.69 & 41.96 & 39.69 & 41.96 & -5.4 \\
\hline 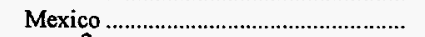 & 34.21 & 36.45 & 34.48 & 35.36 & 35.76 & -1.1 \\
\hline Other 2 & 40.79 & 41.23 & - & 41.00 & 40.68 & .8 \\
\hline South America Total................................. & 40.85 & 36.58 & 37.35 & 37.58 & 39.26 & -4.3 \\
\hline 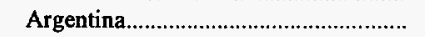 & 40.80 & 40.80 & 40.75 & 40.80 & 40.78 & * \\
\hline 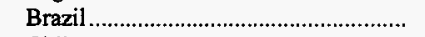 & 40.80 & 40.77 & 37.76 & 40.80 & 42.23 & -3.4 \\
\hline 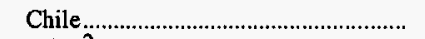 & 40.75 & 28.59 & 33.66 & 28.67 & 34.01 & -15.7 \\
\hline Other ${ }^{2}$ & 40.88 & 40.81 & 40.81 & 40.83 & 40.85 & -.1 \\
\hline Europe Total & 31.71 & 33.93 & 31.95 & 32.91 & 32.25 & 2.1 \\
\hline 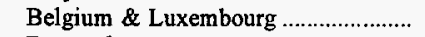 & 35.38 & 35.18 & 37.06 & 35.28 & 37.08 & -4.9 \\
\hline Denmark & 34.02 & 34.02 & 30.80 & 34.02 & 33.55 & 1.4 \\
\hline Finland & - & - & 36.20 & - & 36.20 & - \\
\hline France & - & 36.20 & 37.20 & 36.20 & 35.54 & 1.9 \\
\hline Germany, FR & 28.64 & 35.97 & 37.88 & 31.05 & 37.88 & -18.0 \\
\hline Ireland & 37.42 & 37.70 & 37.42 & 37.64 & 37.94 & -.8 \\
\hline Italy & 38.72 & 38.68 & 38.72 & 38.70 & 40.17 & -3.7 \\
\hline 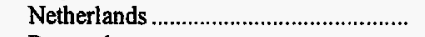 & 28.30 & 24.60 & 31.09 & 27.17 & 32.25 & -15.8 \\
\hline 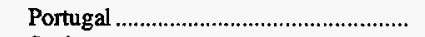 & 33.02 & 36.88 & 35.55 & 34.48 & 35.50 & -2.9 \\
\hline Spain & 22.11 & 21.02 & 22.08 & 21.56 & 21.93 & -1.7 \\
\hline 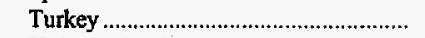 & 40.74 & 40.75 & 40.79 & 40.75 & 40.77 & -.1 \\
\hline United Kingdom & 29.81 & 30.04 & 31.08 & 29.92 & 29.83 & 3 \\
\hline Other $^{2}$ & 40.58 & 34.48 & 34.47 & 34.93 & 34.59 & 1.0 \\
\hline 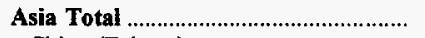 & 33.91 & 35.01 & 34.77 & 34.58 & 35.39 & -2.3 \\
\hline China (Taiwan) & 34.34 & 34.52 & 34.54 & 34.40 & 35.16 & -2.2 \\
\hline Israel & 32.34 & 34.88 & 37.19 & 33.75 & 35.82 & -5.8 \\
\hline Japan & 33.78 & 34.52 & 34.39 & 34.34 & 35.62 & -3.6 \\
\hline 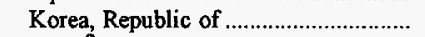 & 33.48 & 38.17 & 35.47 & 35.80 & 35.34 & 1,3 \\
\hline Other $^{2}$ & 39.91 & 40.81 & 39.93 & 40.45 & 24.18 & 67.3 \\
\hline Oceania \& Australia Total...................... & 40.61 & 40.80 & 40.81 & 40.78 & 40.81 & -.1 \\
\hline 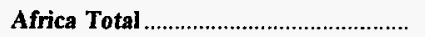 & 32.10 & 37.21 & 40.80 & 32.38 & 30.77 & 5.3 \\
\hline Egypt & 39.88 & - & 40.86 & 39.88 & 40.82 & -2.3 \\
\hline 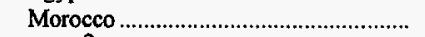 & 31.57 & - & - & 31.57 & 30.67 & 2.9 \\
\hline Other ${ }^{2}$ & 40.81 & 37.21 & 40.76 & 38.85 & 40.76 & -4.7 \\
\hline 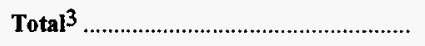 & 28.40 & 32.59 & 31.10 & 30.20 & 32.29 & -6.5 \\
\hline U.S. Total $^{4} \ldots$ & 29.18 & 33.19 & 32.25 & 30.89 & 33.10 & -6.7 \\
\hline
\end{tabular}

1 Based on the U.S. - Canada Free Trade Agreement; as of January 1990, the U.S. Department of Commerce began reporting statistics on U.S. exports to Canada based on information on imports provided monthly by the Canadian government.

2 Includes countries with exports less than or equal to 50,000 short tons in 1997

3 The average prices presented in this table, with the exception of U.S. Total, are considered to be representative prices for coal exports and fall within the range of $\$ 20$ to $\$ 60$ per short ton, inclusively.

4 U.S. Total is the average price of all coal exports.

- Rounded to zero

Notes: Total may not equal sum of components because of independent rounding. Average price is based on the free alongside ship (f.a.s.) value.

Steam coal includes bituminous, subbituminous, lignite, and anthracite.

Source: Bureau of the Census, U.S. Department of Commerce, "Monthly Report EM 545." 
Table 12. U.S. Metallurgical Coal Exports

(Short Tons)

\begin{tabular}{|c|c|c|c|c|c|c|}
\hline \multirow{2}{*}{$\begin{array}{c}\text { Continent and Country } \\
\text { of Destination }\end{array}$} & \multirow{2}{*}{$\begin{array}{l}\text { April - } \\
\text { June } \\
1998\end{array}$} & \multirow{2}{*}{$\begin{array}{c}\text { January - } \\
\text { March } \\
1998\end{array}$} & \multirow{2}{*}{$\begin{array}{l}\text { April - } \\
\text { June } \\
1997\end{array}$} & \multicolumn{3}{|c|}{ Year to date } \\
\hline & & & & 1998 & 1997 & $\begin{array}{l}\text { Percent } \\
\text { Change }\end{array}$ \\
\hline 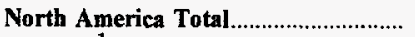 & $1,339,530$ & 503,291 & $1,508,751$ & $1,842,821$ & $1,710,398$ & 7.7 \\
\hline 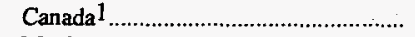 & $1,323,612$ & 502,405 & $1,406,081$ & $1,826,017$ & $1,524,521$ & 19.8 \\
\hline Mexico & 15,918 & 886 & 102,670 & 16,804 & 185,877 & -91.0 \\
\hline South America Total................................ & $1,779,671$ & $1,862,940$ & $1,793,543$ & $3,642,611$ & $3,813,728$ & -4.5 \\
\hline 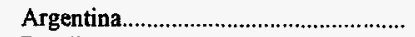 & 82,714 & 74,772 & - & 157,486 & 142,673 & 10.4 \\
\hline 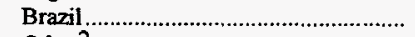 & $1,666,455$ & $1,788,168$ & $1,793,543$ & $3,454,623$ & $3,671,055$ & -5.9 \\
\hline Other ${ }^{2}$ & 30,502 & - & - & 30,502 & - & - \\
\hline 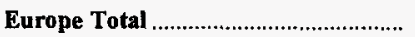 & $6,907,575$ & $7,180,416$ & $7,383,028$ & $14,087,991$ & $14,674,222$ & -4.0 \\
\hline 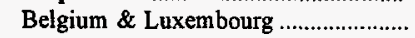 & 836,242 & 861,021 & 750,210 & $1,697,263$ & $1,837,049$ & -7.6 \\
\hline 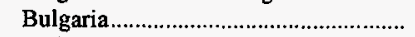 & 372,706 & 506,231 & 440,807 & 878,937 & 663,504 & 32.5 \\
\hline Finland & 202,862 & - & 144,020 & 202,862 & 144,020 & 40.9 \\
\hline France & 792,175 & 793,053 & 821,247 & $1,585,228$ & $1,623,427$ & -2.4 \\
\hline 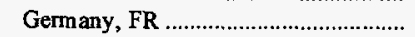 & 111,920 & 109,812 & 232,801 & 221,732 & 298,158 & -25.6 \\
\hline Ireland & - & - & - & - & 120,821 & 20 \\
\hline Italy & $1,175,859$ & $1,221,649$ & $1,202,634$ & $2,397,508$ & $2,280,306$ & 5.1 \\
\hline 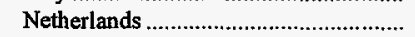 & 826,611 & $1,118,845$ & 849,385 & $1,945,456$ & $2,181,148$ & -10.8 \\
\hline 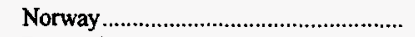 & 13,246 & 25,091 & 30,792 & 38,337 & 48,095 & -20.3 \\
\hline 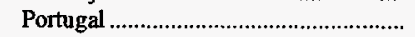 & 72,749 & 66,617 & - & 139,366 & 72,799 & 91.4 \\
\hline Romania & 533,098 & 350,985 & 726,902 & 884,083 & $1,025,481$ & -13.8 \\
\hline Spain & 558,086 & 708,201 & 478,835 & $1,266,287$ & $1,102,030$ & 14.9 \\
\hline Sweden & 81,004 & 77,572 & 88,255 & 158,576 & 169,729 & -6.6 \\
\hline Turkey & 427,365 & 609,024 & 656,946 & $1,036,389$ & $1,251,852$ & -17.2 \\
\hline 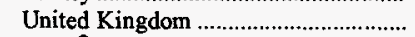 & 889,179 & 719,364 & 948,315 & $1,608,543$ & $1,830,661$ & -12.1 \\
\hline Other ${ }^{2}$ & 14,473 & 12,951 & 11,879 & 27,424 & 25,142 & 9.1 \\
\hline Asia Total & $1,775,264$ & $2,044,462$ & $2,039,571$ & $3,819,726$ & $4,039,012$ & -5.4 \\
\hline China (Taiwan) & - & 78,827 & - & 78,827 & 137,733 & -42.8 \\
\hline 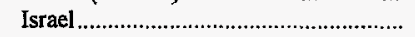 & 35,594 & 37,691 & - & 73,285 & 32,095 & 128.3 \\
\hline Japan & $1,229,469$ & $1,361,307$ & $1,311,067$ & $2,590,776$ & $2,577,259$ & 5 \\
\hline Korea, Republic of & 510,201 & 566,637 & 728,504 & $1,076,838$ & $1,280,078$ & -15.9 \\
\hline Other ${ }^{2}$ & - & - & - & - & 11,847 & - \\
\hline Oceania \& Australia Total ................... & - & 2,831 & - & 2,831 & - & - \\
\hline Africa Total & 685,726 & 746,703 & 500,012 & $1,432,429$ & $1,302,407$ & 10.0 \\
\hline Algeria & 55,102 & 114,523 & 55,030 & 169,625 & 110,010 & 54.2 \\
\hline 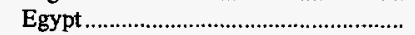 & 366,529 & 316,871 & 255,582 & 683,400 & 634,721 & 7.7 \\
\hline 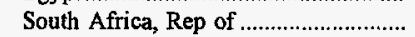 & 264,095 & 315,309 & 189,400 & 579,404 & 557,676 & 3.9 \\
\hline Total & $12,487,766$ & $12,340,643$ & $13,224,905$ & $24,828,409$ & $25,539,767$ & -2.8 \\
\hline
\end{tabular}

1 Based on the U.S. - Canada Free Trade Agreement; as of January 1990, the U.S. Department of Commerce began reporting statistics on U.S. exports to Canada based on information on imports provided monthly by the Canadian government.

2 Includes countries with exports less than or equal to 50,000 short tons in 1997.

Note: Total may not equal sum of components because of independent rounding.

Source: Bureau of the Census, U,S. Department of Commerce, "Monthly Report EM 545." 
Table 13. Average Price of U.S. Metallurgical Coal Exports (Dollars per Short Ton)

\begin{tabular}{|c|c|c|c|c|c|c|}
\hline \multirow{2}{*}{$\begin{array}{l}\text { Continent and Country } \\
\text { of Destination }\end{array}$} & \multirow{2}{*}{$\begin{array}{l}\text { April - } \\
\text { June } \\
1998\end{array}$} & \multirow{2}{*}{$\begin{array}{c}\text { January - } \\
\text { March } \\
1998\end{array}$} & \multirow{2}{*}{$\begin{array}{l}\text { April - } \\
\text { June } \\
1997\end{array}$} & \multicolumn{3}{|c|}{ Year to date } \\
\hline & & & & 1998 & 1997 & $\begin{array}{l}\text { Percent } \\
\text { Change }\end{array}$ \\
\hline North America Total.................................. & $\$ 38.63$ & $\mathbf{\$ 3 3 . 2 9}$ & $\$ 35.19$ & $\$ 37.18$ & $\$ 35.98$ & 3.3 \\
\hline 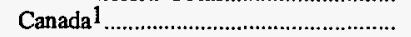 & 38.49 & 33.29 & 34.42 & 37.06 & 34.67 & 6.9 \\
\hline Mexico & 50.29 & - & 47.35 & 50.29 & 47.47 & 6.0 \\
\hline South America TotaI............................... & 43.55 & 43.69 & 43.92 & 43.62 & 44.25 & -1.4 \\
\hline 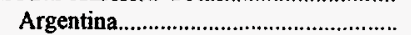 & 43.54 & 47.90 & - & 45.61 & 49.12 & -7.1 \\
\hline 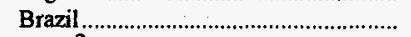 & 43.53 & 43.51 & 43.92 & 43.52 & 44.06 & -1.2 \\
\hline $\mathrm{Other}^{2}$ & 44.45 & - & - & 44.45 & - & - \\
\hline 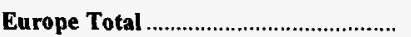 & 46.31 & 47.73 & 47.90 & 47.04 & 47.72 & -1.4 \\
\hline 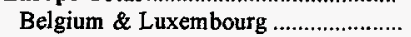 & 46.04 & 48.63 & 48.17 & 47.35 & 48.53 & -2.4 \\
\hline 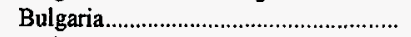 & 42.58 & 46.11 & 47.13 & 44.61 & 46.85 & -4.8 \\
\hline Finland & 40.46 & - & 43.73 & 40.46 & 43.73 & -7.5 \\
\hline 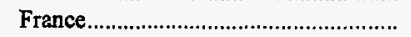 & 46.06 & 47.55 & 45.84 & 46.80 & 46.82 & * \\
\hline 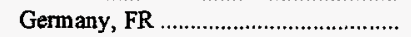 & 46.21 & 48.13 & 48.07 & 47.16 & 47.95 & -1.6 \\
\hline Ireland & - & - & - & - & 37.42 & - \\
\hline Italy & 48.69 & 48.76 & 48.18 & 48.72 & 48.38 & .7 \\
\hline 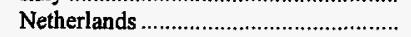 & 46.00 & 47.92 & 47.24 & 47.11 & 46.87 & .5 \\
\hline 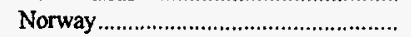 & 57.11 & 57.18 & - & 57.17 & - & - \\
\hline 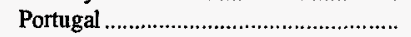 & 45.36 & 44.00 & - & 44.71 & 44.91 & -.4 \\
\hline 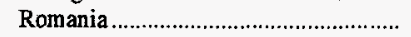 & 42.35 & 43.02 & 48.00 & 42.62 & 47.00 & -9.3 \\
\hline Spain & 47.67 & 49.43 & 51.07 & 48.66 & 49.37 & -1.4 \\
\hline Sweden & 46.80 & 48.45 & 48.34 & 47.61 & 48.32 & -1.5 \\
\hline Turkey & 46.33 & 44.96 & 46.63 & 45.52 & 46.42 & -1.9 \\
\hline 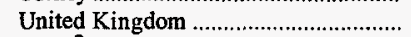 & 48.20 & 48.66 & 49.64 & 48.40 & 49.35 & -1.9 \\
\hline Other ${ }^{2}$ & 56.60 & 56.60 & 59.76 & 56.60 & 59.83 & -5.4 \\
\hline 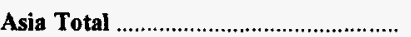 & 43.13 & 44.57 & 44.44 & 43.90 & 44.05 & -.3 \\
\hline China (Taiwan) & - & 41.86 & - & 41.86 & 44.30 & -5.5 \\
\hline 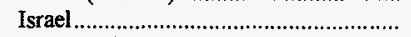 & 30.58 & 40.51 & - & 35.69 & 49.08 & -27.3 \\
\hline 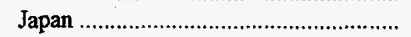 & 41.85 & 43.55 & 42.41 & 42.74 & 42.18 & 1.3 \\
\hline 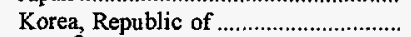 & 47,08 & 47.69 & 48.13 & 47.40 & 47.54 & -.3 \\
\hline Other ${ }^{2}$ & - & - & - & - & 57.51 & - \\
\hline Oceania \& Australia Total................... & - & 55.11 & - & 55.11 & - & - \\
\hline Africa Total & 46.10 & 46.28 & 49.91 & 46.19 & 50.81 & -9.1 \\
\hline 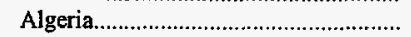 & 41.30 & 45.13 & 46.28 & 43.89 & 47.37 & -7.4 \\
\hline Egypt & 44.54 & 43.94 & 51.49 & 44.26 & 52.87 & -16.3 \\
\hline 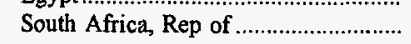 & 49.28 & 49.35 & 48.83 & 49.31 & 49.14 & .4 \\
\hline Total $^{3}$ & 44.63 & 45.92 & 45.46 & 45.27 & 45.99 & -1.6 \\
\hline 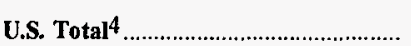 & 44.65 & 46.09 & 45.58 & $\mathbf{4 5 . 3 7}$ & 46.07 & -1.5 \\
\hline
\end{tabular}

1 Based on the U.S. - Canada Free Trade Agreement; as of January 1990, the U.S. Department of Commerce began reporting statistics on U.S. exports to Canada based on information on imports provided monthly by the Canadian government.

2 Includes countries with exports less than or equal to 50,000 short tons in 1997.

3 The average prices presented in this table, with the exception of U.S. Total, are considered to be representative prices for coal exports and fall within the range of $\$ 20$ to $\$ 60$ per short ton, inclusively.

4 U.S. Total is the average price of all coal exports.

* Rounded to zero

Notes: Total may not equal sum of components because of independent rounding. Average price is based on the free alongside ship (f.a.s.) value.

Source: Bureau of the Census, U.S. Department of Commerce, "Monthly Report EM 545." 
Table 14. Coal Exports by Customs District

(Short Tons)

\begin{tabular}{|c|c|c|c|c|c|c|}
\hline \multirow[b]{2}{*}{ Customs District } & \multirow{2}{*}{$\begin{array}{c}\text { April - } \\
\text { June } \\
1998\end{array}$} & \multirow{2}{*}{$\begin{array}{c}\text { January - } \\
\text { March } \\
1998\end{array}$} & \multirow{2}{*}{$\begin{array}{l}\text { April - } \\
\text { June } \\
1997\end{array}$} & \multicolumn{3}{|c|}{ Year to date } \\
\hline & & & & 1998 & 1997 & $\begin{array}{l}\text { Percent } \\
\text { Change }\end{array}$ \\
\hline 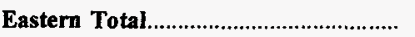 & $12,799,537$ & $12,999,241$ & $13,210,949$ & $25,798,778$ & $26,718,543$ & -3.4 \\
\hline Baltimore, MD & $1,511,366$ & $2,142,712$ & $1,740,467$ & $3,654,078$ & $3,832,478$ & -4.7 \\
\hline Portland, ME & 702 & 101,851 & 152 & 102,553 & 238 & (1) \\
\hline Buffalo, NY & $1,500,525$ & 480,170 & $1,036,406$ & $1,980,695$ & $1,366,982$ & 44.9 \\
\hline 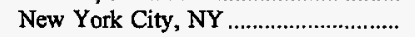 & 5,965 & 2,547 & 335 & 8,512 & 1,223 & (1) \\
\hline 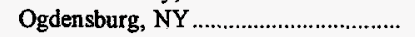 & 47,791 & 6,862 & 38,921 & 54,653 & 43,312 & 26.2 \\
\hline Philadelphia, PA ................................... & 58,377 & 44,670 & 95,114 & 103,047 & 131,670 & -21.7 \\
\hline 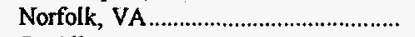 & $9,674,811$ & $10,220,429$ & $10,299,324$ & $19,895,240$ & $21,341,623$ & -6.8 \\
\hline St. Albans, VT & - & - & 230 & - & 1,017 & - \\
\hline 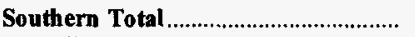 & $2,852,580$ & $2,870,711$ & $3,998,617$ & $5,723,291$ & $8,250,154$ & -30.6 \\
\hline Mobile, AL & $1,343,999$ & $1,150,602$ & $1,535,799$ & $2,494,601$ & $2,911,062$ & -14.3 \\
\hline Savannah, GA & 293 & 1,110 & - & 1,403 & 27,655 & -94.9 \\
\hline 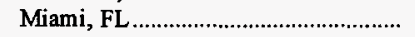 & 184 & 892 & 161 & 1,076 & 161 & (3) \\
\hline 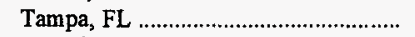 & - & - & 45 & - & 45 & - \\
\hline New Orleans, LA & $1,055,103$ & $1,098,944$ & $1,841,290$ & $2,154,047$ & $4,169,117$ & -48.3 \\
\hline Wilmington, NC & - & - & 20 & - & 20 & - \\
\hline San Juan, PR & - & 39,742 & - & 39,742 & 23 & ( \\
\hline Charleston, SC & 68 & 23 & 42,805 & 91 & 125,375 & -99.9 \\
\hline Houston-Galveston, TX......................... & 64,164 & 188,557 & 186,247 & 252,721 & 294,032 & -14.0 \\
\hline Laredo, TX & 388,769 & 390,841 & 392,250 & 779,610 & 722,664 & 7.9 \\
\hline 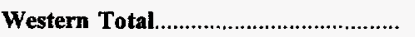 & 717,975 & $1,215,035$ & 997,307 & $1,933,010$ & $2,339,136$ & -17.4 \\
\hline Anchorage, AK & 187,230 & 77,581 & 236,260 & 264,811 & 315,000 & -15.9 \\
\hline 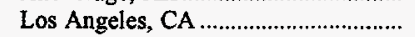 & 530,607 & $1,131,720$ & 674,093 & $1,662,327$ & $1,915,206$ & -13.2 \\
\hline 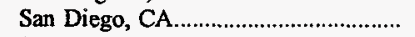 & - & 2,603 & 241 & 2,603 & 241 & (l) \\
\hline 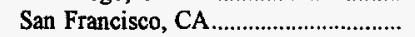 & - & 2,831 & 73,587 & 2,831 & 73,587 & -96.2 \\
\hline 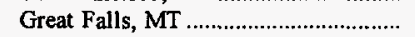 & - & 71 & 86 & 71 & 142 & -50.0 \\
\hline 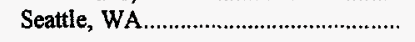 & 138 & 229 & 13,040 & 367 & 34,960 & -99.0 \\
\hline 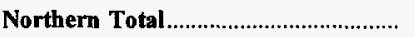 & $4,123,578$ & $1,202,429$ & $2,391,361$ & $5,326,007$ & $3,294,773$ & 61.7 \\
\hline Detroit, MI & 398,910 & 135,655 & 761,320 & 534,565 & $1,487,393$ & -64.1 \\
\hline Duluth, MN & 90,655 & - & 184 & 90,655 & 184 & (I) \\
\hline Pembina, ND & 317 & 577 & 169 & 894 & 198 & 351.5 \\
\hline Cleveland, $\mathrm{OH}$ & $3,633,651$ & $1,066,197$ & $1,629,688$ & $4,699,848$ & $1,806,998$ & 160.1 \\
\hline 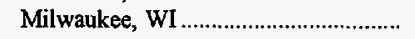 & 45 & - & - & 45 & - & - \\
\hline Other Ports & 5,883 & 6,348 & 5,033 & 12,231 & 11,732 & 4.3 \\
\hline Total & $20,499,553$ & $18,293,764$ & $20,603,267$ & $38,793,317$ & $40,614,338$ & -4.5 \\
\hline
\end{tabular}

1 Changes of 500 percent or more are not shown.

Note: Total may not equal sum of components because of independent rounding.

Source: Bureau of the Census, U.S. Department of Commerce, "Monthly Report EM 545." 
Table 15. U.S. Coke Exports

(Short Tons)

\begin{tabular}{|c|c|c|c|c|c|c|}
\hline \multirow{2}{*}{$\begin{array}{l}\text { Continent and Country } \\
\text { of Destination }\end{array}$} & \multirow{2}{*}{$\begin{array}{c}\text { April - } \\
\text { June } \\
1998\end{array}$} & \multirow{2}{*}{$\begin{array}{c}\text { January - } \\
\text { March } \\
1998\end{array}$} & \multirow{2}{*}{$\begin{array}{l}\text { April - } \\
\text { June } \\
1997\end{array}$} & \multicolumn{3}{|c|}{ Year to date } \\
\hline & & & & 1998 & 1997 & $\begin{array}{l}\text { Percent } \\
\text { Change }\end{array}$ \\
\hline North America Total.............................. & 208,216 & $\mathbf{7 4 , 7 9 1}$ & 232,234 & 283,007 & 283,812 & -0.3 \\
\hline 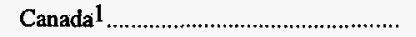 & 117,286 & 2,156 & 185,036 & 119,442 & 187,369 & -36.3 \\
\hline Mexico & 89,810 & 72,139 & 46,935 & 161,949 & 95,585 & 69.4 \\
\hline Other $^{2}$ & 1,120 & 496 & 263 & 1,616 & 858 & 88.3 \\
\hline South America Total ................................ & - & - & - & - & 11,563 & - \\
\hline 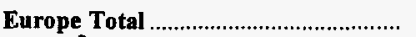 & - & 4,704 & 50,241 & 4,704 & 50,241 & -90.6 \\
\hline Other ${ }^{2}$ & - & 4,704 & 50,241 & 4,704 & 50,241 & -90.6 \\
\hline 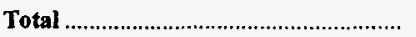 & 208,216 & 79,495 & 282,475 & 287,711 & 345,616 & -16.8 \\
\hline
\end{tabular}

1 Based on the U.S. - Canada Free Trade Agreement; as of January 1990, the U.S. Department of Commerce began reporting statistics on U.S. exports

to Canada based on information on imports provided monthly by the Canadian government.

2 Includes countries with exports less than or equal to 50,000 short tons in 1997 .

Note: Total may not equal sum of components because of independent rounding.

Source: Bureau of the Census, U.S. Department of Commerce, "Monthly Report EM 545."

Table 16. U.S. Coal Imports

(Short Tons)

\begin{tabular}{|c|c|c|c|c|c|c|}
\hline \multirow{2}{*}{$\begin{array}{l}\text { Continent and Country } \\
\text { of Origin }\end{array}$} & \multirow{2}{*}{$\begin{array}{l}\text { April - } \\
\text { June } \\
1998\end{array}$} & \multirow{2}{*}{$\begin{array}{c}\text { January - } \\
\text { March } \\
1998\end{array}$} & \multirow{2}{*}{$\begin{array}{l}\text { April - } \\
\text { June } \\
1997\end{array}$} & \multicolumn{3}{|c|}{ Year to date } \\
\hline & & & & 1998 & 1997 & $\begin{array}{l}\text { Percent } \\
\text { Change }\end{array}$ \\
\hline North America Total................................ & 298,448 & 131,459 & 317,562 & 429,907 & 498,429 & -13.7 \\
\hline 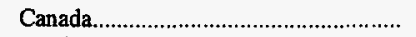 & 297,754 & 131,459 & 317,562 & 429,213 & 498,076 & -13.8 \\
\hline Mexico & 694 & - & - & 694 & 353 & 96.6 \\
\hline South America Total................................ & $1,489,803$ & $1,425,452$ & $1,164,401$ & $2,915,255$ & $1,803,962$ & 61.6 \\
\hline Argentina. & - & - & 13 & - & 13 & - \\
\hline Colombia & 844,210 & 658,637 & 874,727 & $1,502,847$ & $1,367,214$ & 9.9 \\
\hline 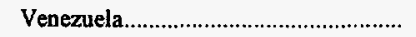 & 645,593 & 766,815 & 289,661 & $1,412,408$ & 436,735 & 223.4 \\
\hline Europe Total & 437 & 4,098 & 8,094 & 4,535 & 15,921 & -71.5 \\
\hline 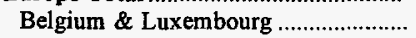 & - & 1,847 & 1,241 & 1,847 & 2,479 & -25.5 \\
\hline Germany, FR & - & - & 1 & - & 1 & - \\
\hline Italy & - & 25 & - & 25 & - & - \\
\hline 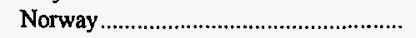 & - & - & 6,852 & - & 13,441 & - \\
\hline 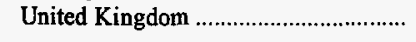 & 437 & 2,226 & - & 2,663 & - & - \\
\hline Asia Total & 403,818 & 246,462 & 187,219 & 650,280 & 595,198 & 9.3 \\
\hline 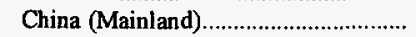 & 525 & 815 & 303 & 1,340 & 452 & 196.5 \\
\hline Indonesia & 403,293 & 245,647 & 186,916 & 648,940 & 582,622 & 11.4 \\
\hline Vietnam & - & - & - & - & 12,124 & - \\
\hline Oceania \& Australia Total ................... & - & $\mathbf{3 1 , 5 2 3}$ & 30,307 & 31,523 & 125,463 & -74.9 \\
\hline 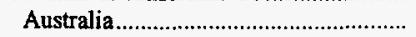 & - & 31,523 & 30,307 & 31,523 & 84,458 & -62.7 \\
\hline 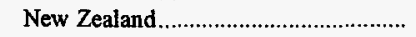 & - & - & - & - & 41,005 & - \\
\hline Total & $2,192,506$ & $1,838,994$ & $1,707,583$ & $4,031,500$ & $3,038,973$ & 32.7 \\
\hline
\end{tabular}

Notes: Total may not equal sum of components because of independent rounding. Coal imports include coal to Puerto Rico and the Virgin Islands. Source: Bureau of the Census, U.S. Department of Commerce, "Monthly Report IM 145." 
Table 17. Average Price of U.S. Coal Imports

(Dollars per Short Ton)

\begin{tabular}{|c|c|c|c|c|c|c|}
\hline \multirow{2}{*}{$\begin{array}{l}\text { Continent and Country } \\
\text { of Origin }\end{array}$} & \multirow{2}{*}{$\begin{array}{l}\text { April - } \\
\text { June } \\
1998\end{array}$} & \multirow{2}{*}{$\begin{array}{l}\text { January - } \\
\text { March } \\
1998\end{array}$} & \multirow{2}{*}{$\begin{array}{l}\text { April - } \\
\text { June } \\
1997\end{array}$} & \multicolumn{3}{|c|}{ Year to date } \\
\hline & & & & 1998 & 1997 & $\begin{array}{l}\text { Percent } \\
\text { Change }\end{array}$ \\
\hline 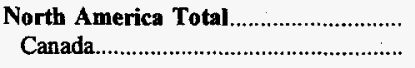 & $\begin{array}{r}\$ 33.83 \\
33.83\end{array}$ & $\begin{array}{r}\$ 33.66 \\
33.66\end{array}$ & $\begin{array}{r}\$ 39.87 \\
39.87\end{array}$ & $\begin{array}{r}\$ 33.78 \\
33.78\end{array}$ & $\begin{array}{r}\$ 35.31 \\
35.31\end{array}$ & $\begin{array}{l}-4.3 \\
-4.3\end{array}$ \\
\hline 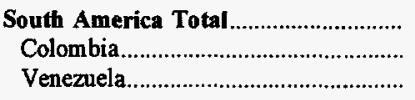 & $\begin{array}{l}30.01 \\
30.42 \\
29.48\end{array}$ & $\begin{array}{l}32.66 \\
33.01 \\
32.36\end{array}$ & $\begin{array}{l}32.79 \\
32.64 \\
33.23\end{array}$ & $\begin{array}{l}31.31 \\
31.55 \\
31.05\end{array}$ & $\begin{array}{l}32.47 \\
32.40 \\
32.69\end{array}$ & $\begin{array}{l}-3.6 \\
-2.6 \\
-5.0\end{array}$ \\
\hline 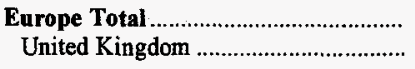 & - & $\begin{array}{l}42.03 \\
42.03\end{array}$ & - & $\begin{array}{l}42.03 \\
42.03\end{array}$ & - & - \\
\hline Asia Total & $\begin{array}{l}32.87 \\
32.87\end{array}$ & $\begin{array}{l}38.77 \\
38.77\end{array}$ & $\begin{array}{l}36.48 \\
36.48\end{array}$ & $\begin{array}{l}35.10 \\
35.10\end{array}$ & $\begin{array}{l}33.32 \\
33.32\end{array}$ & $\begin{array}{l}5.4 \\
5.4\end{array}$ \\
\hline 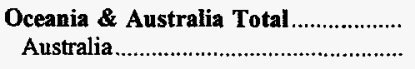 & - & $\begin{array}{l}31.35 \\
31.35\end{array}$ & $\begin{array}{l}34.95 \\
34.95\end{array}$ & $\begin{array}{l}31.35 \\
31.35\end{array}$ & $\begin{array}{l}34.21 \\
34.21\end{array}$ & $\begin{array}{l}-8.4 \\
-8.4\end{array}$ \\
\hline Totall & 31.04 & 33.54 & 34.48 & 32.18 & 33.13 & -2.9 \\
\hline U.S. Total ${ }^{2}$ & 31.43 & 33.98 & 35.26 & 32.59 & 34.64 & -5.9 \\
\hline
\end{tabular}

1 The average prices presented in this table, with the exception of U.S. Total, are considered to be representative prices for coal imports and fall within the range of $\$ 20$ to $\$ 55$ per short ton, inclusively.

2 U.S. Total is the average price of all coal imports.

Notes: Total may not equal sum of components because of independent rounding. Average price is based on the customs import value. Coal imports include coal to Puerto Rico and the Virgin Islands.

Source: Bureau of the Census, U.S. Department of Commerce, "Monthiy Report IM 145." 
Table 18. Coal Imports by Customs District (Short Tons)

\begin{tabular}{|c|c|c|c|c|c|c|}
\hline \multirow[b]{2}{*}{ Customs District } & \multirow{2}{*}{$\begin{array}{c}\text { April - } \\
\text { June } \\
1998\end{array}$} & \multirow{2}{*}{$\begin{array}{c}\text { January - } \\
\text { March } \\
1998\end{array}$} & \multirow{2}{*}{$\begin{array}{c}\text { April - } \\
\text { June } \\
1997\end{array}$} & \multicolumn{3}{|c|}{ Year to date } \\
\hline & & & & 1998 & 1997 & $\begin{array}{l}\text { Percent } \\
\text { Change }\end{array}$ \\
\hline 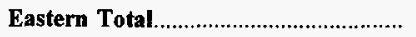 & 449,097 & 724,193 & 600,535 & $1,173,290$ & 984,731 & 19.1 \\
\hline 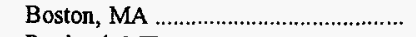 & 228,234 & 465,919 & 397,303 & 694,153 & 624,182 & 11.2 \\
\hline 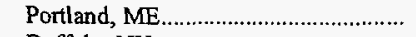 & 116,021 & 34,681 & 69,258 & 150,702 & 155,968 & -3.4 \\
\hline 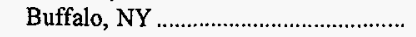 & - & 1,847 & 1,241 & 1,847 & 2,479 & -25.5 \\
\hline 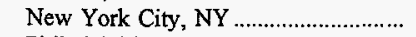 & 104,842 & 157,392 & 105,507 & 262,234 & 174,876 & 50.0 \\
\hline Philadelphia, PA & - & 64,354 & 27,226 & 64,354 & 27,226 & 136.4 \\
\hline Southern Total & $1,258,316$ & 779,193 & 633,349 & $2,037,509$ & $1,162,243$ & 75.3 \\
\hline 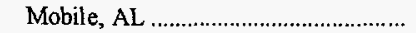 & 356,851 & 97,266 & 34,975 & 454,117 & 98,865 & 359.3 \\
\hline Savannah, GA & 63,824 & 76,061 & 43,120 & 139,885 & 68,920 & 103.0 \\
\hline Miami, FL & 45,093 & 45,288 & 13 & 90,381 & 13 & (b) \\
\hline Tampa, FL & 340,463 & 343,005 & 425,655 & 683,468 & 564,554 & 21.1 \\
\hline New Orleans, LA & 223,471 & 153,601 & 68,242 & 377,072 & 328,056 & 14.9 \\
\hline San Juan, PR & 54,186 & 57,904 & 61,344 & 112,090 & 89,358 & 25.4 \\
\hline Houston-Galveston, TX & 117,290 & 6,068 & - & 123,358 & 12,124 & $($ ) \\
\hline 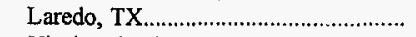 & 694 & - & - & 694 & 353 & 96.6 \\
\hline 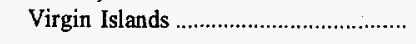 & 56,444 & - & - & 56,444 & - & - \\
\hline 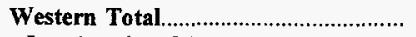 & 212,168 & 230,465 & 171,839 & 442,633 & 437,052 & 1.3 \\
\hline Los Angeles, CA & - & - & - & - & 149 & - \\
\hline 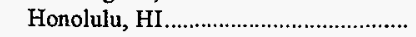 & 179,822 & 203,334 & 148,982 & 383,156 & 380,030 & .8 \\
\hline 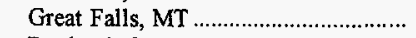 & - & - & 281 & - & 281 & - \\
\hline Portland, OR & 6,992 & - & 6,852 & 6,992 & 13,441 & -48.0 \\
\hline Seattle, WA & 25,354 & 27,131 & 15,724 & 52,485 & 43,151 & 21.6 \\
\hline Northern Total & 272,925 & 105,143 & 301,860 & 378,068 & 454,947 & -16.9 \\
\hline Chicago, IL & 33,746 & - & 92,757 & 33,746 & 92,757 & -63.6 \\
\hline 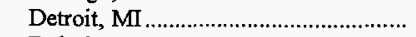 & 63,314 & 34,814 & 125,882 & 98,128 & 131,353 & -25.3 \\
\hline 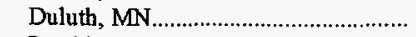 & - & - & - & - & 416 & - \\
\hline Pembina, ND & 175,340 & 69,514 & 82,918 & 244,854 & 230,118 & 6.4 \\
\hline 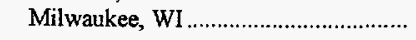 & 525 & 815 & 303 & 1,340 & 303 & 342.2 \\
\hline Total & $2,192,506$ & $1,838,994$ & $1,707,583$ & $4,031,500$ & $3,038,973$ & 32.7 \\
\hline
\end{tabular}

1 Changes of 500 percent or more are not shown.

Note: Total may not equal sum of components because of independent rounding.

Source: Bureau of the Census, U.S. Department of Commerce, "Monthly Report IM 145."

Table 19. U.S. Coke Imports

(Short Tons)

\begin{tabular}{|c|c|c|c|c|c|c|}
\hline \multirow{2}{*}{$\begin{array}{c}\text { Continent and Country } \\
\text { of Origin }\end{array}$} & \multirow{2}{*}{$\begin{array}{l}\text { April - } \\
\text { June } \\
1998\end{array}$} & \multirow{2}{*}{$\begin{array}{c}\text { January - } \\
\text { March } \\
1998\end{array}$} & \multirow{2}{*}{$\begin{array}{l}\text { April - } \\
\text { June } \\
1997\end{array}$} & \multicolumn{3}{|c|}{ Year to date } \\
\hline & & & & 1998 & 1997 & $\begin{array}{l}\text { Percent } \\
\text { Change }\end{array}$ \\
\hline North America Total................................. & 7,414 & 4,127 & 9,306 & 11,541 & 14,986 & -23.0 \\
\hline 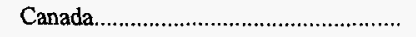 & 7,414 & 4,127 & 9,306 & 11,541 & 14,986 & -23.0 \\
\hline Asia Total & 364,844 & 380,413 & 401,746 & 745,257 & 666,665 & 11.8 \\
\hline 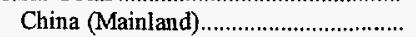 & 140,332 & 198,908 & 142,013 & 339,240 & 188,698 & 79.8 \\
\hline 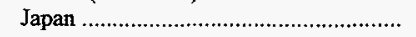 & 224,512 & 181,505 & 259,733 & 406,017 & 477,967 & -15.1 \\
\hline Total & 372,258 & 384,540 & 411,052 & 756,798 & 681,651 & 11.0 \\
\hline
\end{tabular}

Note: Total may not equal sum of components because of independent rounding.

Source: Bureau of the Census, U.S. Department of Commerce, "Monthly Report IM 145." 


\section{Receipts}


Table 20. U.S. Coal Receipts by End-Use Sector, 1992-1998 (Thousand Short Tons)

\begin{tabular}{|c|c|c|c|c|c|}
\hline $\begin{array}{l}\text { Year and } \\
\text { Quarter }\end{array}$ & $\begin{array}{l}\text { Electric } \\
\text { Utilities }\end{array}$ & $\begin{array}{c}\text { Coke } \\
\text { Plants }\end{array}$ & $\begin{array}{c}\text { Other } \\
\text { Industrial }\end{array}$ & $\begin{array}{c}\text { Residential } \\
\text { and Commercial }\end{array}$ & Total \\
\hline 1992 January - March ...................... & 190,139 & 8,410 & 17,902 & 1,843 & 218,294 \\
\hline 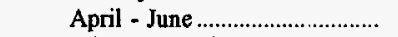 & 187,772 & 7,915 & 17,873 & 1,149 & 214,708 \\
\hline 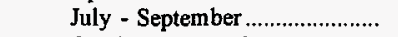 & 201,143 & 7,592 & 18,503 & 1,236 & 228,473 \\
\hline October - December...................... & 196,909 & 8,110 & 19,625 & 1,925 & 226,569 \\
\hline 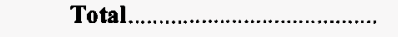 & 775,963 & 32,027 & 73,903 & 6,153 & 888,046 \\
\hline 1993 January - March ........................... & 188,401 & 7,951 & 18,095 & 1,817 & 216,264 \\
\hline April - June & 190,085 & 8,067 & 18,062 & 1,354 & 217,568 \\
\hline 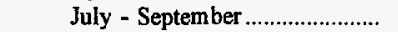 & 191,054 & 7,426 & 18,075 & 1,094 & 217,649 \\
\hline 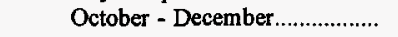 & 199,612 & 7,661 & 20,127 & 1,956 & 229,356 \\
\hline 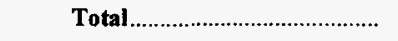 & 769,152 & 31,104 & 74,359 & 6,221 & 880,836 \\
\hline 1994 January - March ...................... & 199,981 & 7,318 & 17,990 & 2,016 & 227,305 \\
\hline April - June & 208,576 & 8,438 & 18,408 & 1,187 & 236,610 \\
\hline July - September & 212,849 & 7,881 & 18,777 & 1,135 & 240,642 \\
\hline October - December..................... & 210,523 & 8,081 & 19,717 & 1,674 & 239,996 \\
\hline 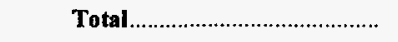 & 831,929 & 31,719 & 74,893 & 6,013 & 944,553 \\
\hline 1995 January - March .......................... & 205,054 & 8,261 & 17,871 & 1,638 & 232,824 \\
\hline April - June & 199,275 & 8,192 & 17,144 & 1,032 & 225,643 \\
\hline 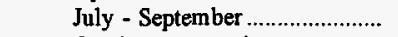 & 211,914 & 8,135 & 18,369 & 1,063 & 239,481 \\
\hline October - December................... & 210,617 & 8,449 & 18,510 & 2,074 & 239,649 \\
\hline 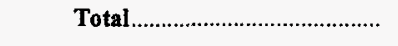 & 826,860 & 33,036 & 71,895 & 5,807 & 937,597 \\
\hline 1996 January - March ......................... & 204,394 & 7,894 & 17,444 & 1,802 & 231,534 \\
\hline April - June & 212,197 & 7,974 & 16,995 & 1,201 & 238,368 \\
\hline July - September .......................... & 226,454 & 8,187 & 17,598 & 1,201 & 253,440 \\
\hline October - December..................... & 219,656 & 7,616 & 18,804 & 1,802 & 247,878 \\
\hline 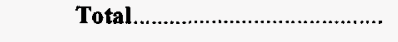 & 862,701 & 31,672 & $\mathbf{7 0 , 8 4 2}$ & 6,006 & 971,220 \\
\hline 1997 January - March ....................... & 213,527 & 7,330 & 17,352 & 1,939 & 240,148 \\
\hline 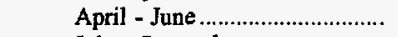 & 215,223 & 7,080 & 17,280 & 1,293 & 240,875 \\
\hline 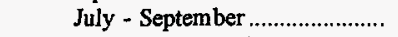 & 225,508 & 7,694 & 17,410 & 1,293 & 251,904 \\
\hline October - December ................... & 226,329 & 7,447 & 18,570 & 1,939 & 254,285 \\
\hline 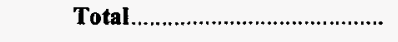 & 880,588 & 29,550 & 70,612 & 6,463 & 987,213 \\
\hline 1998 January - March ......................... & 225,000 & 6,840 & 17,213 & 1,939 & 250,992 \\
\hline 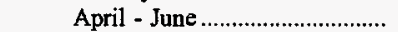 & 227,349 & 6,972 & 18,614 & 1,293 & 254,227 \\
\hline Total & 452,348 & 13,812 & 35,827 & 3,232 & 505,220 \\
\hline
\end{tabular}

Notes: Total may not equal sum of components because of independent rounding.

Sources: Energy Information Administration - Electric Utilities: FERC Form 423, "Monthly Report of Cost and Quality of Fuels for Electric Plants" Coke Plants: Form EIA-5, "Coke Plant Report - Quarterly" - Other Industrial: Form EIA-3, "Quarterly Coal Consumption Report-Manufacturing Plants;" Form EIA-6, "Coal Distribution Report;" Form EIA-867, "Annual Nonutility Power Producer Report;" Form EIA-7A, "Coal Production Report;" and - Residential and Commercial: Form EIA-6, "Coal Distribution Report." 
Table 21. Average Price of U.S. Coal Receipts by End-Use Sector, 1992-1998

(Dollars per Short Ton)

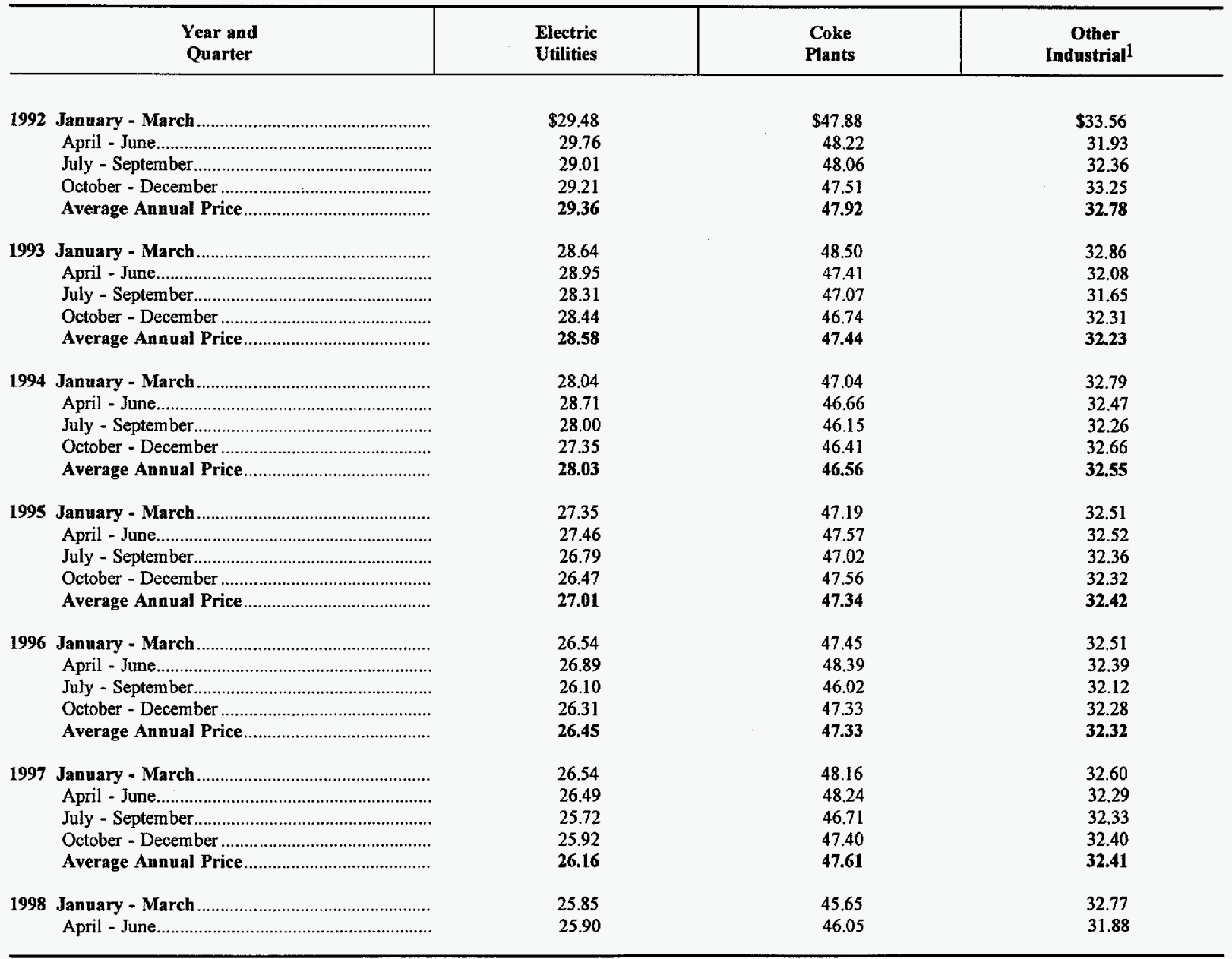

1 Manufacturing plants only.

Notes: Total may not equal sum of components because of independent rounding. Average price is based on the cost, including insurance and freight (c.i.f. cost). Price data for the Residential and Commercial sector are not available. See Technical Note 1 in Appendix $C$

Sources: Energy Information Administration (EIA) - Electric Utilities: Federal Energy Regulatory Commission (FERC), FERC Form 423,

"Monthly Report of Cost and Quality of Fuels for Electric Plants" - Coke Plants: Form EIA-5, "Coke Plant Report - Quarterly" and - Other Industrial: Form EIA-3, "Quarterly Coal Consumption Report - Manufacturing Plants." 
Table 22. Coal Receipts by Census Division and State (Thousand Short Tons)

\begin{tabular}{|c|c|c|c|c|c|c|}
\hline \multirow{2}{*}{$\begin{array}{l}\text { Census Division } \\
\text { and State }\end{array}$} & \multirow{2}{*}{$\begin{array}{l}\text { April - } \\
\text { June } \\
1998\end{array}$} & \multirow{2}{*}{$\begin{array}{c}\text { January - } \\
\text { March } \\
1998\end{array}$} & \multirow{2}{*}{$\begin{array}{l}\text { April - } \\
\text { June } \\
1997\end{array}$} & \multicolumn{3}{|c|}{ Year to Date } \\
\hline & & & & 1998 & 1997 & $\begin{array}{l}\text { Percent } \\
\text { Change }\end{array}$ \\
\hline New England Total.................................. & 1,762 & 2,027 & 1,969 & 3,789 & 3,725 & 1.7 \\
\hline 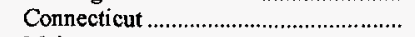 & 175 & 280 & 332 & 456 & 552 & -17.4 \\
\hline Maine & 27 & 38 & 58 & 65 & 102 & -36.0 \\
\hline 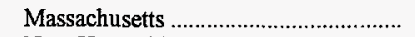 & 1,205 & 1,309 & 1,206 & 2,514 & 2,271 & 10.7 \\
\hline 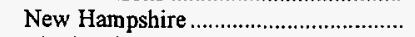 & 327 & 371 & 372 & 698 & 798 & -12.5 \\
\hline 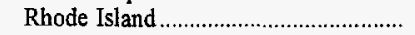 & 1 & 1 & 1 & 1 & 1 & .0 \\
\hline Vermont & 27 & 28 & 1 & 55 & 1 & $\mathrm{NM}$ \\
\hline 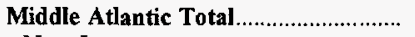 & 17,464 & 17,905 & 17,474 & 35,369 & 35,868 & -1.4 \\
\hline 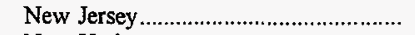 & 553 & 415 & 557 & 969 & 1,129 & -14.2 \\
\hline 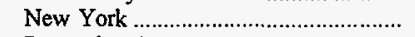 & 3,086 & 2,667 & 2,438 & 5,753 & 5,096 & 12.9 \\
\hline 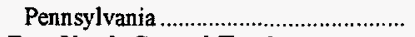 & 13,825 & 14,823 & 14,479 & 28,648 & 29,644 & -3.4 \\
\hline East North Central Total ...................... & 59,644 & 55,577 & 58,754 & 115,221 & 112,164 & 2.7 \\
\hline 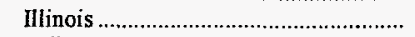 & 11,187 & 11,668 & 11,925 & 22,855 & 24,240 & -5.7 \\
\hline 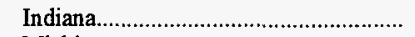 & 16,482 & 16,883 & 16,209 & 33,365 & 31,341 & 6.5 \\
\hline 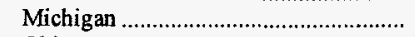 & 10,730 & 6,426 & 9,329 & 17,156 & 15,101 & 13.6 \\
\hline Ohio & 14,570 & 14,980 & 14,312 & 29,549 & 28,989 & 1.9 \\
\hline Wisconsin & 6,676 & 5,621 & 6,979 & 12,296 & 12,493 & -1.6 \\
\hline West North Central Total .................... & 35,417 & 36,611 & 30,699 & 72,028 & 64,769 & 11.2 \\
\hline Iowa & 6,374 & 5,328 & 4,923 & 11,702 & 9,932 & 17.8 \\
\hline 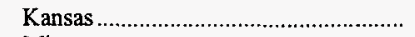 & 4,680 & 4,779 & 3,515 & 9,459 & 8,034 & 17.7 \\
\hline Minnesota & 4,287 & 5,073 & 3,747 & 9,359 & 9,012 & 3.9 \\
\hline 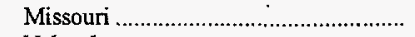 & 9,849 & 9,832 & 8,355 & 19,681 & 16,671 & 18.1 \\
\hline 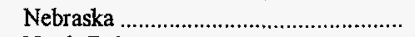 & 2,985 & 3,049 & 2,635 & 6,034 & 5,538 & 9.0 \\
\hline 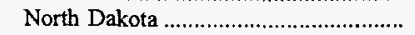 & 6,653 & 7,990 & 6,905 & 14,643 & 14,423 & 1.5 \\
\hline 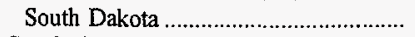 & 589 & 560 & 621 & 1,149 & 1,158 & -.8 \\
\hline 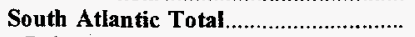 & 42,951 & 42,900 & 40,337 & $\mathbf{8 5 , 8 5 1}$ & 81,239 & 5.7 \\
\hline Delaware & 378 & 422 & 500 & 801 & 955 & -16.2 \\
\hline 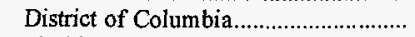 & 8 & 12 & 8 & 20 & 20 & .0 \\
\hline 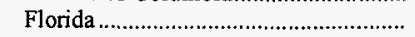 & 7,455 & 7,073 & 7,383 & 14,528 & 14,215 & 2.2 \\
\hline 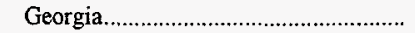 & 7,881 & 7,970 & 7,581 & 15,851 & 14,679 & 8.0 \\
\hline Maryland & 2,885 & 2,997 & 2,597 & 5,882 & 5,313 & 10.7 \\
\hline 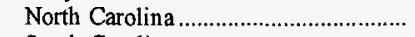 & 7,135 & 7,549 & 6,959 & 14,685 & 14,384 & 2.1 \\
\hline 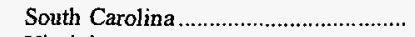 & 3,846 & 3,620 & 3,339 & 7,466 & 6,753 & 10.6 \\
\hline Virginia & 3,870 & 3,913 & 3,513 & 7,783 & 7,604 & 2.4 \\
\hline West Virginia......................................... & 9,494 & 9,343 & 8,456 & 18,837 & 17,315 & 8.8 \\
\hline East South Central Total & 27,554 & 28,834 & 29,460 & 56,388 & 57,243 & -1.5 \\
\hline Alabama & 8,531 & 8,793 & 9,237 & 17,324 & 18,440 & -6.1 \\
\hline Kentucky & 10,336 & 10,576 & 11,177 & 20,912 & 21,280 & -1.7 \\
\hline 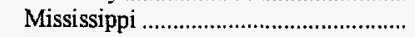 & 1,655 & 1,504 & 1,544 & 3,159 & 2,996 & 5.5 \\
\hline 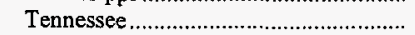 & 7,031 & 7,962 & 7,501 & 14,993 & 14,527 & 3.2 \\
\hline West South Central Total........................ & 37,125 & 35,050 & $\mathbf{3 4 , 5 7 2}$ & 72,175 & 69,539 & 3.8 \\
\hline 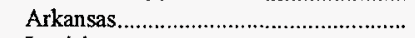 & 3,603 & 3,119 & 2,900 & 6,722 & 6,033 & 11.4 \\
\hline 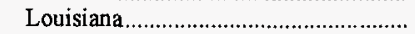 & 3,191 & 3,287 & 3,440 & 6,478 & 6,552 & -1.1 \\
\hline Oklahoma & 5,361 & 5,341 & 4,897 & 10,702 & 9,847 & 8.7 \\
\hline 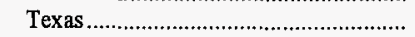 & 24,970 & 23,303 & 23,336 & 48,272 & 47,106 & 2.5 \\
\hline Mountain Total. & 28,007 & 29,304 & 25,928 & $\mathbf{5 7 , 3 1 1}$ & 52,961 & 8.2 \\
\hline Arizona & 4,827 & 4,645 & 4,312 & 9,472 & 8,019 & 18.1 \\
\hline 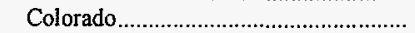 & 4,591 & 4,622 & 4,456 & 9,213 & 8,559 & 7.6 \\
\hline 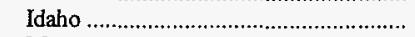 & 73 & 117 & 69 & 190 & 200 & -4.7 \\
\hline 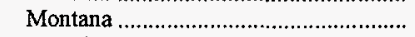 & 2,317 & 2,682 & 1,693 & 5,000 & 4,006 & 24.8 \\
\hline 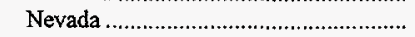 & 1,472 & 2,226 & 1,535 & 3,699 & 3,353 & 10.3 \\
\hline 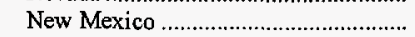 & 3,692 & 3,636 & 3,918 & 7,328 & 7,970 & -8.1 \\
\hline 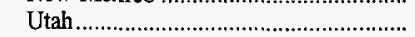 & 4,365 & 4,292 & 4,316 & 8,657 & 8,706 & -.6 \\
\hline 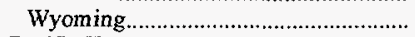 & 6,668 & 7,084 & 5,630 & 13,752 & 12,148 & 13.2 \\
\hline Pacific Total & 4,303 & 2,784 & 1,683 & 7,087 & $3, \mathbf{5 1 5}$ & 101.6 \\
\hline 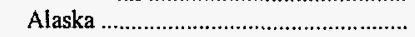 & 101 & 151 & 101 & 252 & 253 & -.2 \\
\hline 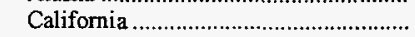 & 699 & 724 & 543 & 1,423 & 1,050 & 35.5 \\
\hline 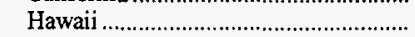 & 5 & 70 & 30 & 76 & 84 & -10.3 \\
\hline Oregon & 1,779 & 692 & 2 & 2,471 & 152 & $\mathrm{NM}$ \\
\hline 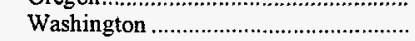 & 1,719 & 1,146 & 1,006 & 2,865 & 1,976 & 45.0 \\
\hline 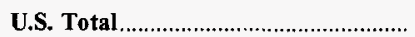 & 254,227 & 250,992 & 240,875 & 505,220 & 481,023 & $\mathbf{5 . 0}$ \\
\hline
\end{tabular}

NM Percent change calculation not meaningful as value is greater than 500 .

Notes: Total may not equal sum of components because of independent rounding.

Sources: Energy Information Administration, FERC Form 423, "Monthly Report of Cost and Quality of Fuels for Electric Plants," Form EIA-3, "Quarterly Coal Consumption-Manufacturing Plants;" Form EIA-5, "Coke Plant Report - Quarterly;" Form EIA-867, "Annual Nonutility Power Producer Report;" Form EIA-7A, "Coal Production Report;" and Form EIA-6, "Coal Distribution Report." 
Table 23. Quantity and Price of Coal Receipts at Electric Utility Plants by Census Division and State

\begin{tabular}{|c|c|c|c|c|c|c|c|c|c|c|}
\hline \multirow{3}{*}{$\begin{array}{l}\text { Census Division } \\
\text { and State }\end{array}$} & \multicolumn{2}{|c|}{$\begin{array}{c}\text { April-June } \\
1998\end{array}$} & \multicolumn{2}{|c|}{$\begin{array}{c}\text { April-June } \\
1997\end{array}$} & \multicolumn{6}{|c|}{ Year to Date } \\
\hline & \multirow[b]{2}{*}{$\begin{array}{l}\text { Quantity } \\
\text { (thousand } \\
\text { short } \\
\text { tons) }\end{array}$} & \multirow[b]{2}{*}{$\begin{array}{c}\text { Price } \\
\text { (cents per } \\
\text { MM Btu) }\end{array}$} & \multirow[b]{2}{*}{$\begin{array}{l}\text { Quantity } \\
\text { (thousand } \\
\text { short } \\
\text { tons) }\end{array}$} & \multirow[b]{2}{*}{$\begin{array}{c}\text { Price } \\
\text { (cents per } \\
\text { MM Btu) }\end{array}$} & \multicolumn{2}{|c|}{1998} & \multicolumn{2}{|c|}{1997} & \multicolumn{2}{|c|}{ Percent Change } \\
\hline & & & & & $\begin{array}{l}\text { Quantity } \\
\text { (thousand } \\
\text { short } \\
\text { tons) }\end{array}$ & $\begin{array}{c}\text { Price } \\
\text { (cents } \\
\text { per } \\
\text { MM Btu) }\end{array}$ & $\begin{array}{l}\text { Quantity } \\
\text { (thousand } \\
\text { short } \\
\text { tons) }\end{array}$ & $\begin{array}{c}\text { Price } \\
\text { (cents } \\
\text { per } \\
\text { MM Btu) }\end{array}$ & Quantity & Price \\
\hline 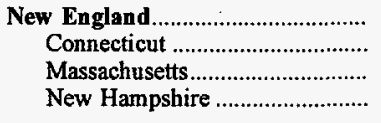 & $\begin{array}{r}1,640 \\
174 \\
1,190 \\
276\end{array}$ & $\begin{array}{l}166 \\
177 \\
166 \\
160\end{array}$ & $\begin{array}{r}1,894 \\
331 \\
1,193 \\
370\end{array}$ & $\begin{array}{l}170 \\
192 \\
167 \\
160\end{array}$ & $\begin{array}{r}3,580 \\
452 \\
2,482 \\
645\end{array}$ & $\begin{array}{l}168 \\
181 \\
168 \\
161\end{array}$ & $\begin{array}{r}3,586 \\
548 \\
2,243 \\
795\end{array}$ & $\begin{array}{l}172 \\
192 \\
171 \\
161\end{array}$ & $\begin{array}{r}-0.2 \\
-17.5 \\
10.7 \\
-18.9\end{array}$ & $\begin{array}{r}-2.4 \\
-5.8 \\
-2.2 \\
.1\end{array}$ \\
\hline 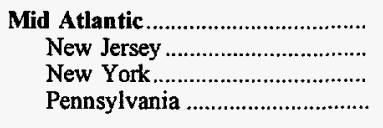 & $\begin{array}{r}13,600 \\
549 \\
2,315 \\
10,736\end{array}$ & $\begin{array}{l}138 \\
157 \\
142 \\
136\end{array}$ & $\begin{array}{r}13,026 \\
553 \\
1,691 \\
10,782\end{array}$ & $\begin{array}{l}137 \\
177 \\
143 \\
133\end{array}$ & $\begin{array}{r}27,096 \\
960 \\
4,300 \\
21,836\end{array}$ & $\begin{array}{l}138 \\
161 \\
144 \\
136\end{array}$ & $\begin{array}{r}26,645 \\
1,121 \\
3,643 \\
21,881\end{array}$ & $\begin{array}{l}139 \\
176 \\
141 \\
137\end{array}$ & $\begin{array}{r}1.7 \\
-14.4 \\
18.0 \\
-.2\end{array}$ & $\begin{array}{r}-.4 \\
-8.8 \\
2.0 \\
-.3\end{array}$ \\
\hline 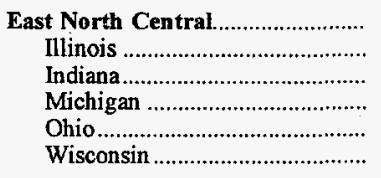 & $\begin{array}{r}\mathbf{5 2 , 6 0 3} \\
9,482 \\
13,825 \\
9,874 \\
13,152 \\
6,271\end{array}$ & $\begin{array}{l}131 \\
165 \\
112 \\
132 \\
137 \\
110\end{array}$ & $\begin{array}{r}51,653 \\
10,352 \\
13,429 \\
8,502 \\
12,872 \\
6,498\end{array}$ & $\begin{array}{l}131 \\
156 \\
115 \\
140 \\
132 \\
111\end{array}$ & $\begin{array}{r}101,533 \\
19,467 \\
28,128 \\
15,875 \\
26,575 \\
11,488\end{array}$ & $\begin{array}{l}131 \\
160 \\
113 \\
132 \\
137 \\
107\end{array}$ & $\begin{array}{l}98,192 \\
21,043 \\
25,817 \\
13,846 \\
25,886 \\
11,600\end{array}$ & $\begin{array}{l}133 \\
164 \\
116 \\
137 \\
132 \\
109\end{array}$ & $\begin{array}{r}3.4 \\
-7.5 \\
9.0 \\
14.6 \\
2.7 \\
-1.0\end{array}$ & $\begin{array}{r}-1.7 \\
-2.4 \\
-3.1 \\
-4.4 \\
3.4 \\
-1.6\end{array}$ \\
\hline 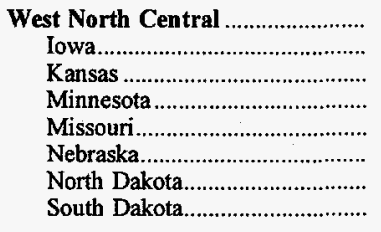 & $\begin{array}{r}31,929 \\
5,434 \\
4,652 \\
3,869 \\
9,510 \\
2,906 \\
5,082 \\
477\end{array}$ & $\begin{array}{r}91 \\
92 \\
99 \\
110 \\
92 \\
59 \\
80 \\
92\end{array}$ & $\begin{array}{r}27,366 \\
4,092 \\
3,481 \\
3,402 \\
8,005 \\
2,545 \\
5,327 \\
513\end{array}$ & $\begin{array}{r}93 \\
94 \\
111 \\
113 \\
94 \\
61 \\
75 \\
93\end{array}$ & $\begin{array}{r}65,223 \\
10,070 \\
9,398 \\
8,567 \\
18,968 \\
5,858 \\
11,423 \\
939\end{array}$ & $\begin{array}{r}90 \\
90 \\
99 \\
111 \\
92 \\
59 \\
77 \\
93\end{array}$ & $\begin{array}{r}\mathbf{5 8 , 0 1 9} \\
\mathbf{8 , 3 7 8} \\
7,963 \\
8,275 \\
15,910 \\
5,349 \\
11,199 \\
945\end{array}$ & $\begin{array}{r}92 \\
92 \\
106 \\
112 \\
94 \\
60 \\
76 \\
93\end{array}$ & $\begin{array}{r}12.4 \\
20.2 \\
18.0 \\
3.5 \\
19.2 \\
9.5 \\
2.0 \\
-.6\end{array}$ & $\begin{array}{r}-2.3 \\
-2.7 \\
-7.1 \\
-1.2 \\
-2.4 \\
-1.6 \\
1.5 \\
-.4\end{array}$ \\
\hline 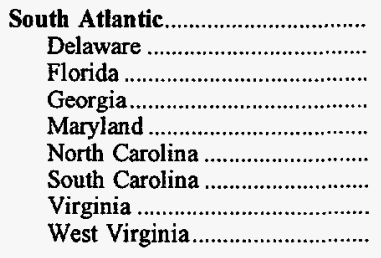 & $\begin{array}{r}39,180 \\
328 \\
7,098 \\
7,402 \\
2,699 \\
6,601 \\
3,346 \\
3,043 \\
8,662\end{array}$ & $\begin{array}{l}145 \\
160 \\
164 \\
154 \\
146 \\
145 \\
144 \\
138 \\
122\end{array}$ & $\begin{array}{r}36,693 \\
458 \\
7,041 \\
7,104 \\
2,390 \\
6,400 \\
2,858 \\
2,598 \\
7,845\end{array}$ & $\begin{array}{l}148 \\
157 \\
173 \\
159 \\
151 \\
142 \\
144 \\
139 \\
124\end{array}$ & $\begin{array}{r}78,110 \\
730 \\
13,839 \\
14,804 \\
5,490 \\
13,529 \\
6,484 \\
6,010 \\
17,224\end{array}$ & $\begin{array}{l}145 \\
157 \\
167 \\
155 \\
146 \\
144 \\
145 \\
138 \\
122\end{array}$ & $\begin{array}{r}73,506 \\
868 \\
13,537 \\
13,657 \\
4,896 \\
13,119 \\
5,734 \\
5,700 \\
15,994\end{array}$ & $\begin{array}{l}148 \\
161 \\
175 \\
159 \\
152 \\
143 \\
145 \\
139 \\
124\end{array}$ & $\begin{array}{r}6.3 \\
-15.9 \\
2.2 \\
8.4 \\
12.1 \\
3.1 \\
13.1 \\
5.4 \\
7.7\end{array}$ & $\begin{array}{r}-2.1 \\
-2.2 \\
-4.9 \\
-2.4 \\
-4.1 \\
.7 \\
-.4 \\
-.5 \\
-1.2\end{array}$ \\
\hline 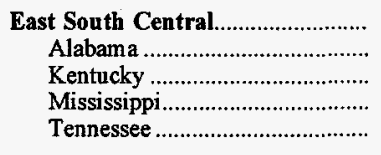 & $\begin{array}{r}24,436 \\
7,254 \\
9,477 \\
1,616 \\
6,088\end{array}$ & $\begin{array}{l}125 \\
157 \\
105 \\
152 \\
112\end{array}$ & $\begin{array}{r}26,074 \\
7,800 \\
10,208 \\
1,491 \\
6,575\end{array}$ & $\begin{array}{l}123 \\
152 \\
104 \\
156 \\
113\end{array}$ & $\begin{array}{r}49,889 \\
14,678 \\
19,105 \\
3,058 \\
13,048\end{array}$ & $\begin{array}{l}125 \\
156 \\
105 \\
153 \\
113\end{array}$ & $\begin{array}{r}\mathbf{5 0 , 3 5 2} \\
15,535 \\
19,308 \\
2,893 \\
12,616\end{array}$ & $\begin{array}{l}124 \\
153 \\
104 \\
154 \\
113\end{array}$ & $\begin{array}{r}-.9 \\
-5.5 \\
-1.1 \\
5.7 \\
3.4\end{array}$ & $\begin{array}{r}.5 \\
2.2 \\
1.0 \\
-.6 \\
-.6\end{array}$ \\
\hline 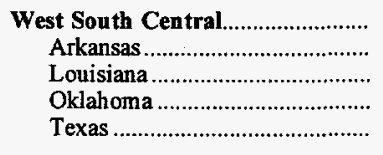 & $\begin{array}{r}35,544 \\
3,530 \\
3,184 \\
5,110 \\
23,719\end{array}$ & $\begin{array}{r}125 \\
150 \\
142 \\
92 \\
127\end{array}$ & $\begin{array}{r}33,153 \\
2,824 \\
3,428 \\
4,674 \\
22,227\end{array}$ & $\begin{array}{r}130 \\
171 \\
149 \\
93 \\
130\end{array}$ & $\begin{array}{r}69,030 \\
6,580 \\
6,451 \\
10,144 \\
45,855\end{array}$ & $\begin{array}{r}127 \\
149 \\
142 \\
92 \\
129\end{array}$ & $\begin{array}{r}66,585 \\
5,865 \\
6,506 \\
9,366 \\
44,847\end{array}$ & $\begin{array}{r}129 \\
169 \\
150 \\
93 \\
128\end{array}$ & $\begin{array}{r}3.7 \\
12.2 \\
-.8 \\
8.3 \\
2.2\end{array}$ & $\begin{array}{r}-1.4 \\
-11.4 \\
-5.3 \\
-.5 \\
1.3\end{array}$ \\
\hline 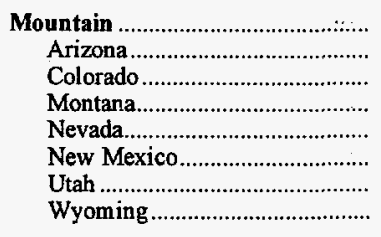 & $\begin{array}{r}26,411 \\
4,632 \\
4,420 \\
2,291 \\
1,421 \\
3,671 \\
3,791 \\
6,186\end{array}$ & $\begin{array}{r}110 \\
135 \\
101 \\
76 \\
153 \\
136 \\
118 \\
71\end{array}$ & $\begin{array}{r}24,406 \\
4,160 \\
4,248 \\
1,655 \\
1,497 \\
3,896 \\
3,826 \\
5,124\end{array}$ & $\begin{array}{r}114 \\
146 \\
105 \\
69 \\
152 \\
133 \\
109 \\
80\end{array}$ & $\begin{array}{r}\mathbf{5 4 , 0 9 5} \\
9,104 \\
\mathbf{8 , 8 4 3} \\
4,921 \\
3,594 \\
7,290 \\
7,638 \\
12,705\end{array}$ & $\begin{array}{r}108 \\
134 \\
100 \\
71 \\
140 \\
133 \\
115 \\
75\end{array}$ & $\begin{array}{r}49,856 \\
7,680 \\
8,140 \\
3,894 \\
3,268 \\
7,927 \\
7,837 \\
11,111\end{array}$ & $\begin{array}{r}113 \\
147 \\
104 \\
69 \\
142 \\
135 \\
112 \\
81\end{array}$ & $\begin{array}{r}8.5 \\
18.5 \\
8.6 \\
26.4 \\
10.0 \\
-8.0 \\
-2.5 \\
14.3\end{array}$ & $\begin{array}{r}-4.1 \\
-8.9 \\
-3.8 \\
4.1 \\
-1.8 \\
-1.2 \\
2.9 \\
-7.3\end{array}$ \\
\hline 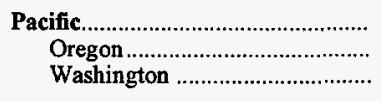 & $\begin{array}{r}2,005 \\
304 \\
1,701\end{array}$ & $\begin{array}{l}137 \\
110 \\
142\end{array}$ & $\begin{array}{r}958 \\
- \\
958\end{array}$ & $\begin{array}{r}191 \\
- \\
191\end{array}$ & $\begin{array}{r}3,792 \\
965 \\
2,827\end{array}$ & $\begin{array}{l}140 \\
109 \\
151\end{array}$ & $\begin{array}{r}2,009 \\
135 \\
1,874\end{array}$ & $\begin{array}{l}187 \\
114 \\
193\end{array}$ & $\begin{array}{c}\mathbf{8 8 . 7} \\
\mathrm{NM} \\
50.8\end{array}$ & $\begin{array}{r}-25.4 \\
-4.4 \\
-21.9\end{array}$ \\
\hline U.S. Total. & 227,349 & 126 & 215,223 & 129 & 452,348 & 126 & 428,751 & 129 & 5.5 & -2.0 \\
\hline
\end{tabular}

NM Percent change calculation not meaningful as value is greater than 500

Notes: Total may not equal sum of components because of independent rounding. MM Btu represents million Btu. Source: Federal Energy Regulatory Commission, FERC Form 423, "Monthly Report of Cost and Quality of Fuels for Electric Plants." 
Table 24. Quantity and Price of Contract Coal Receipts at Electric Utility Plants by Census Division and State

\begin{tabular}{|c|c|c|c|c|c|c|c|c|c|c|}
\hline \multirow{3}{*}{$\begin{array}{c}\text { Census Division } \\
\text { and State }\end{array}$} & \multicolumn{2}{|c|}{$\begin{array}{c}\text { April-June } \\
1998\end{array}$} & \multicolumn{2}{|c|}{$\begin{array}{c}\text { April-June } \\
1997\end{array}$} & \multicolumn{6}{|c|}{ Year to Date } \\
\hline & \multirow[b]{2}{*}{$\begin{array}{c}\text { Quantity } \\
\text { (thousand } \\
\text { short } \\
\text { tons) }\end{array}$} & \multirow[b]{2}{*}{$\begin{array}{c}\text { Price } \\
\text { (cents per } \\
\text { MM Btu) }\end{array}$} & \multirow[b]{2}{*}{$\begin{array}{c}\text { Quantity } \\
\text { (thousand } \\
\text { short } \\
\text { tons) }\end{array}$} & \multirow[b]{2}{*}{$\begin{array}{c}\text { Price } \\
\text { (cents per } \\
\text { MM Btu) }\end{array}$} & \multicolumn{2}{|c|}{1998} & \multicolumn{2}{|c|}{1997} & \multicolumn{2}{|c|}{ Percent Change } \\
\hline & & & & & $\begin{array}{l}\text { Quantity } \\
\text { (thousand } \\
\text { short } \\
\text { tons) }\end{array}$ & $\begin{array}{c}\text { Price } \\
\text { (cents } \\
\text { per } \\
\text { MM Btu) }\end{array}$ & $\begin{array}{c}\text { Quantity } \\
\text { (thousand } \\
\text { short } \\
\text { tons) }\end{array}$ & 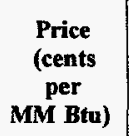 & Quantity & Price \\
\hline 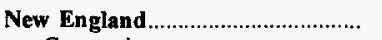 & 1,298 & 166 & 1,643 & 171 & 2,980 & 169 & 3,005 & 173 & -0.8 & -2.2 \\
\hline 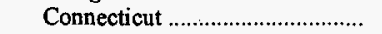 & 110 & 184 & 312 & 193 & 358 & 185 & 515 & 193 & -30.5 & -4.0 \\
\hline Massachusetts.................................. & 1,001 & 165 & 1,112 & 167 & 2,174 & 167 & 2,019 & 170 & 7.7 & -1.9 \\
\hline New Hampshire ........................... & 187 & 165 & 219 & 160 & 448 & 164 & 471 & 160 & -4.9 & 2.4 \\
\hline Mid Atlantic & 11,352 & 141 & 9,958 & 140 & 22,642 & 141 & 20,639 & 142 & 9.7 & -.7 \\
\hline 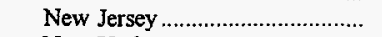 & 459 & 160 & 506 & 178 & 727 & 164 & 1,067 & 177 & -31.8 & -7.0 \\
\hline 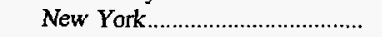 & 1,864 & 142 & 1,397 & 138 & 3,742 & 144 & 3,185 & 138 & 17.5 & 4.1 \\
\hline 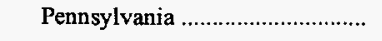 & 9,029 & 139 & 8,054 & 138 & 18,172 & 140 & 16,386 & 141 & 10.9 & -.7 \\
\hline East North Central........................... & 40,673 & 137 & 40,037 & 137 & $\mathbf{7 7 , 4 2 5}$ & 136 & 75,190 & 140 & 3.0 & -2.5 \\
\hline 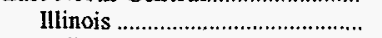 & 8,729 & 169 & 8,623 & 163 & 17,068 & 166 & 17,641 & 173 & -3.2 & -4.1 \\
\hline 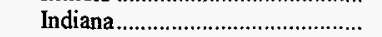 & 9,770 & 116 & 9,726 & 122 & 20,038 & 117 & 18,517 & 124 & 8.2 & -5.5 \\
\hline 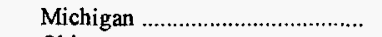 & 7,834 & 133 & 7,451 & 141 & 12,160 & 132 & 11,467 & 138 & 6.0 & -4.1 \\
\hline Ohio & 9,777 & 145 & 8,871 & 144 & 19,363 & 147 & 18,032 & 143 & 7.4 & 2.5 \\
\hline 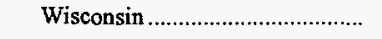 & 4,563 & 104 & 5,366 & 106 & 8,795 & 102 & 9,534 & 104 & -7.7 & -2.4 \\
\hline 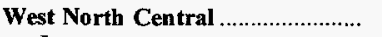 & 26,215 & 91 & 24,726 & 93 & 54,160 & 90 & 52,220 & 93 & 3.7 & -3.5 \\
\hline Iowa & 4,331 & 95 & 3,346 & 95 & 7,692 & 91 & 6,834 & 93 & 12.6 & -2.1 \\
\hline 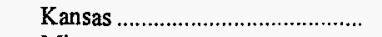 & 4,628 & 99 & 3,475 & 111 & 9,319 & 99 & 7,899 & 106 & 18.0 & -7.1 \\
\hline 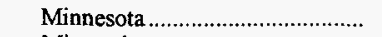 & 3,714 & 110 & 3,204 & 112 & 8,332 & 110 & 8,007 & 112 & 4.1 & -1.5 \\
\hline 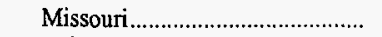 & 5,857 & 90 & 6,954 & 95 & 11,747 & 89 & 13,523 & 94 & -13.1 & -5.7 \\
\hline 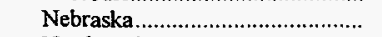 & 2,125 & 55 & 1,919 & 57 & 4,707 & 56 & 4,022 & 57 & 17.0 & -2.2 \\
\hline 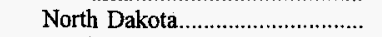 & 5,082 & 80 & 5,315 & 75 & 11,423 & 77 & 10,991 & 76 & 3.9 & 1.6 \\
\hline 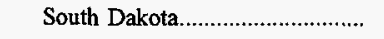 & 477 & 92 & 513 & 93 & 939 & 93 & 945 & 93 & -.6 & -.4 \\
\hline South Atlantic... & 28,055 & 146 & 26,972 & 148 & 55,616 & 147 & 53,875 & 149 & 3.2 & -1.5 \\
\hline 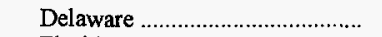 & 316 & 160 & 379 & 160 & 715 & 157 & 679 & 163 & 5.2 & -3.5 \\
\hline Florida & 4,734 & 170 & 4,501 & 181 & 9,165 & 174 & 8,737 & 182 & 4.9 & -4.8 \\
\hline 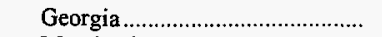 & 3,682 & 158 & 3,717 & 167 & 7,295 & 160 & 7,598 & 166 & -4.0 & -3.5 \\
\hline 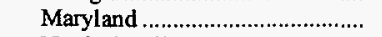 & 1,998 & 145 & 1,991 & 148 & 3,872 & 146 & 3,747 & 149 & 3.3 & -1.7 \\
\hline 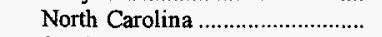 & 5,248 & 149 & 4,834 & 146 & 10,083 & 148 & 9,661 & 146 & 4.4 & 1.4 \\
\hline 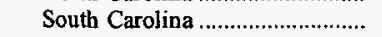 & 2,636 & 144 & 2,092 & 144 & 5,247 & 144 & 4,297 & 146 & 22.1 & -1.0 \\
\hline 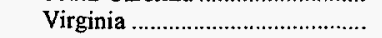 & 1,927 & 139 & 2,012 & 139 & 4,173 & 139 & 4,336 & 139 & -3.7 & .5 \\
\hline 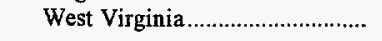 & 7,512 & 125 & 7,447 & 125 & 15,065 & 125 & 14,820 & 125 & 1.7 & -.5 \\
\hline East South Central............................ & 18,824 & 129 & 19,557 & 127 & 37,091 & 129 & 38,214 & 128 & -2.9 & 1.1 \\
\hline 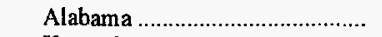 & 6,302 & 162 & 6,522 & 158 & 12,493 & 161 & 12,899 & 158 & -3.1 & 1.7 \\
\hline 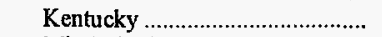 & 6,107 & 105 & 7,017 & 103 & 12,094 & 105 & 13,789 & 104 & -12.3 & 1.6 \\
\hline 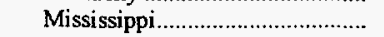 & 1,319 & 155 & 1,302 & 158 & 2,641 & 156 & 2,553 & 156 & 3.4 & -.1 \\
\hline 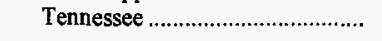 & 5,096 & 111 & 4,716 & 113 & 9,863 & 111 & 8,972 & 112 & 9.9 & -.9 \\
\hline West South Central ............................ & 33,411 & 125 & $\mathbf{3 1 , 5 5 1}$ & 131 & 64,819 & 127 & 63,384 & 129 & 2.3 & -1.4 \\
\hline 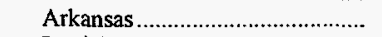 & 3,316 & 152 & 2,662 & 174 & 5,893 & 155 & 5,467 & 172 & 7.8 & -10.0 \\
\hline 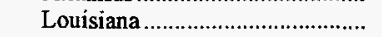 & 3,184 & 142 & 3,428 & 149 & 6,451 & 142 & 6,506 & 150 & -8 & -5.3 \\
\hline 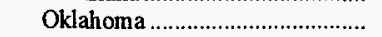 & 4,968 & 92 & 4,662 & 93 & 10,003 & 92 & 9,354 & 93 & 6.9 & -.5 \\
\hline 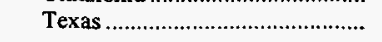 & 21,942 & 127 & 20,799 & 130 & 42,473 & 130 & 42,056 & 128 & 1.0 & 1.3 \\
\hline Mountain & 24,957 & 111 & 22,953 & 114 & $\mathbf{5 0 , 8 5 8}$ & 109 & 46,712 & 114 & 8.9 & -3.8 \\
\hline 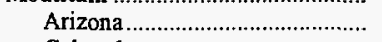 & 3,949 & 140 & 3,546 & 150 & 7,821 & 138 & 6,490 & 152 & 20.5 & -8.9 \\
\hline 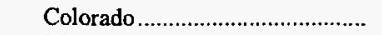 & 4,130 & 103 & 3,955 & 106 & 8,245 & 102 & 7,380 & 106 & 11.7 & -3.9 \\
\hline 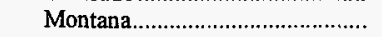 & 2,291 & 76 & 1,655 & 69 & 4,921 & 71 & 3,894 & 69 & 26.4 & 4.1 \\
\hline 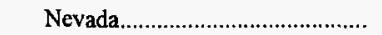 & 1,421 & 153 & 1,410 & 154 & 3,318 & 141 & 3,089 & 143 & 7.4 & -1.4 \\
\hline 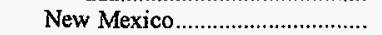 & 3,671 & 136 & 3,896 & 133 & 7,290 & 133 & 7,927 & 135 & -8.0 & -1.2 \\
\hline Utah & 3,706 & 118 & 3,473 & 111 & 7,497 & 116 & 7,283 & 114 & 2.9 & 1.7 \\
\hline 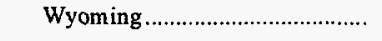 & 5,790 & 71 & 5,018 & 79 & 11,767 & 76 & 10,650 & 81 & 10.5 & -6.1 \\
\hline 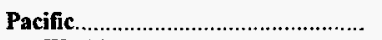 & 1,190 & 153 & 958 & 191 & 2,079 & 164 & 1,870 & 193 & 11.2 & -15.3 \\
\hline 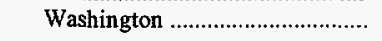 & 1,190 & 153 & 958 & 191 & 2,079 & 164 & 1,870 & 193 & 11.2 & -15.3 \\
\hline 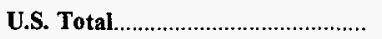 & 185,975 & 128 & 178,355 & 130 & 367,670 & 128 & 355,110 & 130 & 3.5 & -1.9 \\
\hline
\end{tabular}

Notes: Total may not equal sum of components because of independent rounding. MM Btu represents million Btu.

Source: Federal Energy Regulatory Commission, FERC Form 423, "Monthly Report of Cost and Quality of Fuels for Electric Plants." 
Table 25. Quantity and Price of Spot Coal Receipts at Electric Utility Plants by Census Division and State

\begin{tabular}{|c|c|c|c|c|c|c|c|c|c|c|}
\hline \multirow{3}{*}{$\begin{array}{c}\text { Census Division } \\
\text { and State }\end{array}$} & \multicolumn{2}{|c|}{$\begin{array}{c}\text { April-June } \\
1998\end{array}$} & \multicolumn{2}{|c|}{$\begin{array}{c}\text { April-June } \\
1997\end{array}$} & \multicolumn{6}{|c|}{ Year to Date } \\
\hline & \multirow[b]{2}{*}{$\begin{array}{c}\text { Quantity } \\
\text { (thousand } \\
\text { short } \\
\text { tons) }\end{array}$} & \multirow[b]{2}{*}{$\begin{array}{c}\text { Price } \\
\text { (cents per } \\
\text { MM Btu) }\end{array}$} & \multirow[b]{2}{*}{$\begin{array}{l}\text { Quantity } \\
\text { (thousand } \\
\text { short } \\
\text { tons) }\end{array}$} & \multirow[b]{2}{*}{$\begin{array}{c}\text { Price } \\
\text { (cents per } \\
\text { MM Btu) }\end{array}$} & \multicolumn{2}{|c|}{1998} & \multicolumn{2}{|c|}{1997} & \multicolumn{2}{|c|}{ Percent Change } \\
\hline & & & & & $\begin{array}{c}\text { Quantity } \\
\text { (thousand } \\
\text { short } \\
\text { tons) }\end{array}$ & 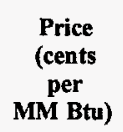 & $\begin{array}{c}\text { Quantity } \\
\text { (thousand } \\
\text { short } \\
\text { tons) }\end{array}$ & $\begin{array}{c}\text { Price } \\
\text { (cents } \\
\text { per } \\
\text { MM Btu) }\end{array}$ & Quantity & Price \\
\hline 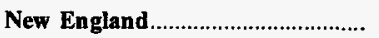 & 342 & 165 & 251 & 166 & 600 & 165 & 581 & 170 & 3.2 & $-\mathbf{3 . 0}$ \\
\hline 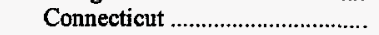 & 64 & 164 & 19 & 179 & 94 & 166 & 33 & 182 & 184.8 & -8.5 \\
\hline 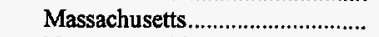 & 190 & 173 & 81 & 170 & 309 & 173 & 224 & 183 & 37.8 & -5.1 \\
\hline 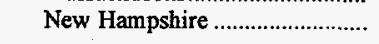 & 89 & 148 & 151 & 161 & 197 & 153 & 324 & 161 & -39.2 & -5.0 \\
\hline Mid Atlantic & 2,248 & 123 & 3,069 & 124 & 4,454 & 123 & 6,006 & 127 & -25.8 & -2.9 \\
\hline 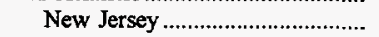 & 89 & 136 & 47 & 166 & 233 & 148 & 54 & 166 & 331.6 & -10.7 \\
\hline 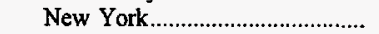 & 451 & 143 & 294 & 164 & 558 & 147 & 458 & 164 & 21.9 & -10.0 \\
\hline 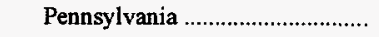 & 1,707 & 117 & 2,728 & 119 & 3,664 & 118 & 5,494 & 123 & -33.3 & -4.3 \\
\hline East North Central.............................. & 11,930 & 114 & 11,616 & 111 & 24,108 & 113 & 23,002 & 112 & 4.8 & 1.0 \\
\hline 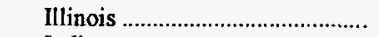 & 753 & 116 & 1,729 & 124 & 2,399 & 122 & 3,402 & 125 & -29.5 & -2.4 \\
\hline 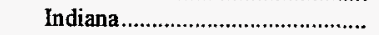 & 4,055 & 102 & 3,703 & 97 & 8,089 & 103 & 7,300 & 99 & 10.8 & 4.1 \\
\hline 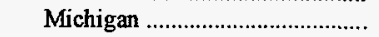 & 2,040 & 129 & 1,052 & 135 & 3,715 & 129 & 2,380 & 135 & 56.1 & -4.6 \\
\hline 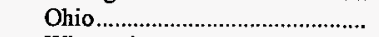 & 3,375 & 113 & 4,001 & 106 & 7,212 & 110 & 7,853 & 107 & -8.2 & 2.3 \\
\hline Wisconsin & 1,708 & 122 & 1,132 & 131 & 2,693 & 122 & 2,066 & 127 & 30.3 & -3.7 \\
\hline West North Central ........................... & 5,714 & 90 & 2,639 & 89 & 11,063 & 92 & 5,799 & 86 & 90.8 & 6.6 \\
\hline 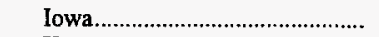 & 1,103 & 81 & 746 & 93 & 2,378 & 87 & 1,544 & 90 & 54.0 & -3.9 \\
\hline 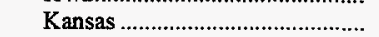 & 24 & 64 & 6 & 119 & 79 & 64 & 64 & 73 & 23.3 & -11.9 \\
\hline 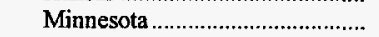 & 154 & 125 & 198 & 114 & 235 & 125 & 268 & 117 & -12.4 & 6.9 \\
\hline 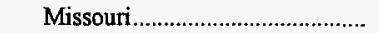 & 3,652 & 95 & 1,051 & 91 & 7,220 & 96 & 2,387 & 92 & 202.5 & 4.7 \\
\hline 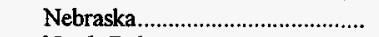 & 781 & 70 & 626 & 71 & 1,151 & 69 & 1,328 & 66 & -13.3 & 4.5 \\
\hline 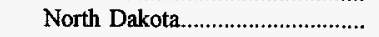 & - & - & 12 & 82 & - & - & 208 & 80 & - & - \\
\hline 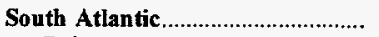 & 11,125 & 141 & 9,720 & 145 & 22,494 & 142 & 19,631 & 147 & 14.6 & -3.4 \\
\hline Delaware & 12 & 165 & 79 & 147 & 16 & 162 & 189 & 153 & -91.8 & 6.1 \\
\hline 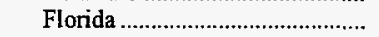 & 2,364 & 151 & 2,540 & 157 & 4,674 & 153 & 4,800 & 162 & -2.6 & -5.5 \\
\hline Georgia & 3,719 & 149 & 3,387 & 149 & 7,509 & 149 & 6,059 & 148 & 23.9 & 9 \\
\hline 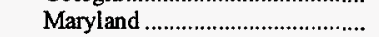 & 701 & 146 & 399 & 165 & 1,618 & 146 & 1,149 & 164 & 40.8 & -11.0 \\
\hline 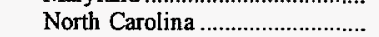 & 1,352 & 130 & 1,567 & 129 & 3,445 & 133 & 3,458 & 135 & -.4 & -1.9 \\
\hline 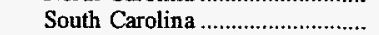 & 711 & 145 & 765 & 142 & 1,238 & 146 & 1,437 & 143 & -13.9 & 1.8 \\
\hline 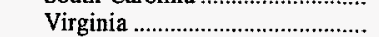 & 1,116 & 135 & 586 & 139 & 1,837 & 136 & 1,364 & 140 & 34.7 & -3.1 \\
\hline 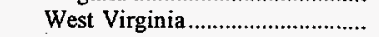 & 1,150 & 107 & 398 & 107 & 2,159 & 107 & 1,174 & 107 & 83.8 & $*$ \\
\hline 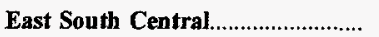 & 5,612 & 113 & 6,517 & 112 & 12,798 & 113 & 12,138 & 114 & 5.4 & -.5 \\
\hline Alabama & 952 & 127 & 1,278 & 125 & 2,185 & 128 & 2,636 & 125 & -17.1 & 2.6 \\
\hline 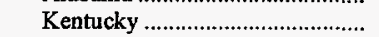 & 3,370 & 105 & 3,190 & 104 & 7,011 & 105 & 5,519 & 105 & 27.0 & -.2 \\
\hline 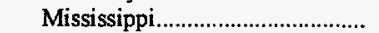 & 297 & 136 & 190 & 144 & 417 & 137 & 340 & 142 & 22.7 & -3.3 \\
\hline 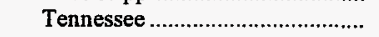 & 992 & 118 & 1,859 & 113 & 3,185 & 117 & 3,644 & 116 & -12.6 & .9 \\
\hline West South Central.............................. & 2,134 & 123 & 1,602 & 125 & 4,211 & 122 & 3,200 & 124 & 31.6 & -1.8 \\
\hline 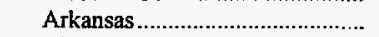 & 214 & 118 & 162 & 128 & 687 & 100 & 398 & 118 & 72.6 & -15.0 \\
\hline 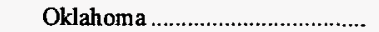 & 142 & 92 & 12 & 84 & 142 & 92 & 12 & 84 & NM & 9.0 \\
\hline Texas & 1,778 & 126 & 1,428 & 125 & 3,382 & 127 & 2,791 & 125 & 21.2 & 1.8 \\
\hline Mountain & 1,454 & 87 & 1,453 & 105 & 3,237 & 90 & 3,145 & 101 & 2.9 & -10.8 \\
\hline Arizona & 683 & 107 & 614 & 120 & 1,282 & 107 & 1,190 & 121 & 7.7 & -11.6 \\
\hline 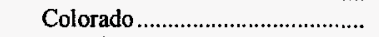 & 290 & 72 & 294 & 90 & 598 & 76 & 760 & 86 & -21.3 & -11.2 \\
\hline 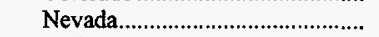 & - & - & 86 & 126 & 277 & 122 & 180 & 126 & 54.1 & -3.8 \\
\hline Utah & 85 & 99 & 353 & 86 & 142 & 99 & 553 & 90 & -74.4 & 9.9 \\
\hline 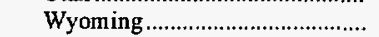 & 396 & 63 & 106 & 106 & 938 & 63 & 461 & 77 & 103.5 & -18.8 \\
\hline 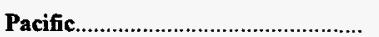 & 815 & 117 & - & - & 1,713 & 115 & 139 & 116 & $\mathbf{N M}$ & -1.4 \\
\hline Oregon & 304 & 110 & - & - & 965 & 109 & 135 & 114 & NM & -4.4 \\
\hline 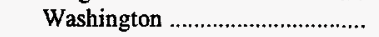 & 511 & 121 & - & - & 748 & 121 & 4 & 172 & NM & -29.6 \\
\hline 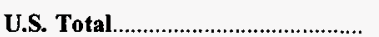 & 41,373 & 120 & 36,868 & 121 & 84,678 & 120 & 73,641 & 122 & 15.0 & -2.1 \\
\hline
\end{tabular}

* For percentage calculations, the absolute value of the number is less than 0.05 percent.

NM Percent change calculation not meaningful as value is greater than 500 .

Notes: Total may not equal sum of components because of independent rounding. MM Btu represents million Btu.

Source: Federal Energy Regulatory Commission, FERC Form 423, "Monthly Report of Cost and Quality of Fuels for Electric Plants." 
Table 26. Average Cost of Coal Receipts at Electric Utility Plants by Census Division and State (Dollars per Short Ton)

\begin{tabular}{|c|c|c|c|c|c|c|}
\hline \multirow{2}{*}{$\begin{array}{c}\text { Census Division } \\
\text { and State }\end{array}$} & \multirow{2}{*}{$\begin{array}{c}\text { April-June } \\
1998\end{array}$} & \multirow{2}{*}{$\begin{array}{c}\text { January-March } \\
1998\end{array}$} & \multirow{2}{*}{$\begin{array}{c}\text { April-June } \\
1997\end{array}$} & \multicolumn{3}{|c|}{ Year to Date } \\
\hline & & & & 1998 & 1997 & Percent Change \\
\hline 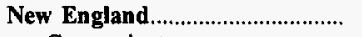 & $\$ 42.31$ & $\$ 43.44$ & $\$ 43.56$ & $\$ 42.92$ & $\$ 44.01$ & -2.5 \\
\hline 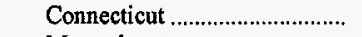 & 46.72 & 48.28 & 50.58 & 47.68 & 50.47 & -5.5 \\
\hline 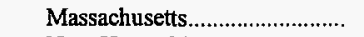 & 41.79 & 42.66 & 42.06 & 42.24 & 43.09 & -2.0 \\
\hline 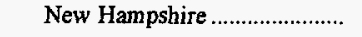 & 41.80 & 42.55 & 42.12 & 42.23 & 42.17 & .1 \\
\hline Mid Atlantic & 34.44 & 34.64 & 33.86 & 34.54 & 34.62 & -.2 \\
\hline 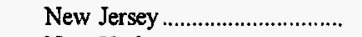 & 41.35 & 43.62 & 46.39 & 42.32 & 46.18 & -8.4 \\
\hline New York & 37.19 & 38.30 & 37.56 & 37.70 & 36.96 & 2.0 \\
\hline Pennsylvania ................................... & 33.50 & 33.66 & 32.63 & 33.58 & 33.64 & -.2 \\
\hline East North Central.......................... & 27.67 & 27.59 & 27.61 & 27.63 & 28.11 & -1.7 \\
\hline 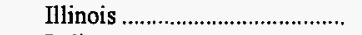 & 31.99 & 30.35 & 30.58 & 31.15 & 32.29 & -3.5 \\
\hline 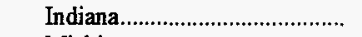 & 23.39 & 23.77 & 23.96 & 23.58 & 24.24 & -2.7 \\
\hline Michigan ................................. & 27.56 & 28.61 & 29.17 & 27.96 & 29.22 & -4.3 \\
\hline Ohio & 32.45 & 32.58 & 31.32 & 32.51 & 31.50 & 3.2 \\
\hline 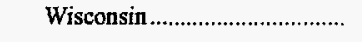 & 20.71 & 18.82 & 21.05 & 19.85 & 20.24 & -1.9 \\
\hline West North Central ........................ & 15.36 & 14.95 & 15.56 & 15.15 & 15.50 & -2.3 \\
\hline 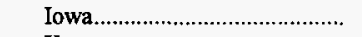 & 15.91 & 15.03 & 16.34 & 15.50 & 15.94 & -2.7 \\
\hline 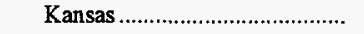 & 17.33 & 17.16 & 19.60 & 17.24 & 18.67 & -7.7 \\
\hline Minnesota .................................. & 19.57 & 19.67 & 20.06 & 19.63 & 19.95 & -1.6 \\
\hline Missouri........................................ & 16.51 & 16.36 & 16.90 & 16.44 & 16.92 & -2.8 \\
\hline 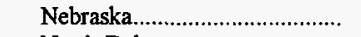 & 10.10 & 10.06 & 10.41 & 10.08 & 10.25 & -1.7 \\
\hline 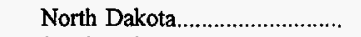 & 10.57 & 9.81 & 9.85 & 10.15 & 10.08 & .7 \\
\hline 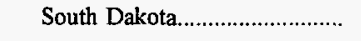 & 16.10 & 16.25 & 15.94 & 16.17 & 16.10 & .4 \\
\hline South Atlantic............................. & 35.56 & 35.73 & 36.41 & 35.65 & 36.49 & -2.3 \\
\hline 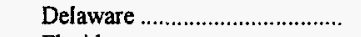 & 41.10 & 40.05 & 41.25 & 40.52 & 41.90 & -3.3 \\
\hline Florida & 39.90 & 41.15 & 41.84 & 40.51 & 42.48 & -4.6 \\
\hline 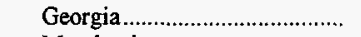 & 36.44 & 36.41 & 37.85 & 36.42 & 37.30 & -2.3 \\
\hline 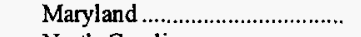 & 37.64 & 37.61 & 38.84 & 37.62 & 39.34 & -4.4 \\
\hline North Carolina .............................. & 35.84 & 35.41 & 35.00 & 35.62 & 35.45 & .5 \\
\hline South Carolina .......................... & 36.97 & 37.06 & 36.98 & 37.01 & 37.33 & -.9 \\
\hline 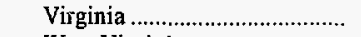 & 34.71 & 34.94 & 34.77 & 34.82 & 34.92 & -.3 \\
\hline West Virginia ................................. & 29.96 & 30.11 & 30.69 & 30.04 & 30.69 & -2.1 \\
\hline East South Central......................... & 28.85 & 28.69 & 28.63 & 28.77 & 28.87 & -.3 \\
\hline 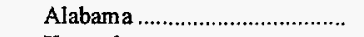 & 36.37 & 35.53 & 35.66 & 35.95 & 35.71 & .7 \\
\hline 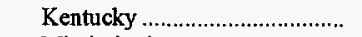 & 24.23 & 24.35 & 23.93 & 24.29 & 24.08 & .8 \\
\hline Mississippi............................... & 31.96 & 31.95 & 32.66 & 31.96 & 32.20 & -.8 \\
\hline 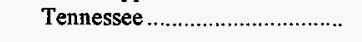 & 26.26 & 26.70 & 26.68 & 26.50 & 26.99 & -1.8 \\
\hline West South Central.......................... & 19.65 & 20.15 & 20.28 & 19.89 & 20.02 & -.6 \\
\hline 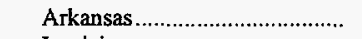 & 25.97 & 25.80 & 29.69 & 25.89 & 29.27 & -11.5 \\
\hline 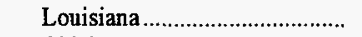 & 22.91 & 23.08 & 24.14 & 23.00 & 24.30 & -5.4 \\
\hline Oklahoma & 15.98 & 16.04 & 16.13 & 16.01 & 16.07 & -.4 \\
\hline 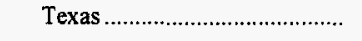 & 19.07 & 19.88 & 19.37 & 19.46 & 19.01 & 2.3 \\
\hline 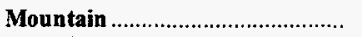 & 21.31 & 20.84 & 22.21 & 21.07 & 22.02 & -4.3 \\
\hline 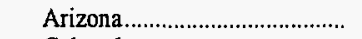 & 27.52 & 27.05 & 29.64 & 27.29 & 29.82 & -8.5 \\
\hline 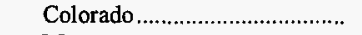 & 20.05 & 19.53 & 20.59 & 19.79 & 20.69 & -4.3 \\
\hline Montana.................................... & 12.84 & 11.39 & 11.62 & 12.06 & 11.45 & 5.4 \\
\hline 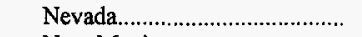 & 34.09 & 29.23 & 34.04 & 31.15 & 31.60 & -1.4 \\
\hline New Mexico................................... & 25.03 & 23.68 & 24.16 & 24.36 & 24.54 & -.7 \\
\hline Utah & 26.54 & 25.66 & 25.08 & 26.10 & 25.63 & 1.8 \\
\hline 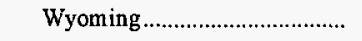 & 12.35 & 14.07 & 13.84 & 13.23 & 14.17 & -6.6 \\
\hline 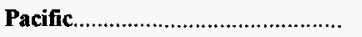 & 22.93 & 24.00 & 30.53 & 23.43 & 30.39 & -22.9 \\
\hline Oregon & 19.09 & 19.15 & - & 19.13 & 20.00 & -4.3 \\
\hline 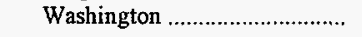 & 23.61 & 26.85 & 30.53 & 24.90 & 31.14 & -20.0 \\
\hline 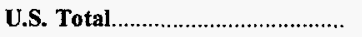 & 25.90 & 25.85 & 26.49 & 25.88 & 26.51 & -2.4 \\
\hline
\end{tabular}

Notes: Total may not equal sum of components because of independent rounding

Source: Federal Energy Regulatory Commission, FERC Form 423, "Monthly Report of Cost and Quality of Fuels for Electric Plants." 
Table 27. Coal Receipts and Prices by Sulfur Content at Electric Utility Plants, by State of Origin and Imports, January-June 1998

\begin{tabular}{|c|c|c|c|c|c|c|c|c|c|c|c|c|}
\hline \multirow[b]{2}{*}{ State } & \multicolumn{2}{|c|}{$\begin{array}{c}\text { 0-0.60 lbs } \\
\text { sulfur } \\
\text { per MM Btu }\end{array}$} & \multicolumn{2}{|c|}{$\begin{array}{c}\text { 0.61-1.67 lbs } \\
\text { sulfur } \\
\text { per MM Btu }\end{array}$} & \multicolumn{2}{|c|}{$\begin{array}{c}>\quad 1.67 \mathrm{lbs} . \\
\text { sulfur } \\
\text { per MM Btu }\end{array}$} & \multicolumn{3}{|c|}{ Total } & \multicolumn{3}{|c|}{$\begin{array}{c}\text { Percent Change vs } \\
\text { prior year }\end{array}$} \\
\hline & $\begin{array}{c}\text { Quantity } \\
\text { (thousand } \\
\text { short } \\
\text { tons) }\end{array}$ & $\begin{array}{c}\text { Price } \\
\text { (cents } \\
\text { per } \\
\text { MM } \\
\text { Btu) }\end{array}$ & $\begin{array}{c}\text { Quantity } \\
\text { (thousand } \\
\text { short } \\
\text { tons) }\end{array}$ & $\begin{array}{c}\text { Price } \\
\text { (cents } \\
\text { per } \\
\text { MM } \\
\text { Btu) }\end{array}$ & $\begin{array}{l}\text { Quantity } \\
\text { (thousand } \\
\text { short } \\
\text { tons) }\end{array}$ & $\begin{array}{c}\text { Price } \\
\text { (cents } \\
\text { per } \\
\text { MM } \\
\text { Btu) }\end{array}$ & $\begin{array}{c}\text { Quantity } \\
\text { (thousand } \\
\text { short } \\
\text { tons) }\end{array}$ & $\begin{array}{c}\text { Price } \\
\text { (cents } \\
\text { per } \\
\text { MM } \\
\text { Btu) }\end{array}$ & $\begin{array}{c}\text { Lbs. } \\
\text { sulfur } \\
\text { per } \\
\text { MM Btu }\end{array}$ & Quantity & Price & $\begin{array}{c}\text { Sulfur } \\
\text { Content }\end{array}$ \\
\hline 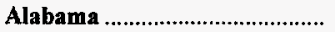 & 3,806 & 205 & 3,101 & 166 & 729 & 136 & 7,636 & 183 & 0.95 & -10.3 & 2.6 & 3.7 \\
\hline Arizona & 5,839 & 120 & - & - & - & - & 5,839 & 120 & .49 & 17.9 & -7.0 & 2.0 \\
\hline Colorado & 10,732 & 123 & 165 & 94 & - & - & 10,897 & 123 & .41 & -5.2 & -1.3 & $*$ \\
\hline 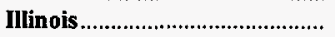 & 28 & 126 & 7,201 & 141 & 10,172 & 129 & 17,400 & 134 & 2.00 & -2.3 & -2.0 & -1.8 \\
\hline Indiana & 876 & 149 & 5,755 & 121 & 9,573 & 101 & 16,205 & 110 & 2.07 & 16.2 & 2.8 & -2.4 \\
\hline 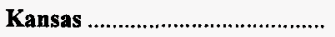 & - & - & - & - & 202 & 103 & 202 & 103 & 3.86 & 65.5 & -14.7 & 25.7 \\
\hline 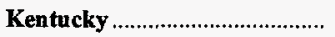 & 8,317 & 153 & 36,252 & 141 & 15,709 & 107 & 60,278 & 134 & 1.33 & 1.7 & -1.4 & -3.3 \\
\hline 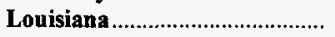 & - & - & 855 & 129 & 696 & 148 & 1,551 & 137 & 1.47 & -14.6 & 2.6 & -4.5 \\
\hline 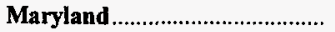 & - & - & 2,056 & 122 & - & - & 2,056 & 122 & 1.37 & 17.3 & -1.9 & 2.6 \\
\hline 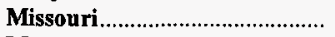 & - & - & - & - & 75 & 121 & 75 & 121 & 2.81 & -62.9 & 13.5 & -19.1 \\
\hline 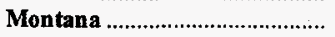 & 8,345 & 144 & 9,797 & 90 & - & - & 18,142 & 116 & .61 & 12.2 & -9.0 & 9 \\
\hline 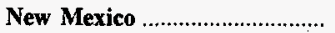 & 3,265 & 153 & 9,408 & 134 & - & - & 12,673 & 140 & .74 & -2.9 & -4.2 & -.8 \\
\hline 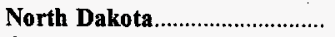 & - & - & 10,887 & 77 & 537 & 80 & 11,423 & 77 & 1.16 & 3.0 & 1.3 & .2 \\
\hline 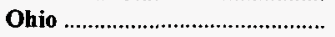 & 2 & 154 & 195 & 125 & 11,822 & 140 & 12,019 & 140 & 3.00 & -6.0 & 9.0 & 2.2 \\
\hline 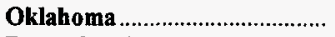 & - & - & - & - & 68 & 112 & 68 & 112 & 2.85 & 36.5 & 5.9 & 7.2 \\
\hline Pennsylvania & 876 & 150 & 20,969 & 135 & 6,448 & 119 & 28,293 & 132 & 1.45 & 12.9 & .5 & -.9 \\
\hline 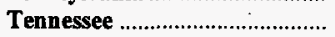 & - & - & 1,255 & 127 & - & - & 1,255 & 127 & 1.03 & -1.2 & * & 13.0 \\
\hline 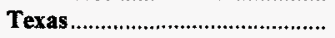 & - & - & 11,094 & 120 & 12,109 & 89 & 23,203 & 104 & 1.67 & -2.4 & 4.0 & 7.2 \\
\hline 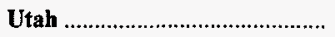 & 9,074 & 120 & 268 & 124 & - & - & 9,342 & 120 & .41 & -3.1 & 2.1 & -2.0 \\
\hline 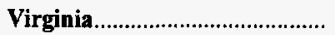 & 3,111 & 155 & 4,815 & 139 & 138 & 125 & 8,063 & 145 & .76 & 9.2 & -1.3 & -9.9 \\
\hline 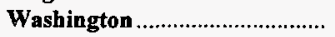 & - & - & 2,080 & 164 & - & - & 2,080 & 164 & .79 & 19.1 & -17.4 & 3.3 \\
\hline 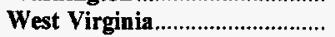 & 17,160 & 151 & 22,484 & 136 & 13,179 & 133 & 52,823 & 140 & 1.24 & .5 & -.9 & 3.4 \\
\hline Wyoming & 139,039 & 112 & 9,111 & 105 & - & - & 148,150 & 112 & .39 & 11.9 & -4.8 & -2.7 \\
\hline 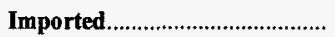 & 2,546 & 157 & 130 & 150 & - & - & 2,676 & 157 & .52 & 43.6 & -1.3 & -10.5 \\
\hline 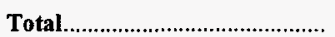 & 213,016 & 126 & 157,876 & 131 & 81,457 & 119 & 452,348 & 126 & 1.06 & 5.5 & -2.0 & -2.1 \\
\hline
\end{tabular}

* For percentage calculations, the absolute value of the number is less than 0.05 percent.

Notes: Total may not equal sum of components because of independent rounding. MM Btu represents million Btu.

Source: Federal Energy Regulatory Commission, FERC Form 423, "Monthly Report of Cast and Quality of Fuels for Electric Plants." 
Table 28. Destination of Coal Received at Electric Utility Plants by Origin, January-June 1998, 1997

\begin{tabular}{|c|c|c|c|c|c|c|c|c|}
\hline \multirow{2}{*}{$\begin{array}{l}\text { State of Destination } \\
\text { State of Origin } \\
\text { and Imports }\end{array}$} & \multicolumn{2}{|c|}{$\begin{array}{l}\text { Receipts } \\
\text { (thousand short tons) }\end{array}$} & \multicolumn{2}{|c|}{$\begin{array}{c}\text { Contract Receipts } \\
\text { (percent) }\end{array}$} & \multicolumn{2}{|c|}{$\begin{array}{l}\text { Sulfur Content } \\
\text { (lbs. sulfur } \\
\text { per MM Btu) }\end{array}$} & \multicolumn{2}{|c|}{$\begin{array}{c}\text { Price } \\
\text { (cents per MM Btu) }\end{array}$} \\
\hline & 1998 & 1997 & 1998 & 1997 & 1998 & 1997 & 1998 & 1997 \\
\hline Alabama & 14,678 & 15,535 & 85.1 & 83.0 & 1.03 & 0.97 & 156 & 153 \\
\hline 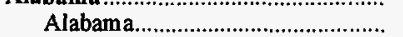 & 7,499 & 8,228 & 93.4 & 92.4 & .95 & .89 & 183 & 179 \\
\hline Colorado & 340 & 653 & 100.0 & 100.0 & .42 & .44 & 116 & 113 \\
\hline 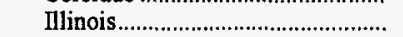 & 435 & 854 & 14.0 & - & 1.72 & 1.20 & 128 & 130 \\
\hline Indiana & 49 & - & - & - & 3.12 & - & 110 & - \\
\hline 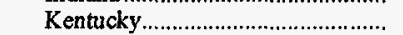 & 2,121 & 1,344 & 65.1 & 66.5 & 1.65 & 1.95 & 120 & 112 \\
\hline Pennsylvania & 29 & 46 & 100.0 & 100.0 & 1.53 & 1.80 & 112 & 112 \\
\hline Tennessee & 321 & 284 & 100.0 & 100.0 & .70 & .71 & 136 & 134 \\
\hline Utah & - & 122 & - & 81.1 & - & .39 & - & 122 \\
\hline West Virginia & 1,061 & 1,797 & 51.7 & 63.4 & 1.57 & 1.36 & 132 & 129 \\
\hline Wyoming & 2,823 & 2,206 & 99.5 & 98.9 & .31 & .29 & 119 & 112 \\
\hline 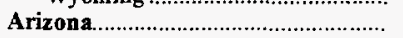 & 9,104 & 7,680 & 85.9 & 84.5 & .54 &. $\mathbf{5 3}$ & 134 & 147 \\
\hline Arizona & 3,711 & 2,819 & 100.0 & 100.0 & .49 & .49 & 118 & 125 \\
\hline 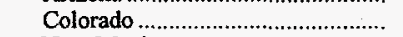 & 30 & - & - & - & .40 & - & 154 & - \\
\hline 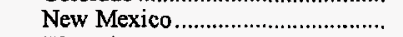 & 5,167 & 4,860 & 79.6 & 75.5 & .58 & .55 & 147 & 161 \\
\hline 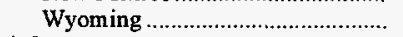 & 195 & - & - & - & .29 & - & 117 & - \\
\hline 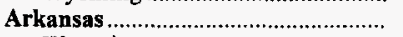 & 6,580 & 5,865 & 89.6 & 93.2 & .38 & .38 & 149 & 169 \\
\hline 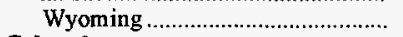 & 6,580 & 5,865 & 89.6 & 93.2 & .38 & .38 & 149 & 169 \\
\hline Colorado & 8,843 & 8,140 & 93.2 & 90.7 & .39 & .38 & 100 & 104 \\
\hline 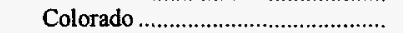 & 5,488 & 5,299 & 93.1 & 90.1 & .41 & .41 & 106 & 111 \\
\hline Wyoming & 3,355 & 2,841 & 93.4 & 91.6 & .34 & .32 & 88 & 89 \\
\hline Connecticut & 452 & 548 & 79.2 & 94.0 & .40 & .43 & 181 & 192 \\
\hline 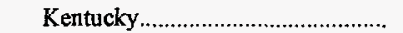 & 352 & 405 & 87.8 & 98.8 & .38 & .41 & 183 & 193 \\
\hline Virginia & - & 4 & - & 100.0 & - & .52 & - & 184 \\
\hline 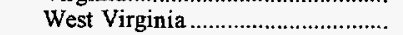 & 64 & 139 & 76.6 & 79.9 & .46 & .48 & 181 & 190 \\
\hline Imported coal Venezuela ................. & 36 & - & - & - & .42 & - & 162 & - \\
\hline Delaware & 730 & 868 & 97.9 & 78.2 & .76 & .70 & 157 & 161 \\
\hline Kentucky & 54 & - & 100.0 & - & .50 & - & 176 & - \\
\hline 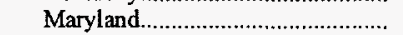 & 57 & 39 & 100.0 & 100.0 & 1.13 & 1.09 & 146 & 149 \\
\hline 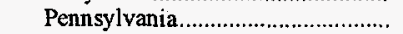 & 240 & 217 & 97.7 & 59.4 & 1.04 & 1.02 & 143 & 142 \\
\hline Virginia & 65 & 76 & 100.0 & 88.3 & .61 & .67 & 175 & 158 \\
\hline West Virginia & 315 & 537 & 96.8 & 82.8 & .55 & .55 & 163 & 170 \\
\hline Florida & 13,839 & $\mathbf{1 3 , 5 3 7}$ & 66.2 & 64.5 & 1.29 & 1.33 & 167 & 175 \\
\hline 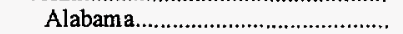 & 109 & 193 & - & - & .88 & 1.94 & 162 & 176 \\
\hline Colorado & - & 14 & - & - & - & .54 & - & 183 \\
\hline 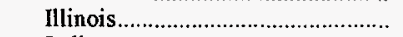 & 3,529 & 3,035 & 80.9 & 77.7 & 1.86 & 1.87 & 167 & 184 \\
\hline Indiana & 30 & 5 & 100.0 & - & 2.79 & .62 & 125 & 164 \\
\hline 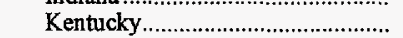 & 7,078 & 7,113 & 64.3 & 62.2 & 1.21 & 1.30 & 168 & 175 \\
\hline Virginia & 407 & 454 & 97.8 & 96.0 & .63 & .67 & 209 & 210 \\
\hline 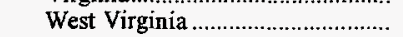 & 1,018 & 1,351 & 44.0 & 49.2 & 1.36 & 1.17 & 164 & 168 \\
\hline 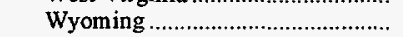 & 478 & 520 & - & - & .46 & .51 & 136 & 143 \\
\hline Imported coal Colombia ................... & 700 & 565 & 93.8 & 100.0 & .53 & .60 & 148 & 152 \\
\hline 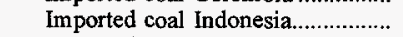 & 297 & 287 & 75.4 & 100.0 & .26 & .38 & 159 & 162 \\
\hline Imported coal Venezuela .............. & 193 & - & - & - & .60 & - & 156 & - \\
\hline 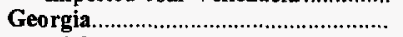 & 14,804 & 13,657 & 49.3 & 55.6 & .72 & .70 & 155 & 159 \\
\hline 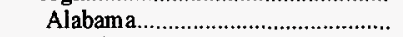 & - & 50 & - & - & - & 1.51 & - & 135 \\
\hline 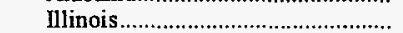 & 400 & 450 & 100.0 & 89.6 & 1.27 & .87 & 137 & 147 \\
\hline 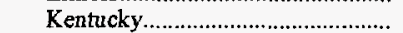 & 7,249 & 7,294 & 54.9 & 65.6 & .82 & .77 & 150 & 155 \\
\hline 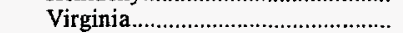 & 1,856 & 963 & 60.0 & 75.7 & .68 & .73 & 153 & 161 \\
\hline West Virginia & 2,358 & 2,162 & 76.5 & 77.8 & .60 & .57 & 182 & 184 \\
\hline Wyoming & 2,790 & 2,640 & - & - & .41 & .45 & 150 & 150 \\
\hline Imported coal Venezuela ................ & 150 & 99 & - & - & .88 & 1.34 & 150 & 138 \\
\hline 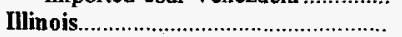 & 19,467 & 21,043 & 87.7 & 83.8 & 1.15 & 1.21 & 160 & 164 \\
\hline Colorado & 690 & 611 & 83.2 & 55.0 & .45 & .47 & 138 & 136 \\
\hline 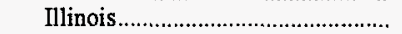 & 6,920 & 7,374 & 88.1 & 82.8 & 2.33 & 2.49 & 127 & 124 \\
\hline Indiana & 836 & 825 & 70.6 & 49.9 & .84 & .95 & 147 & 140 \\
\hline Kentucky & 9 & 88 & - & 20.5 & .57 & .45 & 169 & 172 \\
\hline Montana & 655 & 790 & 100.0 & 100.0 & .34 & .33 & 234 & 262 \\
\hline Utah & 434 & 880 & 90.3 & 59.8 & .37 & .38 & 179 & 171 \\
\hline West Virginia & - & 7 & - & - & - & .57 & - & 146 \\
\hline Wyoming & 9,923 & 10,469 & 88.3 & 90.3 & .33 & .38 & 186 & 194 \\
\hline Indiana & 28,128 & 25,817 & $\mathbf{7 1 . 2}$ & 71.7 & 1.55 & 1.54 & 113 & 116 \\
\hline 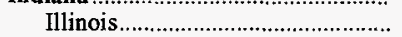 & 2,338 & 2,789 & 65.0 & 89.6 & 2.17 & 1.99 & 119 & 131 \\
\hline Indiana & 13,879 & 11,646 & 66.5 & 57.1 & 2.10 & 2.15 & 109 & 106 \\
\hline 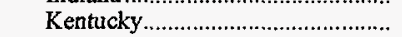 & 668 & 454 & 56.4 & 84.1 & 1.37 & 1.36 & 122 & 125 \\
\hline Montana & - & 604 & - & 100.0 & - & .33 & - & 253 \\
\hline Ohio & 562 & 613 & - & - & 3.38 & 3.57 & 109 & 101 \\
\hline 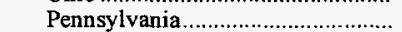 & 283 & 264 & 100.0 & 98.7 & 1.67 & 1.75 & 107 & 104 \\
\hline 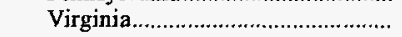 & 443 & 426 & 100.0 & 100.0 & .54 & .54 & 159 & 157 \\
\hline
\end{tabular}

See footnotes at end of table. 
Table 28. Destination of Coal Received at Electric Utility Plants by Origin, January-June 1998, 1997 (Continued)

\begin{tabular}{|c|c|c|c|c|c|c|c|c|}
\hline \multirow{2}{*}{$\begin{array}{l}\text { State of Destination } \\
\text { State of Origin } \\
\text { and Imports }\end{array}$} & \multicolumn{2}{|c|}{$\begin{array}{c}\text { Receipts } \\
\text { (thousand short tons) }\end{array}$} & \multicolumn{2}{|c|}{$\begin{array}{c}\text { Contract Receipts } \\
\text { (percent) }\end{array}$} & \multicolumn{2}{|c|}{$\begin{array}{c}\text { Sulfur Content } \\
\text { (lbs. sulfur } \\
\text { per MM Btu) }\end{array}$} & \multicolumn{2}{|c|}{$\begin{array}{c}\text { Price } \\
\text { (cents per MM Btu) }\end{array}$} \\
\hline & 1998 & 1997 & 1998 & 1997 & 1998 & 1997 & 1998 & 1997 \\
\hline \multicolumn{9}{|l|}{ Indiana } \\
\hline 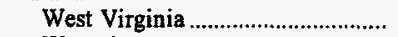 & 900 & 538 & 53.7 & 57.8 & 0.86 & 0.95 & 119 & 142 \\
\hline 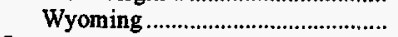 & 9,054 & 8,483 & 85.1 & 87.1 & .34 & .34 & 113 & 113 \\
\hline Iowa & 10,070 & 8,378 & 76.4 & 81.6 & .49 & .47 & 90 & 92 \\
\hline 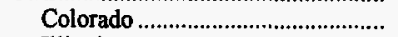 & 249 & 260 & 100.0 & 88.4 & .43 & .46 & 132 & 135 \\
\hline 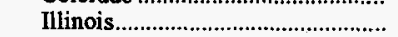 & 102 & 47 & 28.0 & 72.1 & 2.32 & 2.13 & 117 & 128 \\
\hline Indiana & 90 & 66 & 100.0 & 24.5 & 1.23 & 1.28 & 125 & 123 \\
\hline 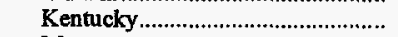 & 55 & 31 & 59.8 & - & 2.35 & 2.30 & 117 & 112 \\
\hline Montana & 50 & 29 & 44.6 & - & .30 & .30 & 135 & 148 \\
\hline 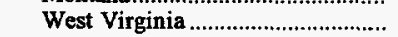 & 1 & 1 & - & - & 2.08 & 2.08 & 164 & 153 \\
\hline 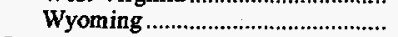 & 9,523 & 7,944 & 76.3 & 82.5 & .44 & .44 & 87 & 90 \\
\hline 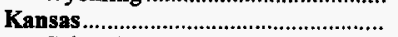 & 9,398 & 7,963 & 99.2 & 99.2 & .52 & .57 & 99 & 106 \\
\hline Colorado & 603 & 842 & 100.0 & 100.0 & .42 & .37 & 122 & 125 \\
\hline 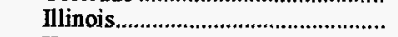 & - & 88 & - & 100.0 & - & 2.69 & - & 107 \\
\hline 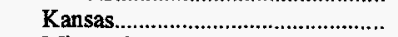 & 202 & 84 & 100.0 & 100.0 & 3.86 & 3.17 & 103 & 115 \\
\hline 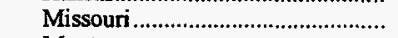 & 14 & 152 & 100.0 & 100.0 & 3.06 & 3.79 & 126 & 101 \\
\hline 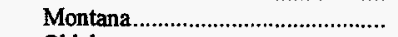 & 67 & - & 100.0 & - & .34 & - & 91 & - \\
\hline 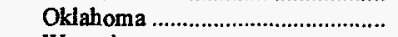 & 38 & 8 & 100.0 & - & 2.90 & 2.91 & 118 & 120 \\
\hline 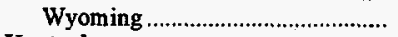 & 8,474 & 6,789 & 99.1 & 99.2 & .40 & .43 & 96 & 103 \\
\hline 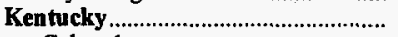 & 19,105 & 19,308 & 63.3 & 71.4 & 2.10 & 2.19 & 105 & 104 \\
\hline 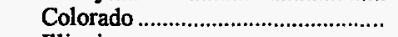 & 1,302 & 993 & 100.0 & 100.0 & .40 & .43 & 126 & 129 \\
\hline 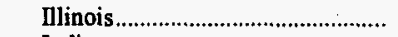 & 143 & 91 & - & - & 2.04 & 2.35 & 100 & 104 \\
\hline Indiana & 1,074 & 1,222 & 93.9 & 99.3 & 2.88 & 2.82 & 95 & 91 \\
\hline 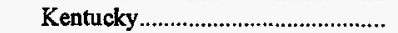 & 12,969 & 12,704 & 55.0 & 68.8 & 2.37 & 2.53 & 103 & 101 \\
\hline 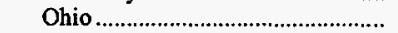 & 357 & 316 & 87.9 & 41.7 & 3.40 & 3.68 & 96 & 89 \\
\hline 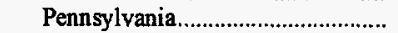 & 48 & 57 & 80.7 & 64.0 & 1.72 & 1.65 & 110 & 111 \\
\hline Utah & - & 132 & - & 92.4 & - & .54 & - & 134 \\
\hline West Virginia & 3,118 & 3,582 & 72.8 & 66.5 & 1.41 & 1.32 & 111 & 112 \\
\hline Wyoming & 94 & 212 & 23.9 & 79.6 & .32 & .56 & 94 & 119 \\
\hline 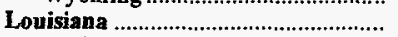 & 6,451 & 6,506 & 100.0 & 100.0 & .74 & .78 & 142 & 150 \\
\hline Louisiana & 1,551 & 1,817 & 100.0 & 100.0 & 1.47 & 1.54 & 137 & 134 \\
\hline 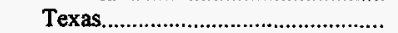 & 91 & - & 100.0 & - & 2.31 & - & 132 & - \\
\hline 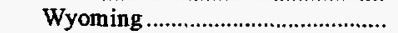 & 4,809 & 4,689 & 100.0 & 100.0 & .52 & .54 & 144 & 155 \\
\hline 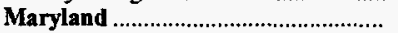 & 5,490 & 4,896 & $\mathbf{7 0 . 5}$ & 76.5 & .91 & .86 & 146 & 152 \\
\hline 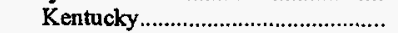 & 259 & 269 & 100.0 & 85.9 & .58 & .58 & 146 & 151 \\
\hline 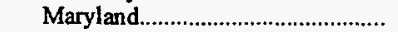 & 343 & 393 & 84.2 & 85.0 & 1.15 & 1.09 & 175 & 173 \\
\hline 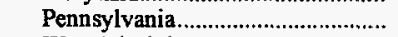 & 1,516 & 554 & 16.4 & 61.2 & 1.13 & 1.06 & 149 & 157 \\
\hline 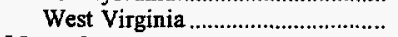 & 3,372 & 3,680 & 91.2 & 77.3 & .80 & .82 & 142 & 150 \\
\hline 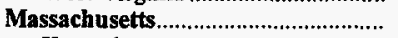 & 2,482 & 2,243 & 87.6 & 90.0 & .57 & .58 & 168 & 171 \\
\hline 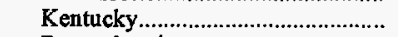 & 388 & 405 & 97.9 & 91.7 & .53 & .55 & 181 & 181 \\
\hline 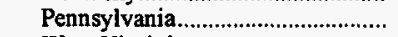 & 87 & 125 & 100.0 & 73.0 & 1.19 & .99 & 161 & 166 \\
\hline West Virginia & 1,313 & 1,091 & 77.1 & 85.6 & .56 & .56 & 168 & 173 \\
\hline Imported coal Colombia ................ & 387 & 511 & 100.0 & 100.0 & .51 & .53 & 166 & 163 \\
\hline Imported coal Venezuela .............. & 307 & 112 & 100.0 & 100.0 & .51 & .56 & 153 & 163 \\
\hline 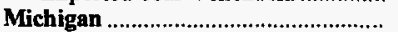 & 15,875 & 13,846 & 76.6 & 82.8 & .66 & .64 & 132 & 137 \\
\hline Colorado & 288 & 182 & 94.0 & 94.4 & .48 & .46 & 138 & 135 \\
\hline Illinois & 9 & - & - & - & 1.08 & - & 152 & - \\
\hline Indiana & 106 & 69 & 100.0 & 100.0 & 2.11 & 2.01 & 138 & 136 \\
\hline 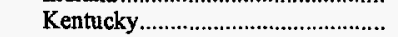 & 2,173 & 1,993 & 64.2 & 63.8 & .68 & .72 & 150 & 154 \\
\hline 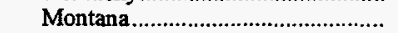 & 3,866 & 3,025 & 89.2 & 100.0 & .39 & .39 & 143 & 157 \\
\hline Ohio & 74 & 52 & 18.8 & 97.6 & 2.66 & 2.50 & 157 & 155 \\
\hline 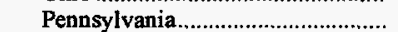 & 1,737 & 1,213 & 64.8 & 84.3 & 1.16 & 1.18 & 125 & 121 \\
\hline 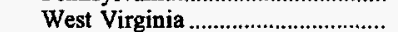 & 2,475 & 2,579 & 54.5 & 71.9 & .93 & .88 & 152 & 155 \\
\hline Wyoming & 5,145 & 4,733 & 86.5 & 84.6 & .35 & .33 & 99 & 107 \\
\hline Minnesota & 8,567 & 8,275 & 97.3 & 96.8 & .49 & .49 & 111 & 112 \\
\hline Illinois & 11 & 52 & 100.0 & 100.0 & 1.10 & 1.12 & 161 & 166 \\
\hline Indiana & 15 & - & - & - & 1.13 & - & 150 & - \\
\hline 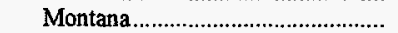 & 4,526 & 4,320 & 96.1 & 93.8 & .68 & .66 & 111 & 112 \\
\hline 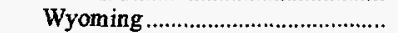 & 4,014 & 3,902 & 99.0 & 100.0 & .28 & .29 & 110 & 112 \\
\hline 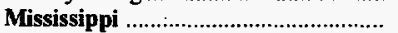 & 3,058 & 2,893 & 86.4 & 88.3 & .74 & .67 & 153 & 154 \\
\hline 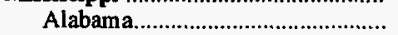 & 28 & 46 & - & - & .57 & .63 & 148 & 138 \\
\hline Colorado & - & 36 & - & - & - & .42 & - & 155 \\
\hline 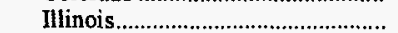 & 744 & 626 & 89.6 & 87.9 & 1.39 & 1.02 & 142 & 138 \\
\hline 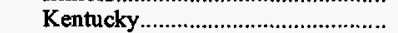 & 384 & 541 & 96.0 & 87.7 & .76 & .80 & 203 & 198 \\
\hline Montana & 1,606 & 1,528 & 100.0 & 100.0 & .40 & .43 & 147 & 143 \\
\hline 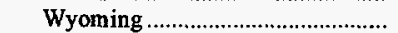 & 231 & 116 & - & - & .48 & .53 & 133 & 136 \\
\hline 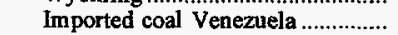 & 65 & - & - & - & .60 & - & 141 & - \\
\hline 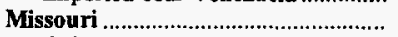 & 18,968 & 15,910 & 61.9 & 85.0 & .42 & .53 & 92 & 94 \\
\hline 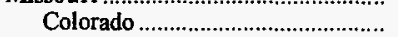 & 30 & - & - & - & .54 & - & 137 & - \\
\hline
\end{tabular}

See footnotes at end of table 
Table 28. Destination of Coal Received at Electric Utility Plants by Origin, January-June 1998, 1997 (Continued)

\begin{tabular}{|c|c|c|c|c|c|c|c|c|}
\hline \multirow{2}{*}{$\begin{array}{l}\text { State of Destination } \\
\text { State of Origin } \\
\text { and Imports }\end{array}$} & \multicolumn{2}{|c|}{$\begin{array}{c}\text { Receipts } \\
\text { (thousand short tons) }\end{array}$} & \multicolumn{2}{|c|}{$\begin{array}{l}\text { Contract Receipts } \\
\text { (percent) }\end{array}$} & \multicolumn{2}{|c|}{$\begin{array}{l}\text { Sulfur Content } \\
\text { (lbs. sulfur } \\
\text { per MM Btu) }\end{array}$} & \multicolumn{2}{|c|}{$\begin{array}{c}\text { Price } \\
\text { (cents per MM Btu) }\end{array}$} \\
\hline & 1998 & 1997 & 1998 & 1997 & 1998 & 1997 & 1998 & 1997 \\
\hline \multicolumn{9}{|l|}{ Missouri } \\
\hline 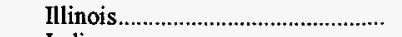 & 1,042 & 1,291 & 33.8 & 80.3 & 1.76 & 2.10 & 129 & 127 \\
\hline 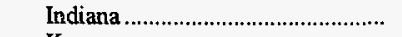 & 53 & - & - & - & .60 & - & 131 & - \\
\hline 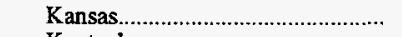 & - & 38 & - & 98.2 & - & 2.87 & - & 133 \\
\hline Kentucky & 23 & 23 & 35.4 & 100.0 & 2.05 & .70 & 182 & 214 \\
\hline 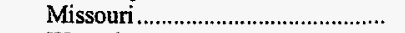 & 61 & 49 & 97.5 & 93.8 & 2.75 & 2.46 & 119 & 123 \\
\hline 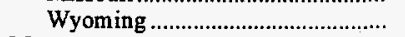 & 17,759 & 14,510 & 63.8 & 85.3 & .31 & .33 & 88 & 90 \\
\hline 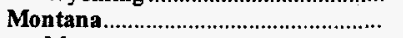 & 4,921 & 3,894 & 100.0 & 100.0 & .87 & .88 & 71 & 69 \\
\hline 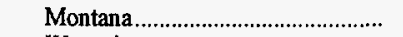 & 4,868 & 3,694 & 100.0 & 100.0 & .88 & .91 & 72 & 70 \\
\hline 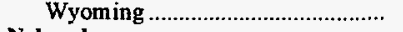 & 53 & 200 & 100.0 & 100.0 & .29 & .28 & 62 & 49 \\
\hline Nebraska & 5,858 & 5,349 & 80.4 & 75.2 & .32 & .36 & 59 & 60 \\
\hline 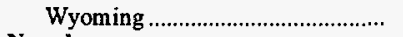 & 5,858 & 5,349 & 80.4 & 75.2 & .32 & .36 & 59 & 60 \\
\hline Nevada & 3,594 & 3,268 & 92.3 & 94.5 & .43 & .44 & 140 & 142 \\
\hline Arizona ............................................. & 2,128 & 2,133 & 100.0 & 100.0 & .48 & .46 & 123 & 135 \\
\hline 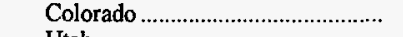 & 20 & 48 & 100.0 & 100.0 & .41 & .49 & 196 & 201 \\
\hline Utah & 1,446 & 1,070 & 80.9 & 84.8 & .37 & .41 & 161 & 155 \\
\hline Wyoming & - & 17 & - & - & - & .48 & - & 95 \\
\hline 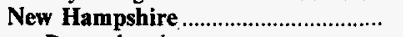 & 645 & 795 & 69.5 & 59.3 & 1.07 & 1.13 & 161 & 161 \\
\hline 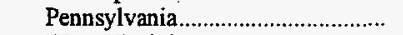 & 345 & 455 & 95.7 & 71.7 & 1.10 & 1.10 & 166 & 165 \\
\hline 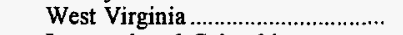 & 117 & 213 & 100.0 & 68.2 & 1.81 & 1.53 & 160 & 151 \\
\hline Imported coal Colombia ................ & 35 & - & - & - & .49 & - & 173 & - \\
\hline Imported coal Venezuela ............... & 148 & 127 & - & - & .51 & .57 & 147 & 162 \\
\hline 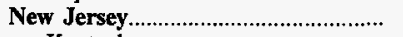 & 960 & 1,121 & 75.8 & 95.2 & .87 & .92 & 161 & 176 \\
\hline 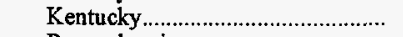 & 28 & 106 & - & 100.0 & .66 & .50 & 164 & 174 \\
\hline 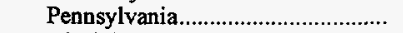 & * & - & 100.0 & - & .61 & - & 192 & - \\
\hline Virginia & 410 & 482 & 98.8 & 94.1 & .53 & .57 & 156 & 179 \\
\hline 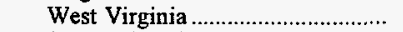 & 482 & 533 & 66.8 & 95.2 & 1.23 & 1.35 & 165 & 174 \\
\hline Imported coal Venezuela .............. & 39 & - & - & - & .52 & - & 155 & - \\
\hline 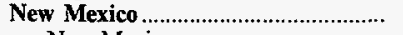 & 7,290 & 7,927 & 100.0 & 100.0 & .87 & .89 & 133 & 135 \\
\hline New Mexico & 7,290 & 7,927 & 100.0 & 100.0 & .87 & .89 & 133 & 135 \\
\hline New York & 4,300 & 3,643 & 87.0 & 87.4 & 1.25 & 1.32 & 144 & 141 \\
\hline 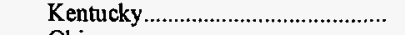 & 471 & 296 & 70.3 & 49.0 & .52 & .45 & 180 & 187 \\
\hline Ohio & 3 & - & - & - & 3.17 & - & 128 & - \\
\hline 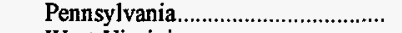 & 1,983 & 1,106 & 88.5 & 87.1 & 1.27 & 1.31 & 137 & 134 \\
\hline West Virginia & 1,581 & 2,083 & 88.2 & 92.1 & 1.56 & 1.52 & 137 & 136 \\
\hline Imported coal Colombia ................. & 35 & - & 100.0 & - & .47 & - & 170 & - \\
\hline Imported coal Venezuela ................ & 227 & 158 & 100.0 & 100.0 & .47 & .49 & 170 & 174 \\
\hline 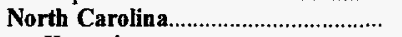 & 13,529 & 13,119 & 74.5 & 73.6 & .72 & .73 & 144 & 143 \\
\hline 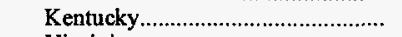 & 7,693 & 7,936 & 66.4 & 66.4 & .78 & .79 & 142 & 141 \\
\hline 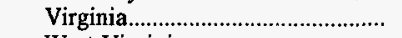 & 143 & 161 & .1 & - & .76 & .91 & 134 & 131 \\
\hline 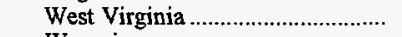 & 5,693 & 4,982 & 87.4 & 88.2 & .65 & .62 & 149 & 148 \\
\hline 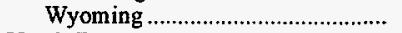 & - & 40 & - & - & - & .56 & - & 179 \\
\hline North Dakota & 11,423 & 11,199 & 100.0 & 98.1 & 1.16 & 1.15 & 77 & 76 \\
\hline 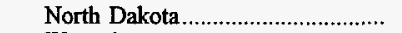 & 11,423 & 11,091 & 100.0 & 99.1 & 1.16 & 1.16 & 77 & 76 \\
\hline 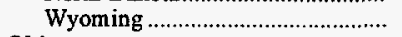 & - & 107 & - & - & - & .52 & - & 65 \\
\hline Ohio & 26,575 & 25,886 & 72.9 & 69.7 & 1.73 & 1.71 & 137 & 132 \\
\hline 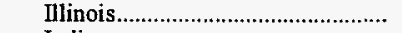 & 2 & - & - & - & .73 & - & 132 & - \\
\hline 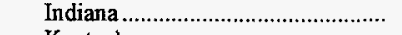 & 10 & 2 & - & - & 1.30 & 2.36 & 98 & 104 \\
\hline 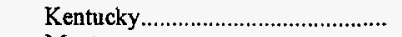 & 3,647 & 4,134 & 52.4 & 62.8 & .74 & .77 & 124 & 132 \\
\hline Montana & - & 15 & - & - & - & .32 & - & 149 \\
\hline Ohio & 10,247 & 10,840 & 83.6 & 74.8 & 2.97 & 2.87 & 146 & 133 \\
\hline 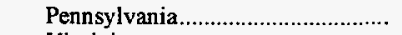 & 2,424 & 1,669 & 58.6 & 39.6 & 1.43 & 1.37 & 124 & 116 \\
\hline 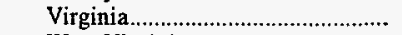 & 289 & 183 & 100.0 & 100.0 & .55 & .60 & 126 & 126 \\
\hline 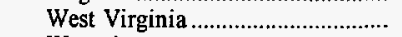 & 9,105 & 8,605 & 77.2 & 72.9 & .97 & .87 & 137 & 136 \\
\hline 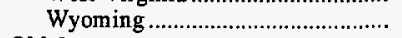 & 851 & 438 & 16.9 & 49.8 & .36 & .26 & 120 & 124 \\
\hline Oklahoma & 10,144 & 9,366 & 98.6 & 99.9 & .33 & .35 & 92 & 93 \\
\hline 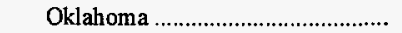 & 30 & 42 & 100.0 & 100.0 & 2.79 & 2.61 & 104 & 103 \\
\hline 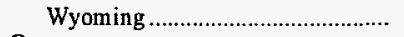 & 10,114 & 9,324 & 98.6 & 99.9 & .32 & .33 & 92 & 93 \\
\hline Oregon & 965 & 135 & - & - & .37 & .25 & 109 & 114 \\
\hline 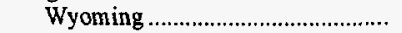 & 965 & 135 & - & - & .37 & .25 & 109 & 114 \\
\hline Pennsylvania & 21,836 & 21,881 & 83.2 & 74.9 & 1.79 & 1.71 & 136 & 137 \\
\hline 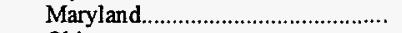 & 14 & 8 & - & - & .91 & .86 & 132 & 133 \\
\hline Ohio & 79 & 195 & 100.0 & 100.0 & 3.02 & 3.08 & 170 & 171 \\
\hline 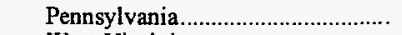 & 16,900 & 17,244 & 79.2 & 70.0 & 1.57 & 1.55 & 131 & 132 \\
\hline West Virginia & 4,844 & 4,434 & 97.3 & 93.1 & 2.52 & 2.27 & 154 & 154 \\
\hline 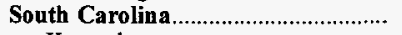 & 6,484 & 5,734 & 80.9 & 74.9 & .93 & .92 & 145 & 145 \\
\hline 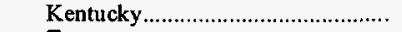 & 5,610 & 4,936 & 78.1 & 73.1 & .92 & .89 & 143 & 144 \\
\hline 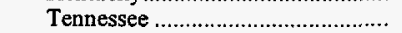 & 183 & 189 & 100.0 & 84.6 & 1.06 & 1.19 & 152 & 152 \\
\hline 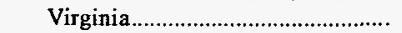 & 546 & 555 & 100.0 & 95.6 & 1.01 & 1.10 & 150 & 153 \\
\hline 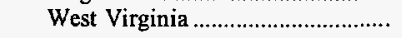 & 145 & 55 & 94.8 & - & .77 & 1.06 & 160 & 150 \\
\hline
\end{tabular}

See footnotes at end of table. 
Table 28. Destination of Coal Received at Electric Utility Plants by Origin, January-June 1998, 1997 (Continued)

\begin{tabular}{|c|c|c|c|c|c|c|c|c|}
\hline \multirow{2}{*}{$\begin{array}{l}\text { State of Destination } \\
\text { State of Origin } \\
\text { and Imports }\end{array}$} & \multicolumn{2}{|c|}{$\begin{array}{c}\text { Receipts } \\
\text { (thousand short tons) }\end{array}$} & \multicolumn{2}{|c|}{$\begin{array}{c}\text { Contract Receipts } \\
\text { (percent) }\end{array}$} & \multicolumn{2}{|c|}{$\begin{array}{l}\text { Sulfur Content } \\
\text { (lbs. sulfur } \\
\text { per MM Btu) }\end{array}$} & \multicolumn{2}{|c|}{$\begin{array}{c}\text { Price } \\
\text { (cents per MM Btu) }\end{array}$} \\
\hline & 1998 & 1997 & 1998 & 1997 & 1998 & 1997 & 1998 & 1997 \\
\hline 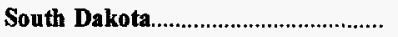 & 939 & 945 & 100.0 & 100.0 & $\mathbf{0 , 7 0}$ & 0.72 & 93 & 93 \\
\hline Montana & 939 & 945 & 100.0 & 100.0 & .70 & .72 & 93 & 93 \\
\hline 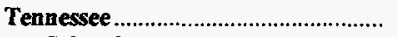 & 13,048 & 12,616 & 75.6 & 71.1 & 1.53 & 1.60 & 113 & 113 \\
\hline Colorado & 332 & 535 & 100.0 & 87.7 & .41 & .45 & 115 & 116 \\
\hline 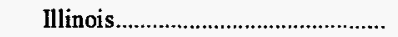 & 1,336 & 758 & 48.8 & 62.0 & 1.76 & 1.85 & 109 & 108 \\
\hline 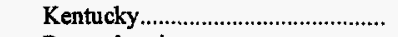 & 7,488 & 7,839 & 34.8 & 65.2 & 1.86 & 1.91 & 113 & 112 \\
\hline Pennsylvania & 270 & 317 & 94.6 & 100.0 & 1.76 & 1.88 & 110 & 110 \\
\hline 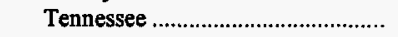 & 750 & 798 & 99.2 & 93.3 & 1.16 & .91 & 116 & 118 \\
\hline Utah & 722 & 654 & 46.1 & 96.5 & .48 & .47 & 124 & 122 \\
\hline 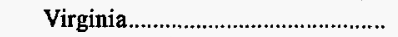 & 779 & 1,003 & 96.5 & 79.0 & 1.23 & 1.39 & 129 & 126 \\
\hline 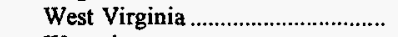 & 12 & - & - & - & .60 & - & 123 & - \\
\hline 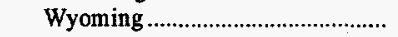 & 1,357 & 712 & 88.0 & 61.9 & .38 & .40 & 91 & 92 \\
\hline 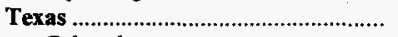 & 45,855 & 44,847 & 92,6 & 93.8 & .95 & .94 & 129 & 128 \\
\hline 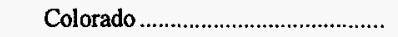 & 625 & 614 & 77.2 & - & .34 & .35 & 140 & 131 \\
\hline 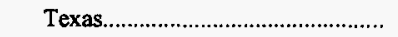 & 23,112 & 23,766 & 100.0 & 100.0 & 1.67 & 1.56 & 104 & 100 \\
\hline 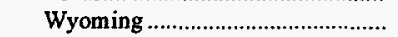 & 22,061 & 20,466 & 85.6 & 89.4 & .42 & .42 & 149 & 152 \\
\hline Imported coal Colombia .................. & 48 & - & - & - & .41 & - & 194 & - \\
\hline Imported coal Venezuela ................ & 9 & - & - & - & .54 & - & 186 & - \\
\hline Utah & 7,638 & 7,837 & 98.1 & 92.9 & .42 & .42 & 115 & 112 \\
\hline 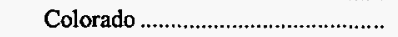 & 899 & 1,086 & 94.9 & 83.0 & .43 & .37 & 190 & 173 \\
\hline 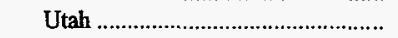 & 6,739 & 6,729 & 98.6 & 94.9 & .42 & .42 & 107 & 103 \\
\hline Wyoming & - & 22 & - & - & - & .27 & - & 128 \\
\hline Virginia & 6,010 & 5,700 & 69.4 & 76.1 & .78 & .81 & 138 & 139 \\
\hline 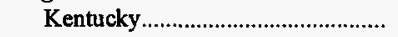 & 1,538 & 1,290 & 53.1 & 54.1 & .92 & .90 & 142 & 145 \\
\hline 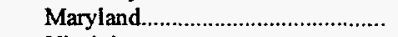 & - & 30 & - & - & - & 1.35 & - & 152 \\
\hline 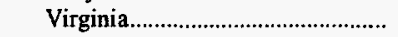 & 3,126 & 3,077 & 77.5 & 79.0 & .76 & .79 & 134 & 135 \\
\hline 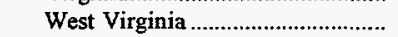 & 1,346 & 1,304 & 69.3 & 92.7 & .66 & .73 & 144 & 143 \\
\hline 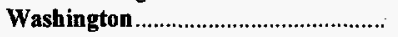 & 2,827 & 1,874 & $\mathbf{7 3 . 5}$ & 99.8 & .66 & .73 & 151 & 193 \\
\hline 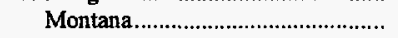 & 747 & 123 & - & 100.0 & .36 & .38 & 121 & 134 \\
\hline 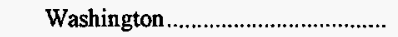 & 2,080 & 1,747 & 100.0 & 100.0 & .79 & .77 & 164 & 198 \\
\hline Imported coal Canada ....................... & - & 4 & - & - & - & .36 & - & 172 \\
\hline 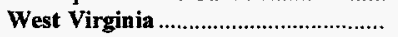 & 17,224 & 15,994 & 87.5 & 92.7 & 1.57 & 1.61 & 122 & 124 \\
\hline 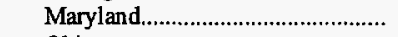 & 1,642 & 1,282 & 93.7 & 97.2 & 1.42 & 1.42 & 109 & 107 \\
\hline Ohio & 697 & 773 & 100.0 & 100.0 & 3.04 & 3.09 & 89 & 87 \\
\hline Pennsylvania & 1,384 & 1,060 & 92.8 & 86.5 & 1.23 & 1.17 & 127 & 125 \\
\hline 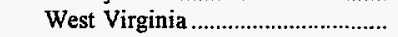 & 13,501 & 12,879 & 85.5 & 92.3 & 1.54 & 1.57 & 125 & 128 \\
\hline 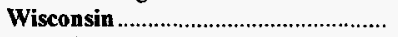 & 11,488 & 11,600 & 76.6 & 82.2 & .50 & .51 & 107 & 109 \\
\hline Colorado & - & 317 & - & - & - & .46 & - & 135 \\
\hline Illinois & 389 & 361 & - & - & .79 & .87 & 136 & 133 \\
\hline 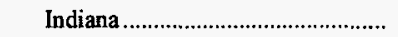 & 62 & 107 & - & - & 1.29 & 1.14 & 141 & 136 \\
\hline 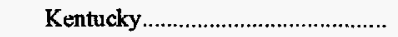 & 21 & 58 & - & - & .63 & .71 & 192 & 170 \\
\hline Montana & 816 & 1,091 & 100.0 & 99.2 & .65 & .61 & 110 & 111 \\
\hline 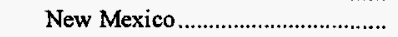 & 216 & 265 & 100.0 & 100.0 & .50 & .46 & 153 & 157 \\
\hline 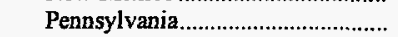 & 1,045 & 739 & 30.9 & 61.0 & 1.17 & 1.21 & 146 & 147 \\
\hline Utah & - & 52 & - & - & - & .45 & - & 165 \\
\hline 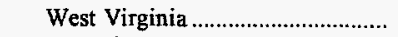 & - & 12 & - & - & - & .56 & - & 145 \\
\hline 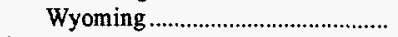 & 8,938 & 8,599 & 83.2 & 90.0 & .35 & .37 & 96 & 97 \\
\hline 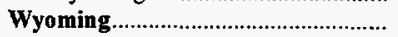 & 12,705 & 11,111 & 92.6 & 95.9 & .61 & .61 & 75 & 81 \\
\hline 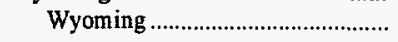 & 12,705 & 11,111 & 92.6 & 95.9 & .61 & 61 & 75 & 81 \\
\hline U.S. Total & 452,348 & 428,751 & 81.3 & 82.8 & 1.06 & 1.08 & 126 & 129 \\
\hline
\end{tabular}

* For quantity data, the number is less than 0.5 thousand short tons. For Contract Receipts (percent), the value is less than 0.05 .

Notes: Total may not equal sum of components because of independent rounding. MM Btu represents million Btu.

Source: Federal Energy Regulatory Commission, FERC Form 423, "Monthly Report of Cost and Quality of Fuels for Electric Plants." 
Table 29. Origin of Coal Received at Electric Utility Plants by Destination, January-June 1998, 1997

\begin{tabular}{|c|c|c|c|c|c|c|c|c|}
\hline \multirow{2}{*}{$\begin{array}{l}\text { State of Origin and Imports } \\
\text { State of Destination }\end{array}$} & \multicolumn{2}{|c|}{$\begin{array}{l}\text { Receipts } \\
\text { (thousand short tons) }\end{array}$} & \multicolumn{2}{|c|}{$\begin{array}{c}\text { Contract Receipts } \\
\text { (percent) }\end{array}$} & \multicolumn{2}{|c|}{$\begin{array}{c}\text { Sulfur Content } \\
\text { (lbs. sulfur } \\
\text { per MM Btu) }\end{array}$} & \multicolumn{2}{|c|}{$\begin{array}{c}\text { Price } \\
\text { (cents per MM Btu) }\end{array}$} \\
\hline & 1998 & 1997 & 1998 & 1997 & 1998 & 1997 & 1998 & 1997 \\
\hline Alabama....... & 7,636 & 8,517 & 91.7 & 89.2 & 0.95 & 0.92 & 183 & 178 \\
\hline 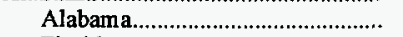 & 7,499 & 8,228 & 93.4 & 92.4 & .95 & .89 & 183 & 179 \\
\hline Florida. & 109 & 193 & - & - & .88 & 1.94 & 162 & 176 \\
\hline Georgia & - & 50 & - & - & - & 1.51 & - & 135 \\
\hline 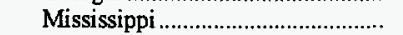 & 28 & 46 & - & - & .57 & .63 & 148 & 138 \\
\hline 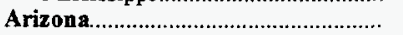 & 5,839 & 4,952 & 100.0 & 100.0 & .49 & .48 & 120 & 129 \\
\hline Arizona & 3,711 & 2,819 & 100.0 & 100.0 & .49 & .49 & 118 & 125 \\
\hline Nevada & 2,128 & 2,133 & 100.0 & 100.0 & .48 & .46 & 123 & 135 \\
\hline 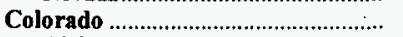 & 10,897 & 11,490 & 93.0 & 82.0 & .41 & .41 & 123 & 124 \\
\hline 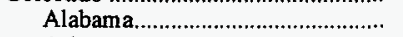 & 340 & 653 & 100.0 & 100.0 & .42 & .44 & 116 & 113 \\
\hline Arizona & 30 & - & - & - & .40 & - & 154 & - \\
\hline Colorado & 5,488 & 5,299 & 93.1 & 90.1 & .41 & .41 & 106 & 111 \\
\hline 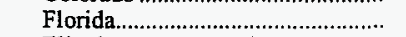 & - & 14 & - & - & - & .54 & - & 183 \\
\hline 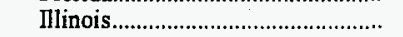 & 690 & 611 & 83.2 & 55.0 & .45 & .47 & 138 & 136 \\
\hline Iowa & 249 & 260 & 100.0 & 88.4 & .43 & .46 & 132 & 135 \\
\hline 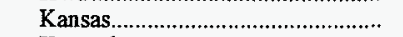 & 603 & 842 & 100.0 & 100.0 & .42 & .37 & 122 & 125 \\
\hline 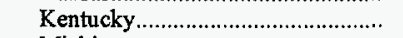 & 1,302 & 993 & 100.0 & 100.0 & .40 & .43 & 126 & 129 \\
\hline 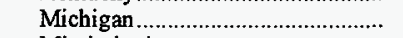 & 288 & 182 & 94.0 & 94.4 & .48 & .46 & 138 & 135 \\
\hline 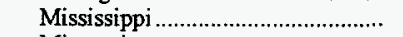 & - & 36 & - & - & - & .42 & - & 155 \\
\hline 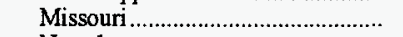 & 30 & - & - & - & .54 & - & 137 & - \\
\hline 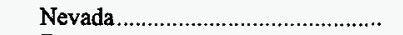 & 20 & 48 & 100.0 & 100.0 & .41 & .49 & 196 & 201 \\
\hline Tennessee & 332 & 535 & 100.0 & 87.7 & .41 & .45 & 115 & 116 \\
\hline 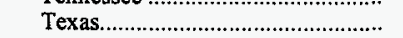 & 625 & 614 & 77.2 & - & .34 & .35 & 140 & 131 \\
\hline Utah & 899 & 1,086 & 94.9 & 83.0 & .43 & .37 & 190 & 173 \\
\hline 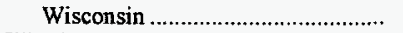 & - & 317 & - & - & - & .46 & - & 135 \\
\hline Illinois & 17,400 & 17,815 & $\mathbf{7 2 . 7}$ & 76.3 & 2.00 & 2.04 & 134 & 137 \\
\hline 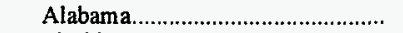 & 435 & 854 & 14.0 & - & 1.72 & 1.20 & 128 & 130 \\
\hline 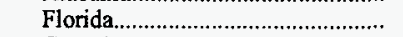 & 3,529 & 3,035 & 80.9 & 77.7 & 1.86 & 1.87 & 167 & 184 \\
\hline 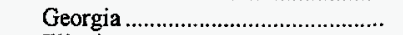 & 400 & 450 & 100.0 & 89.6 & 1.27 & .87 & 137 & 147 \\
\hline 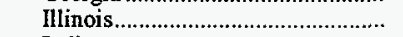 & 6,920 & 7,374 & 88.1 & 82.8 & 2.33 & 2.49 & 127 & 124 \\
\hline Indiana & 2,338 & 2,789 & 65.0 & 89.6 & 2.17 & 1.99 & 119 & 131 \\
\hline Iowa & 102 & 47 & 28.0 & 72.1 & 2.32 & 2.13 & 117 & 128 \\
\hline 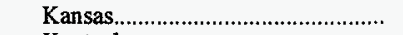 & - & 88 & - & 100.0 & - & 2.69 & - & 107 \\
\hline 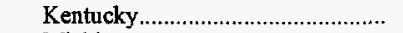 & 143 & 91 & - & - & 2.04 & 2.35 & 100 & 104 \\
\hline 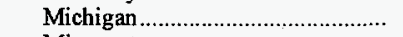 & 9 & - & - & - & 1.08 & - & 152 & - \\
\hline 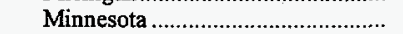 & 11 & 52 & 100.0 & 100.0 & 1.10 & 1.12 & 161 & 166 \\
\hline 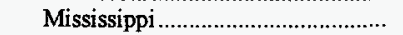 & 744 & 626 & 89.6 & 87.9 & 1.39 & 1.02 & 142 & 138 \\
\hline 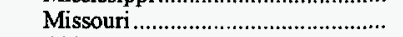 & 1,042 & 1,291 & 33.8 & 80.3 & 1.76 & 2.10 & 129 & 127 \\
\hline Ohio & 2 & - & - & - & .73 & - & 132 & - \\
\hline 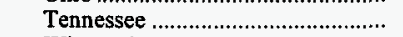 & 1,336 & 758 & 48.8 & 62.0 & 1.76 & 1.85 & 109 & 108 \\
\hline Wisconsin & 389 & 361 & - & - & .79 & .87 & 136 & 133 \\
\hline Indiana & 16,205 & 13,941 & 68.2 & 59.9 & 2.07 & 2.13 & 110 & 107 \\
\hline Alabama & 49 & - & - & - & 3.12 & - & 110 & - \\
\hline Florida & 30 & 5 & 100.0 & - & 2.79 & .62 & 125 & 164 \\
\hline Illinois & 836 & 825 & 70.6 & 49.9 & .84 & .95 & 147 & 140 \\
\hline Indiana & 13,879 & 11,646 & 66.5 & 57.1 & 2.10 & 2.15 & 109 & 106 \\
\hline Iowa & 90 & 66 & 100.0 & 24.5 & 1.23 & 1.28 & 125 & 123 \\
\hline Kentucky & 1,074 & 1,222 & 93.9 & 99.3 & 2.88 & 2.82 & 95 & 91 \\
\hline Michigan & 106 & 69 & 100.0 & 100.0 & 2.11 & 2.01 & 138 & 136 \\
\hline Minnesota & 15 & - & - & - & 1.13 & - & 150 & - \\
\hline 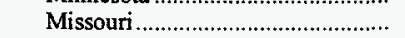 & 53 & - & - & - & .60 & - & 131 & - \\
\hline Ohio & 10 & 2 & - & - & 1.30 & 2.36 & 98 & 104 \\
\hline 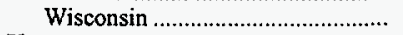 & 62 & 107 & - & - & 1.29 & 1.14 & 141 & 136 \\
\hline 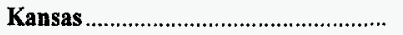 & 202 & 122 & 100.0 & 99.4 & 3.86 & 3.08 & 103 & 120 \\
\hline 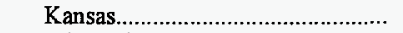 & 202 & 84 & 100.0 & 100.0 & 3.86 & 3.17 & 103 & 115 \\
\hline 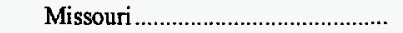 & - & 38 & - & 98.2 & - & 2.87 & - & 133 \\
\hline 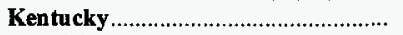 & 60,278 & 59,259 & 63.7 & 66.7 & 1.33 & 1.38 & 134 & 136 \\
\hline 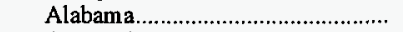 & 2,121 & 1,344 & 65.1 & 66.5 & 1.65 & 1.95 & 120 & 112 \\
\hline 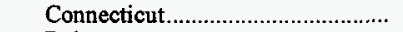 & 352 & 405 & 87.8 & 98.8 & .38 & .41 & 183 & 193 \\
\hline 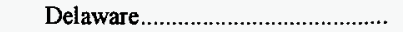 & 54 & - & 100.0 & - & .50 & - & 176 & - \\
\hline Florida & 7,078 & 7,113 & 64.3 & 62.2 & 1.21 & 1.30 & 168 & 175 \\
\hline Georgia & 7,249 & 7,294 & 54.9 & 65.6 & .82 & .77 & 150 & 155 \\
\hline Illinois & 9 & 88 & - & 20.5 & .57 & .45 & 169 & 172 \\
\hline Indiana & 668 & 454 & 56.4 & 84.1 & 1.37 & 1.36 & 122 & 125 \\
\hline Iowa & 55 & 31 & 59.8 & - & 2.35 & 2.30 & 117 & 112 \\
\hline 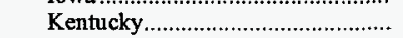 & 12,969 & 12,704 & 55.0 & 68.8 & 2.37 & 2.53 & 103 & 101 \\
\hline 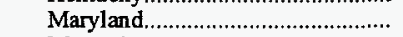 & 259 & 269 & 100.0 & 85.9 & .58 & .58 & 146 & 151 \\
\hline 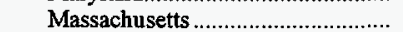 & 388 & 405 & 97.9 & 91.7 & .53 & .55 & 181 & 181 \\
\hline 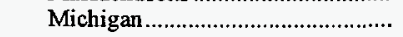 & 2,173 & 1,993 & 64.2 & 63.8 & .68 & .72 & 150 & 154 \\
\hline
\end{tabular}

See footnotes at end of table. 
Table 29. Origin of Coal Received at Electric Utility Plants by Destination, January-June 1998, 1997 (Continued)

\begin{tabular}{|c|c|c|c|c|c|c|c|c|}
\hline \multirow[t]{2}{*}{$\begin{array}{l}\text { State of Origin and Imports } \\
\text { State of Destination }\end{array}$} & \multicolumn{2}{|c|}{$\begin{array}{l}\text { Receipts } \\
\text { (thousand short tons) }\end{array}$} & \multicolumn{2}{|c|}{$\begin{array}{c}\text { Contract Receipts } \\
\text { (percent) }\end{array}$} & \multicolumn{2}{|c|}{$\begin{array}{c}\text { Sulfur Content } \\
\text { (Ibs. sulfur } \\
\text { per MM Btu) }\end{array}$} & \multicolumn{2}{|c|}{$\begin{array}{c}\text { Price } \\
\text { (cents per MM Btu) }\end{array}$} \\
\hline & 1998 & 1997 & 1998 & 1997 & 1998 & 1997 & 1998 & 1997 \\
\hline \multicolumn{9}{|l|}{ Kentucky } \\
\hline Mississippi & 384 & 541 & 96.0 & 87.7 & 0.76 & 0.80 & 203 & 198 \\
\hline 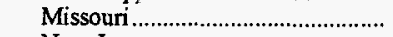 & 23 & 23 & 35.4 & 100.0 & 2.05 & .70 & 182 & 214 \\
\hline 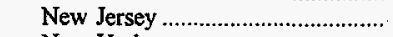 & 28 & 106 & - & 100.0 & .66 & .50 & 164 & 174 \\
\hline 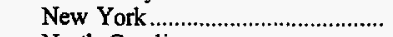 & 471 & 296 & 70.3 & 49.0 & .52 & .45 & 180 & 187 \\
\hline 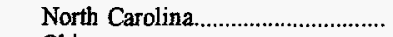 & 7,693 & 7,936 & 66.4 & 66.4 & .78 & .79 & 142 & 141 \\
\hline Ohio & 3,647 & 4,134 & 52.4 & 62.8 & .74 & .77 & 124 & 132 \\
\hline 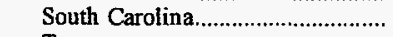 & 5,610 & 4,936 & 78.1 & 73.1 & .92 & .89 & 143 & 144 \\
\hline 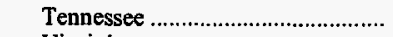 & 7,488 & 7,839 & 74.8 & 65.2 & 1.86 & 1.91 & 113 & 112 \\
\hline 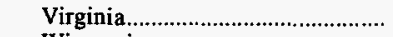 & 1,538 & 1,290 & 53.1 & 54.1 & .92 & .90 & 142 & 145 \\
\hline Wisconsin & 21 & 58 & - & - & .63 & .71 & 192 & 170 \\
\hline Louisiana & 1,551 & 1,817 & 100.0 & 100.0 & 1.47 & 1.54 & 137 & 134 \\
\hline 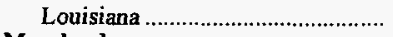 & 1,551 & 1,817 & 100.0 & 100.0 & 1.47 & 1.54 & 137 & 134 \\
\hline 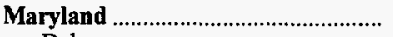 & 2,056 & 1,752 & 91.6 & 92.5 & 1.37 & 1.33 & 122 & 124 \\
\hline 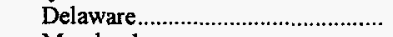 & 57 & 39 & 100.0 & 100.0 & 1.13 & 1.09 & 146 & 149 \\
\hline Maryland & 343 & 393 & 84.2 & 85.0 & 1.15 & 1.09 & 175 & 173 \\
\hline Pennsylvania & 14 & 8 & - & - & .91 & .86 & 132 & 133 \\
\hline 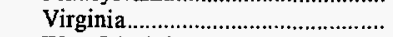 & - & 30 & - & - & - & 1.35 & - & 152 \\
\hline 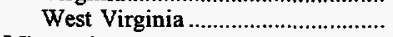 & 1,642 & 1,282 & 93.7 & 97.2 & 1.42 & 1.42 & 109 & 107 \\
\hline 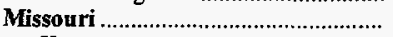 & 75 & 201 & 98.0 & 98.5 & 2.81 & 3.47 & 121 & 106 \\
\hline 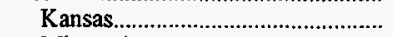 & 14 & 152 & 100.0 & 100.0 & 3.06 & 3.79 & 126 & 101 \\
\hline 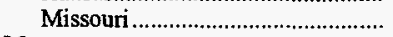 & 61 & 49 & 97.5 & 93.8 & 2.75 & 2.46 & 119 & 123 \\
\hline 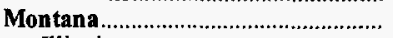 & 18,142 & 16,165 & 92.4 & 98.0 & .61 & .60 & 116 & 127 \\
\hline Illinois & 655 & 790 & 100.0 & 100.0 & .34 & .33 & 234 & 262 \\
\hline 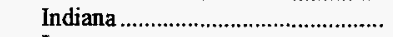 & - & 604 & - & 100.0 & - & .33 & - & 253 \\
\hline Iowa & 50 & 29 & 44.6 & - & .30 & .30 & 135 & 148 \\
\hline 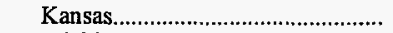 & 67 & - & 100.0 & - & .34 & - & 91 & - \\
\hline 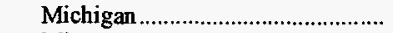 & 3,866 & 3,025 & 89.2 & 100.0 & .39 & .39 & 143 & 157 \\
\hline Minnesota & 4,526 & 4,320 & 96.1 & 93.8 & .68 & .66 & 111 & 112 \\
\hline Mississippi & 1,606 & 1,528 & 100.0 & 100.0 & .40 & .43 & 147 & 143 \\
\hline Montana & 4,868 & 3,694 & 100.0 & 100.0 & .88 & .91 & 72 & 70 \\
\hline Ohio & - & 15 & - & - & - & .32 & - & 149 \\
\hline 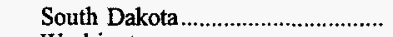 & 939 & 945 & 100.0 & 100.0 & .70 & .72 & 93 & 93 \\
\hline 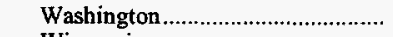 & 747 & 123 & - & 100.0 & .36 & .38 & 121 & 134 \\
\hline 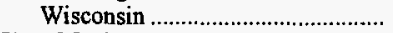 & 816 & 1,091 & 100.0 & 99.2 & .65 & .61 & 110 & 111 \\
\hline 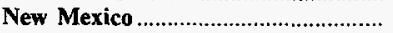 & 12,673 & 13,052 & 91.7 & 90.9 & .74 & .75 & 140 & 146 \\
\hline 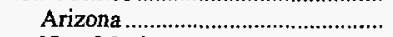 & 5,167 & 4,860 & 79.6 & 75.5 & .58 & .55 & 147 & 161 \\
\hline 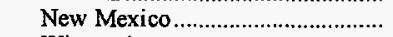 & 7,290 & 7,927 & 100.0 & 100.0 & .87 & .89 & 133 & 135 \\
\hline 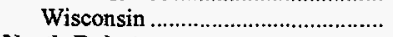 & 216 & 265 & 100.0 & 100.0 & .50 & .46 & 153 & 157 \\
\hline 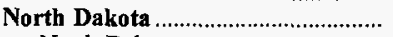 & 11,423 & 11,091 & 100.0 & 99.1 & 1.16 & 1.16 & 77 & 76 \\
\hline 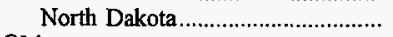 & 11,423 & 11,091 & 100.0 & 99.1 & 1.16 & 1.16 & 77 & 76 \\
\hline 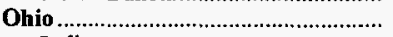 & 12,019 & 12,789 & $\mathbf{8 0 . 5}$ & 72.4 & 3.00 & 2.94 & 140 & 128 \\
\hline Indiana & 562 & 613 & - & - & 3.38 & 3.57 & 109 & 101 \\
\hline 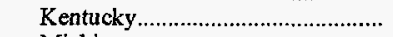 & 357 & 316 & 87.9 & 41.7 & 3.40 & 3.68 & 96 & 89 \\
\hline 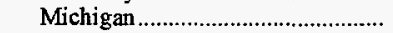 & 74 & 52 & 18.8 & 97.6 & 2.66 & 2.50 & 157 & 155 \\
\hline 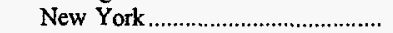 & 3 & - & - & - & 3.17 & - & 128 & - \\
\hline Ohio & 10,247 & 10,840 & 83.6 & 74.8 & 2.97 & 2.87 & 146 & 133 \\
\hline 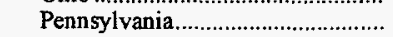 & 79 & 195 & 100.0 & 100.0 & 3.02 & 3.08 & 170 & 171 \\
\hline 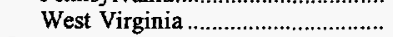 & 697 & 773 & 100.0 & 100.0 & 3.04 & 3.09 & 89 & 87 \\
\hline Oklahoma & 68 & 50 & 100.0 & 83.9 & 2.85 & 2.66 & 112 & 106 \\
\hline 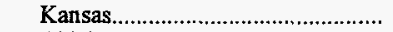 & 38 & 8 & 100.0 & - & 2.90 & 2.91 & 118 & 120 \\
\hline Oklahoma & 30 & 42 & 100.0 & 100.0 & 2.79 & 2.61 & 104 & 103 \\
\hline 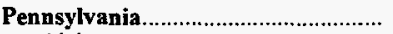 & 28,293 & 25,066 & 73.5 & 70.3 & 1.45 & 1.46 & 132 & 131 \\
\hline 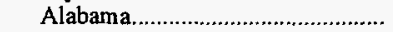 & 29 & 46 & 100.0 & 100.0 & 1.53 & 1.80 & 112 & 112 \\
\hline 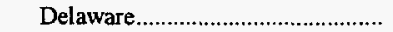 & 240 & 217 & 97.7 & 59.4 & 1.04 & 1.02 & 143 & 142 \\
\hline Indiana & 283 & 264 & 100.0 & 98.7 & 1.67 & 1.75 & 107 & 104 \\
\hline 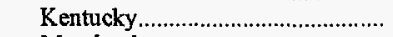 & 48 & 57 & 80.7 & 64.0 & 1.72 & 1.65 & 110 & 111 \\
\hline 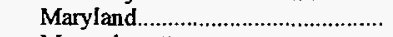 & 1,516 & 554 & 16.4 & 61.2 & 1.13 & 1.06 & 149 & 157 \\
\hline 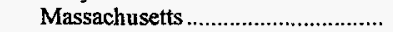 & 87 & . 125 & 100.0 & 73.0 & 1.19 & .99 & 161 & 166 \\
\hline 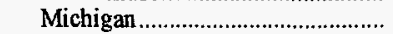 & 1,737 & 1,213 & 64.8 & 84.3 & 1.16 & 1.18 & 125 & 121 \\
\hline New Hampshire & 345 & 455 & 95.7 & 71.7 & 1.10 & 1.10 & 166 & 165 \\
\hline 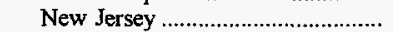 & $*$ & - & 100.0 & - & .61 & - & 192 & - \\
\hline New York & 1,983 & 1,106 & 88.5 & 87.1 & 1.27 & 1.31 & 137 & 134 \\
\hline Ohio & 2,424 & 1,669 & 58.6 & 39.6 & 1.43 & 1.37 & 124 & 116 \\
\hline 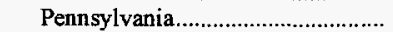 & 16,900 & 17,244 & 79.2 & 70.0 & 1.57 & 1.55 & 131 & 132 \\
\hline Tennessee & 270 & 317 & 94.6 & 100.0 & 1.76 & 1.88 & 110 & 110 \\
\hline 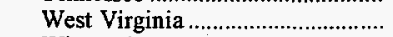 & 1,384 & 1,060 & 92.8 & 86.5 & 1.23 & 1.17 & 127 & 125 \\
\hline 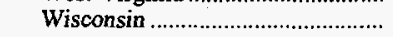 & 1,045 & 739 & 30.9 & 61.0 & 1.17 & 1.21 & 146 & 147 \\
\hline
\end{tabular}

See footnotes at end of table. 
Table 29. Origin of Coal Received at Electric Utility Plants by Destination, January-June 1998, 1997 (Continued)

\begin{tabular}{|c|c|c|c|c|c|c|c|c|}
\hline \multirow{2}{*}{$\begin{array}{c}\text { State of Origin and Imports } \\
\text { State of Destination }\end{array}$} & \multicolumn{2}{|c|}{$\begin{array}{l}\text { Receipts } \\
\text { (thousand short tons) }\end{array}$} & \multicolumn{2}{|c|}{$\begin{array}{c}\text { Contract Receipts } \\
\text { (percent) }\end{array}$} & \multicolumn{2}{|c|}{$\begin{array}{c}\text { Sulfur Content } \\
\text { (lbs. sulfur } \\
\text { per MM Btu) }\end{array}$} & \multicolumn{2}{|c|}{$\begin{array}{c}\text { Price } \\
\text { (cents per MM Btu) }\end{array}$} \\
\hline & 1998 & 1997 & 1998 & 1997 & 1998 & 1997 & 1998 & 1997 \\
\hline 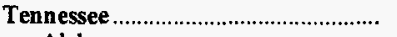 & 1,255 & 1,270 & 99.5 & 93.5 & 1.03 & 0.91 & 127 & 127 \\
\hline 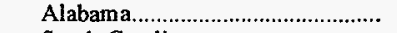 & 321 & 284 & 100.0 & 100.0 & .70 & .71 & 136 & 134 \\
\hline 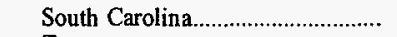 & 183 & 189 & 100.0 & 84.6 & 1.06 & 1.19 & 152 & 152 \\
\hline Tennessee & 750 & 798 & 99.2 & 93.3 & 1.16 & .91 & 116 & 118 \\
\hline Texas & 23,203 & 23,766 & 100.0 & 100.0 & 1.67 & 1.56 & 104 & 100 \\
\hline 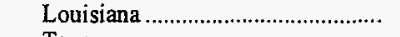 & 91 & - & 100.0 & - & 2.31 & - & 132 & - \\
\hline 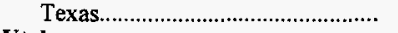 & 23,112 & 23,766 & 100.0 & 100.0 & 1.67 & 1.56 & 104 & 100 \\
\hline Utah & 9,342 & 9,639 & 91.4 & 89.9 & .41 & .42 & 120 & 117 \\
\hline 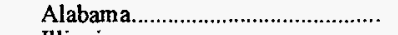 & - & 122 & $\vec{a}$ & 81.1 & - & .39 & - & 122 \\
\hline 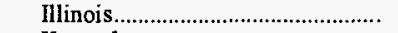 & 434 & 880 & 90.3 & 59.8 & .37 & .38 & 179 & 171 \\
\hline 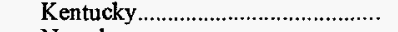 & - & 132 & - & 92.4 & - & .54 & - & 134 \\
\hline 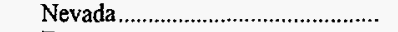 & 1,446 & 1,070 & 80.9 & 84.8 & .37 & .41 & 161 & 155 \\
\hline 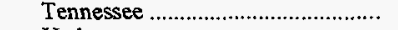 & 722 & 654 & 46.1 & 96.5 & .48 & .47 & 124 & 122 \\
\hline Utah & 6,739 & 6,729 & 98.6 & 94.9 & .42 & .42 & 107 & 103 \\
\hline 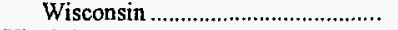 & - & 52 & - & - & - & .45 & - & 165 \\
\hline Virginia & 8,063 & 7,382 & 79.8 & 82.0 & .76 & .85 & 145 & 147 \\
\hline Connecticut..................................... & - & 4 & - & 100.0 & - & .52 & - & 184 \\
\hline Delaware & 65 & 76 & 100.0 & 88.3 & .61 & .67 & 175 & 158 \\
\hline Florida & 407 & 454 & 97.8 & 96.0 & .63 & .67 & 209 & 210 \\
\hline Georgia & 1,856 & 963 & 60.0 & 75.7 & .68 & .73 & 153 & 161 \\
\hline 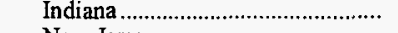 & 443 & 426 & 100.0 & 100.0 & .54 & .54 & 159 & 157 \\
\hline 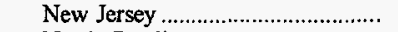 & 410 & 482 & 98.8 & 94.1 & .53 & .57 & 156 & 179 \\
\hline 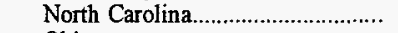 & 143 & 161 & .1 & - & .76 & .91 & 134 & 131 \\
\hline Ohio & 289 & 183 & 100.0 & 100.0 & .55 & .60 & 126 & 126 \\
\hline South Carolina................................... & 546 & 555 & 100.0 & 95.6 & 1.01 & 1.10 & 150 & 153 \\
\hline 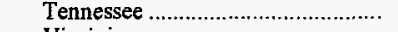 & 779 & 1,003 & 96.5 & 79.0 & 1.23 & 1.39 & 129 & 126 \\
\hline Virginia ........................................ & 3,126 & 3,077 & 77.5 & 79.0 & .76 & .79 & 134 & 135 \\
\hline 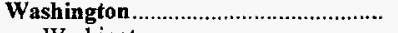 & 2,080 & 1,747 & 100.0 & 100.0 & .79 & .77 & 164 & 198 \\
\hline 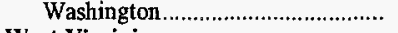 & 2,080 & 1,747 & 100.0 & 100.0 & .79 & .77 & 164 & 198 \\
\hline West Virginia .......................................... & 52,823 & 52,563 & 80.5 & 81.5 & 1.24 & 1.20 & 140 & 141 \\
\hline 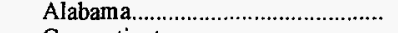 & 1,061 & 1,797 & 51.7 & 63.4 & 1.57 & 1.36 & 132 & 129 \\
\hline 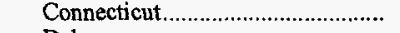 & 64 & 139 & 76.6 & 79.9 & .46 & .48 & 181 & 190 \\
\hline 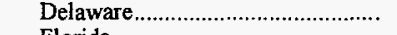 & 315 & 537 & 96.8 & 82.8 & .55 & .55 & 163 & 170 \\
\hline 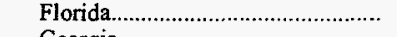 & 1,018 & 1,351 & 44.0 & 49.2 & 1.36 & 1.17 & 164 & 168 \\
\hline 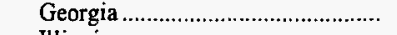 & 2,358 & 2,162 & 76.5 & 77.8 & .60 & .57 & 182 & 184 \\
\hline Illinois & - & 7 & - & - & - & .57 & - & 146 \\
\hline 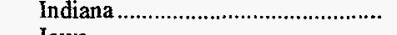 & 900 & 538 & 53.7 & 57.8 & .86 & .95 & 119 & 142 \\
\hline Iowa & 1 & 1 & - & - & 2.08 & 2.08 & 164 & 153 \\
\hline 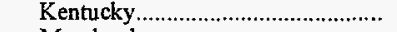 & 3,118 & 3,582 & 72.8 & 66.5 & 1.41 & 1.32 & 111 & 112 \\
\hline 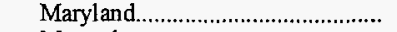 & 3,372 & 3,680 & 91.2 & 77.3 & .80 & .82 & 142 & 150 \\
\hline 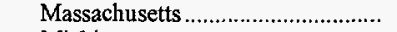 & 1,313 & 1,091 & 77.1 & 85.6 & .56 & .56 & 168 & 173 \\
\hline Michigan & 2,475 & 2,579 & 54.5 & 71.9 & .93 & .88 & 152 & 155 \\
\hline 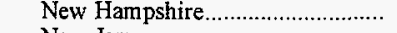 & 117 & 213 & 100.0 & 68.2 & 1.81 & 1.53 & 160 & 151 \\
\hline 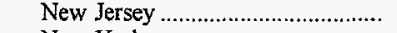 & 482 & 533 & 66.8 & 95.2 & 1.23 & 1.35 & 165 & 174 \\
\hline 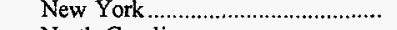 & 1,581 & 2,083 & 88.2 & 92.1 & 1.56 & 1.52 & 137 & 136 \\
\hline North Carolina................................... & 5,693 & 4,982 & 87.4 & 88.2 & .65 & .62 & 149 & 148 \\
\hline Ohio & 9,105 & 8,605 & 77.2 & 72.9 & .97 & .87 & 137 & 136 \\
\hline Pennsylvania & 4,844 & 4,434 & 97.3 & 93.1 & 2.52 & 2.27 & 154 & 154 \\
\hline South Carolina.................................... & 145 & 55 & 94.8 & - & .77 & 1.06 & 160 & 150 \\
\hline 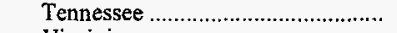 & 12 & - & - & - & .60 & - & 123 & - \\
\hline Virginia & 1,346 & 1,304 & 69.3 & 92.7 & .66 & .73 & 144 & 143 \\
\hline West Virginia ...................................... & 13,501 & 12,879 & 85.5 & 92.3 & 1.54 & 1.57 & 125 & 128 \\
\hline 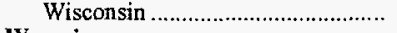 & - & 12 & - & - & - & .56 & - & 145 \\
\hline 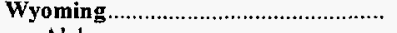 & 148,150 & 132,441 & 82.8 & 87.9 & .39 & .40 & 112 & 118 \\
\hline Alabama & 2,823 & 2,206 & 99.5 & 98.9 & .31 & .29 & 119 & 112 \\
\hline Arizona & 195 & - & - & - & .29 & - & 117 & - \\
\hline Arkansas & 6,580 & 5,865 & 89.6 & 93.2 & .38 & .38 & 149 & 169 \\
\hline Colorado & 3,355 & 2,841 & 93.4 & 91.6 & .34 & .32 & 88 & 89 \\
\hline 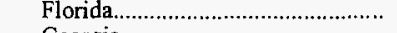 & 478 & 520 & - & - & .46 & .51 & 136 & 143 \\
\hline Georgia & 2,790 & 2,640 & - & - & .41 & .45 & 150 & 150 \\
\hline 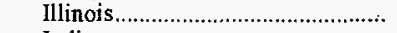 & 9,923 & 10,469 & 88.3 & 90.3 & .33 & .38 & 186 & 194 \\
\hline Indiana & 9,054 & 8,483 & 85.1 & 87.1 & .34 & .34 & 113 & 113 \\
\hline Iowa & 9,523 & 7,944 & 76.3 & 82.5 & .44 & .44 & 87 & 90 \\
\hline 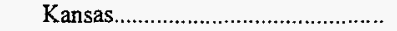 & 8,474 & 6,789 & 99.1 & 99.2 & .40 & .43 & 96 & 103 \\
\hline 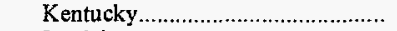 & 94 & 212 & 23.9 & 79.6 & .32 & .56 & 94 & 119 \\
\hline 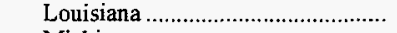 & 4,809 & 4,689 & 100.0 & 100.0 & .52 & .54 & 144 & 155 \\
\hline 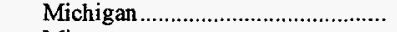 & 5,145 & 4,733 & 86.5 & 84.6 & .35 & .33 & 99 & 107 \\
\hline 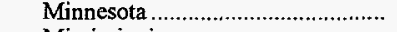 & 4,014 & 3,902 & 99.0 & 100.0 & .28 & .29 & 110 & 112 \\
\hline 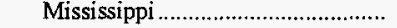 & 231 & 116 & - & - & .48 & .53 & 133 & 136 \\
\hline
\end{tabular}

See footnotes at end of table. 
Table 29. Origin of Coal Received at Electric Utility Plants by Destination, January-June 1998, 1997 (Continued)

\begin{tabular}{|c|c|c|c|c|c|c|c|c|}
\hline \multirow{2}{*}{$\begin{array}{l}\text { State of Origin and Imports } \\
\text { State of Destination }\end{array}$} & \multicolumn{2}{|c|}{$\begin{array}{l}\text { Receipts } \\
\text { (thousand short tons) }\end{array}$} & \multicolumn{2}{|c|}{$\begin{array}{c}\text { Contract Receipts } \\
\text { (percent) }\end{array}$} & \multicolumn{2}{|c|}{$\begin{array}{c}\text { Sulfur Content } \\
\text { (lbs. sulfur } \\
\text { per MM Btu) }\end{array}$} & \multicolumn{2}{|c|}{$\begin{array}{c}\text { Price } \\
\text { (cents per MM Btu) }\end{array}$} \\
\hline & 1998 & 1997 & 1998 & 1997 & 1998 & 1997 & 1998 & 1997 \\
\hline \multicolumn{9}{|l|}{ Wyoming } \\
\hline 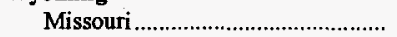 & 17,759 & 14,510 & 63.8 & 85.3 & 0.31 & 0.33 & 88 & 90 \\
\hline 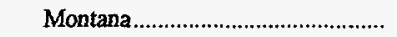 & 53 & 200 & 100.0 & 100.0 & .29 & .28 & 62 & 49 \\
\hline 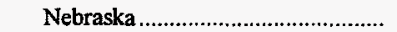 & 5,858 & 5,349 & 80.4 & 75.2 & .32 & .36 & 59 & 60 \\
\hline 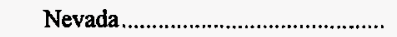 & - & 17 & - & - & - & .48 & - & 95 \\
\hline 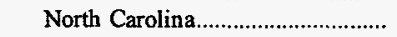 & - & 40 & - & - & - & .56 & - & 179 \\
\hline 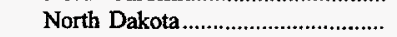 & - & 107 & - & - & - & .52 & - & 65 \\
\hline Ohio & 851 & 438 & 16.9 & 49.8 & .36 & .26 & 120 & 124 \\
\hline Oklahoma & 10,114 & 9,324 & 98.6 & 99.9 & .32 & .33 & 92 & 93 \\
\hline 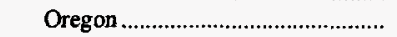 & 965 & 135 & - & - & .37 & .25 & 109 & 114 \\
\hline 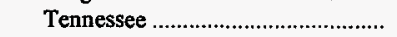 & 1,357 & 712 & 88.0 & 61.9 & .38 & .40 & 91 & 92 \\
\hline 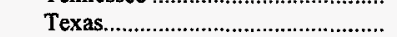 & 22,061 & 20,466 & 85.6 & 89.4 & .42 & .42 & 149 & 152 \\
\hline Utah & - & 22 & - & - & - & .27 & - & 128 \\
\hline 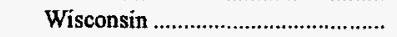 & 8,938 & 8,599 & 83.2 & 90.0 & .35 & .37 & 96 & 97 \\
\hline 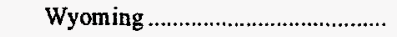 & 12,705 & 11,111 & 92.6 & 95.9 & .61 & .61 & 75 & 81 \\
\hline Imported Coal..................................... & 2,676 & 1,863 & 68.7 & 87.6 & .52 & .58 & 157 & 159 \\
\hline Canada & - & 4 & - & - & - & .36 & - & 172 \\
\hline Washington & - & 4 & - & - & - & .36 & - & 172 \\
\hline 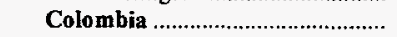 & 1,204 & 1,076 & 89.5 & 100.0 & .51 & .57 & 157 & 157 \\
\hline 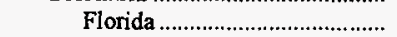 & 700 & 565 & 93.8 & 100.0 & .53 & .60 & 148 & 152 \\
\hline 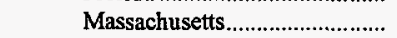 & 387 & 511 & 100.0 & 100.0 & .51 & .53 & 166 & 163 \\
\hline New Hampshire ......................... & 35 & - & - & - & .49 & - & 173 & - \\
\hline 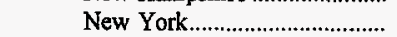 & 35 & - & 100.0 & - & .47 & - & 170 & - \\
\hline 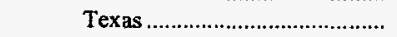 & 48 & - & - & - & .41 & - & 194 & - \\
\hline 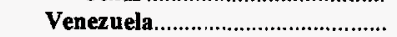 & 1,174 & 496 & 45.5 & 54.3 & .57 & .68 & 156 & 162 \\
\hline 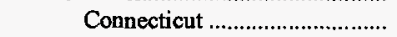 & 36 & - & - & - & .42 & - & 162 & - \\
\hline Florida & 193 & - & - & - & .60 & - & 156 & - \\
\hline 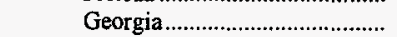 & 150 & 99 & - & - & .88 & 1.34 & 150 & 138 \\
\hline Massachusetts & 307 & 112 & 100.0 & 100.0 & .51 & .56 & 153 & 163 \\
\hline 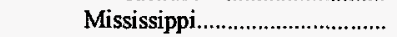 & 65 & - & - & - & .60 & - & 141 & - \\
\hline 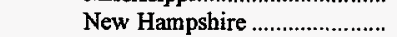 & 148 & 127 & - & - & .51 & .57 & 147 & 162 \\
\hline 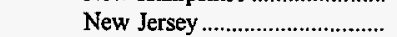 & 39 & - & - & - & .52 & - & 155 & - \\
\hline New York & 227 & 158 & 100.0 & 100.0 & .47 & .49 & 170 & 174 \\
\hline Texas & 9 & - & - & - & .54 & - & 186 & - \\
\hline 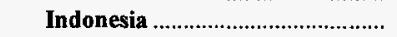 & 297 & 287 & 75.4 & 100.0 & .26 & .38 & 159 & 162 \\
\hline 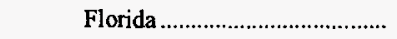 & 297 & 287 & 75.4 & 100.0 & .26 & .38 & 159 & 162 \\
\hline 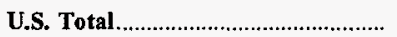 & 452,348 & 428,751 & 81.3 & 82.8 & 1.06 & 1.08 & 126 & 129 \\
\hline
\end{tabular}

* For quantity data, the number is less than 0.5 thousand short tons. For Contract Receipts (percent), the value is less than 0.05 .

Notes: Total may not equal sum of components because of independent rounding. MM Btu represents million Btu.

Source: Federal Energy Regulatory Commission, FERC Form 423, "Monthly Report of Cost and Quality of Fuels for Electric Plants." 
Table 30. Coal Receipts at Coke Plants by Census Division (Thousand Short Tons)

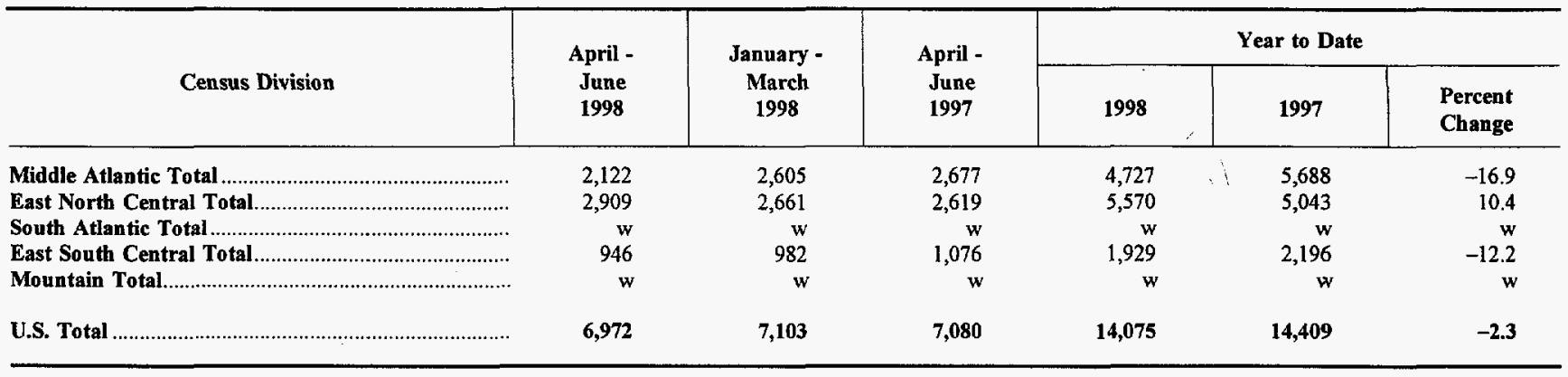

withheld to avoid disclosure of individual company data.

Notes: Total may not equal sum of components because of independent rounding.

Source: Energy Information Administration, Form EIA-5, "Coke Plant Report - Quarterly."

Table 31.

Avfogalfimeipts at Coke Plants by Census Division

(Dollars per Short Tons)

\begin{tabular}{|c|c|c|c|c|c|c|}
\hline \multirow[b]{2}{*}{ Census Division } & \multirow{2}{*}{$\begin{array}{c}\text { April - } \\
\text { June } \\
1998\end{array}$} & \multirow{2}{*}{$\begin{array}{c}\text { January - } \\
\text { March } \\
1998\end{array}$} & \multirow{2}{*}{$\begin{array}{c}\text { April - } \\
\text { June } \\
1997\end{array}$} & \multicolumn{3}{|c|}{ Year to Date } \\
\hline & & & & 1998 & 1997 & $\begin{array}{l}\text { Percent } \\
\text { Change }\end{array}$ \\
\hline Middle Atlantic Total & $\$ 43.93$ & $\$ 45.60$ & $\$ 47.36$ & $\$ 44.85$ & $\$ 47.61$ & -5.8 \\
\hline 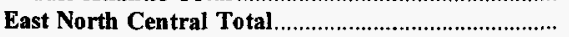 & 48.87 & 48.51 & 50.10 & 48.70 & 50.24 & -3.1 \\
\hline 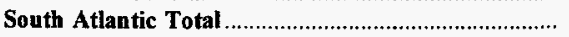 & w & w & w & $\mathbf{w}$ & w & w \\
\hline 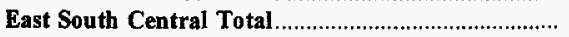 & $\$ 47.05$ & $\$ 47.73$ & $\$ 47.66$ & $\$ 47.40$ & $\$ 47.72$ & -.7 \\
\hline Mountain Total & w & w & w & w & $\mathbf{w}$ & w \\
\hline 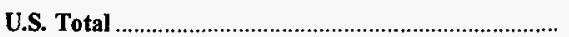 & $\$ 46.05$ & $\$ 46.62$ & $\$ 48.24$ & $\$ 46.34$ & $\$ 48.20$ & -3.9 \\
\hline
\end{tabular}

1 Based on the cost including insurance and freight (c.i.f. cost).

Withheld to avoid disclosure of individual company data.

Notes: Total may not equal sum of components because of independent rounding.

Source: Energy Information Administration, Form EIA-5, "Coke Plant Report - Quarterly." 
Table 32. Coal Receipts at Other Industrial Plants by Census Division and State (Thousand Short Tons)

\begin{tabular}{|c|c|c|c|c|c|c|}
\hline \multirow{2}{*}{$\begin{array}{c}\text { Census Division } \\
\text { and State }\end{array}$} & \multirow{2}{*}{$\begin{array}{l}\text { April - } \\
\text { June } \\
1998\end{array}$} & \multirow{2}{*}{$\begin{array}{c}\text { January - } \\
\text { March } \\
1998\end{array}$} & \multirow{2}{*}{$\begin{array}{c}\text { April - } \\
\text { June } \\
1997\end{array}$} & \multicolumn{3}{|c|}{ Year to Date } \\
\hline & & & & 1998 & 1997 & $\begin{array}{l}\text { Percent } \\
\text { Change }\end{array}$ \\
\hline New England Total & $\mathbf{w}$ & 72 & 64 & $\mathbf{w}$ & 113 & $\mathbf{w}$ \\
\hline 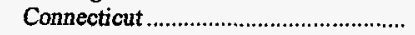 & - & - & - & - & - & - \\
\hline 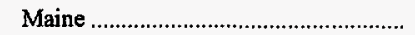 & $\mathrm{w}$ & $\mathbf{w}$ & w & $\mathbf{w}$ & $\mathbf{w}$ & $\mathrm{w}$ \\
\hline 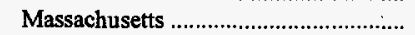 & $\mathrm{w}$ & $\mathbf{w}$ & $\mathrm{w}$ & $\mathrm{w}$ & w & $\mathrm{w}$ \\
\hline 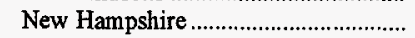 & 50 & - & - & 50 & - & - \\
\hline Rhode Island.............................................. & - & - & - & - & - & - \\
\hline Vermont & 27 & 27 & - & 54 & - & - \\
\hline Middle Atlantic Total & 1,442 & $\mathbf{w}$ & $\mathbf{w}$ & 2,794 & $\mathbf{w}$ & $\mathbf{w}$ \\
\hline New Jersey ............................................ & w & $\mathbf{w}$ & $\mathbf{w}$ & $\mathrm{w}$ & $\mathbf{w}$ & w \\
\hline New York & $\mathrm{w}$ & 285 & 369 & w & 643 & w \\
\hline Pennsylvania ............................................... & 1,046 & 1,064 & 1,099 & 2,110 & 2,136 & -1.2 \\
\hline East North Central Total ......................... & 3,858 & $\mathbf{3 , 8 3 8}$ & 4,208 & 7,695 & 8,244 & -6.7 \\
\hline Illinois & 984 & 1,027 & 938 & 2,011 & 1,935 & 3.9 \\
\hline Indiana & 1,143 & 1,145 & 1,278 & 2,288 & 2,469 & -7.3 \\
\hline Michigan ................................................. & 468 & 290 & 605 & 758 & 976 & -22.4 \\
\hline 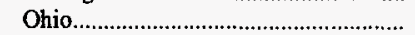 & 891 & 1,020 & 939 & 1,911 & 2,052 & -6.9 \\
\hline Wisconsin . & 372 & 355 & 448 & 727 & 812 & -10.5 \\
\hline West North Central Total ......................... & $\mathbf{3 , 2 8 7}$ & 3,016 & $\mathbf{3 , 1 3 2}$ & 6,302 & 6,247 & .9 \\
\hline 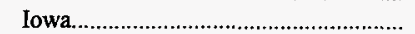 & 865 & 581 & 756 & 1,445 & 1,368 & 5.7 \\
\hline 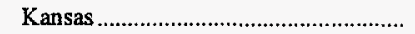 & 28 & 32 & 33 & 60 & 70 & -15.1 \\
\hline Minnesota & 397 & 343 & 323 & 740 & 685 & 8.1 \\
\hline Missouri ………………................... & 287 & 294 & 296 & 581 & 628 & -7.5 \\
\hline 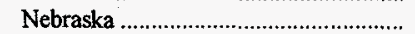 & $\mathrm{w}$ & w & w & $\mathbf{w}$ & $\mathbf{w}$ & w \\
\hline North Dakota .......................................... & $\mathrm{w}$ & $\mathrm{w}$ & w & $\mathbf{w}$ & $\mathbf{w}$ & w \\
\hline South Dakota .............. & 112 & 98 & 107 & 210 & 213 & -1.5 \\
\hline South Atlantic Total & $\mathbf{w}$ & $\mathbf{w}$ & $\mathbf{w}$ & $\mathbf{w}$ & $\mathbf{w}$ & $\mathbf{w}$ \\
\hline 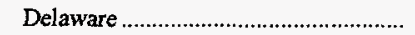 & $w$ & $w$ & $w$ & $w$ & $\mathbf{w}$ & $w$ \\
\hline District of Columbia ................................. & - & - & - & - & - & - \\
\hline 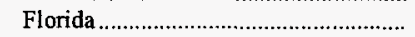 & 356 & 333 & 342 & 689 & 678 & 1.6 \\
\hline Georgia & 476 & 562 & 474 & 1,038 & 1,014 & 2.5 \\
\hline Maryland ................................................. & 174 & 190 & 197 & 365 & 390 & -6.6 \\
\hline North Carolina ........................................... & 496 & 563 & 520 & 1,060 & 1,169 & -9.3 \\
\hline 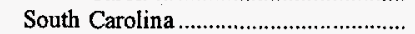 & 499 & 482 & 481 & 981 & 1,018 & -3.6 \\
\hline Virginia & 537 & 642 & 638 & 1,179 & 1,329 & -11.3 \\
\hline 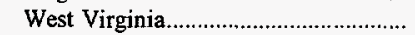 & 370 & 413 & 391 & 783 & 815 & -3.9 \\
\hline East South Central Total....................... & $\mathbf{w}$ & $\mathbf{w}$ & $\mathbf{w}$ & w & $\mathbf{w}$ & $\mathbf{w}$ \\
\hline Alabama & 655 & 710 & 659 & 1,365 & 1,306 & 4.5 \\
\hline 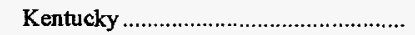 & 449 & 497 & 587 & 945 & 1,162 & -18.6 \\
\hline 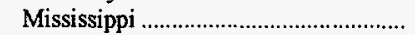 & $\mathrm{w}$ & w & w & $\mathbf{w}$ & w & $\mathbf{w}$ \\
\hline 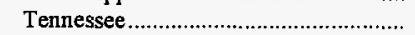 & 917 & 963 & 900 & 1,880 & 1,846 & 1.8 \\
\hline West South Central Total........................ & 1,522 & 1,476 & 1,361 & 2,999 & $\mathbf{2 , 8 0 8}$ & 6.8 \\
\hline 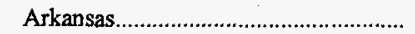 & 73 & 70 & 75 & 142 & 167 & -15.1 \\
\hline 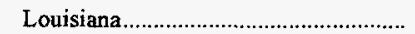 & $\mathbf{w}$ & $\mathbf{w}$ & $\mathbf{w}$ & $\mathbf{w}$ & w & $\mathbf{w}$ \\
\hline Oklahoma & $\mathrm{w}$ & $\mathbf{w}$ & $\mathbf{w}$ & $\mathbf{w}$ & $\mathbf{w}$ & w \\
\hline Texas & 1,250 & 1,167 & 1,109 & 2,418 & 2,259 & 7.0 \\
\hline Mountain Total .......................................... & 1,204 & 1,218 & 1,165 & 2,422 & 2,334 & 3.8 \\
\hline Arizona & 195 & 174 & 151 & 368 & 339 & 8.6 \\
\hline Colorado & 158 & 180 & 195 & 338 & 386 & -12.5 \\
\hline Idaho & 67 & $\mathbf{w}$ & $\mathbf{w}$ & 175 & w & $\mathbf{w}$ \\
\hline 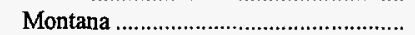 & $\mathrm{w}$ & $\mathrm{w}$ & $\mathbf{w}$ & $\mathbf{w}$ & w & w \\
\hline Nevada & $\mathrm{w}$ & w & $\mathbf{w}$ & $\mathbf{w}$ & w & $\mathbf{w}$ \\
\hline 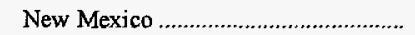 & $\mathrm{w}$ & $\mathbf{w}$ & w & w & $\mathrm{w}$ & w \\
\hline 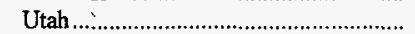 & 249 & 139 & 198 & 388 & 262 & 48.2 \\
\hline 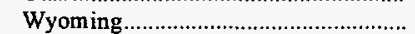 & 454 & 523 & 478 & 977 & 967 & 1.1 \\
\hline 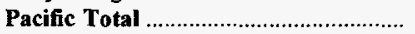 & 2,171 & 807 & 599 & 2,978 & 1,189 & 150.4 \\
\hline Alaska & $\mathbf{w}$ & $\mathbf{w}$ & $\mathbf{w}$ & $\mathbf{w}$ & w & w \\
\hline 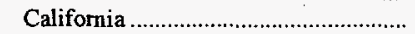 & 677 & 692 & 522 & 1,369 & 996 & 37.4 \\
\hline Hawaii ........................................................ & $\mathbf{w}$ & $\mathbf{w}$ & $\mathbf{w}$ & w & $\mathbf{w}$ & w \\
\hline 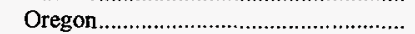 & $\mathrm{w}$ & $\mathbf{w}$ & $\mathbf{w}$ & $\mathrm{w}$ & $\mathbf{w}$ & $\mathbf{w}$ \\
\hline Washington & w & 14 & 44 & $w$ & 91 & w \\
\hline U.S. Total & 18,614 & 17,213 & 17,280 & 35,827 & 34,632 & 3.5 \\
\hline
\end{tabular}

witheld to avoid disclosure of individual company data.

Note: Total may not equal sum of components because of independent rounding.

Sources: Energy Information Administration, Form EIA-3, "Quarterly Coal Consumption-Manufacturing Plants;" Form ELA-867, "Annual Nonutility Power Producer Report;"' and Form ELA-7A, "Coal Production Report." 
Table 33. Average Price of Coal Receipts at Other Industrial Plants by Census Division and State (Dollars per Short Ton)

\begin{tabular}{|c|c|c|c|c|c|c|}
\hline \multirow{2}{*}{$\begin{array}{l}\text { Census Division } \\
\text { and State }\end{array}$} & \multirow{2}{*}{$\begin{array}{l}\text { April - June } \\
1998\end{array}$} & \multirow{2}{*}{$\begin{array}{c}\text { January - March } \\
1998\end{array}$} & \multirow{2}{*}{$\begin{array}{c}\text { April - June } \\
1997\end{array}$} & \multicolumn{3}{|c|}{ Year to Date } \\
\hline & & & & 1998 & 1997 & $\begin{array}{l}\text { Percent } \\
\text { Change }\end{array}$ \\
\hline New England Total.................................. & $\mathbf{w}$ & $\$ 64.83$ & $\$ 59.29$ & $\mathbf{w}$ & $\$ 62.04$ & $\mathbf{w}$ \\
\hline 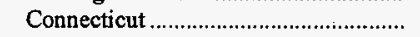 & - & - & - & - & - & - \\
\hline Maine & w & $\mathbf{w}$ & $\mathbf{w}$ & $\mathbf{w}$ & $\mathbf{w}$ & $\mathbf{w}$ \\
\hline 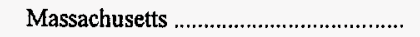 & w & $\mathbf{w}$ & w & $\mathbf{w}$ & $\mathbf{w}$ & $\mathbf{w}$ \\
\hline New Hampshire ........................................ & - & - & - & - & - & - \\
\hline 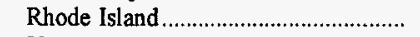 & - & - & - & - & - & - \\
\hline Vermont & - & - & - & - & - & - \\
\hline Middle Atlantic Total.............................. & $\$ 35.70$ & $\mathbf{w}$ & $\mathbf{w}$ & $\$ 35.49$ & $\mathbf{w}$ & $\mathbf{w}$ \\
\hline New Jersey & w & $w$ & $w$ & w & $w$ & w \\
\hline 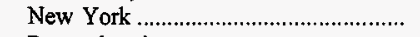 & w & $\$ 40.80$ & $\$ 41.24$ & $\mathbf{w}$ & $\$ 41.04$ & w \\
\hline 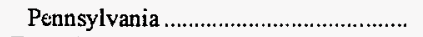 & $\$ 33.52$ & 33.98 & 33.69 & $\$ 33.75$ & 34.48 & -2.1 \\
\hline East North Central Total ....................... & .32 .54 & 32.80 & 33.04 & 32.67 & 33.04 & -1.1 \\
\hline Illinois & 29.05 & 29.19 & 29.52 & 29.12 & 29.51 & -1.3 \\
\hline Indiana & 30.03 & 30.12 & 28.85 & 30.08 & 29.01 & 3.7 \\
\hline Michigan & 40.01 & 42.30 & 40.73 & 40.86 & 41.54 & -1.6 \\
\hline 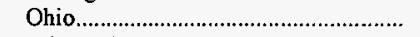 & 32.68 & 34.19 & 34.52 & 33.49 & 34.55 & -3.1 \\
\hline Wisconsin & 40.08 & 40.73 & 39.43 & 40.40 & 40.31 & .2 \\
\hline West North Central Total ...................... & 19.09 & 18.45 & 19.03 & 18.78 & 18.93 & -.8 \\
\hline lowa. & 29.41 & 24.99 & 30.18 & 27.68 & 28.03 & -1.2 \\
\hline 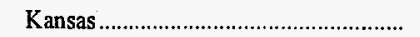 & 29.05 & 30.77 & 32.23 & 29.97 & 32.97 & -9.1 \\
\hline 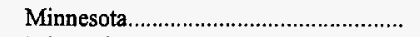 & 29.31 & 34.84 & 31.40 & 31.78 & 32.80 & -3.1 \\
\hline Missouri & 30.49 & 31.97 & 30.10 & 31.24 & 30.45 & 2.6 \\
\hline Nebraska & $w$ & $\mathbf{w}$ & w & $w$ & $w$ & $w$ \\
\hline 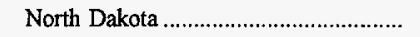 & $w$ & w & $\mathbf{w}$ & $\mathbf{w}$ & $\mathbf{w}$ & $\mathbf{w}$ \\
\hline 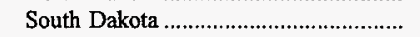 & $\$ 23.17$ & $\$ 24.99$ & $\$ 23.20$ & $\$ 24.02$ & $\$ 23.66$ & 1.5 \\
\hline South Atlantic Total................................. & $\mathbf{w}$ & $\mathbf{w}$ & $\mathbf{w}$ & $\mathbf{w}$ & $\mathbf{w}$ & $\mathbf{w}$ \\
\hline Delaware & $\mathbf{w}$ & w & $\mathbf{w}$ & $w$ & $w$ & $w$ \\
\hline District of Columbia & - & - & - & - & - & - \\
\hline 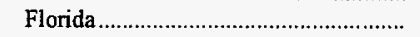 & $\$ 45.02$ & $\$ 44.69$ & $\$ 45.61$ & $\$ 44.86$ & $\$ 45.35$ & -1.1 \\
\hline 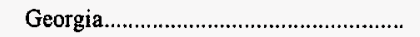 & 44.31 & 44.42 & 44.99 & 44.37 & 45.00 & -1.4 \\
\hline 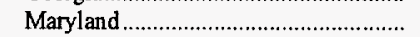 & 33.29 & 32.70 & 32.15 & 32.98 & 32.57 & 1.2 \\
\hline 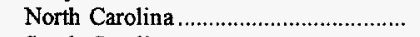 & 42.16 & 43.46 & 42.84 & 42.85 & 43.18 & -.8 \\
\hline 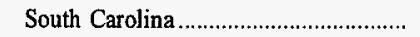 & 44.04 & 44.71 & 44.30 & 44.37 & 44.23 & .3 \\
\hline Virginia & 44.39 & 44.01 & 43.62 & 44.18 & 44.03 & .4 \\
\hline 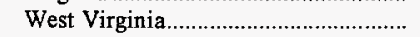 & 48.55 & 46.37 & 34.17 & 47.39 & 33.97 & 39.5 \\
\hline East South Central Total....................... & $\mathbf{w}$ & $\mathbf{w}$ & $\mathbf{w}$ & $\mathbf{w}$ & $\mathbf{w}$ & $\mathbf{w}$ \\
\hline Alabama & $\$ 37.53$ & $\$ 40.59$ & $\$ 39.91$ & $\$ 39.13$ & $\$ 40.16$ & -2.6 \\
\hline 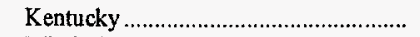 & 44.64 & 43.75 & 44.67 & 44.16 & 44.79 & -1.4 \\
\hline 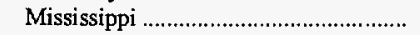 & w & w & w & $w$ & w & $w$ \\
\hline 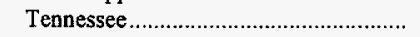 & $\$ 36.16$ & $\$ 37.27$ & $\$ 36.15$ & $\$ 36.73$ & $\$ 36.17$ & 1.6 \\
\hline West South Central Total...................... & 22.46 & 22.53 & 22.62 & $\mathbf{2 2 . 5 0}$ & 22.91 & -1.8 \\
\hline 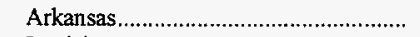 & 41.27 & 41.34 & 43.49 & 41.30 & 43.49 & -5.0 \\
\hline 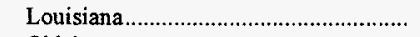 & $w$ & w & w & $w$ & $w$ & w \\
\hline Oklahoma & $\mathrm{w}$ & $\mathbf{w}$ & w & $w$ & w & w \\
\hline 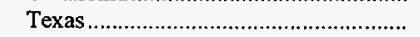 & $\$ 20.76$ & $\$ 20.41$ & $\$ 20.15$ & $\$ 20.59$ & $\$ 20.06$ & 2.6 \\
\hline 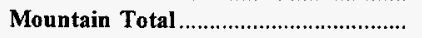 & 27.17 & 27.47 & 26.54 & 27.32 & 27.02 & 1.1 \\
\hline 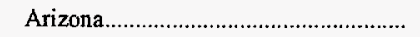 & 39.59 & 38.18 & 40.16 & 38.93 & 39.44 & -1.3 \\
\hline 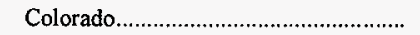 & 25.51 & 25.04 & 25.00 & 25.26 & 24.87 & 1.6 \\
\hline Idaho & 35.56 & $\mathbf{w}$ & w & 34.03 & w & $\mathrm{w}$ \\
\hline Montana & $\mathbf{w}$ & $\mathbf{w}$ & w & $\mathbf{w}$ & w & $w$ \\
\hline 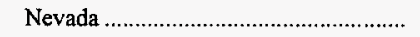 & $w$ & $\mathbf{w}$ & $\mathbf{w}$ & $\mathbf{w}$ & w & w \\
\hline 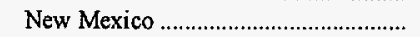 & $\mathbf{w}$ & $\mathbf{w}$ & $\mathbf{w}$ & $\mathbf{w}$ & w & w \\
\hline 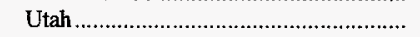 & $\$ 17.96$ & $\$ 20.04$ & $\$ 18.34$ & $\$ 18.60$ & $\$ 19.68$ & -5.5 \\
\hline 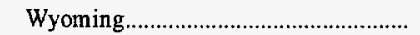 & 23.99 & 24.25 & 23.93 & 24.13 & 23.25 & 3.8 \\
\hline Pacific Total & 40.06 & 46.07 & 43.26 & 43.38 & 43.29 & .2 \\
\hline 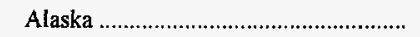 & w & $\mathbf{w}$ & $\mathbf{w}$ & w & $w$ & w \\
\hline 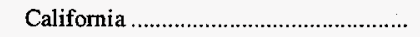 & $\$ 39.86$ & $\$ 42.86$ & $\$ 40.61$ & $\$ 41.38$ & $\$ 40.05$ & 3.3 \\
\hline Hawaii & w & $\mathbf{w}$ & w & $w$ & $\mathbf{w}$ & w \\
\hline Oregon & w & $\mathbf{w}$ & $w$ & $\mathrm{w}$ & $\mathbf{w}$ & $w$ \\
\hline Washington & $\mathbf{w}$ & $\$ 99.91$ & $\$ 55.87$ & $\mathbf{w}$ & $\$ 54.50$ & $w$ \\
\hline U.S. Total & $\$ 31.88$ & 32.77 & 32.29 & $\$ 32.32$ & 32.45 & -.4 \\
\hline
\end{tabular}

w Withheld to avoid disclosure of individual company data.

Note: Total may not equal sum of components because of independent rounding. Price data are for manufacturing plants only.

Sources: Energy Information Administration, Form EIA-3, "Quarterly Coal Consumption-Manufacturing Plants". 
Table 34. U.S. Coal Receipts at Manufacturing Plants by North American Industry Classification System (NAICS) Code

(Thousand Short Tons)

\begin{tabular}{|c|c|c|c|c|c|c|}
\hline \multirow[b]{2}{*}{ NAICS Code } & \multirow{2}{*}{$\begin{array}{l}\text { April - } \\
\text { June } \\
1998\end{array}$} & \multirow{2}{*}{$\begin{array}{c}\text { January - } \\
\text { March } \\
1998\end{array}$} & \multirow{2}{*}{$\begin{array}{c}\text { April - } \\
\text { June } \\
1997\end{array}$} & \multicolumn{3}{|c|}{ Year to Date } \\
\hline & & & & 1998 & 1997 & $\begin{array}{l}\text { Percent } \\
\text { Change }\end{array}$ \\
\hline 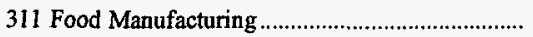 & 1,905 & 2,088 & 1,772 & 3,993 & 3,837 & 4.1 \\
\hline 312 Beverage and Tobacco Product Mfg.................... & 126 & 140 & 127 & 266 & 291 & -8.5 \\
\hline 313 Textile Mills & 206 & 263 & 240 & 469 & 506 & -7.4 \\
\hline 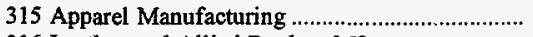 & $\mathbf{w}$ & $w$ & $w$ & $\mathbf{w}$ & $w$ & w \\
\hline 316 Leather and Allied Product Mfg........................... & - & $\mathbf{w}$ & $\mathbf{w}$ & - & $\mathbf{w}$ & - \\
\hline 321 Wood Product Manufacturing.............................. & $\mathbf{w}$ & w & $\mathbf{w}$ & $\mathbf{w}$ & $w$ & w \\
\hline 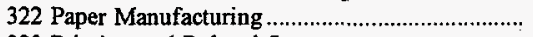 & 2,897 & 3,262 & 3,093 & 6,159 & 6,479 & -4.9 \\
\hline 323 Printing and Related Support................................. & - & - & - & - & - & - \\
\hline 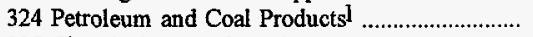 & $\mathbf{w}$ & 1,633 & 1,666 & $\mathbf{w}$ & 3,305 & w \\
\hline 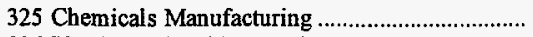 & 3,013 & 3,164 & 3,149 & 6,177 & 6,480 & -4.7 \\
\hline 326 Plastics and Rubber Products Mfg ...................... & 43 & 51 & 41 & 94 & 109 & -13.4 \\
\hline 327 Nonmetallic Mineral Products Mfg ........................ & $\mathbf{w}$ & $\mathbf{w}$ & $\mathbf{w}$ & $\mathbf{w}$ & $w$ & w \\
\hline 331 Primary Metal Manufacturing 2 & $\mathbf{w}$ & 1,914 & 2,240 & $\mathbf{w}$ & 4,032 & $\mathbf{w}$ \\
\hline 332 Fabricated Metal Product Mfg............................. & 39 & 81 & 52 & 120 & 161 & -25.6 \\
\hline 333 Machinery Manufacturing .................................... & 50 & 91 & 56 & 141 & 146 & -3.2 \\
\hline 334 Computer and Electronic Product Mfg .............. & w & $w$ & w & w & $w$ & w \\
\hline 335 Elec. Equip., Appl., Components Mfg ................. & $\mathbf{w}$ & w & $\mathbf{w}$ & w & $\mathbf{w}$ & w \\
\hline 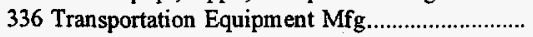 & 117 & 242 & 177 & 358 & 507 & -29.2 \\
\hline 337 Furniture and Related Product Mfg................... & w & $\mathbf{w}$ & w & w & $\mathbf{w}$ & w \\
\hline 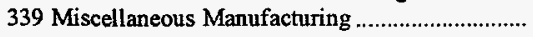 & $\mathbf{w}$ & $\mathbf{w}$ & w & $\mathbf{w}$ & $\mathbf{w}$ & w \\
\hline U.S. Total & 15,885 & 16,010 & 16,284 & 31,895 & 32,606 & -2.2 \\
\hline
\end{tabular}

1 Includes coal gasification projects.

2 Excludes coke plants.

w Withheld to avoid disclosure of individual company data.

Note: Total may not equal sum of components because of independent rounding.

Source: Energy Information Administration, Form EIA-3, "Quarterly Coal Consumption Report - Manufacturing Plants."

Table 35. Average Price of U.S. Coal Receipts at Manufacturing Plants by North American Industry Classification System (NAICS) Code (Dollars per Short Ton)

\begin{tabular}{|c|c|c|c|c|}
\hline NAICS Code & $\begin{array}{c}\text { April - June } \\
1998\end{array}$ & $\begin{array}{c}\text { January - March } \\
1998\end{array}$ & $\begin{array}{c}\text { April - June } \\
1997\end{array}$ & $\begin{array}{c}\text { Percent Difference } \\
\text { April - June: } \\
1998 \text { versus } 1997\end{array}$ \\
\hline 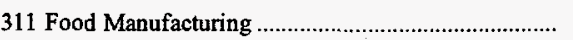 & $\$ 28.92$ & $\$ 29.58$ & $\$ 30.35$ & -4.7 \\
\hline 312 Beverage and Tobacco Product Mfg ............................ & 46.49 & 46.27 & 45.97 & 1.1 \\
\hline 313 Textile Mills & 47.97 & 47.53 & 47.73 & .5 \\
\hline 315 Apparel Manufacturing & $\mathbf{w}$ & $\mathbf{w}$ & w & $\mathbf{w}$ \\
\hline 316 Leather and Allied Product Mfg & - & $\mathbf{w}$ & $\mathbf{w}$ & - \\
\hline 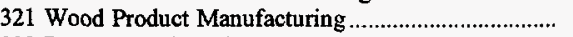 & $\mathbf{w}$ & $\mathbf{w}$ & $\mathbf{w}$ & $\mathbf{w}$ \\
\hline 322 Paper Manufacturing & $\$ 39.39$ & $\$ 39.57$ & $\$ 39.67$ & -.7 \\
\hline 323 Printing and Related Support & - & - & - & - \\
\hline 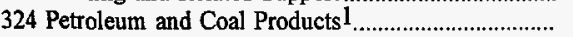 & w & 12.20 & 11.45 & $w$ \\
\hline 325 Chemicals Manufacturing & $\$ 36.42$ & 35.85 & 34.87 & 4.4 \\
\hline 326 Plastics and Rubber Products Mfg................................. & 31.46 & 32.71 & 27.63 & 13.9 \\
\hline 327 Nonmetallic Mineral Products Mfg & $w$ & $\mathbf{w}$ & $\mathbf{w}$ & w \\
\hline 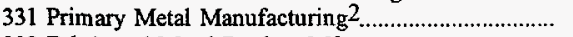 & w & $\$ 25.76$ & $\$ 25.93$ & $w$ \\
\hline 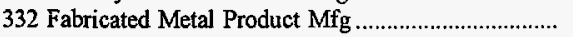 & $\$ 45.90$ & 47.14 & 46.24 & -.7 \\
\hline 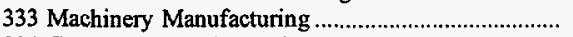 & 39.23 & 39.07 & 37.47 & 4.7 \\
\hline 334 Computer and Electronic Product Mfg..................... & $\mathbf{w}$ & w & $\mathbf{w}$ & $w$ \\
\hline 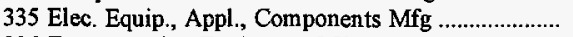 & w & $\mathbf{w}$ & w & w \\
\hline 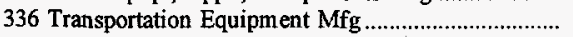 & $\$ 38.89$ & $\$ 41.66$ & $\$ 41.86$ & -7.1 \\
\hline 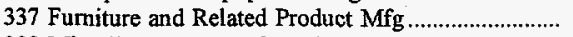 & $\mathbf{w}$ & w & $\mathbf{w}$ & w \\
\hline 339 Miscellaneous Manufacturing .................................. & w & w & $\mathbf{w}$ & $w$ \\
\hline U.S. Total & $\$ 31.88$ & $\$ 32.77$ & $\$ 32.29$ & -1.3 \\
\hline
\end{tabular}

1 Includes coal gasification projects.

2 Excludes coke plants.

witheld to avoid disclosure of individual company data.

Note: Total may not equal sum of components because of independent rounding.

Source: Energy Information Administration, Form EIA-3, "Quarterly Coal Consumption Report - Manufacturing Plants." 
Table 36. Coal Receipts by the Residential and Commercial Sector by Census Division and State (Thousand Short Tons)

\begin{tabular}{|c|c|c|c|c|c|c|}
\hline \multirow{2}{*}{$\begin{array}{c}\text { Census Division } \\
\text { and State }\end{array}$} & \multirow{2}{*}{$\begin{array}{l}\text { April - } \\
\text { June } \\
1998\end{array}$} & \multirow{2}{*}{$\begin{array}{c}\text { January - } \\
\text { March } \\
1998\end{array}$} & \multirow{2}{*}{$\begin{array}{l}\text { April - } \\
\text { June } \\
1997\end{array}$} & \multicolumn{3}{|c|}{ Year to Date } \\
\hline & & & & 1998 & 1997 & $\begin{array}{l}\text { Percent } \\
\text { Change }\end{array}$ \\
\hline 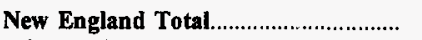 & 10 & 15 & 10 & 26 & 26 & 0.0 \\
\hline 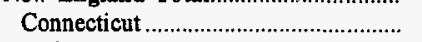 & $w$ & $\mathrm{w}$ & $\mathbf{w}$ & $\mathbf{w}$ & $\mathbf{w}$ & $\mathbf{w}$ \\
\hline 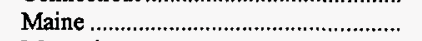 & $w$ & $w$ & $\mathbf{w}$ & $\mathbf{w}$ & $\mathbf{w}$ & w \\
\hline 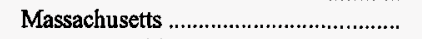 & $w$ & $\mathbf{w}$ & $\mathbf{w}$ & w & $\mathbf{w}$ & $\mathbf{w}$ \\
\hline New Hampshire & $\mathbf{w}$ & $\mathbf{w}$ & $\mathbf{w}$ & $\mathbf{w}$ & $\mathbf{w}$ & $w$ \\
\hline 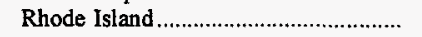 & $w$ & $\mathbf{w}$ & $\mathbf{w}$ & $\mathbf{w}$ & $w$ & $\mathbf{w}$ \\
\hline Vermont & $\mathbf{w}$ & w & w & $\mathbf{w}$ & $w$ & $\mathbf{w}$ \\
\hline Middle Atlantic Total ................................ & 301 & 451 & 301 & 752 & 752 & .0 \\
\hline 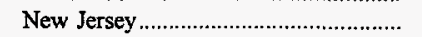 & $\mathbf{w}$ & $\mathbf{w}$ & $\mathbf{w}$ & $\mathbf{w}$ & $\mathbf{w}$ & $\mathbf{w}$ \\
\hline New York & $\mathbf{w}$ & $\mathbf{w}$ & $\mathbf{w}$ & $\mathbf{w}$ & $\mathbf{w}$ & $\mathbf{w}$ \\
\hline 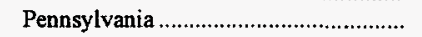 & 249 & 373 & 249 & 622 & 622 & .0 \\
\hline East North Central Total ........................ & 274 & 411 & 274 & 685 & 685 & .0 \\
\hline 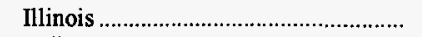 & $\mathbf{w}$ & w & w & $\mathbf{w}$ & $\mathbf{w}$ & $w$ \\
\hline 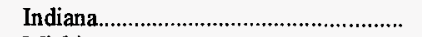 & 79 & 119 & 79 & 198 & 198 & .0 \\
\hline 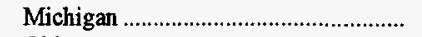 & $\mathbf{w}$ & $\mathrm{w}$ & $\mathbf{w}$ & $w$ & $\mathbf{w}$ & $\mathbf{w}$ \\
\hline 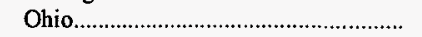 & 66 & 99 & 66 & 164 & 164 & .0 \\
\hline Wisconsin & w & $w$ & $\mathbf{w}$ & w & w & $w$ \\
\hline West North Central Total .................... & $\mathbf{w}$ & $\mathbf{w}$ & $\mathbf{w}$ & $\mathbf{w}$ & $\mathbf{w}$ & $\mathbf{w}$ \\
\hline 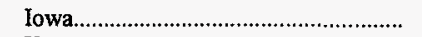 & 75 & 112 & 75 & 187 & 187 & .0 \\
\hline 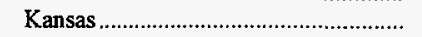 & $*$ & 1 & $*$ & 1 & 1 & .0 \\
\hline 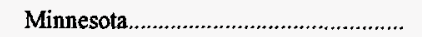 & 21 & 32 & 21 & 53 & 53 & .0 \\
\hline 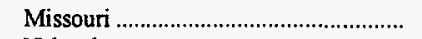 & w & w & w & $\mathbf{w}$ & $\mathbf{w}$ & $\mathbf{w}$ \\
\hline 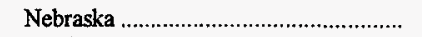 & $\mathbf{w}$ & $\mathbf{w}$ & $w$ & $\mathbf{w}$ & $\mathbf{w}$ & $w$ \\
\hline North Dakota & $\mathbf{w}$ & $\mathbf{w}$ & $\mathbf{w}$ & $w$ & $\mathbf{w}$ & $\mathbf{w}$ \\
\hline 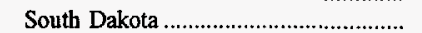 & $w$ & $\mathbf{w}$ & $w$ & w & $\mathbf{w}$ & $\mathbf{w}$ \\
\hline 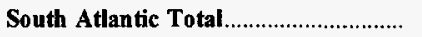 & 120 & 180 & 120 & 299 & 299 & .0 \\
\hline 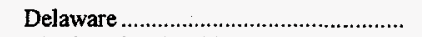 & w & w & $\mathbf{w}$ & $w$ & w & w \\
\hline 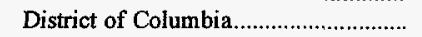 & 8 & 12 & 8 & 20 & 20 & .0 \\
\hline Florida & - & - & - & - & - & - \\
\hline 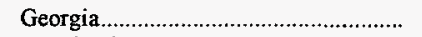 & 3 & 5 & 3 & 8 & 8 & .0 \\
\hline 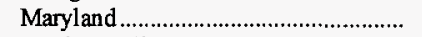 & $\mathbf{w}$ & w & $\mathbf{w}$ & $\mathbf{w}$ & $w$ & $w$ \\
\hline 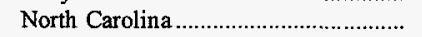 & 38 & 58 & 38 & 96 & 96 & 0 \\
\hline 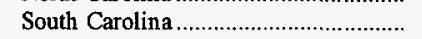 & $*$ & $*$ & $\cdot$ & $*$ & $*$ & .0 \\
\hline 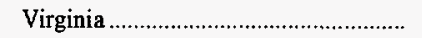 & $w$ & w & $w$ & $\mathbf{w}$ & $\mathbf{w}$ & w \\
\hline West Virginia & $\mathbf{w}$ & $\mathbf{w}$ & $\mathbf{w}$ & $\mathbf{w}$ & $w$ & $\mathbf{w}$ \\
\hline East South Central Total...................... & 111 & 167 & 111 & 279 & 279 & .0 \\
\hline 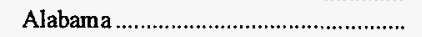 & 15 & 22 & 15 & 36 & 36 & .0 \\
\hline 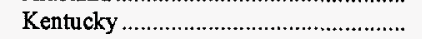 & $\mathbf{w}$ & $\mathbf{w}$ & $\mathbf{w}$ & $w$ & $\mathbf{w}$ & $\mathbf{w}$ \\
\hline 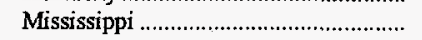 & $\mathbf{w}$ & $w$ & $\mathbf{w}$ & $w$ & $\mathbf{w}$ & $\mathbf{w}$ \\
\hline Tennessee & w & $\mathbf{w}$ & $w$ & $\mathbf{w}$ & w & $w$ \\
\hline West South Central Total....................... & 58 & 87 & 58 & 145 & 145 & .0 \\
\hline 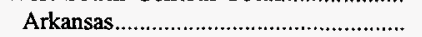 & $*$ & $*$ & $*$ & $*$ & $*$ & .0 \\
\hline 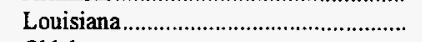 & $\mathbf{w}$ & w & $\mathbf{w}$ & $\mathbf{w}$ & $\mathbf{w}$ & $\mathbf{w}$ \\
\hline Oklahoma & $\mathbf{w}$ & $\mathbf{w}$ & $\mathbf{w}$ & $\mathbf{w}$ & $\mathbf{w}$ & $w$ \\
\hline Texas & $*$ & $*$ & $*$ & * & $*$ & .0 \\
\hline 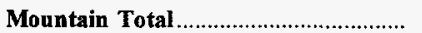 & $\mathbf{w}$ & $\mathbf{w}$ & $\mathbf{w}$ & $\mathbf{w}$ & $\mathbf{w}$ & $\mathbf{w}$ \\
\hline 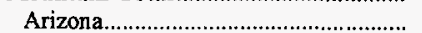 & * & $*$ & $*$ & $*$ & $*$ & .0 \\
\hline Colorado & 13 & 19 & 13 & 32 & 32 & .0 \\
\hline Idaho & 6 & 9 & 6 & 15 & 15 & 0 \\
\hline Montana & $\mathbf{w}$ & $\mathbf{w}$ & $\mathbf{w}$ & $\mathbf{w}$ & $\mathbf{w}$ & w \\
\hline Nevada & $w$ & $\mathbf{w}$ & $w$ & $w$ & $\mathbf{w}$ & $\mathbf{w}$ \\
\hline 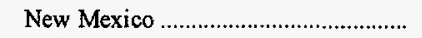 & $w$ & $w$ & $\mathbf{w}$ & $\mathbf{w}$ & $\mathbf{w}$ & $w$ \\
\hline 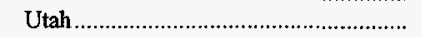 & $\mathbf{w}$ & $\mathbf{w}$ & $\mathbf{w}$ & $\mathbf{w}$ & $\mathbf{w}$ & w \\
\hline Wyoming & 28 & 42 & 28 & 70 & 70 & .0 \\
\hline 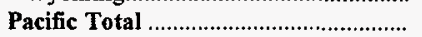 & 127 & 190 & 127 & 317 & 317 & .0 \\
\hline Alaska & 101 & 151 & 101 & 251 & 251 & .0 \\
\hline 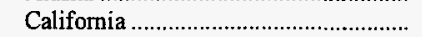 & 22 & 33 & 22 & 54 & 54 & .0 \\
\hline 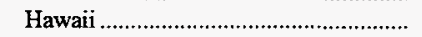 & - & - & - & - & - & - \\
\hline 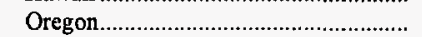 & * & * & $*$ & $*$ & * & .0 \\
\hline 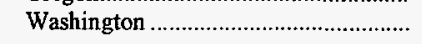 & 4 & 6 & 4 & 11 & 11 & .0 \\
\hline 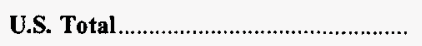 & 1,293 & 1,939 & 1,293 & 3,232 & 3,232 & .0 \\
\hline
\end{tabular}

* Rounded to zero.

withheld to avoid disclosure of individual company data.

Note: Total may not equal sum of components because of independent rounding.

Source: Energy Information Administration, Form EIA-6, "Coal Distribution Report." 


\section{Consumption}


Table 37. U.S. Coal Consumption by End-Use Sector 1992-1998

(Thousand Short Tons)

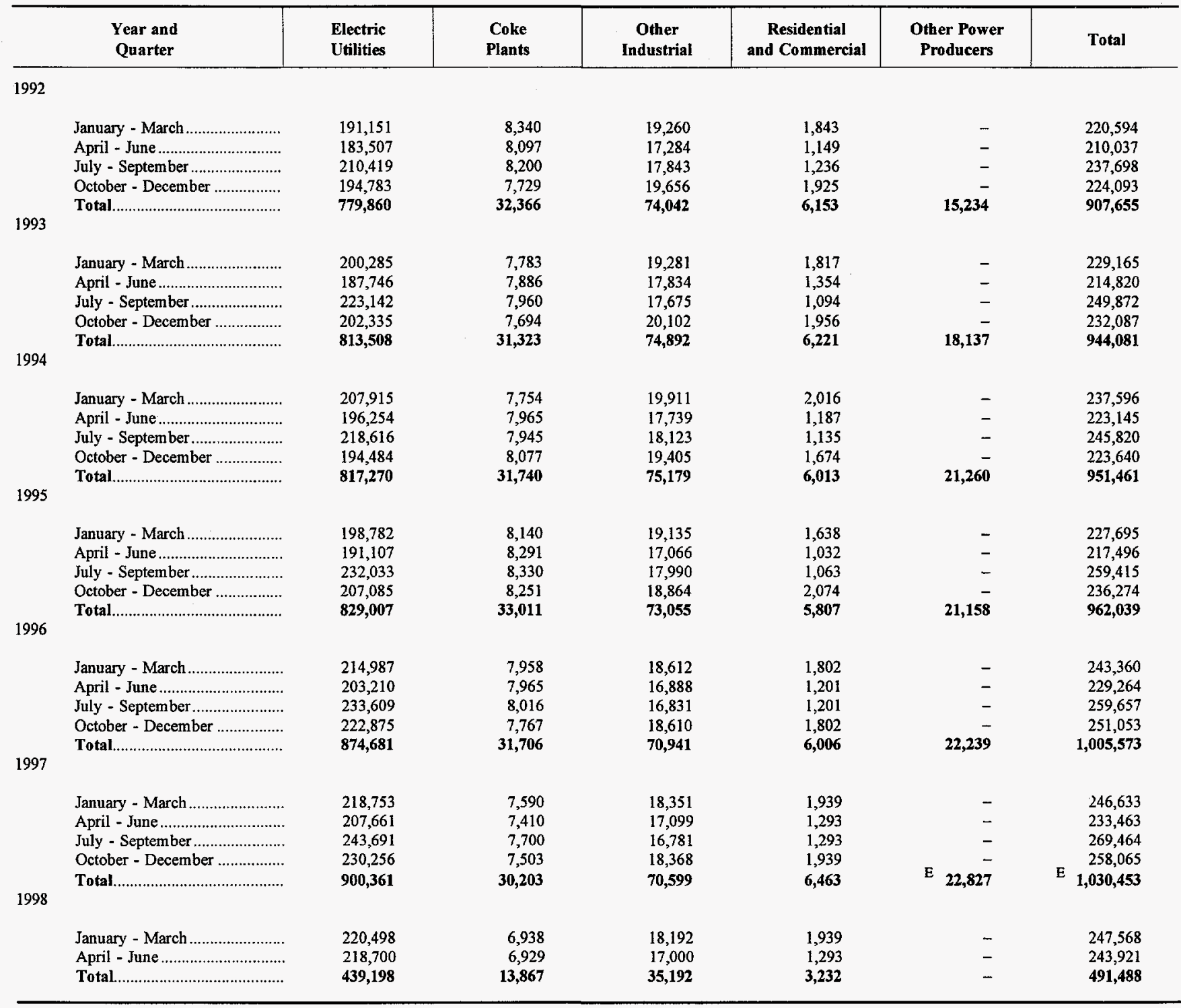

\footnotetext{
E Estimated.
}

Notes: Total may not equal sum of components because of independent rounding.

Sources: Energy Information Administration (EIA) - Electric Utilities: Form EIA-759, "Monthly Power Plant Report" • Coke Plants: Form EIA-5,

"Coke Plant Report - Quarterly" - Other Industrial; Form EIA-3, "Quarterly Coal Consumption Report - Manufacturing Plants;" Form EIA-867, "Annual Nonutility Power Producer Report," and Form EIA-7A, "Coal Production Report." - Residential and Commercial: Form EIA-6, "Coal Distribution Report." 
Table 38. Coal Consumption by Census Division and State (Thousand Short Tons)

\begin{tabular}{|c|c|c|c|c|c|c|}
\hline \multirow{2}{*}{$\begin{array}{c}\text { Census Division } \\
\text { and State }\end{array}$} & \multirow{2}{*}{$\begin{array}{l}\text { April - } \\
\text { June } \\
1998\end{array}$} & \multirow{2}{*}{$\begin{array}{c}\text { January - } \\
\text { March } \\
1998\end{array}$} & \multirow{2}{*}{$\begin{array}{l}\text { April - } \\
\text { June } \\
1997\end{array}$} & \multicolumn{3}{|c|}{ Year to Date } \\
\hline & & & & 1998 & 1997 & $\begin{array}{l}\text { Percent } \\
\text { Change }\end{array}$ \\
\hline New England Total.............. & 1,608 & 1,760 & 1,861 & 3,369 & 3,772 & -10.7 \\
\hline Connecticut & 137 & 210 & 262 & 347 & 555 & -37.6 \\
\hline 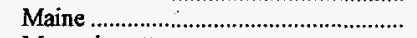 & 30 & 44 & 46 & 75 & 103 & -27.3 \\
\hline 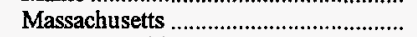 & 1,062 & 1,127 & 1,133 & 2,189 & 2,266 & -3.4 \\
\hline 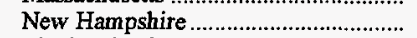 & 352 & 351 & 420 & 702 & 845 & -16.9 \\
\hline 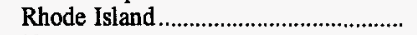 & 1 & 1 & 1 & 1 & 1 & .0 \\
\hline Vermont & 27 & 28 & 1 & 55 & 1 & $\mathrm{NM}$ \\
\hline Middle Atlantic Total ............................. & 17,036 & 18,240 & 16,636 & 35,276 & 34,982 & .8 \\
\hline 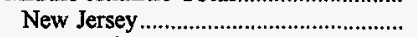 & 472 & 494 & 427 & 965 & 1,227 & -21.4 \\
\hline 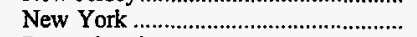 & 2,986 & 3,050 & 2,609 & 6,036 & 5,476 & 10.2 \\
\hline 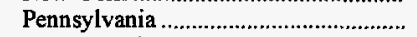 & 13,578 & 14,696 & 13,600 & 28,274 & 28,279 & * \\
\hline East North Central Total ..................... & $\mathbf{5 6 , 4 0 5}$ & $\mathbf{5 7 , 7 0 0}$ & 54,130 & 114,105 & 113,083 & .9 \\
\hline 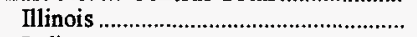 & 10,437 & 10,697 & 11,160 & 21,133 & 22,973 & -8.0 \\
\hline Indiana & 15,954 & 16,134 & 14,997 & 32,089 & 31,694 & 1.2 \\
\hline 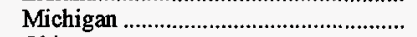 & 9,032 & 9,117 & 8,283 & 18,148 & 17,262 & 5.1 \\
\hline Ohio & 15,030 & 15,701 & 13,581 & 30,730 & 28,596 & 7.5 \\
\hline 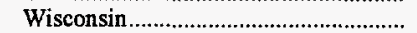 & 5,953 & 6,051 & 6,108 & 12,004 & 12,558 & -4.4 \\
\hline West North Central Total ..................... & 33,859 & 36,172 & 30,849 & 70,031 & 66,009 & 6.1 \\
\hline 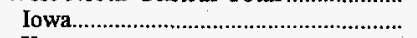 & 5,460 & 5,845 & 4,490 & 11,305 & 10,150 & 11.4 \\
\hline 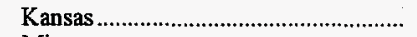 & 4,435 & 4,403 & 3,599 & 8,838 & 8,077 & 9.4 \\
\hline 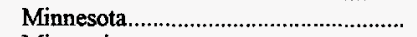 & 4,313 & 5,113 & 3,981 & 9,426 & 9,177 & 2.7 \\
\hline 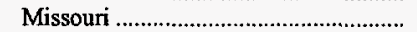 & 9,358 & 9,291 & 8,746 & 18,649 & 17,522 & 6.4 \\
\hline 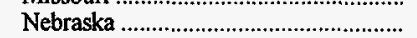 & 2,942 & 2,842 & 2,878 & 5,785 & 5,812 & -.5 \\
\hline 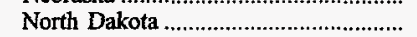 & 6,712 & 8,058 & 6,536 & 14,770 & 14,079 & 4.9 \\
\hline South Dakota & 639 & 620 & 619 & 1,259 & 1,192 & 5.6 \\
\hline 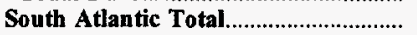 & 43,232 & 39,957 & 39,975 & 83,190 & 80,087 & 3.9 \\
\hline 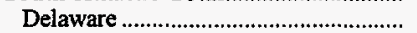 & 489 & 398 & 445 & 888 & 930 & -4.5 \\
\hline 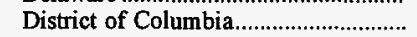 & 8 & 12 & 8 & 20 & 20 & .0 \\
\hline Florida & 7,111 & 6,500 & 7,121 & 13,611 & 13,750 & -1.0 \\
\hline 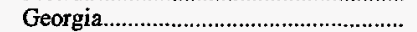 & 8,493 & 6,817 & 7,651 & 15,310 & 14,620 & 4.7 \\
\hline Maryland & 2,878 & 2,924 & 2,520 & 5,802 & 5,379 & 7.9 \\
\hline 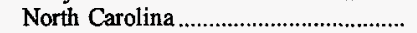 & 7,268 & 6,537 & 6,819 & 13,804 & 13,677 & .9 \\
\hline 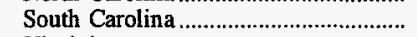 & 3,617 & 3,332 & 3,111 & 6,948 & 6,193 & 12.2 \\
\hline Virginia & 3,823 & 4,005 & 3,614 & 7,827 & 7,379 & 6.1 \\
\hline 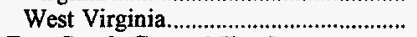 & 9,545 & 9,434 & 8,687 & 18,979 & 18,139 & 4.6 \\
\hline 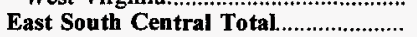 & 27,194 & 26,523 & 26,628 & $\mathbf{5 3 , 7 1 7}$ & $\mathbf{5 3 , 5 8 0}$ & .3 \\
\hline Alabama & 8,955 & 8,193 & 8,529 & 17,148 & 16,789 & 2.1 \\
\hline 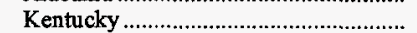 & 9,722 & 10,074 & 10,140 & 19,796 & 20,478 & -3.3 \\
\hline 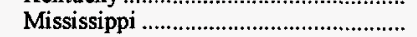 & 1,759 & 1,290 & 1,418 & 3,049 & 2,799 & 8.9 \\
\hline 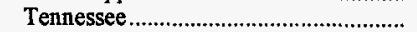 & 6,758 & 6,966 & 6,541 & 13,725 & 13,514 & 1.6 \\
\hline 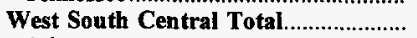 & 36,251 & 35,187 & 36,715 & 71,438 & 72,866 & -2.0 \\
\hline 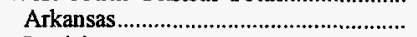 & 3,151 & 3,154 & 3,943 & 6,306 & 7,405 & -14.8 \\
\hline 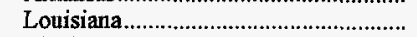 & 3,652 & 3,278 & 3,399 & 6,930 & 6,536 & 6.0 \\
\hline 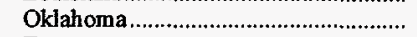 & 5,132 & 5,260 & 4,997 & 10,391 & 10,235 & 1.5 \\
\hline 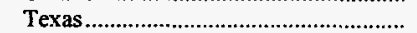 & 24,317 & 23,495 & 24,376 & 47,811 & 48,689 & -1.8 \\
\hline 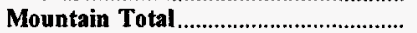 & 26,132 & 29,019 & 24,967 & 55,151 & 51,947 & 6.2 \\
\hline 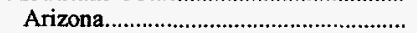 & 4,266 & 4,283 & 4,155 & 8,549 & 8,097 & 5.6 \\
\hline 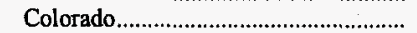 & 4,238 & 4,515 & 4,252 & 8,753 & 8,434 & 3.8 \\
\hline Idaho & 34 & 146 & 15 & 180 & 165 & 9.0 \\
\hline Montana & 2,339 & 2,696 & 1,761 & 5,035 & 4,017 & 25.3 \\
\hline 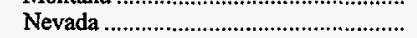 & 1,433 & 1,996 & 1,477 & 3,429 & 3,343 & 2.6 \\
\hline 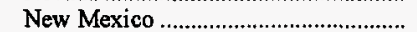 & 3,702 & 3,667 & 3,931 & 7,369 & 7,961 & -7.4 \\
\hline 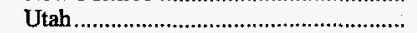 & 3,712 & 4,169 & 3,646 & 7,881 & 7,565 & 4.2 \\
\hline 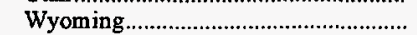 & 6,408 & 7,548 & 5,730 & 13,956 & 12,366 & 12.9 \\
\hline Pacific Total & 2,203 & 3,009 & 1,702 & 5,212 & 3,771 & 38.2 \\
\hline Alaska & 162 & 239 & 159 & 401 & 381 & 5.3 \\
\hline California & 710 & 632 & 544 & 1,342 & 1,073 & 25.1 \\
\hline 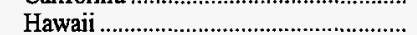 & 64 & 43 & 40 & 107 & 85 & 26.3 \\
\hline Oregon & 225 & 559 & $*$ & 784 & 80 & NM \\
\hline 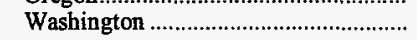 & 1,042 & 1,535 & 958 & 2,577 & 2,152 & 19.8 \\
\hline 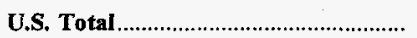 & 243,921 & 247,568 & 233,463 & 491,488 & 480,096 & 2.4 \\
\hline
\end{tabular}

* Rounded to zero.

NM Percent change calculation not meaningful as value is greater than 500

Notes: Consumption data exclude coal consumed by independent power producers to generate electricity and cogeneration plants not included in the other industrial, coke, and commercial sectors. See Table 37 Total may not equal sum of components because of independent rounding.

Sources: Energy Information Administration, Form EIA-759, "Monthly Power Plant Report;" Form EIA-3, "Quarterly Coal Consumption Report - Manufacturing Plants", Form EIA-867, "Annual Nonutility Power Producer Report," Form EIA-7A, "Coal Production Report," Form ElA-5, "Coke Plant Report Quarterly;" and Form EIA-6, "Coal Distribution Report." 
Table 39. Coal Consumption at Electric Utility Plants by Census Division and State (Thousand Short Tons)

\begin{tabular}{|c|c|c|c|c|c|c|}
\hline \multirow{2}{*}{$\begin{array}{l}\text { Census Division } \\
\text { and State }\end{array}$} & \multirow{2}{*}{$\begin{array}{c}\text { April - } \\
\text { June } \\
1998\end{array}$} & \multirow{2}{*}{$\begin{array}{c}\text { January - } \\
\text { March } \\
1998\end{array}$} & \multirow{2}{*}{$\begin{array}{l}\text { April - } \\
\text { June } \\
1997\end{array}$} & \multicolumn{3}{|c|}{ Year to Date } \\
\hline & & & & 1998 & 1997 & $\begin{array}{l}\text { Percent } \\
\text { Change }\end{array}$ \\
\hline 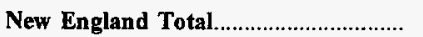 & $1, \mathbf{5 3 3}$ & 1,666 & 1,798 & 3,199 & 3,626 & -11.8 \\
\hline 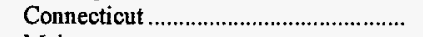 & 135 & 208 & 260 & 343 & 552 & -37.8 \\
\hline Maine & - & - & - & - & - & - \\
\hline 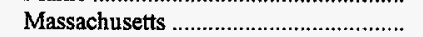 & 1,047 & 1,109 & 1,119 & 2,156 & 2,232 & -3.4 \\
\hline 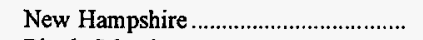 & 351 & 349 & 419 & 700 & 842 & -17.0 \\
\hline 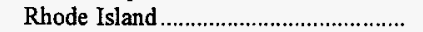 & - & - & - & - & - & - \\
\hline Vermont & - & - & - & - & - & - \\
\hline Middle Atlantic Total.............................. & 13,163 & 13,583 & 12,136 & 26,746 & 25,652 & 4.3 \\
\hline 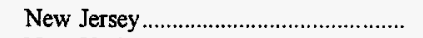 & 468 & 489 & 424 & 956 & 1,219 & -21.6 \\
\hline 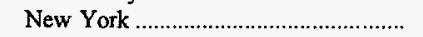 & 2,238 & 2,248 & 1,866 & 4,485 & 3,961 & 13.2 \\
\hline 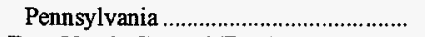 & 10,458 & 10,847 & 9,846 & 21,305 & 20,471 & 4.1 \\
\hline East North Central Total ...................... & 49,380 & $\mathbf{5 0 , 4 3 3}$ & 46,954 & 99,813 & 98,150 & 1.7 \\
\hline 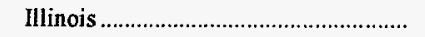 & 8,807 & 8,949 & 9,562 & 17,756 & 19,671 & -9.7 \\
\hline 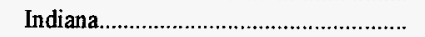 & 13,353 & 13,403 & 12,213 & 26,755 & 26,097 & 2.5 \\
\hline 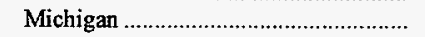 & 8,108 & 8,415 & 7,405 & 16,523 & 15,295 & 8.0 \\
\hline 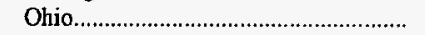 & 13,594 & 14,139 & 12,116 & 27,733 & 25,514 & 8.7 \\
\hline Wisconsin & 5,518 & 5,527 & 5,660 & 11,045 & 11,572 & -4.6 \\
\hline West North Central Total ........................ & 30,544 & 32,404 & 27,476 & 62,948 & 58,886 & 6.9 \\
\hline Iowa & 4,653 & 4,971 & 3,652 & 9,625 & 8,380 & 14.8 \\
\hline 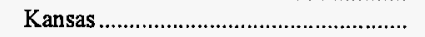 & 4,410 & 4,370 & 3,567 & 8,780 & 8,006 & 9.7 \\
\hline 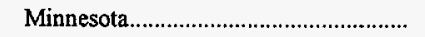 & 3,944 & 4,600 & 3,631 & 8,544 & 8,344 & 2.4 \\
\hline 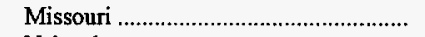 & 9,002 & 8,887 & 8,394 & 17,889 & 16,780 & 6.6 \\
\hline 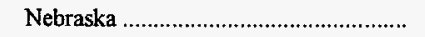 & 2,865 & 2,730 & 2,789 & 5,595 & 5,615 & -.3 \\
\hline 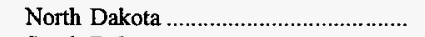 & 5,144 & 6,327 & 4,922 & 11,471 & 10,772 & 6.5 \\
\hline 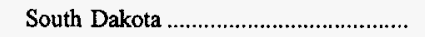 & 526 & 519 & 522 & 1,045 & 989 & 5.6 \\
\hline South Atlantic Total................................ & 39,419 & 35,978 & 36,290 & 75,397 & 72,371 & 4.2 \\
\hline 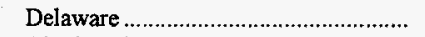 & 440 & 374 & 416 & 814 & 850 & -4.2 \\
\hline 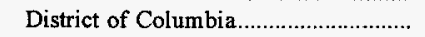 & - & - & - & - & - & - \\
\hline 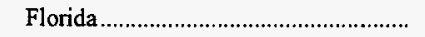 & 6,775 & 6,202 & 6,782 & 12,976 & 13,076 & -.8 \\
\hline 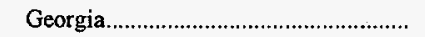 & 7,997 & 6,264 & 7,154 & 14,260 & 13,589 & 4.9 \\
\hline 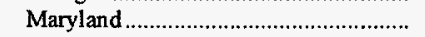 & 2,694 & 2,715 & 2,313 & 5,410 & 4,957 & 9.1 \\
\hline 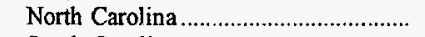 & 6,740 & 5,934 & 6,243 & 12,674 & 12,434 & 1.9 \\
\hline 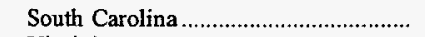 & 3,127 & 2,826 & 2,632 & 5,953 & 5,190 & 14.7 \\
\hline Virginia & 2,969 & 3,041 & 2,693 & 6,010 & 5,480 & 9.7 \\
\hline 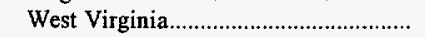 & 8,678 & 8,622 & 8,055 & 17,300 & 16,795 & 3.0 \\
\hline East South Central Total....................... & 24,048 & 23,230 & 23,273 & 47,278 & 46,697 & 1.2 \\
\hline Alabama & 7,650 & 6,869 & 7,105 & 14,520 & 13,923 & 4.3 \\
\hline 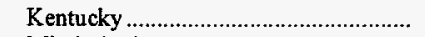 & 8,828 & 9,128 & 9,161 & 17,956 & 18,468 & -2.8 \\
\hline 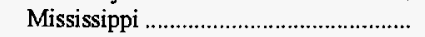 & 1,714 & 1,225 & 1,366 & 2,939 & 2,685 & 9.5 \\
\hline 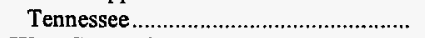 & 5,855 & 6,008 & 5,641 & 11,863 & 11,621 & 2.1 \\
\hline West South Central Total....................... & 34,702 & 33,646 & 35,265 & 68,349 & 69,885 & -2.2 \\
\hline 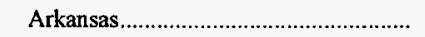 & 3,084 & 3,085 & 3,865 & 6,169 & 7,237 & -14.8 \\
\hline 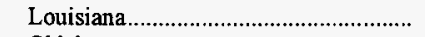 & 3,643 & 3,265 & 3,387 & 6,908 & 6,497 & 6.3 \\
\hline 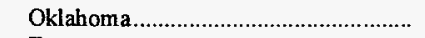 & 4,881 & 4,991 & 4,745 & 9,872 & 9,716 & 1.6 \\
\hline 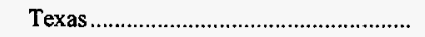 & 23,095 & 22,305 & 23,268 & 45,400 & 46,434 & -2.2 \\
\hline 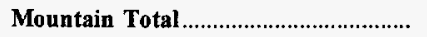 & 24,599 & 27,432 & 23,499 & 52,031 & 48,912 & 6.4 \\
\hline 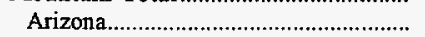 & 4,085 & 4,120 & 4,005 & 8,205 & 7,761 & 5.7 \\
\hline 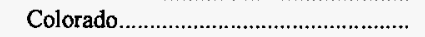 & 4,067 & 4,317 & 4,037 & 8,384 & 8,004 & 4.8 \\
\hline 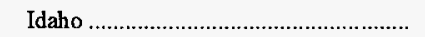 & - & - & - & - & - & - \\
\hline Montana & 2,312 & 2,641 & 1,722 & 4,953 & 3,905 & 26.8 \\
\hline Nevada & 1,380 & 1,951 & 1,435 & 3,331 & 3,258 & 2.2 \\
\hline 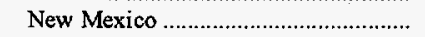 & 3,681 & 3,650 & 3,910 & 7,331 & 7,920 & -7.4 \\
\hline Utah & 3,164 & 3,738 & 3,165 & 6,902 & 6,726 & 2.6 \\
\hline 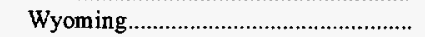 & 5,909 & 7,015 & 5,225 & 12,924 & 11,338 & 14.0 \\
\hline 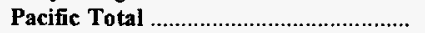 & 1,310 & 2,127 & 969 & $\mathbf{3 , 4 3 7}$ & 2,235 & 53.8 \\
\hline 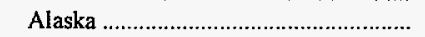 & 61 & 88 & 58 & 149 & 128 & 16.1 \\
\hline 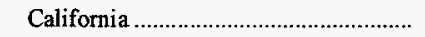 & - & - & - & - & - & - \\
\hline 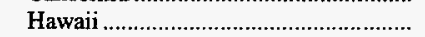 & - & - & - & - & - & - \\
\hline 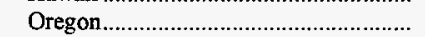 & 225 & 522 & - & 747 & 50 & NM \\
\hline Washington & 1,024 & 1,516 & 910 & 2,541 & 2,057 & 23.5 \\
\hline U.S. Total & 218,700 & 220,498 & 207,661 & 439,198 & 426,414 & 3.0 \\
\hline
\end{tabular}

NM Percent change calculation not meaningful as value is greater than 500 .

Note: Total may not equal sum of components because of independent rounding.

Source: Energy Information Administration, Form EIA-759, "Monthly Power Plant Report." 
Table 40. Change in Electric Utility Net Generation by State and Census Division, April-June 1998, 1997

(Million Kilowatthours)

\begin{tabular}{|c|c|c|c|c|c|c|c|c|c|c|c|c|}
\hline \multirow{2}{*}{$\begin{array}{l}\text { Census Division } \\
\text { and State }\end{array}$} & \multicolumn{2}{|c|}{ Total } & \multicolumn{2}{|c|}{ Coal } & \multicolumn{2}{|c|}{ Oil } & \multicolumn{2}{|c|}{ Gas } & \multicolumn{2}{|c|}{ Hydro } & \multicolumn{2}{|c|}{ Nuclear } \\
\hline & $\begin{array}{c}\text { Net } \\
\text { Change }\end{array}$ & $\begin{array}{l}\text { Percent } \\
\text { Change }\end{array}$ & $\begin{array}{c}\text { Net } \\
\text { Change }\end{array}$ & $\begin{array}{l}\text { Percent } \\
\text { Change }\end{array}$ & $\begin{array}{c}\text { Net } \\
\text { Change }\end{array}$ & $\begin{array}{c}\text { Percent } \\
\text { Change }\end{array}$ & $\begin{array}{c}\text { Net } \\
\text { Change }\end{array}$ & $\begin{array}{c}\text { Percent } \\
\text { Change }\end{array}$ & $\begin{array}{l}\text { Net } \\
\text { Change }\end{array}$ & $\begin{array}{c}\text { Percent } \\
\text { Change }\end{array}$ & $\begin{array}{l}\text { Net } \\
\text { Change }\end{array}$ & $\begin{array}{l}\text { Percent } \\
\text { Change }\end{array}$ \\
\hline New England . & 1,154 & 7 & 632 & 18 & -609 & -24 & $-1,096$ & -39 & 654 & 82 & 1,563 & 25 \\
\hline 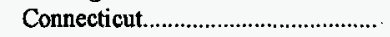 & $-2,271$ & -43 & 163 & 36 & -197 & -21 & -424 & -71 & 83 & 189 & $-1,922$ & -61 \\
\hline 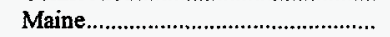 & 1,639 & 246 & - & - & -175 & -75 & - & - & 101 & 23 & 1,713 & - \\
\hline Massachusetts...................................... & 90 & 2 & 303 & 13 & -39 & -4 & $-1,334$ & -62 & 163 & -207 & 996 & 317 \\
\hline New Hampshire ................................ & 530 & 15 & 166 & 23 & -193 & -71 & -81 & -100 & 292 & 136 & 346 & 16 \\
\hline Rhode Island ............. & 741 & $\mathrm{NM}$ & * & - & -3 & -100 & 743 & NM & * & - & - & - \\
\hline 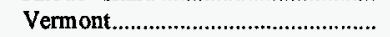 & 425 & 46 & - & - & -2 & -79 & $*$ & - & 14 & 7 & 430 & 61 \\
\hline Middle Atlantic ..................................... & 2,336 & 3 & 1,009 & 4 & -60 & -4 & $-3,115$ & -43 & 1,128 & 20 & 3,368 & 12 \\
\hline 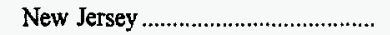 & $-2,881$ & -36 & -22 & -2 & 17 & 40 & -32 & -5 & 7 & -20 & $-2,851$ & -45 \\
\hline 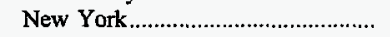 & 2,758 & 13 & -177 & -4 & -296 & -22 & $-2,710$ & -45 & 836 & 15 & 5,098 & 118 \\
\hline 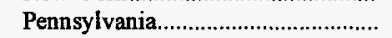 & 2,458 & 6 & 1,208 & 5 & 218 & 76 & -373 & -75 & 285 & 146 & 1,121 & 7 \\
\hline 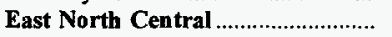 & -526 & * & $\mathbf{3 , 3 8 4}$ & 4 & 55 & 14 & 140 & 14 & -56 & -5 & $-4,071$ & -12 \\
\hline 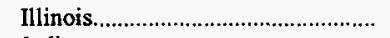 & $-1,008$ & -3 & 1,952 & 13 & 35 & 26 & 145 & 30 & -11 & -90 & $-3,136$ & -16 \\
\hline Indiana & 1,098 & 5 & 1,104 & 5 & 2 & 5 & 20 & 18 & -28 & -22 & - & - \\
\hline 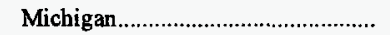 & -861 & -4 & $-1,058$ & -7 & 24 & 19 & -48 & -23 & -18 & -7 & 240 & 4 \\
\hline Ohio & 60 & * & 1,677 & 6 & 3 & 5 & 2 & 4 & 17 & 25 & $-1,640$ & -40 \\
\hline 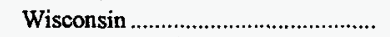 & 185 & 2 & -292 & -3 & -9 & -30 & 21 & 21 & -16 & -3 & 466 & 20 \\
\hline West North Central............................. & 3,320 & 6 & 1,008 & 2 & $-\mathbf{3 1}$ & -12 & -34 & -4 & 1,584 & 61 & 805 & 8 \\
\hline Iowa & 59 & 1 & -245 & -4 & 2 & 18 & 3 & 5 & -15 & -7 & 313 & 41 \\
\hline 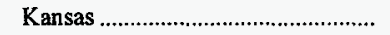 & 642 & 7 & 940 & 15 & 2 & 19 & 36 & 9 & - & - & -337 & -13 \\
\hline 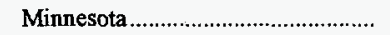 & -606 & -6 & -386 & -6 & 52 & 56 & -64 & -35 & 12 & 5 & -212 & -7 \\
\hline 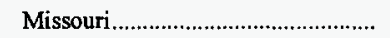 & 1,253 & 9 & 685 & 6 & -87 & -78 & -54 & -27 & -449 & -53 & 1,160 & 92 \\
\hline Nebraska & -385 & -6 & -398 & -11 & -2 & -22 & 37 & 97 & 100 & 30 & -119 & -5 \\
\hline 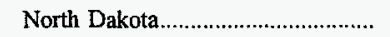 & 933 & 15 & 392 & 7 & 2 & 18 & $*$ & -103 & 538 & 162 & - & - \\
\hline 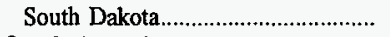 & 1,425 & 109 & 19 & 3 & * & -16 & 7 & 101 & 1,399 & 215 & - & - \\
\hline 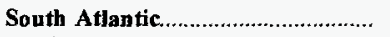 & 6,041 & 4 & 9,272 & 12 & 1,057 & 19 & $-1,089$ & -10 & 1,137 & 49 & $-4,337$ & -10 \\
\hline 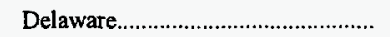 & 19 & 1 & -30 & -3 & 18 & 12 & 31 & 5 & - & - & - & - \\
\hline District of Columbia ............................ & 18 & NM & - & - & 18 & NM & - & - & - & - & - & - \\
\hline Florida & $-2,593$ & -7 & 588 & 4 & 704 & 14 & $-1,153$ & -11 & 2 & 3 & $-2,735$ & -33 \\
\hline 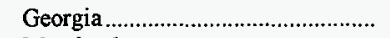 & $-1,278$ & -5 & -387 & -2 & 25 & 56 & 51 & 51 & 358 & 43 & $-1,325$ & -17 \\
\hline 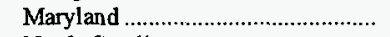 & 280 & 3 & 399 & 6 & 221 & 339 & -10 & -5 & 338 & 96 & -667 & -26 \\
\hline 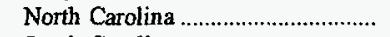 & 586 & 3 & 1,379 & 11 & -19 & -33 & 53 & 122 & 287 & 51 & $-1,114$ & -13 \\
\hline 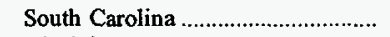 & 1,974 & 11 & 1,430 & 22 & 3 & 9 & -25 & -44 & 80 & 25 & 486 & 4 \\
\hline 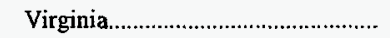 & 2,464 & 22 & 1,324 & 26 & 106 & $\mathrm{NM}$ & -26 & -9 & 42 & 66 & 1,017 & 18 \\
\hline 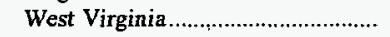 & 4,571 & 28 & 4,569 & 29 & -18 & -27 & -11 & -71 & 31 & 27 & - & - \\
\hline 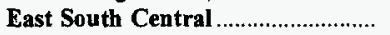 & 9,101 & 13 & 1,716 & 3 & 54 & 61 & -264 & -11 & 1,828 & 58 & 5,768 & 64 \\
\hline Alabama & 4,075 & 18 & 98 & 1 & 5 & 24 & 70 & 68 & 923 & 75 & 2,979 & 70 \\
\hline 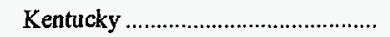 & 1,711 & 8 & 1,590 & 8 & -3 & -8 & 35 & 263 & 89 & 12 & - & - \\
\hline 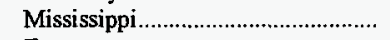 & 2,037 & 37 & 489 & 19 & 16 & 279 & -374 & -16 & - & - & 1,907 & 306 \\
\hline 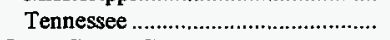 & 1,278 & 7 & -462 & -3 & 35 & 126 & 5 & 103 & 816 & 69 & 882 & 22 \\
\hline West South Central .............................. & 5,810 & 6 & 5,820 & 13 & -17 & -18 & 1,235 & 3 & $-1,238$ & -47 & 11 & * \\
\hline Arkansas & 1,577 & 16 & 1,342 & 31 & -3 & -17 & 402 & 46 & -139 & -17 & -26 & -1 \\
\hline 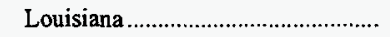 & $-1,843$ & -11 & -696 & -15 & 10 & 89 & $-1,458$ & -18 & - & - & 300 & 8 \\
\hline 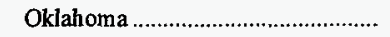 & 826 & 7 & 1,959 & 32 & -7 & -61 & -427 & -10 & -699 & -60 & - & - \\
\hline 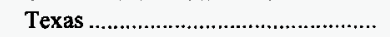 & 5,250 & 8 & 3,214 & 11 & -17 & -33 & 2,717 & 10 & -401 & -61 & -263 & -3 \\
\hline 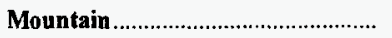 & 2,353 & 4 & $-1,227$ & -3 & -25 & -30 & 187 & 9 & 3,049 & 31 & 322 & 5 \\
\hline 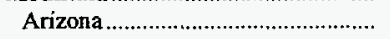 & 1,146 & 7 & -238 & -3 & -13 & -56 & 97 & 39 & 978 & 47 & 322 & 5 \\
\hline 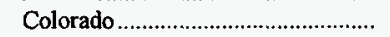 & 21 & $*$ & 91 & 1 & -1 & -30 & 24 & 34 & -93 & -15 & - & - \\
\hline 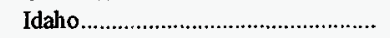 & 401 & 12 & - & - & * & -67 & - & - & 401 & 12 & - & - \\
\hline 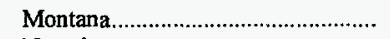 & 495 & 9 & -842 & -34 & -6 & -59 & $*$ & -7 & 1,343 & 49 & - & - \\
\hline 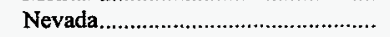 & 184 & 4 & -224 & -7 & -5 & -59 & 327 & 39 & 86 & 13 & - & - \\
\hline 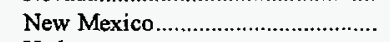 & 369 & 5 & 492 & 9 & -1 & -10 & -115 & -13 & -7 & -8 & - & - \\
\hline Utah & -593 & -9 & -512 & -8 & $*$ & -4 & -147 & -85 & 18 & 6 & - & - \\
\hline 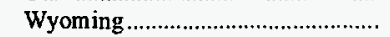 & 331 & 4 & 5 & * & 2 & 12 & 1 & 36 & 322 & 158 & - & - \\
\hline Pacific & 5,640 & 9 & 1,146 & 295 & 6 & 35 & -451 & -7 & 4,807 & 10 & -59 & -1 \\
\hline 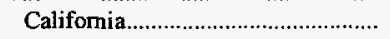 & $-1,801$ & -6 & - & - & 6 & 48 & -326 & -5 & $-1,950$ & -12 & 291 & 4 \\
\hline 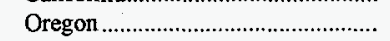 & 902 & 8 & -2 & 38 & * & -100 & -120 & -102 & 1,024 & 9 & - & - \\
\hline 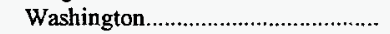 & 6,538 & 28 & 1,147 & 292 & $*$ & -19 & -4 & -96 & 5,732 & 26 & -350 & -102 \\
\hline Pacific Noncontiguous ....................... & 118 & 5 & * & 1 & 196 & 13 & 12 & 2 & -91 & -27 & - & - \\
\hline 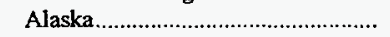 & -133 & -12 & $*$ & 1 & -56 & -75 & 12 & 2 & -90 & -28 & - & - \\
\hline 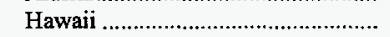 & 252 & 17 & - & - & 252 & 17 & - & - & $*$ & -8 & - & - \\
\hline 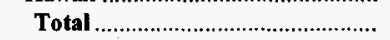 & 35,347 & 5 & 22,760 & 6 & 626 & 5 & $-4,476$ & -6 & 12,801 & 16 & 3,371 & 2 \\
\hline
\end{tabular}

* Number less than 0.5 rounded to zero.

NM Percent change calculation not meaningful as value is greater than 500 .

Source: Energy Information Administration, Form EIA-759, "Monthly Power Plant Report." 
Table 41. Coal Carbonized at Coke Plants by Census Division (Thousand Short Tons)

\begin{tabular}{|c|c|c|c|c|c|c|}
\hline \multirow[b]{2}{*}{ Census Division } & \multirow{2}{*}{$\begin{array}{c}\text { April - } \\
\text { June } \\
1998\end{array}$} & \multirow{2}{*}{$\begin{array}{c}\text { January - } \\
\text { March } \\
1998\end{array}$} & \multirow{2}{*}{$\begin{array}{c}\text { April - } \\
\text { June } \\
1997\end{array}$} & \multicolumn{3}{|c|}{ Year to Date } \\
\hline & & & & 1998 & 1997 & $\begin{array}{l}\text { Percent } \\
\text { Change }\end{array}$ \\
\hline 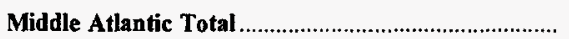 & 2,141 & 2,691 & 2,774 & 4,831 & 5,681 & -15.0 \\
\hline 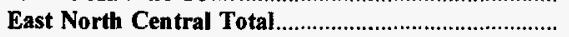 & 2,811 & 2,764 & 2,813 & 5,576 & 5,630 & -1.0 \\
\hline South Atlantic Total & $\mathrm{w}$ & $w$ & $\mathrm{w}$ & $w$ & $w$ & w \\
\hline East South Central Total. & 979 & 951 & 1,109 & 1,929 & 2,222 & -13.2 \\
\hline Mountain Total. & w & w & w & w & w & w \\
\hline U.S. Total & 6,929 & 7,279 & 7,410 & 14,208 & 15,000 & -5.3 \\
\hline
\end{tabular}

Withheld to avoid disclosure of individual company data.

Notes: Total may not equal sum of components because of independent rounding.

Source: Energy Information Administration, Form ELA-5, "Coke Plant Report - Quarterly." 
Table 42. Coal Consumption at Other Industrial Plants by Census Division and State (Thousand Short Tons)

\begin{tabular}{|c|c|c|c|c|c|c|}
\hline \multirow{2}{*}{$\begin{array}{l}\text { Census Division } \\
\text { and State }\end{array}$} & \multirow{2}{*}{$\begin{array}{l}\text { April - } \\
\text { June } \\
1998\end{array}$} & \multirow{2}{*}{$\begin{array}{c}\text { January - } \\
\text { March } \\
1998\end{array}$} & \multirow{2}{*}{$\begin{array}{l}\text { April - } \\
\text { June } \\
1997\end{array}$} & \multicolumn{3}{|c|}{ Year to Date } \\
\hline & & & & 1998 & 1997 & $\begin{array}{l}\text { Percent } \\
\text { Change }\end{array}$ \\
\hline New England Total.................................. & $\mathbf{w}$ & 79 & 53 & $\mathbf{w}$ & 120 & $\mathbf{w}$ \\
\hline Connecticut ........................................... & - & - & - & - & - & - \\
\hline Maine & $\mathbf{w}$ & $\mathbf{w}$ & $\mathbf{w}$ & $w$ & $w$ & $w$ \\
\hline 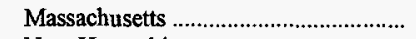 & $w$ & $\mathbf{w}$ & $\mathbf{w}$ & $\mathbf{w}$ & w & $\mathbf{w}$ \\
\hline 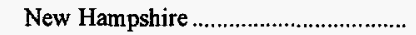 & - & - & - & - & - & - \\
\hline Rhode Island & - & - & - & - & - & - \\
\hline Vermont & 27 & 27 & - & 54 & - & - \\
\hline Middle Atlantic Total.............................. & 1,431 & $\mathbf{w}$ & $\mathbf{w}$ & 2,946 & $\mathbf{w}$ & $\mathbf{w}$ \\
\hline 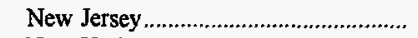 & $\mathbf{w}$ & $w$ & $w$ & $\mathbf{w}$ & $\mathbf{w}$ & $\mathbf{w}$ \\
\hline 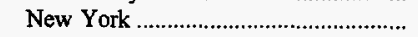 & $\mathbf{w}$ & 395 & 355 & $w$ & 718 & $\mathbf{w}$ \\
\hline 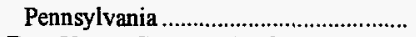 & 1,064 & 1,117 & 1,068 & 2,180 & 2,174 & 0.3 \\
\hline East North Central Total ....................... & 3,940 & 4,433 & 4,088 & $\mathbf{8 , 3 7 2}$ & 8,618 & -2.8 \\
\hline Illinois & 961 & 1,048 & 934 & 2,009 & 1,946 & 3.2 \\
\hline 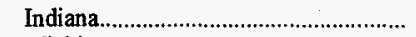 & 1,128 & 1,255 & 1,252 & 2,382 & 2,531 & -5.9 \\
\hline 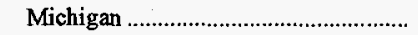 & 536 & 645 & 539 & 1,180 & 1,232 & -4.2 \\
\hline 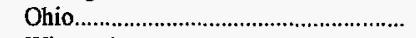 & 914 & 1,010 & 947 & 1,923 & 2,004 & -4.1 \\
\hline 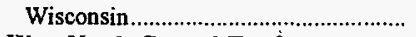 & 402 & 475 & 416 & 878 & 905 & -3.0 \\
\hline West North Central Total ........................ & 3,113 & 3,466 & 3,172 & 6,579 & 6,620 & -.6 \\
\hline 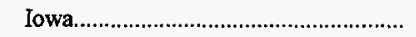 & 732 & 762 & 763 & 1,494 & 1,583 & -5.6 \\
\hline 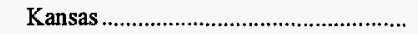 & 25 & 32 & 32 & 56 & 70 & -19.6 \\
\hline Minnesota & 348 & 481 & 329 & 829 & 780 & 6.2 \\
\hline 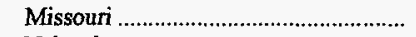 & 303 & 324 & 300 & 627 & 609 & 2.9 \\
\hline 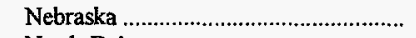 & $\mathbf{w}$ & $\mathbf{w}$ & $w$ & $\mathbf{w}$ & w & w \\
\hline 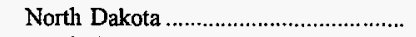 & $\mathbf{w}$ & $\mathbf{w}$ & $\mathbf{w}$ & $\mathbf{w}$ & $\mathbf{w}$ & $\mathbf{w}$ \\
\hline 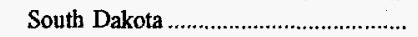 & 113 & 100 & 96 & 213 & 202 & 5.7 \\
\hline 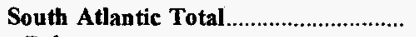 & $\mathbf{w}$ & $\mathbf{w}$ & $\mathbf{w}$ & $\mathbf{w}$ & $\mathbf{w}$ & $\mathbf{w}$ \\
\hline 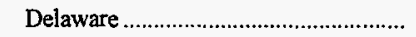 & w & $\mathbf{w}$ & w & $w$ & w & w \\
\hline 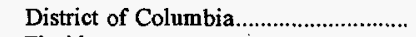 & - & - & - & - & - & - \\
\hline 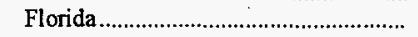 & 337 & 298 & 339 & 634 & 674 & -6.0 \\
\hline 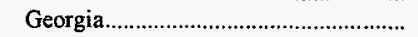 & 493 & 548 & 493 & 1,041 & 1,023 & 1.8 \\
\hline 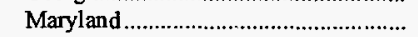 & 173 & 192 & 196 & 365 & 394 & -7.3 \\
\hline 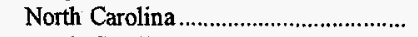 & 489 & 545 & 537 & 1,035 & 1,148 & -9.8 \\
\hline 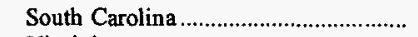 & 490 & 506 & 478 & 995 & 1,002 & -.7 \\
\hline Virginia & 564 & 659 & 644 & 1,223 & 1,324 & -7.6 \\
\hline 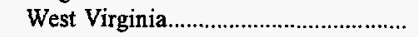 & 378 & 410 & 397 & 788 & 823 & -4.3 \\
\hline East South Central Total......................... & $\mathbf{w}$ & $\mathbf{w}$ & $\mathbf{w}$ & $\mathbf{w}$ & $\mathbf{w}$ & $\mathbf{w}$ \\
\hline Alabama & 661 & 697 & 629 & 1,358 & 1,272 & 6.7 \\
\hline 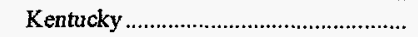 & 473 & 494 & 579 & 967 & 1,168 & -17.2 \\
\hline 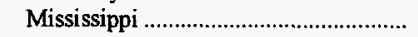 & $\mathbf{w}$ & w & w & w & $w$ & $w$ \\
\hline Tennessee & 877 & 919 & 874 & 1,796 & 1,827 & -1.7 \\
\hline West South Central Total. & 1,491 & 1,454 & 1,392 & 2,944 & 2,836 & 3.8 \\
\hline 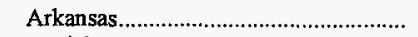 & 68 & 69 & 78 & 137 & 168 & -18.6 \\
\hline Louisiana & $\mathbf{w}$ & $\mathbf{w}$ & $w$ & $\mathbf{w}$ & w & $w$ \\
\hline 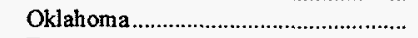 & $w$ & w & $\mathbf{w}$ & $w$ & $\mathbf{w}$ & $w$ \\
\hline 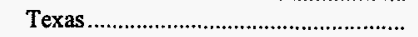 & 1,222 & 1,189 & 1,108 & 2,412 & 2,255 & 7.0 \\
\hline 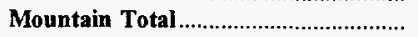 & 1,166 & 1,198 & 1,118 & 2,365 & 2,296 & 3.0 \\
\hline 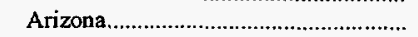 & 181 & 163 & 150 & 344 & 336 & 2.4 \\
\hline 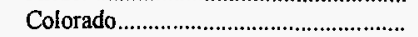 & 158 & 178 & 201 & 336 & 397 & -15.5 \\
\hline Idaho & 28 & w & $w$ & 165 & $\mathbf{w}$ & w \\
\hline Montana & $\mathbf{w}$ & $\mathbf{w}$ & $\mathbf{w}$ & $w$ & $w$ & w \\
\hline 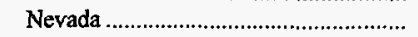 & $w$ & $\mathbf{w}$ & w & $\mathbf{w}$ & w & w \\
\hline 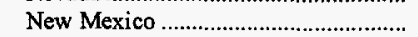 & w & $w$ & $w$ & $w$ & $w$ & $\mathbf{w}$ \\
\hline 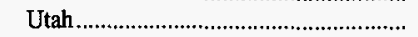 & 247 & 140 & 197 & 387 & 263 & 47.0 \\
\hline 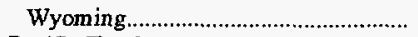 & 471 & 491 & 477 & 962 & 957 & .5 \\
\hline 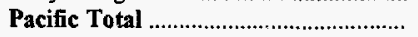 & 766 & 692 & 606 & 1,458 & 1,218 & 19.7 \\
\hline Alaska & w & $w$ & $\mathbf{w}$ & $w$ & $\mathrm{w}$ & w \\
\hline 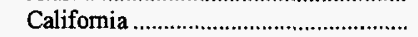 & 688 & 600 & 523 & 1,288 & 1,019 & 26.4 \\
\hline 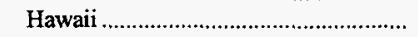 & w & $w$ & w & $\mathbf{w}$ & w & w \\
\hline Oregon & w & $w$ & $\mathbf{w}$ & $\mathbf{w}$ & $\mathbf{w}$ & $w$ \\
\hline 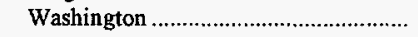 & $\mathbf{w}$ & 12 & 43 & $\mathrm{w}$ & 84 & w \\
\hline 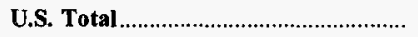 & 17,000 & 18,192 & 17,099 & 35,192 & 35,450 & -.7 \\
\hline
\end{tabular}

witheld to avoid disclosure of individual company data.

Note: Total may not equal sum of components because of independent rounding.

Sources: Energy Information Administration, Form EIA-3, "Quarterly Coal Consumption Report - Manufacturing Plants;" Form EIA-867, "Annual Nonutility Power Producer Report;" and Form ELA-7A, "Coal Production Report." 
Table 43. U.S. Coal Consumption at Manufacturing Plants by North American Industry Classification System (NAICS) Code

(Thousand Short Tons)

\begin{tabular}{|c|c|c|c|c|c|c|}
\hline \multirow[b]{2}{*}{ NAICS Code } & \multirow{2}{*}{$\begin{array}{c}\text { April - } \\
\text { June } \\
1998\end{array}$} & \multirow{2}{*}{$\begin{array}{c}\text { January - } \\
\text { March } \\
1998\end{array}$} & \multirow{2}{*}{$\begin{array}{c}\text { April - } \\
\text { June } \\
1997\end{array}$} & \multicolumn{3}{|c|}{ Year to Date } \\
\hline & & & & 1998 & 1997 & $\begin{array}{l}\text { Percent } \\
\text { Change }\end{array}$ \\
\hline 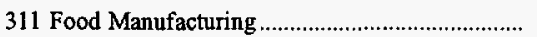 & 1,822 & 2,219 & 1,775 & 4,040 & 3,986 & 1.4 \\
\hline 312 Beverage and Tobacco Product Mfg...................... & 128 & 146 & 132 & 274 & 295 & -7.2 \\
\hline 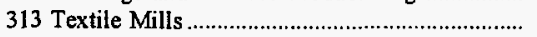 & 219 & 275 & 245 & 494 & 531 & -6.9 \\
\hline 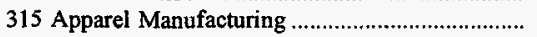 & $w$ & w & w & w & w & $\mathbf{w}$ \\
\hline 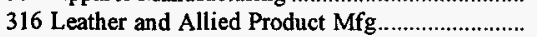 & - & $w$ & $\mathbf{w}$ & - & $\mathbf{w}$ & - \\
\hline 321 Wood Product Manufacturing................................. & $w$ & $w$ & $w$ & $w$ & $\mathbf{w}$ & $w$ \\
\hline 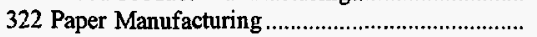 & 3,024 & 3,407 & 3,141 & 6,431 & 6,717 & -4.3 \\
\hline 323 Printing and Related Support............................... & - & - & - & - & - & - \\
\hline 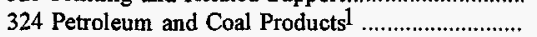 & w & 1,675 & 1,661 & $\mathbf{w}$ & 3,348 & $\mathbf{w}$ \\
\hline 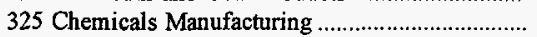 & 3,041 & 3,219 & 3,118 & 6,259 & 6,490 & -3.6 \\
\hline 326 Plastics and Rubber Products Mfg ........................ & 43 & 50 & 44 & 93 & 105 & -11.5 \\
\hline 327 Nonmetallic Mineral Products Mfg .................... & $\mathrm{w}$ & $w$ & $w$ & $\mathrm{w}$ & w & w \\
\hline 331 Primary Metal Manufacturing 2 & $\mathrm{w}$ & 2,249 & 2,114 & $\mathbf{w}$ & 4,114 & w \\
\hline 332 Fabricated Metal Product Mfg.............................. & 50 & 75 & 62 & 125 & 160 & -22.0 \\
\hline 333 Machinery Manufacturing & 48 & 119 & 67 & 166 & 221 & -24.8 \\
\hline 334 Computer and Electronic Product Mfg .............. & w & w & $\mathbf{w}$ & $\mathbf{w}$ & w & w \\
\hline 335 Elec. Equip., Appl., Components Mfg ............... & w & w & w & w & $\mathbf{w}$ & $\mathbf{w}$ \\
\hline 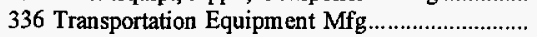 & 131 & 255 & 192 & 386 & 521 & -26.0 \\
\hline 337 Furniture and Related Product Mfg.................... & w & w & $\mathrm{w}$ & w & w & w \\
\hline 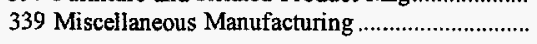 & w & $\mathbf{w}$ & w & $\mathbf{w}$ & w & w \\
\hline 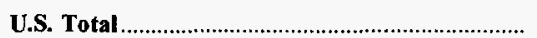 & 15,796 & 16,989 & 16,104 & 32,785 & 33,424 & -1.9 \\
\hline
\end{tabular}

1 Includes coal gasification projects.

2 Excludes coke plants.

withheld to avoid disclosure of individual company data.

Note: Total may not equal sum of components because of independent rounding.

Source: Energy Information Administration, Form EIA-3, “Quarterly Coal Consumption Report - Manufacturing Plants." 
Table 44. Coal Consumption by Residential and Commercial Sector by Census Division and State

(Thousand Short Tons)

\begin{tabular}{|c|c|c|c|c|c|c|}
\hline \multirow{2}{*}{$\begin{array}{l}\text { Census Division } \\
\text { and State }\end{array}$} & \multirow{2}{*}{$\begin{array}{l}\text { April - } \\
\text { June } \\
1998\end{array}$} & \multirow{2}{*}{$\begin{array}{c}\text { January - } \\
\text { March } \\
1998\end{array}$} & \multirow{2}{*}{$\begin{array}{l}\text { April - } \\
\text { June } \\
1997\end{array}$} & \multicolumn{3}{|c|}{ Year to Date } \\
\hline & & & & 1998 & 1997 & $\begin{array}{l}\text { Percent } \\
\text { Change }\end{array}$ \\
\hline New England Total. & 10 & 15 & 10 & 26 & 26 & 0.0 \\
\hline Connecticut & $\mathbf{w}$ & $\mathbf{w}$ & w & w & w & $\mathbf{w}$ \\
\hline Maine & w & $\mathbf{w}$ & $\mathbf{w}$ & $\mathbf{w}$ & w & $\mathbf{w}$ \\
\hline 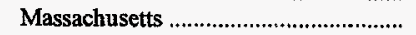 & $\mathbf{w}$ & w & $\mathbf{w}$ & $\mathrm{w}$ & $\mathbf{w}$ & w \\
\hline New Hampshire ........................................... & $w$ & w & w & w & w & w \\
\hline 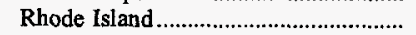 & $w$ & $\mathbf{w}$ & $\mathrm{w}$ & $\mathrm{w}$ & w & $\mathrm{w}$ \\
\hline 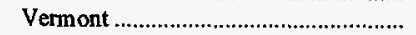 & w & w & $\mathrm{w}$ & w & $\mathbf{w}$ & $\mathrm{w}$ \\
\hline 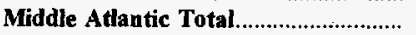 & 301 & 451 & 301 & 752 & 752 & .0 \\
\hline 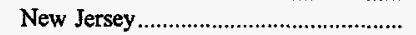 & $\mathbf{w}$ & w & w & w & $\mathbf{w}$ & $\mathrm{w}$ \\
\hline 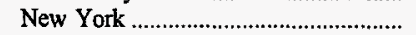 & $\mathbf{w}$ & w & w & w & w & $\mathrm{w}$ \\
\hline Pennsylvania & 249 & 373 & 249 & 622 & 622 & .0 \\
\hline East North Central Total ........................... & 274 & 411 & 274 & 685 & 685 & .0 \\
\hline 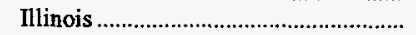 & $\mathbf{w}$ & w & w & $\mathbf{w}$ & $\mathbf{w}$ & w \\
\hline Indiana & 79 & 119 & 79 & 198 & 198 & .0 \\
\hline 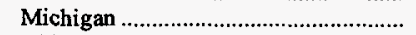 & $\mathbf{w}$ & w & w & $\mathbf{w}$ & $\mathbf{w}$ & $\mathrm{w}$ \\
\hline 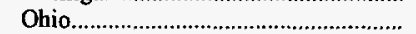 & 66 & 99 & 66 & 164 & 164 & .0 \\
\hline Wisconsin & $\mathbf{w}$ & $\mathbf{w}$ & $\mathrm{w}$ & $\mathbf{w}$ & $\mathbf{w}$ & $\mathrm{w}$ \\
\hline West North Central Total ........................ & $\mathbf{w}$ & $\mathbf{w}$ & w & $\mathbf{w}$ & $\mathbf{w}$ & w \\
\hline 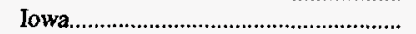 & 75 & 112 & 75 & 187 & 187 & .0 \\
\hline 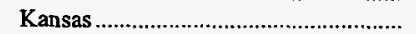 & $*$ & 1 & • & 1 & 1 & .0 \\
\hline 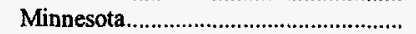 & 21 & 32 & 21 & 53 & 53 & .0 \\
\hline 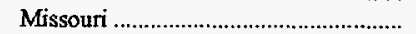 & $\mathbf{w}$ & $\mathbf{w}$ & w & $\mathrm{w}$ & $\mathbf{w}$ & $\mathrm{w}$ \\
\hline Nebraska ..................................................... & $\mathbf{w}$ & w & $\mathbf{w}$ & $\mathrm{w}$ & $\mathbf{w}$ & $\mathrm{w}$ \\
\hline 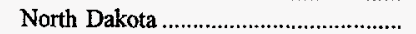 & $\mathbf{w}$ & $\mathbf{w}$ & $\mathbf{w}$ & $\mathbf{w}$ & $\mathbf{w}$ & $\mathbf{w}$ \\
\hline 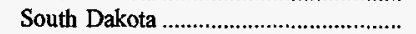 & $\mathrm{w}$ & w & w & w & $\mathbf{w}$ & $\mathrm{w}$ \\
\hline South Atlantic Total................................... & 120 & 180 & 120 & 299 & 299 & .0 \\
\hline Delaware & $\mathbf{w}$ & $\mathbf{w}$ & w & w & $\mathbf{w}$ & w \\
\hline District of Columbia................................. & 8 & 12 & 8 & 20 & 20 & .0 \\
\hline Florida & - & - & - & - & - & - \\
\hline 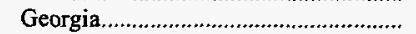 & 3 & 5 & 3 & 8 & 8 & .0 \\
\hline 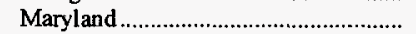 & $\mathbf{w}$ & $\mathbf{w}$ & $\mathbf{w}$ & w & $\mathbf{w}$ & w \\
\hline 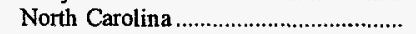 & 38 & 58 & 38 & 96 & 96 & .0 \\
\hline 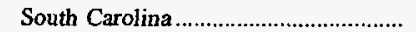 & * & $*$ & - & - & * & .0 \\
\hline 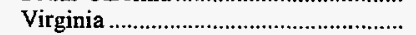 & w & $\mathbf{w}$ & $\mathbf{w}$ & w & $\mathbf{w}$ & $\mathrm{w}$ \\
\hline West Virginia & w & $\mathbf{w}$ & w & $\mathbf{w}$ & w & $\mathrm{w}$ \\
\hline East South Central Total......................... & 111 & 167 & 111 & 279 & 279 & .0 \\
\hline Alabama & 15 & 22 & 15 & 36 & 36 & .0 \\
\hline 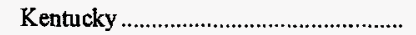 & w & $\mathbf{w}$ & w & w & w & $\mathrm{w}$ \\
\hline Mississippi & w & w & $\mathbf{w}$ & $\mathrm{w}$ & w & $\mathrm{w}$ \\
\hline 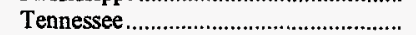 & w & $\mathbf{w}$ & w & w & $w$ & $\mathrm{w}$ \\
\hline West South Central Total.......................... & 58 & 87 & 58 & 145 & 145 & .0 \\
\hline 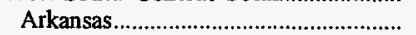 & $*$ & $*$ & * & * & $*$ & .0 \\
\hline 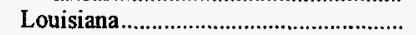 & w & w & $\mathbf{w}$ & $\mathbf{w}$ & w & $\mathrm{w}$ \\
\hline 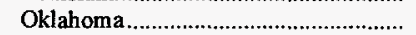 & w & w & w & $\mathbf{w}$ & w & $\mathrm{w}$ \\
\hline 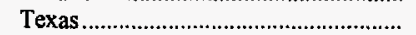 & $*$ & * & * & $*$ & * & .0 \\
\hline 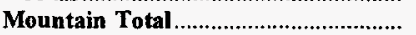 & $\mathbf{w}$ & $\mathbf{w}$ & $\mathbf{w}$ & w & $\mathbf{w}$ & $\mathbf{w}$ \\
\hline Arizona..................................................... & - & • & * & - & * & .0 \\
\hline Colorado & 13 & 19 & 13 & 32 & 32 & .0 \\
\hline 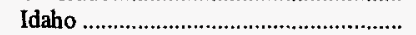 & 6 & 9 & 6 & 15 & 15 & .0 \\
\hline 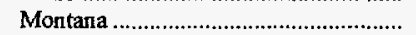 & w & w & w & $\mathbf{w}$ & $w$ & $\mathrm{w}$ \\
\hline 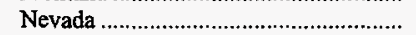 & w & w & $\mathrm{w}$ & $\mathbf{w}$ & w & $\mathrm{w}$ \\
\hline New Mexico ............................................... & w & w & w & $\mathbf{w}$ & w & $\mathbf{w}$ \\
\hline 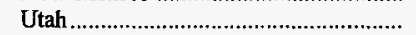 & w & $\mathbf{w}$ & $\mathbf{w}$ & $\mathbf{w}$ & $\mathbf{w}$ & w \\
\hline 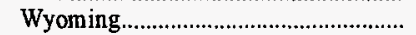 & 28 & 42 & 28 & 70 & 70 & .0 \\
\hline 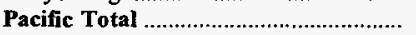 & 127 & 190 & 127 & 317 & 317 & .0 \\
\hline 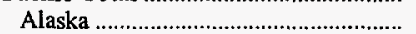 & 101 & 151 & 101 & 251 & 251 & .0 \\
\hline California & 22 & 33 & 22 & 54 & 54 & .0 \\
\hline 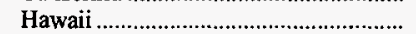 & $\mathbf{w}$ & w & w & w & $w$ & w \\
\hline Oregon & w & $\mathbf{w}$ & $\mathbf{w}$ & $\mathbf{w}$ & $\mathbf{w}$ & $\mathbf{w}$ \\
\hline 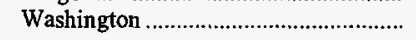 & 4 & 6 & 4 & 11 & 11 & .0 \\
\hline 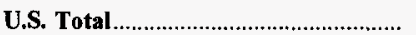 & 1,293 & 1,939 & 1,293 & 3,232 & $\mathbf{3 , 2 3 2}$ & .0 \\
\hline
\end{tabular}

* Rounded to zero.

Withheld to avoid disclosure of individual company data.

Note: Total may not equal sum of components because of independent rounding.

Source: Energy Information Administration, Form EIA-6, "Coal Distribution Report." 


\section{Stocks}


Table 45. U.S. Coal Stocks, 1992-1998

(Thousand Short Tons)

\begin{tabular}{|c|c|c|c|c|c|c|c|}
\hline \multirow{2}{*}{\multicolumn{2}{|c|}{ Last Day of Quarter }} & \multicolumn{4}{|c|}{ Coal Consumers 1} & \multirow{2}{*}{$\begin{array}{l}\text { Coal Producers } \\
\text { and Distributors }\end{array}$} & \multirow[b]{2}{*}{ Total } \\
\hline & & $\begin{array}{l}\text { Electric } \\
\text { Utilities }\end{array}$ & $\begin{array}{l}\text { Coke } \\
\text { Plants }\end{array}$ & $\begin{array}{c}\text { Other } \\
\text { Industrial } 2\end{array}$ & Total & & \\
\hline \multirow[t]{4}{*}{1992} & March 31 & 160,032 & 2,875 & 5,725 & 168,632 & 39,853 & 208,485 \\
\hline & June 30 & 164,176 & 2,776 & 6,317 & 173,270 & 40,513 & 213,783 \\
\hline & September $30 \ldots \ldots \ldots \ldots \ldots$ & 152,685 & 2,215 & 6,979 & 161,878 & 35,198 & 197,076 \\
\hline & 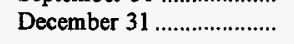 & 154,130 & 2,597 & 6,965 & 163,692 & 33,993 & 197,685 \\
\hline \multirow[t]{4}{*}{1993} & March 31 & 143,978 & 2,809 & 5,831 & 152,619 & 38,453 & 191,072 \\
\hline & June $30 \ldots \ldots \ldots \ldots \ldots \ldots \ldots$ & 145,753 & 3,020 & 6,070 & 154,842 & 34,827 & 189,669 \\
\hline & September 30 & 112,833 & 2,536 & 6,540 & 121,909 & 27,183 & 149,092 \\
\hline & December 31 ........................ & 111,341 & 2,401 & 6,716 & 120,458 & 25,284 & 145,742 \\
\hline \multirow[t]{4}{*}{1994} & March 31 & 105,186 & 2,232 & 4,859 & 112,278 & 34,139 & 146,417 \\
\hline & June $30 \ldots \ldots \ldots \ldots \ldots$ & 118,391 & 2,759 & 5,543 & 126,694 & 35,758 & 162,451 \\
\hline & September $30 \ldots \ldots \ldots$ & 112,314 & 2,706 & 6,206 & 121,225 & 32,955 & 154,180 \\
\hline & December 31 & 126,897 & 2,657 & 6,585 & 136,139 & 33,219 & 169,358 \\
\hline \multirow[t]{4}{*}{1995} & March 31 & 135,778 & 2,719 & 5,507 & 144,004 & 42,460 & 186,463 \\
\hline & June 30 & 143,385 & 2,624 & 5,649 & 151,657 & 42,104 & 193,761 \\
\hline & September 30 & 123,227 & 2,476 & 6,036 & 131,739 & 36,193 & 167,932 \\
\hline & December 31 ....................... & 126,304 & 2,632 & 5,702 & 134,639 & 34,444 & 169,083 \\
\hline \multirow[t]{4}{*}{1996} & March 31 & 117,738 & 2,583 & 4,431 & 124,752 & 36,851 & 161,603 \\
\hline & June 30 & 127,113 & 2,601 & 4,565 & 134,280 & 37,344 & 171,623 \\
\hline & September $30 \ldots \ldots \ldots \ldots$ & 119,473 & 2,814 & 5,301 & 127,588 & 33,780 & 161,368 \\
\hline & December 31 & 114,623 & 2,667 & 5,688 & 122,979 & 28,648 & 151,627 \\
\hline \multirow[t]{4}{*}{1997} & March 31 & 113,727 & 2,372 & 4,572 & 120,671 & 37,544 & 158,215 \\
\hline & June 30 & 120,787 & 2,050 & 4,751 & 127,588 & 42,529 & 170,117 \\
\hline & September 30 & 102,119 & 2,059 & 5,338 & 109,516 & 39,111 & 148,627 \\
\hline & December 31 & 98,826 & 1,978 & 5,597 & 106,401 & 33,973 & 140,374 \\
\hline \multirow[t]{2}{*}{1998} & March 31 & 107,540 & 1,986 & 4,588 & 114,114 & E 40,994 & E 155,108 \\
\hline & June 30 & 118,254 & 1,868 & 4,614 & 124,735 & E 38,331 & E 163,066 \\
\hline
\end{tabular}

1 Stock data for the Residential and Commercial sector are not included. See Technical Note 6 in Appendix C.

2 Manufacturing plants only.

E Estimated.

Notes: Total may not equal sum of components because of independent rounding.

Sources: Energy Information Administration (EIA) - Electric Utilities: Form EIA-759, "Monthly Power Plant Report" - Coke Plants: Form EIA-5,

"Coke Plant Report - Quarterly" - Other Industrial: Form EIA-3, "Quarterly Coal Consumption Report - Manufacturing Plants" and - Producer and Distributor: Form EIA-6, Schedule Q, "Quarterly Coal Report;" and, Form EIA-6, "Coal Distribution Report." 
Table 46. Consumer Coal Stocks by Census Division and State, June 30, 1998 (Thousand Short Tons)

\begin{tabular}{|c|c|c|c|c|}
\hline $\begin{array}{l}\text { Census Division } \\
\text { and State }\end{array}$ & $\begin{array}{l}\text { Electric } \\
\text { Utilities }\end{array}$ & $\begin{array}{l}\text { Coke } \\
\text { Plants }\end{array}$ & $\begin{array}{c}\text { Other } \\
\text { Industriall }\end{array}$ & Total \\
\hline 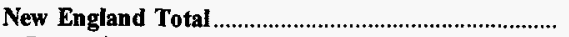 & 1,114 & - & 51 & 1,166 \\
\hline 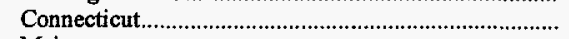 & 175 & - & $\mathbf{w}$ & w \\
\hline Maine & - & - & $\mathbf{w}$ & w \\
\hline 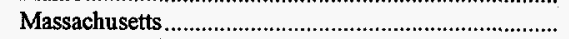 & 695 & - & w & w \\
\hline 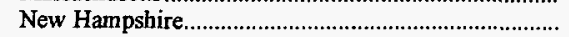 & 244 & - & $\mathbf{w}$ & $\mathbf{w}$ \\
\hline Rhode Island & - & - & $w$ & w \\
\hline 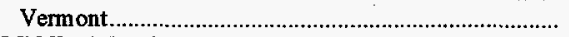 & - & - & $\mathbf{w}$ & w \\
\hline 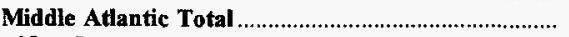 & 9,584 & 559 & $\mathbf{w}$ & $\mathbf{w}$ \\
\hline 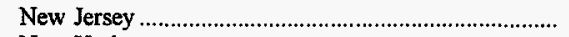 & 691 & - & $\mathbf{w}$ & w \\
\hline New York & 813 & $\mathbf{w}$ & 146 & w \\
\hline 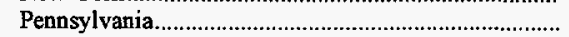 & 8,080 & w & $\mathrm{w}$ & 8,707 \\
\hline 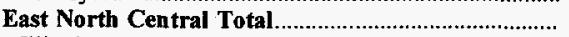 & 32,405 & 899 & 1,265 & 34,569 \\
\hline Illinois. & 6,634 & w & 235 & w \\
\hline Indiana & 7,670 & $\mathbf{w}$ & w & 8,471 \\
\hline Michigan & 8,662 & $\mathbf{w}$ & 403 & w \\
\hline Ohio & 5,231 & $\mathbf{w}$ & $w$ & 5,477 \\
\hline Wisconsin & 4,209 & - & 161 & 4,370 \\
\hline 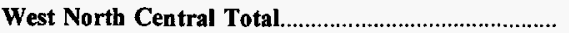 & 15,743 & - & 847 & 16,590 \\
\hline Iowa & 2,606 & - & 447 & 3,053 \\
\hline 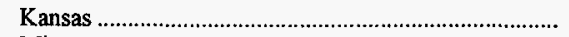 & 2,878 & - & 16 & 2,893 \\
\hline Minnesota & 1,881 & - & 168 & 2,048 \\
\hline 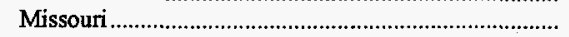 & 4,662 & - & 133 & 4,796 \\
\hline 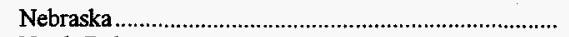 & 1,831 & - & $\mathbf{w}$ & w \\
\hline 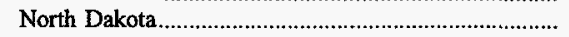 & 1,697 & - & $\mathbf{w}$ & w \\
\hline 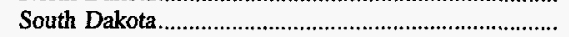 & 187 & - & $w$ & $w$ \\
\hline 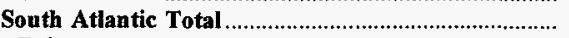 & 21,123 & $\mathbf{w}$ & $\mathbf{w}$ & w \\
\hline Delaware & 237 & - & $\mathbf{w}$ & w \\
\hline District of Columbia & - & - & - & - \\
\hline Florida & 4,471 & - & 122 & 4,594 \\
\hline Georgia & 3,041 & - & 142 & 3,183 \\
\hline Maryland & 1,273 & $\mathbf{w}$ & 15 & w \\
\hline 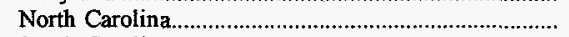 & 3,391 & - & 138 & 3,529 \\
\hline 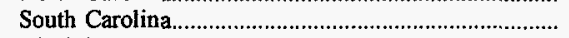 & 2,448 & - & 174 & 2,622 \\
\hline Virginia & 1,141 & $\mathbf{w}$ & 124 & w \\
\hline 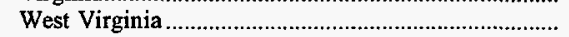 & 5,120 & w & 114 & $\mathbf{w}$ \\
\hline 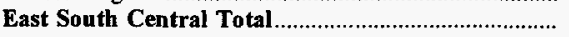 & 11,804 & 289 & $\mathbf{w}$ & $\mathbf{w}$ \\
\hline Alabama & 3,722 & $\mathbf{w}$ & $w$ & 4,099 \\
\hline 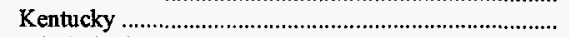 & 5,318 & $\mathbf{w}$ & 65 & $w$ \\
\hline 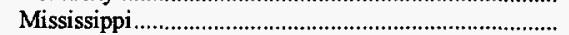 & 734 & - & $\mathbf{w}$ & $\mathbf{w}$ \\
\hline Tennessee & 2,031 & - & 222 & 2,253 \\
\hline 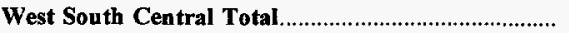 & 13,173 & - & 331 & 13,504 \\
\hline Arkansas & 1,375 & - & 26 & 1,401 \\
\hline 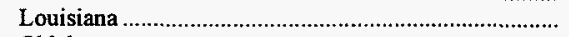 & 1,238 & - & 17 & 1,255 \\
\hline Oklahoma & 2,792 & - & 113 & 2,905 \\
\hline 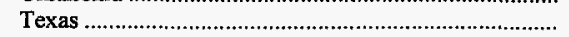 & 7,769 & - & 174 & 7,944 \\
\hline Mountain Total & 11,867 & $\mathbf{w}$ & 283 & $\mathbf{w}$ \\
\hline Arizona & 2,275 & - & 52 & 2,327 \\
\hline Colorado & 2,965 & - & 20 & 2,985 \\
\hline Idaho & - & - & 117 & 117 \\
\hline Montana & 418 & - & w & $\mathbf{w}$ \\
\hline Nevada & 839 & - & $\mathbf{w}$ & w \\
\hline New Mexico & 811 & - & $\mathbf{w}$ & $\mathbf{w}$ \\
\hline Utah & 2,981 & $\mathbf{w}$ & 5 & $\mathbf{w}$ \\
\hline Wyoming & 1,578 & - & 68 & 1,646 \\
\hline 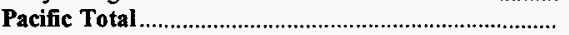 & 1,441 & - & 255 & 1,696 \\
\hline Alaska & * & - & - & * \\
\hline 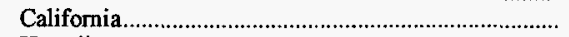 & - & - & 200 & 200 \\
\hline Hawaii & - & - & $\mathbf{w}$ & $\mathbf{w}$ \\
\hline Oregon & 283 & - & $\mathbf{w}$ & $\mathbf{w}$ \\
\hline Washington & 1,157 & - & 13 & 1,170 \\
\hline U.S. Total .............. & 118,254 & 1,868 & 4,614 & 124,735 \\
\hline
\end{tabular}

1 Manufacturing plants only.

* Rounded to zero.

withheld to avoid disclosure of individual company data.

Notes: Total may not equal sum of components because of independent rounding. Stock data for the Residential and Commercial sector are not available. See Technical Note 6 in Appendix C.

Sources: Energy Information Administration - Electric Utilities: Form EIA-759, "Monthly Power Plant Report" - Coke Plants: Form EIA-5, “Coke Plant Report - Quarterly" and • Other Industrial: Form EIA-3, "Quarterly Coal Consumption Report - Manufacturing Plants." 
Table 47. Coal Stocks at Electric Utility Plants by Census Division and State (Thousand Short Tons)

\begin{tabular}{|c|c|c|c|c|}
\hline $\begin{array}{c}\text { Census Division } \\
\text { and State }\end{array}$ & June 30, 1998 & March 31, 1998 & June 30, 1997 & $\begin{array}{c}\text { Percent Difference } \\
\text { June 30: } \\
1998 \text { versus } 1997\end{array}$ \\
\hline 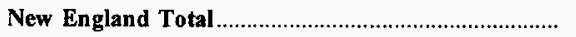 & 1,114 & 982 & 1,170 & -4.8 \\
\hline 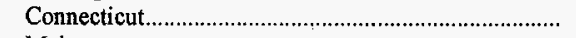 & 175 & 135 & 170 & 3.2 \\
\hline Maine & - & - & - & - \\
\hline Massachusetts . & 695 & 567 & 699 & -.5 \\
\hline 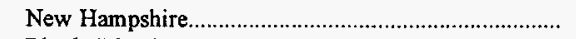 & 244 & 280 & 302 & -19.2 \\
\hline 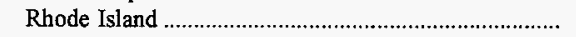 & - & - & - & - \\
\hline Vermont & - & - & - & - \\
\hline 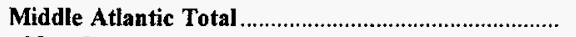 & 9,584 & 9,196 & 10,354 & -7.4 \\
\hline New Jersey & 691 & 588 & 919 & -24.8 \\
\hline New York & 813 & 719 & 765 & 6.3 \\
\hline Pennsylvania & 8,080 & 7,890 & 8,669 & -6.8 \\
\hline 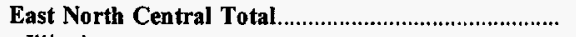 & 32,405 & 29,077 & 30,464 & 6.4 \\
\hline 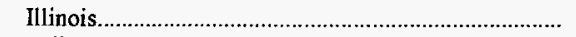 & 6,634 & 5,819 & 6,043 & 9.8 \\
\hline Indiana & 7,670 & 6,814 & 7,147 & 7.3 \\
\hline Michigan & 8,662 & 7,243 & 6,898 & 25.6 \\
\hline Ohio & 5,231 & 5,598 & 6,119 & -14.5 \\
\hline Wisconsin & 4,209 & 3,603 & 4,258 & -1.1 \\
\hline West North Central Total.............................................. & 15,743 & 14,419 & 16,230 & -3.0 \\
\hline Iowa & 2,606 & 1,941 & 3,895 & -33.1 \\
\hline Kansas & 2,878 & 2,660 & 2,973 & -3.2 \\
\hline 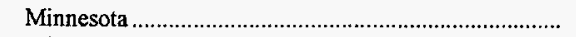 & 1,881 & 1,921 & 1,420 & 32.4 \\
\hline Missouri & 4,662 . & 4,133 & 4,317 & 8.0 \\
\hline 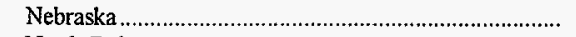 & 1,831 & 1,794 & 1,490 & 22.9 \\
\hline 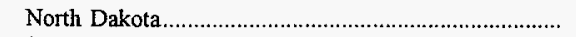 & 1,697 & 1,769 & 1,956 & -13.2 \\
\hline 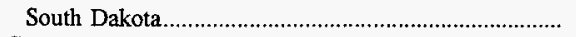 & 187 & 200 & 178 & 5.3 \\
\hline South Atlantic Total & 21,123 & 20,142 & 21,703 & -2.7 \\
\hline Delaware & 237 & 346 & 334 & -29.1 \\
\hline 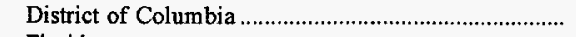 & - & - & - & - \\
\hline Florida & 4,471 & 4,004 & 3,579 & 24.9 \\
\hline Georgia & 3,041 & 3,638 & 4,587 & -33.7 \\
\hline 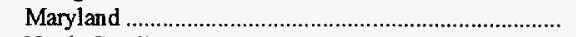 & 1,273 & 1,202 & 1,380 & -7.7 \\
\hline North Carolina & 3,391 & 3,314 & 3,388 & .1 \\
\hline 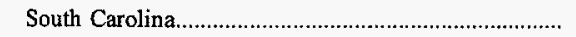 & 2,448 & 2,242 & 2,612 & -6.2 \\
\hline Virginia & 1,141 & 1,067 & 1,029 & 10.8 \\
\hline West Virginia & 5,120 & 4,329 & 4,795 & 6.8 \\
\hline 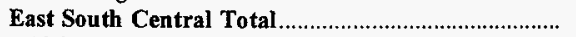 & 11,804 & 11,373 & 11,092 & 6.4 \\
\hline Alabama & 3,722 & 3,450 & 4,215 & -11.7 \\
\hline 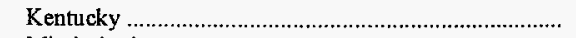 & 5,318 & 4,895 & 4,551 & 16.9 \\
\hline Mississippi & 734 & 835 & 789 & -6.9 \\
\hline Tennessee & 2,031 & 2,193 & 1,537 & 32.2 \\
\hline 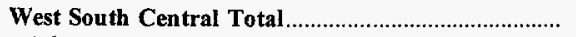 & 13,173 & 12,215 & 16,529 & -20.3 \\
\hline Arkansas & 1,375 & 919 & 1,583 & -13.2 \\
\hline 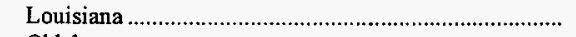 & 1,238 & 1,601 & 1,869 & -33.8 \\
\hline Oklahoma & 2,792 & 2,562 & 3,716 & -24.9 \\
\hline 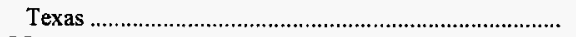 & 7,769 & 7,133 & 9,361 & -17.0 \\
\hline Mountain Total & 11,867 & 9,471 & 12,281 & -3.4 \\
\hline Arizona & 2,275 & 1,096 & 2,017 & 12.8 \\
\hline 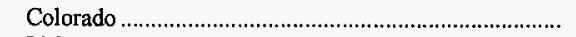 & 2,965 & 2,604 & 3,044 & -2.6 \\
\hline Idaho & - & - & - & - \\
\hline Montana & 418 & 432 & 501 & -16.7 \\
\hline Nevada & 839 & 1,043 & 1,275 & -34.2 \\
\hline New Mexico & 811 & 811 & 822 & -1.4 \\
\hline Utah & 2,981 & 2,353 & 2,683 & 11.1 \\
\hline Wyoming & 1,578 & 1,132 & 1,938 & -18.6 \\
\hline 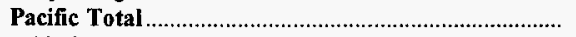 & 1,441 & 664 & 965 & 49.2 \\
\hline 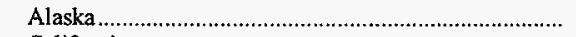 & $*$ & - & 1 & -87.5 \\
\hline 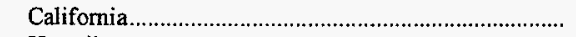 & - & - & - & - \\
\hline 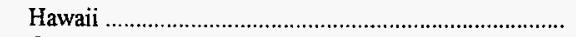 & - & - & - & - \\
\hline Oregon & 283 & 185 & 297 & -4.6 \\
\hline Washington & 1,157 & 480 & 668 & 73.4 \\
\hline U.S. Total & 118,254 & 107,540 & 120,787 & -2.1 \\
\hline
\end{tabular}

* Rounded to zero.

Note: Total may not equal sum of components because of independent rounding.

Source: Energy Information Administration, Form EIA-759, "Monthly Power Plant Report." 
Table 48. Coal Stocks at Coke Plants by Census Division

(Thousand Short Tons)

\begin{tabular}{|c|c|c|c|c|}
\hline Census Division & June 30, 1998 & March 31, 1998 & June 30, 1997 & $\begin{array}{c}\text { Percent Difference } \\
\text { June 30: } \\
1998 \text { versus } 1997\end{array}$ \\
\hline 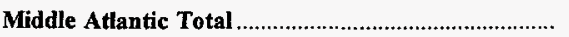 & 559 & 607 & 835 & -33.0 \\
\hline 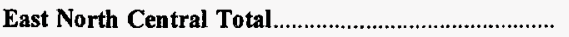 & 899 & 762 & 759 & 18.4 \\
\hline 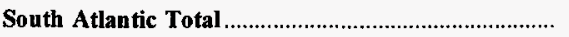 & $\mathbf{w}$ & $\mathbf{w}$ & w & w \\
\hline 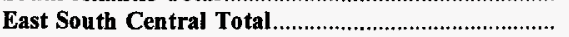 & 289 & 318 & 291 & -.7 \\
\hline 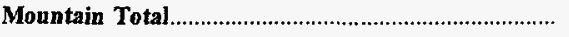 & w & $w$ & $\mathbf{w}$ & $w$ \\
\hline U.S. Total & 1,868 & 1,812 & 2,050 & -8.9 \\
\hline
\end{tabular}

Withheld to avoid disclosure of individual company data.

Notes: Total may not equal sum of components because of independent rounding.

Source: Energy Information Administration, Form EIA-5, "Coke Plant Report - Quarterly." 
Table 49. Coal Stocks at Other Industrial Plants by Census Division and State (Thousand Short Tons)

\begin{tabular}{|c|c|c|c|c|}
\hline $\begin{array}{c}\text { Census Division } \\
\text { and State }\end{array}$ & June 30, 1998 & March 31, 1998 & June 30, 1997 & $\begin{array}{c}\text { Percent Difference } \\
\text { June 30: } \\
1998 \text { versus } 1997\end{array}$ \\
\hline 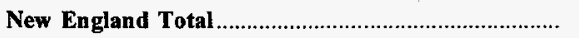 & $\mathbf{w}$ & $\mathbf{5 5}$ & $\mathbf{5 3}$ & $\mathbf{w}$ \\
\hline 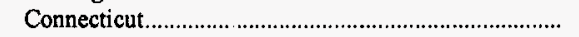 & - & - & - & - \\
\hline Maine & $\mathbf{w}$ & w & $\mathbf{w}$ & $\mathbf{w}$ \\
\hline 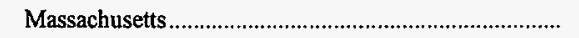 & $\mathbf{w}$ & $\mathbf{w}$ & $\mathbf{w}$ & $\mathbf{w}$ \\
\hline New Hampshire & - & - & - & - \\
\hline Rhode Island & - & - & - & - \\
\hline Verm ont & - & - & - & - \\
\hline 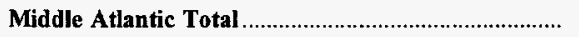 & 278 & $\mathbf{w}$ & $\mathbf{w}$ & $\mathbf{w}$ \\
\hline 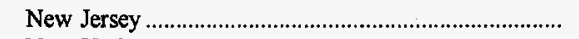 & $\mathbf{w}$ & $\mathbf{w}$ & w & $\mathbf{w}$ \\
\hline New York & $\mathbf{w}$ & 123 & 114 & $\mathbf{w}$ \\
\hline Pennsylvania & 132 & 170 & 197 & -32.9 \\
\hline 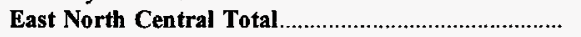 & 1,265 & 1,353 & 1,367 & -7.5 \\
\hline 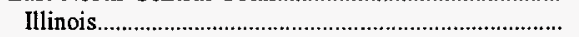 & 235 & 210 & 243 & -3.4 \\
\hline 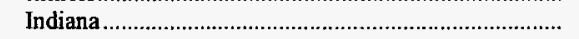 & 314 & 296 & 319 & -1.5 \\
\hline Michigan & 403 & 474 & 455 & -11.5 \\
\hline Ohio & 152 & 178 & 162 & -6.1 \\
\hline Wisconsin & 161 & 194 & 188 & -14.7 \\
\hline 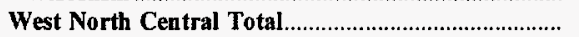 & 847 & 676 & 869 & -2.5 \\
\hline 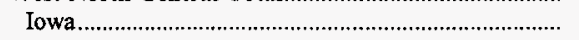 & 447 & 317 & 374 & 19.5 \\
\hline 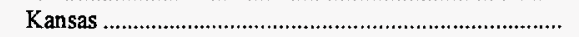 & 16 & 17 & 16 & -3.1 \\
\hline Minnesota & 168 & 119 & 182 & -7.7 \\
\hline 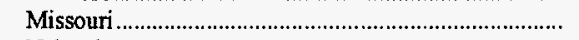 & 133 & 151 & 182 & -26.8 \\
\hline Nebraska & $\mathbf{w}$ & $\mathbf{w}$ & $w$ & w \\
\hline 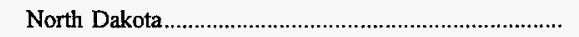 & $\mathbf{w}$ & w & $w$ & w \\
\hline 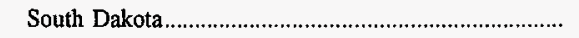 & 19 & 21 & 28 & -32.5 \\
\hline South Atlantic Total & $\mathbf{w}$ & $\mathbf{w}$ & $\mathbf{w}$ & $\mathbf{w}$ \\
\hline 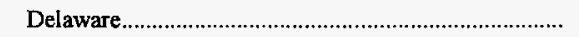 & $w$ & w & $\mathbf{w}$ & w \\
\hline District of Columbia & - & - & - & - \\
\hline Florida & 122 & 103 & 93 & 31.6 \\
\hline 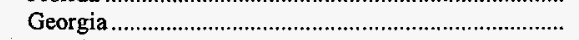 & 142 & 145 & 122 & 15.9 \\
\hline Maryland & 15 & 14 & 26 & -42.0 \\
\hline North Carolina. & 138 & 131 & 138 & * \\
\hline South Carolina & 174 & 181 & 212 & -17.7 \\
\hline 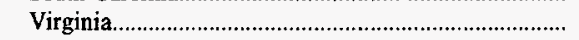 & 124 & 133 & 131 & -5.8 \\
\hline 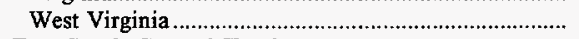 & 114 & 121 & 127 & -10.2 \\
\hline 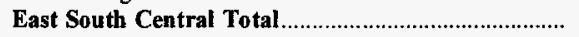 & $\mathbf{w}$ & $\mathbf{w}$ & $\mathbf{w}$ & $\mathbf{w}$ \\
\hline 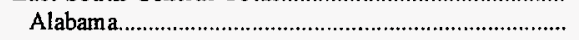 & 179 & 186 & 169 & 5.8 \\
\hline Kentucky & 65 & 89 & 75 & -14.1 \\
\hline Mississippi & $\mathbf{w}$ & $\mathbf{w}$ & $\mathbf{w}$ & w \\
\hline Tennessee & 222 & 205 & 231 & -4.0 \\
\hline 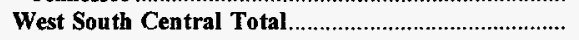 & 331 & 318 & 335 & -1.2 \\
\hline 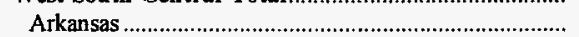 & 26 & 21 & 18 & 45.7 \\
\hline Louisiana & w & $w$ & $\mathbf{w}$ & $\mathbf{w}$ \\
\hline Oklahoma & w & $\mathbf{w}$ & $\mathbf{w}$ & $\mathbf{w}$ \\
\hline 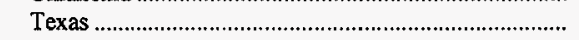 & 174 & 166 & 194 & -10.4 \\
\hline 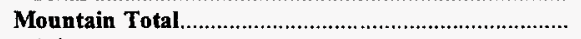 & 283 & 243 & 260 & 9.1 \\
\hline Arizona & 52 & 38 & 35 & 46.8 \\
\hline Colorado & 20 & 20 & 15 & 34.7 \\
\hline Idaho & 117 & w & $\mathbf{w}$ & w \\
\hline 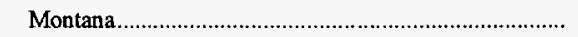 & $\mathbf{w}$ & $w$ & $\mathbf{w}$ & $\mathbf{w}$ \\
\hline 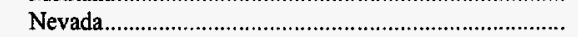 & $\mathbf{w}$ & w & $w$ & w \\
\hline New Mexico & $w$ & $\mathbf{w}$ & $\mathbf{w}$ & w \\
\hline Utah & 5 & 3 & 4 & 11.5 \\
\hline Wyoming & 68 & 85 & 81 & -15.1 \\
\hline Pacific Total & 255 & 326 & 211 & 20.8 \\
\hline 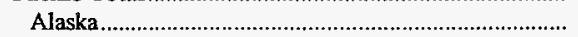 & w & w & $\mathbf{w}$ & w \\
\hline 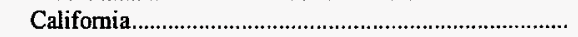 & 200 & 210 & 125 & 59.3 \\
\hline 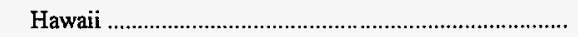 & $\mathbf{w}$ & $\mathbf{w}$ & w & w \\
\hline Oregon & $\mathbf{w}$ & $\mathbf{w}$ & $\mathbf{w}$ & $\mathbf{w}$ \\
\hline 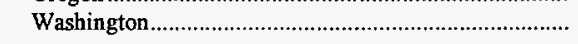 & $\mathbf{w}$ & 15 & 23 & $\mathbf{w}$ \\
\hline U.S. Total & 4,614 & 4,588 & 4,751 & -2.9 \\
\hline
\end{tabular}

* Rounded to zero.

With held to avoid disclosure of individual company data.

Notes: Total may not equal sum of components because of independent rounding. Other industrial plants include manufacturing plants only

Source: Energy Information Administration, Form EIA-3, "Quarterly Coal Consumption Report - Manufacturing Plants." 
Table 50. U.S. Coal Stocks at Manufacturing Plants by North American Industry Classification System (NAICS) Code

(Thousand Short Tons)

\begin{tabular}{|c|c|c|c|c|}
\hline NAICS Code & June 30, 1998 & March 31, 1998 & June 30, 1997 & $\begin{array}{c}\text { Percent Difference } \\
\text { June 30: } \\
1998 \text { versus } 1997\end{array}$ \\
\hline 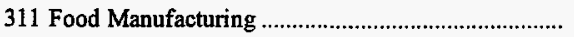 & 679 & 585 & 610 & 11.4 \\
\hline 312 Beverage and Tobacco Product Mfg ............................ & 25 & 27 & 24 & 5.4 \\
\hline 313 Textile Mills & 89 & 105 & 87 & 2.9 \\
\hline 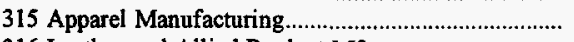 & w & $\mathbf{w}$ & $\mathbf{w}$ & $\mathbf{w}$ \\
\hline 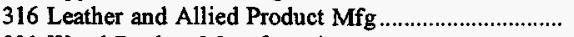 & - & $\mathbf{w}$ & $\mathbf{w}$ & $\mathbf{w}$ \\
\hline 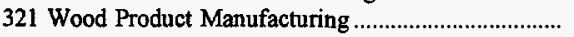 & $\mathbf{w}$ & w & $\mathbf{w}$ & $\mathbf{w}$ \\
\hline 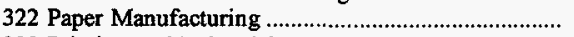 & 811 & 932 & 853 & -5.0 \\
\hline 323 Printing and Related Support & - & - & - & - \\
\hline 324 Petroleum and Coal Products 1 & $\mathbf{w}$ & 51 & 71 & $\mathbf{w}$ \\
\hline 325 Chemicals Manufacturing & 738 & 766 & 869 & -15.1 \\
\hline 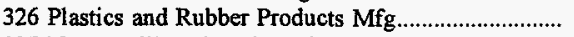 & 5 & 6 & 11 & -53.4 \\
\hline 327 Nonmetallic Mineral Products Mfg ............................ & $\mathbf{w}$ & $\mathbf{w}$ & w & $\mathbf{w}$ \\
\hline 331 Primary Metal Manufacturing 2 & $\mathbf{w}$ & 519 & 638 & $\mathbf{w}$ \\
\hline 332 Fabricated Metal Product Mfg & 31 & 42 & 31 & .9 \\
\hline 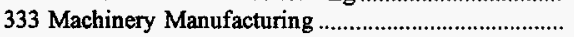 & 45 & 42 & 28 & 57.8 \\
\hline 334 Computer and Electronic Product $\mathrm{Mfg}$ & $\mathbf{w}$ & $\mathbf{w}$ & $\mathbf{w}$ & $\mathbf{w}$ \\
\hline 335 Elec. Equip., Appl., Components Mfg .................... & $\mathbf{w}$ & w & $\mathbf{w}$ & w \\
\hline 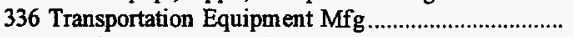 & 44 & 61 & 67 & -35.1 \\
\hline 337 Fumiture and Related Product Mfg ......................... & $\mathbf{w}$ & $\mathbf{w}$ & $\mathbf{w}$ & $\mathbf{w}$ \\
\hline 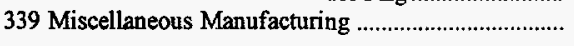 & $\mathbf{w}$ & $\mathbf{w}$ & $w$ & $\mathbf{w}$ \\
\hline 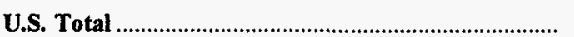 & 4,614 & 4,588 & 4,751 & -2.9 \\
\hline
\end{tabular}

1 Inciudes coal gasification projects.

2 Excludes coke plants.

withheld to avoid disclosure of individual company data.

Note: Total may not equal sum of components because of independent rounding

Source: Energy Information Administration, Form EIA-3, "Quarterly Coal Consumption Report - Manufacturing Plants."

Table 51. Coke and Breeze Stocks at Coke Plants by Census Division (Thousand Short Tons)

\begin{tabular}{|c|c|c|c|c|}
\hline Census Division & June 30, 1998 & March 31, 1998 & June 30, 1997 & $\begin{array}{c}\text { Percent Difference } \\
\text { June 30: } \\
1998 \text { versus } 1997\end{array}$ \\
\hline 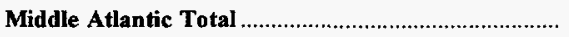 & 166 & 332 & 281 & -41.1 \\
\hline 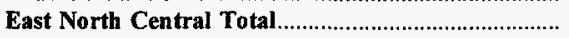 & 552 & 469 & 549 & .5 \\
\hline South Atlantic Total & $\mathbf{w}$ & $w$ & $\mathbf{w}$ & $\mathbf{w}$ \\
\hline 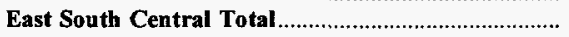 & 168 & 140 & 229 & -26.7 \\
\hline Mountain Total. & w & w & $\mathbf{w}$ & $w$ \\
\hline U.S. Total & 1,054 & 1,237 & 1,653 & -36.2 \\
\hline 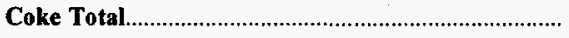 & 866 & 1,084 & 1,522 & -43.1 \\
\hline Breeze Total. & 189 & 153 & 131 & 44.2 \\
\hline
\end{tabular}

withheld to avoid disclosure of individual company data.

Note: Total may not equal sum of components because of independent rounding.

Source: Energy Information Administration, Form EIA-5, "Coke Plant Report - Quarterly." 


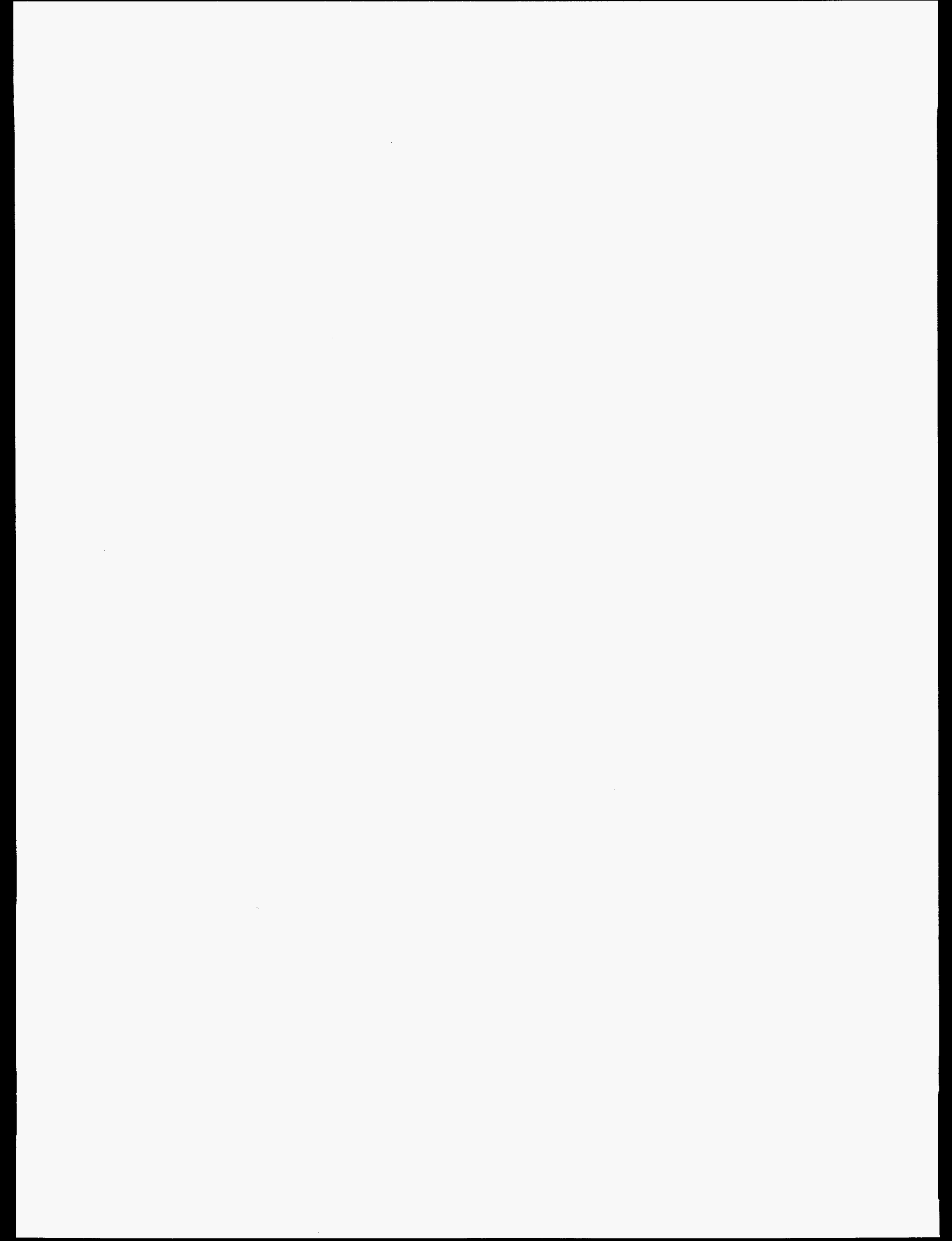


Appendix A 


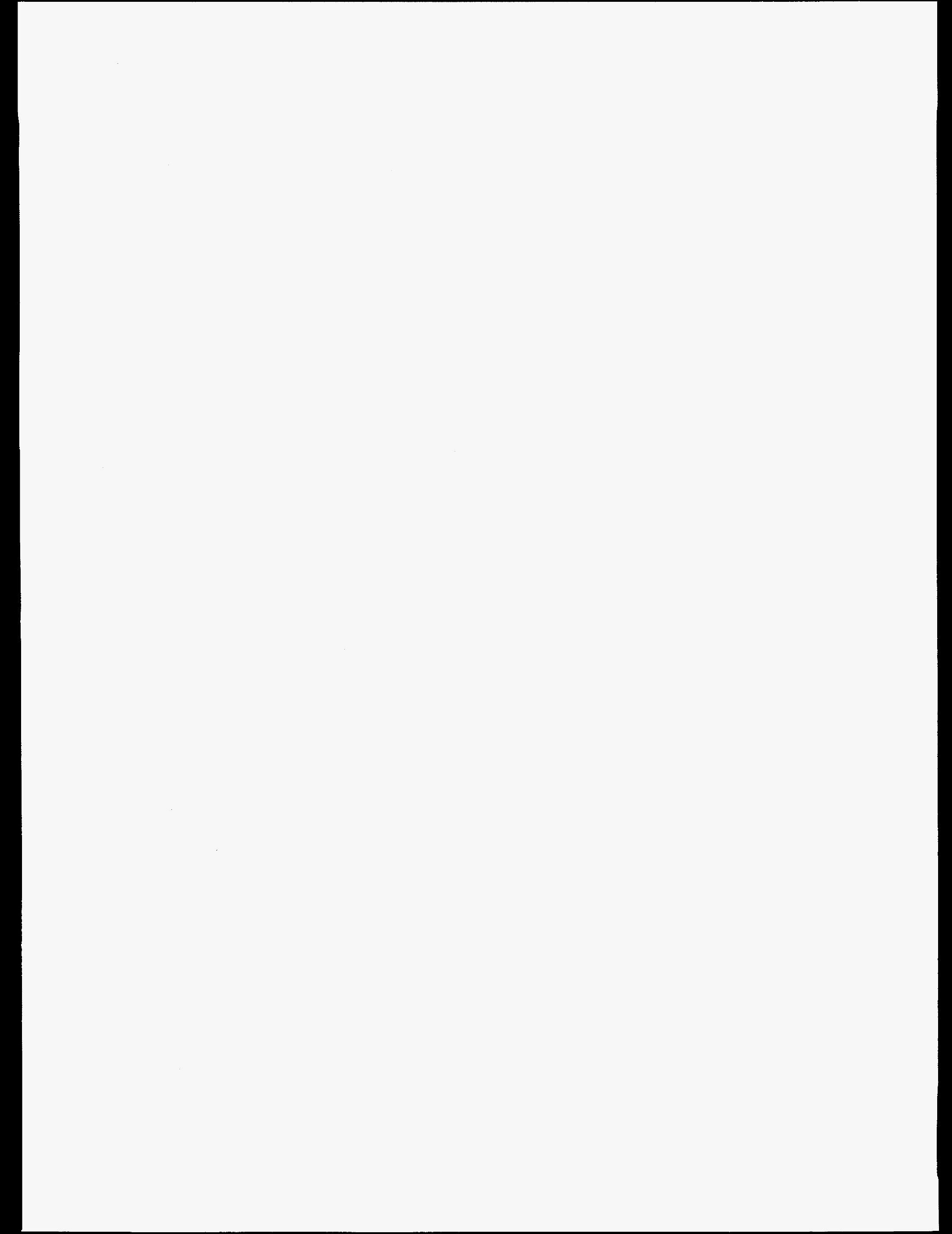


Table A1. Quantity and Average Price of U.S. Coal Imports, 1992-1998 (Thousand Short Tons and Dollars per Short Ton)

\begin{tabular}{|c|c|c|c|c|c|c|c|c|c|c|}
\hline \multirow[b]{2}{*}{ Year } & \multicolumn{2}{|c|}{ January - March } & \multicolumn{2}{|c|}{ April - June } & \multicolumn{2}{|c|}{ July - September } & \multicolumn{2}{|c|}{ October - December } & \multicolumn{2}{|c|}{ U.S. Total } \\
\hline & Quantity & $\begin{array}{l}\text { Average } \\
\text { Price }\end{array}$ & Quantity & $\begin{array}{c}\text { Average } \\
\text { Price }\end{array}$ & Quantity & $\begin{array}{c}\text { Average } \\
\text { Price }\end{array}$ & Quantity & $\begin{array}{c}\text { Average } \\
\text { Price }\end{array}$ & Quantity & $\begin{array}{c}\text { Average } \\
\text { Price }\end{array}$ \\
\hline $1992 \ldots \ldots \ldots \ldots \ldots$ & 679 & $\$ 33.63$ & 1,043 & $\$ 32.96$ & 882 & $\$ 34.43$ & 1,199 & $\$ 33.08$ & 3,803 & $\$ 33.46$ \\
\hline $1993 \ldots \ldots \ldots \ldots \ldots \ldots$ & 1,213 & 30.70 & 1,093 & 32.26 & 2,142 & 29.52 & 2,861 & 28.91 & 7,309 & 29.89 \\
\hline $1994 \ldots \ldots \ldots \ldots \ldots \ldots$ & 1,850 & 28.86 & 1,577 & 28.73 & 2,304 & 30.92 & 1,853 & 31.93 & 7,584 & 30.21 \\
\hline $1995 \ldots \ldots \ldots \ldots \ldots \ldots$ & 1,795 & 32.33 & 1,609 & 36.16 & 1,725 & 33.61 & 2,071 & 34.54 & 7,201 & 34.13 \\
\hline $1996 \ldots \ldots \ldots \ldots \ldots \ldots$ & 1,713 & 33.52 & 1,552 & 32.46 & 2,071 & 33.19 & 1,790 & 34.55 & 7,126 & 33.45 \\
\hline 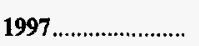 & 1,331 & 33.85 & 1,708 & 35.26 & 2,222 & 33.69 & 2,226 & 34.49 & 7,487 & $\mathbf{3 4 . 3 2}$ \\
\hline 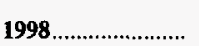 & 1,839 & 33.98 & 2,193 & 31.43 & NA & NA & NA & $\mathrm{NA}$ & 4,031 & 32.59 \\
\hline
\end{tabular}

NA Not available.

Notes: Average price is based on the customs import value. Total may not equal sum of components because of independent rounding. Coal imports include coal to Puerto Rico and the Virgin Islands.

Source: Bureau of the Census, U.S. Department of Commerce, 'Monthly Report IM 145." 
Table A2. Quantity and Average Price of U.S. Coal Imports by Origin, 1992-1998

(Thousand Short Tons and Dollars per Short Ton)

\begin{tabular}{|c|c|c|c|c|c|c|c|c|}
\hline Year and Quarter & Australia & Canada & Colombia & Indonesia & Malaysia & Venezuela & $\begin{array}{l}\text { Other } \\
\text { Countries }\end{array}$ & Total \\
\hline & \multicolumn{8}{|c|}{ Quantity } \\
\hline 1992 & 101 & 1,021 & 1,763 & 253 & 53 & 539 & 72 & 3,803 \\
\hline 1993 & 100 & 1,051 & 4,117 & 708 & - & 1,298 & 34 & 7,309 \\
\hline 1994 & 92 & 1,253 & 3,390 & 1,130 & - & 1,531 & 188 & 7,584 \\
\hline 1995 & 212 & 1,320 & 2,737 & 1,018 & - & 1,846 & 68 & 7,201 \\
\hline 1996 & 165 & 1,427 & 2,527 & 1,535 & - & 1,463 & 10 & 7,126 \\
\hline \multicolumn{9}{|l|}{1997} \\
\hline January - March & 54 & 181 & 492 & 396 & - & 147 & 61 & 1,331 \\
\hline April - June & 30 & 318 & 875 & 187 & - & 290 & 8 & 1,708 \\
\hline July - September.............................. & - & 308 & 1,042 & 394 & - & 455 & 23 & $\mathbf{2 , 2 2 2}$ \\
\hline October - December ...................... & 31 & 406 & 708 & 449 & - & 623 & 9 & 2,226 \\
\hline Total & 116 & 1,212 & 3,117 & 1,426 & - & 1,514 & 103 & $\mathbf{7 , 4 8 7}$ \\
\hline \multicolumn{9}{|l|}{1998} \\
\hline January - March & 32 & 131 & 659 & 246 & _- & 767 & 5 & 1,839 \\
\hline April - June & - & 298 & 844 & 403 & - & 646 & 2 & 2,193 \\
\hline \multirow[t]{2}{*}{ 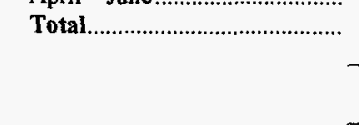 } & 32 & 429 & 1,503 & 649 & - & 1,412 & 7 & 4,031 \\
\hline & \multicolumn{8}{|c|}{ Average Price } \\
\hline 1992 & $\$ 36.07$ & $\$ 27.88$ & $\$ 32.25$ & $\$ 40.94$ & $\$ 47.06$ & $\$ 35.61$ & $\$ 25.72$ & $\$ 32.48$ \\
\hline 1993 & 31.56 & 29.02 & 27.26 & 42.70 & - & 28.87 & 26.22 & 29.36 \\
\hline 1994 & 30.02 & 30.61 & 27.46 & 33.80 & - & 32.41 & 29.33 & 29.98 \\
\hline 1995 & 30.99 & 32.59 & 31.15 & 35.13 & - & 35.14 & 46.29 & 33.11 \\
\hline 1996 & 33.41 & 32.86 & 31.40 & 32.45 & - & 30.97 & 33.43 & 31.82 \\
\hline \multicolumn{9}{|l|}{1997} \\
\hline January - March ............................. & 33.80 & 26.87 & 31.98 & 31.82 & - & 31.64 & - & 31.32 \\
\hline April - June .............................. & 34.95 & 39.87 & 32.64 & 36.48 & - & 33.23 & - & $\mathbf{3 4 . 4 8}$ \\
\hline July - September & - & 37.67 & 32.38 & 32.04 & - & 32.01 & 49.09 & 33.02 \\
\hline October - December ………............ & 31.45 & 38.18 & 31.17 & 32.86 & - & 34.57 & 49.22 & 33.64 \\
\hline Total & 33.47 & 36.80 & 32.11 & 32.82 & - & 33.26 & 49.12 & 33.25 \\
\hline \multicolumn{9}{|l|}{1998} \\
\hline January - March & 31.35 & 33.66 & 33.01 & 38.77 & - & 32.36 & 42.03 & 33.54 \\
\hline 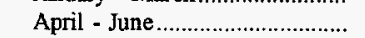 & - & 33.83 & 30.42 & 32.87 & - & 29.48 & - & 31.04 \\
\hline 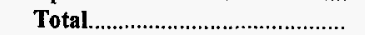 & 31.35 & 33.78 & 31.55 & $\mathbf{3 5 . 1 0}$ & - & 31.05 & 42.03 & 32.18 \\
\hline
\end{tabular}

Notes: Total may not equal sum of components because of independent rounding. Average price is based on the customs import value. Beginning in 1989, the average prices presented in this table are representative prices for coal imports that fall within the range of $\$ 20$ and $\$ 55$, inclusively. Therefore, the Total price column in this table will not equal theU.S. Total prices in Table Al. Coal imports include coal to Puerto Rico and the Virgin Islands. Source: Bureau of the Census, U.S. Department of Commerce, "Monthly Report IM 145." 
Table A3. U. S. Coal Imports by Origin and by Customs District (Short Tons)

\begin{tabular}{|c|c|c|c|c|c|c|}
\hline \multirow[b]{2}{*}{ Customs District } & \multirow{2}{*}{$\begin{array}{l}\text { April - } \\
\text { June } \\
1998\end{array}$} & \multirow{2}{*}{$\begin{array}{c}\text { January - } \\
\text { March } \\
1998\end{array}$} & \multirow{2}{*}{$\begin{array}{l}\text { April - } \\
\text { June } \\
1997\end{array}$} & \multicolumn{3}{|c|}{ Year to Date } \\
\hline & & & & 1998 & 1997 & $\begin{array}{l}\text { Percent } \\
\text { Change }\end{array}$ \\
\hline \multirow[t]{2}{*}{ U.S. Total ............................. } & $2,192,506$ & $1,838,994$ & $1,707,583$ & $4,031,500$ & $3,038,973$ & 32.7 \\
\hline & \multicolumn{6}{|c|}{ Exporting Country: Australia } \\
\hline \multirow{3}{*}{$\begin{array}{l}\text { Honolulu, HI } \\
\text { Total }\end{array}$} & - & 31,523 & 30,307 & 31,523 & 84,458 & -62.7 \\
\hline & - & 31,523 & 30,307 & 31,523 & 84,458 & -62.7 \\
\hline & \multicolumn{6}{|c|}{ Exporting Country: Canada } \\
\hline Chicago, IL ............................ & 33,746 & - & 92,757 & 33,746 & 92,757 & -63.6 \\
\hline 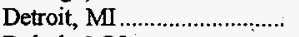 & 63,314 & 34,814 & 125,882 & 98,128 & 131,353 & -25.3 \\
\hline Duluth, MN & - & - & - & - & 416 & - \\
\hline Great Falls, MT ....................... & - & - & 281 & - & 281 & - \\
\hline 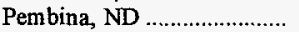 & 175,340 & 69,514 & 82,918 & 244,854 & 230,118 & 6.4 \\
\hline 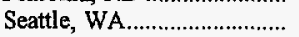 & 25,354 & 27,131 & 15,724 & 52,485 & 43,151 & 21.6 \\
\hline \multirow[t]{2}{*}{ 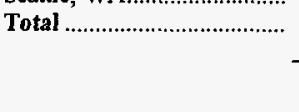 } & 297,754 & 131,459 & 317,562 & 429,213 & 498,076 & -13.8 \\
\hline & \multicolumn{6}{|c|}{ Exporting Country: Colombia } \\
\hline Mobile, AL ............................. & 197,953 & - & 34,975 & 197,953 & 98,865 & 100.2 \\
\hline 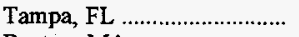 & 298,011 & 343,005 & 425,655 & 641,016 & 564,554 & 13.5 \\
\hline Boston, MA & 180,737 & 204,700 & 329,688 & 385,437 & 528,739 & -27.1 \\
\hline 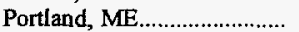 & - & - & 5,622 & - & 34,154 & - \\
\hline New York City, NY .............. & 50,324 & 77,571 & 39,048 & 127,895 & 73,149 & 74.8 \\
\hline Portland, OR ......................... & 6,992 & - & - & 6,992 & - & - \\
\hline 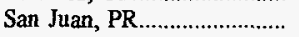 & 53,749 & 27,293 & 39,739 & 81,042 & 67,753 & 19.6 \\
\hline Houston-Galveston, TX........ & - & 6,068 & - & 6,068 & - & - \\
\hline 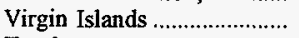 & 56,444 & - & - & 56,444 & - & - \\
\hline \multirow[t]{2}{*}{ Total } & 844,210 & 658,637 & 874,727 & $1,502,847$ & $1,367,214$ & 9.9 \\
\hline & \multicolumn{6}{|c|}{ Exporting Country: Indonesia } \\
\hline Honolulu, HI.......................... & 179,822 & 171,811 & 118,674 & $351, \overline{633}$ & 295,571 & 19.0 \\
\hline New Orleans, LA...................... & 223,471 & 73,836 & 68,242 & 297,307 & 287,051 & 3.6 \\
\hline \multirow[t]{2}{*}{ 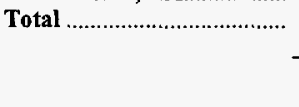 } & 403,293 & 245,647 & 186,916 & 648,940 & 582,622 & 11.4 \\
\hline & \multicolumn{6}{|c|}{ Exporting Country: Venezuela } \\
\hline Mobile, AL ............................ & 158,898 & 97,266 & - & 256,164 & - & - \\
\hline 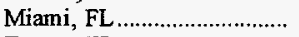 & 45,093 & 45,288 & - & 90,381 & - & - \\
\hline 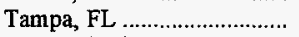 & 42,452 & - & - & 42,452 & - & - \\
\hline 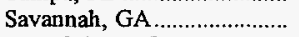 & 63,824 & 76,061 & 43,120 & $139 ; 885$ & 68,920 & 103.0 \\
\hline New Orleans, LA................... & - & 79,765 & - & 79,765 & - & - \\
\hline Boston, MA & 47,497 & 261,219 & 67,615 & 308,716 & 95,443 & 223.5 \\
\hline 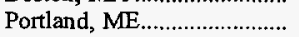 & 116,021 & 34,681 & 63,636 & 150,702 & 121,814 & 23.7 \\
\hline 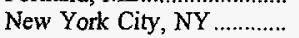 & 54,518 & 79,796 & 66,459 & 134,314 & 101,727 & 32.0 \\
\hline Philadelphia, PA & - & 64,354 & 27,226 & 64,354 & 27,226 & 136.4 \\
\hline San Juan, PR & - & 28,385 & 21,605 & 28,385 & 21,605 & 31.4 \\
\hline Houston-Galveston, TX....... & 117,290 & - & - & 117,290 & - & - \\
\hline \multirow[t]{2}{*}{ 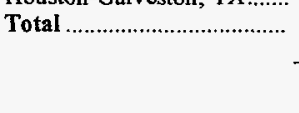 } & 645,593 & 766,815 & 289,661 & $1,412,408$ & 436,735 & 223.4 \\
\hline & \multicolumn{6}{|c|}{ Other Exporting Countries } \\
\hline Los Angeles, CA .................... & - & - & - & - & 149 & - \\
\hline 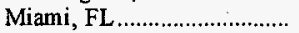 & - & - & 13 & - & 13 & - \\
\hline 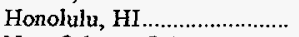 & - & - & 1 & - & 1 & - \\
\hline New Orleans, LA................... & - & - & - & - & 41,005 & - \\
\hline 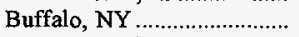 & - & 1,847 & 1,241 & 1,847 & 2,479 & -25.5 \\
\hline New York City, NY ............... & - & 25 & - & 25 & - & - \\
\hline Portland, OR & - & - & 6,852 & - & 13,441 & - \\
\hline 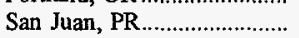 & 437 & 2,226 & - & 2,663 & - & - \\
\hline Houston-Galveston, TX....... & - & - & - & - & 12,124 & - \\
\hline Laredo, TX & 694 & - & - & 694 & 353 & 96.6 \\
\hline 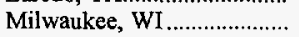 & 525 & 815 & 303 & 1,340 & 303 & 342.2 \\
\hline Total & 1,656 & 4,913 & 8,410 & 6,569 & 69,868 & -90.6 \\
\hline
\end{tabular}

Note: Total may not equal sum of components because of independent rounding.

Source: Bureau of the Census, U.S. Department of Commerce, "Monthly Report IM 145." 
Table A4. Average Price of U.S. Coal Imports by Origin and by Customs District (Dollars per Short Ton)

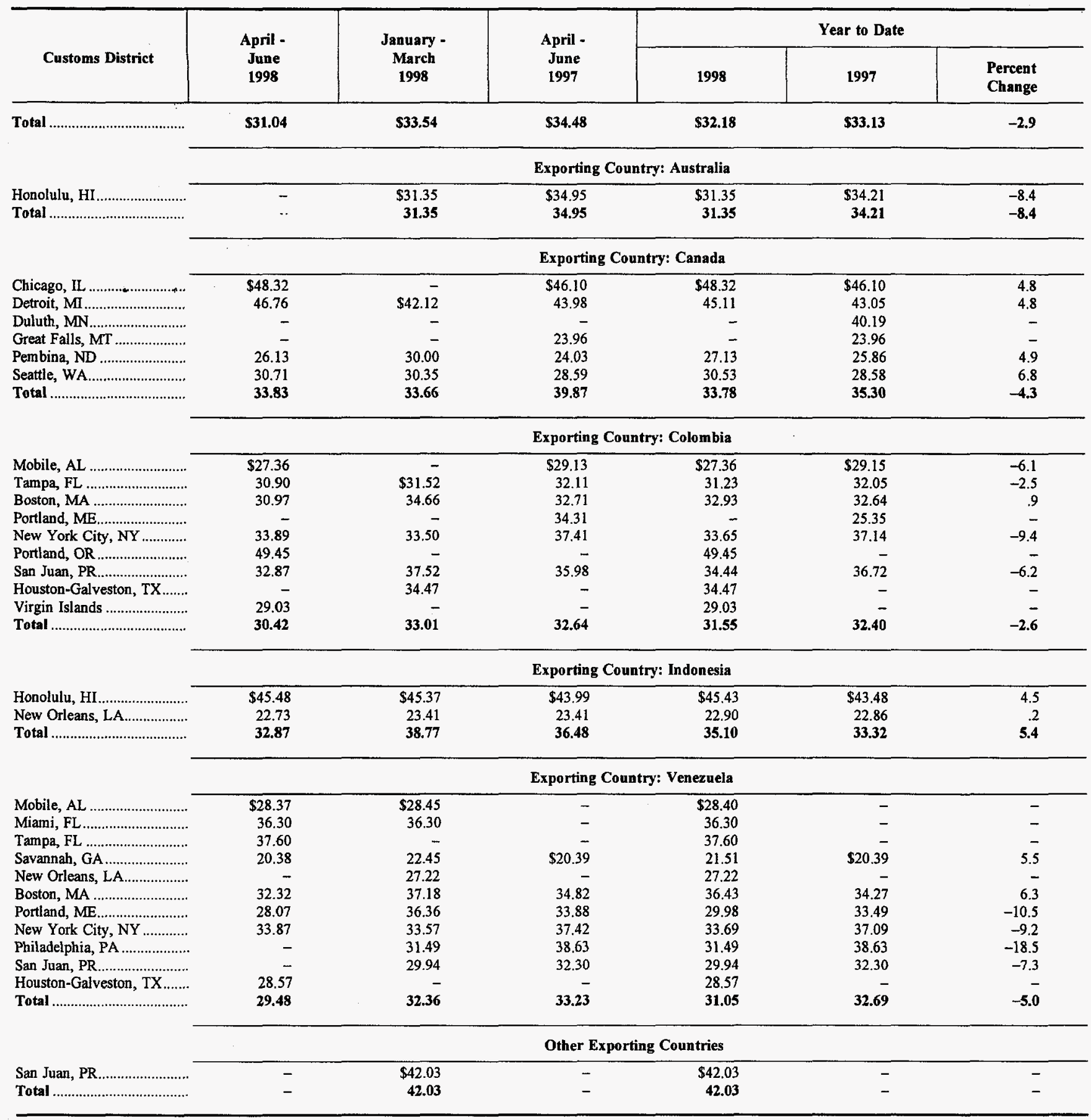

Notes: Total may not equal sum of components because of independent rounding. Average price is based on the customs import value. Beginning in 1989 , the average prices presented in this table are representative prices for coal imports that fall within the range of $\$ 20$ and $\$ 55$, inclusively. Therefore, the Total price column in this table will not equal theU.S. Total prices in Table A1.

Source: Bureau of the Census, U.S. Department of Commerce, "Monthly Report IM 145." 
Table A5. Imported Coal Received at Electric Utility Plants by Origin (Short Tons)

\begin{tabular}{|c|c|c|c|c|c|c|}
\hline \multirow[b]{2}{*}{ Company and Plant } & \multirow{2}{*}{$\begin{array}{l}\text { April - } \\
\text { June } \\
1998\end{array}$} & \multirow{2}{*}{$\begin{array}{l}\text { January - } \\
\text { March } \\
1998\end{array}$} & \multirow{2}{*}{$\begin{array}{l}\text { April - } \\
\text { June } \\
1997\end{array}$} & \multicolumn{3}{|c|}{ Year to Date } \\
\hline & & & & 1998 & 1997 & $\begin{array}{l}\text { Percent } \\
\text { Change }\end{array}$ \\
\hline \multirow[t]{2}{*}{ 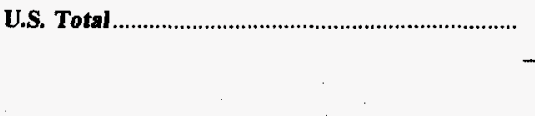 } & $1,257,325$ & $1,418,240$ & $1,138,848$ & $2,675,565$ & $1,862,796$ & 43.6 \\
\hline & \multicolumn{6}{|c|}{ Exporting Country: Canada } \\
\hline \multirow{3}{*}{$\begin{array}{l}\text { Takoma Dept, of Public Utilities, Steam No.2 } \\
\text { Total }\end{array}$} & - & - & - & - & 4,370 & - \\
\hline & - & - & - & - & 4,370 & - \\
\hline & \multicolumn{6}{|c|}{ Exporting Country: Colombia } \\
\hline Central Hudson Gas \& Eleetric, Dańskammer & - & 34,800 & - & 34,800 & - & - \\
\hline $\begin{array}{l}\text { Central Power and Light (CSW), Coleto Creek } \\
\text { City Public Service, San Antonio JT }\end{array}$ & 23,720 & - & - & 23,720 & - & - \\
\hline Deely/Spruce & - & 24,000 & - & 24,000 & - & - \\
\hline Gulf Power, Crist & 43,616 & - & - & 43,616 & - & - \\
\hline Jacksonville Electric Authority, St Johns River & 296,820 & 359,270 & 425,690 & 656,090 & 564,610 & 16.2 \\
\hline New England Power (NEES), Brayton Point ............ & 71,400 & 151,400 & 159,300 & 222,800 & 271,400 & -17.9 \\
\hline New England Power (NEES), Salem Harbor............ & 87,500 & 76,700 & 153,900 & 164,200 & 239,500 & -31.4 \\
\hline \multirow{3}{*}{ 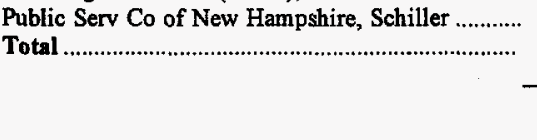 } & & 34,680 & - & 34,680 & - & - \\
\hline & 523,056 & 680,850 & 738,890 & $1,203,906$ & $1, \mathbf{0 7 5 , 5 1 0}$ & 11.9 \\
\hline & \multicolumn{6}{|c|}{ Exporting Country: Indonesia } \\
\hline \multirow{3}{*}{$\begin{array}{l}\text { Tampa Electric, Davant Transfer } \\
\text { Total }\end{array}$} & 223,472 & 73,835 & 68,241 & 297,307 & 287,048 & 3.6 \\
\hline & 223,472 & 73,835 & 68,241 & 297,307 & 287,048 & 3.6 \\
\hline & \multicolumn{6}{|c|}{ Exporting Country: Venezuela } \\
\hline $\begin{array}{l}\text { Central Hudson Gas \& Electric, Danskammer } \\
\text { City Public Service, San Antonio JT }\end{array}$ & 104,900 & 122,600 & 88,350 & 227,500 & 157,750 & 44.2 \\
\hline Deely/Spruce & 9,000 & - & - & 9,000 & - & - \\
\hline Florida Power Corp, IMT Transfer............................ & 18,547 & 61,217 & - & 79,764 & - & - \\
\hline 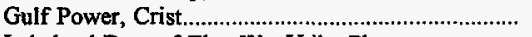 & 52,778 & 54,180 & - & 106,958 & - & - \\
\hline \multicolumn{7}{|l|}{ Lakeland Dept of Elec Wtr Utils, Plant } \\
\hline 3-Mcintosh & 6,000 & - & - & 6,000 & - & - \\
\hline Mississippi Power (Southern Co), Watson ............... & 51,070 & 13,600 & - & 64,670 & - & - \\
\hline New England Power (NEES), Brayton Point........... & 33,500 & 119,200 & 83,900 & 152,700 & 111,700 & 36.7 \\
\hline New England Power (NEES), Salem Harbor............ & 53,800 & 100,900 & - & 154,700 & - & - \\
\hline Public Serv Co of New Hampshire, Schiller ............ & 73,827 & 73,906 & 98,887 & 147,733 & 127,419 & 15.9 \\
\hline Public Serv Electric \& Gas Co, Mercer ..................... & - & 39,000 & - & 39,000 & - & - \\
\hline Savannah Electric and Power, Port Wentworth & 71,375 & 78,952 & 60,580 & 150,327 & 98,999 & 51.8 \\
\hline United Illuminating Co, Bridgeport Harbor ............... & 36,000 & - & - & 36,000 & - & - \\
\hline Total & 510,797 & 663,555 & 331,717 & $1,174,352$ & 495,868 & 136.8 \\
\hline
\end{tabular}

Note: Total may not equal sum of components because of independent rounding.

Source: Federal Energy Regulatory Commission, FERC Form 423, "Monthly Report of Cost and Quality of Fuels for Electric Plants." 
Table A6. Cost and Quality of Imported Coal Received at Electric Utility Plants by Origin, 1992-1998

\begin{tabular}{|c|c|c|c|c|c|c|}
\hline \multirow[b]{2}{*}{ Exporting Country and Time Period } & \multirow{2}{*}{$\begin{array}{l}\text { Quantity } \\
\text { (thousand } \\
\text { short tons) }\end{array}$} & \multicolumn{3}{|c|}{ Average Quality 1} & \multicolumn{2}{|c|}{ Average Cost Delivered } \\
\hline & & $\begin{array}{c}\text { Btu } \\
\text { per Pound }\end{array}$ & $\begin{array}{c}\text { Sulfur } \\
\text { Percent } \\
\text { by Weight }\end{array}$ & $\begin{array}{c}\text { Ash } \\
\text { Percent } \\
\text { by Weight }\end{array}$ & $\begin{array}{l}\text { Cents per } \\
\text { Million Btu }\end{array}$ & $\begin{array}{l}\text { Dollars per } \\
\text { Short Ton }\end{array}$ \\
\hline \multicolumn{7}{|c|}{ Company and Plant: Baltimore Gas and Electric, Brandon Shores } \\
\hline \multicolumn{7}{|l|}{ Colombia } \\
\hline 1993 & 224.0 & 12,354 & 0.64 & 6.32 & 149.8 & 37.02 \\
\hline \multirow[t]{2}{*}{ 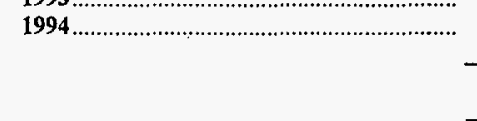 } & 88.0 & 12,379 & .66 & 7.36 & 147.3 & 36.46 \\
\hline & \multicolumn{6}{|c|}{ Company and Plant: Cajun Electric Power Coop, Big Cajun No. 2} \\
\hline \multicolumn{7}{|l|}{ Indonesia } \\
\hline 1994 & 169.2 & 9,702 & 0.10 & 1.20 & 166.8 & 32.36 \\
\hline & & Compa & d Plant: Caro & wer and Lig & & \\
\hline \multicolumn{7}{|l|}{ Colombia } \\
\hline \multirow{2}{*}{ 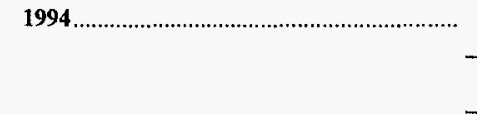 } & 26.6 & 12,200 & 0.70 & 9.00 & 145.5 & 35.50 \\
\hline & \multicolumn{6}{|c|}{ Company and Plant: Central Hudson Gas \& Electric, Danskammer } \\
\hline Colombia & & & & & & \\
\hline \multicolumn{7}{|l|}{1997} \\
\hline 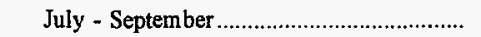 & 66.8 & 12,995 & 0.67 & 7.29 & 173.4 & 45.06 \\
\hline 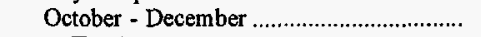 & 80.2 & 13,062 & .63 & 7.07 & 169.5 & 44.29 \\
\hline 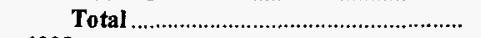 & 147.0 & 13,032 & .65 & 7.17 & 171.3 & 44.64 \\
\hline \multicolumn{7}{|l|}{1998} \\
\hline January - March & 34.8 & 13,309 & .62 & 7.38 & 169.8 & 45.20 \\
\hline 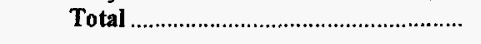 & 34.8 & 13,309 & .62 & 7.38 & 169.8 & 45.20 \\
\hline \multicolumn{7}{|l|}{ Venezuela } \\
\hline $1995 \ldots \ldots \ldots \ldots \ldots \ldots \ldots$ & 28.2 & 13,281 & .56 & 7.30 & 224.1 & 59.53 \\
\hline \multicolumn{7}{|l|}{1997} \\
\hline January - March & 69.4 & 13,181 & .66 & 6.35 & 174.9 & 46.11 \\
\hline 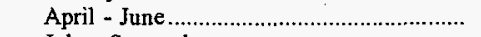 & 88.3 & 13,416 & .64 & 5.45 & 174.0 & 46.69 \\
\hline 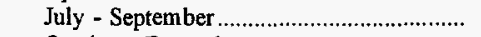 & 101.4 & 13,078 & .66 & 6.64 & 173.8 & 45.47 \\
\hline October - December ..................................... & 91.2 & 13,033 & .64 & 7.09 & 170.1 & 44.33 \\
\hline 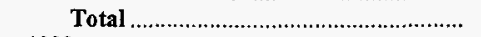 & 350.4 & 13,172 & .65 & 6.40 & 173.1 & 45.61 \\
\hline \multicolumn{7}{|l|}{1998} \\
\hline January - March & 122.6 & 13,311 & .59 & 7.02 & 169.8 & 45.21 \\
\hline 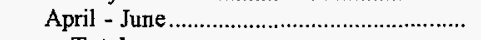 & 104.9 & 13,184 & .65 & 7.70 & 170.0 & 44.82 \\
\hline \multirow[t]{2}{*}{ Total } & 227.5 & 13,252 & .62 & 7.33 & 169.9 & 45.03 \\
\hline & \multicolumn{6}{|c|}{ Company and Plant: City Public Service, San Antonio JT Deely/Spruce } \\
\hline \multirow{2}{*}{\multicolumn{7}{|c|}{$\begin{array}{l}\text { Colombia } \\
1997\end{array}$}} \\
\hline & & & & & & \\
\hline 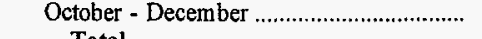 & 73.0 & 11,603 & 0.34 & 3.89 & 176.9 & 41.06 \\
\hline 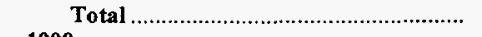 & $\mathbf{7 3 . 0}$ & 11,603 & .34 & 3.89 & 176.9 & 41.06 \\
\hline 1998 & & & & & & \\
\hline January - March & 24.0 & 11,600 & .33 & 3.80 & 200.6 & 46.54 \\
\hline 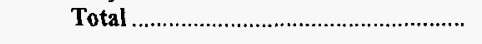 & 24.0 & 11,600 & .33 & 3.80 & 200.6 & 46.54 \\
\hline $\begin{array}{l}\text { Venezuela } \\
1998\end{array}$ & & & & & & \\
\hline 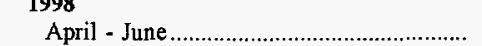 & 9.0 & 12,855 & .70 & & 185.8 & 47.77 \\
\hline Total & 9.0 & 12,855 & .70 & 6.00 & 185.8 & 47.77 \\
\hline & & Company an & It: Central Po & d Light (CSV & eto Creek & \\
\hline Colombia & & & & & & \\
\hline $1992 \ldots$ & 37.2 & 12,892 & 0.62 & 7.90 & 174.5 & 44.99 \\
\hline 1993 & 122.5 & 12,109 & .60 & 5.90 & 148.5 & 35.98 \\
\hline $1994 \ldots$ & 153.4 & 11,929 & .55 & 5.03 & 148.9 & 35.51 \\
\hline
\end{tabular}

See footnotes at the end of Table A6. 
Table A6. Cost and Quality of Imported Coal Received at Electric Utility Plants by Origin, 1992-1998 (Continued)

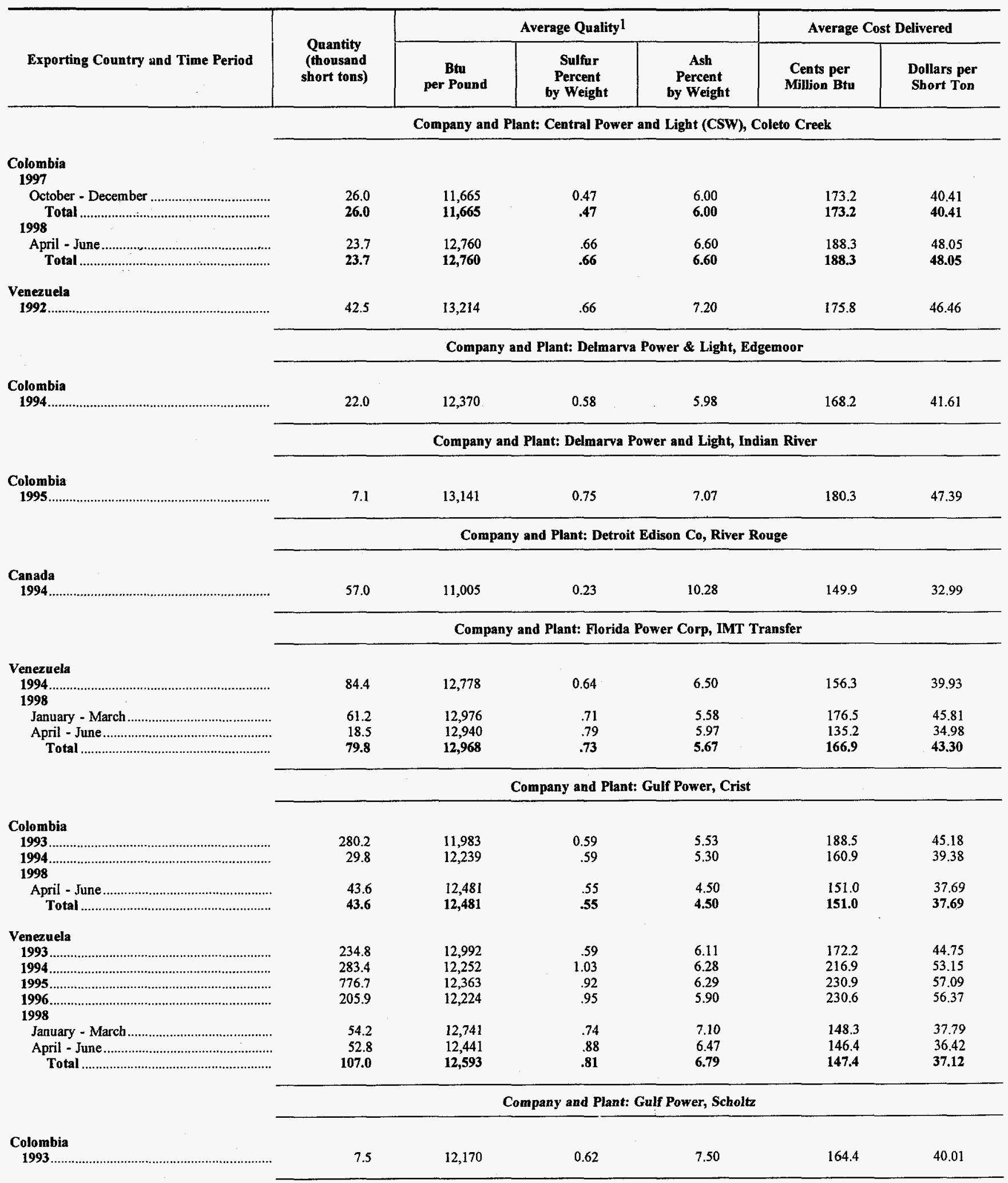

See footnotes at the end of Table A6. 
Table A6. Cost and Quality of Imported Coal Received at Electric Utility Plants by Origin, 1992-1998 (Continued)

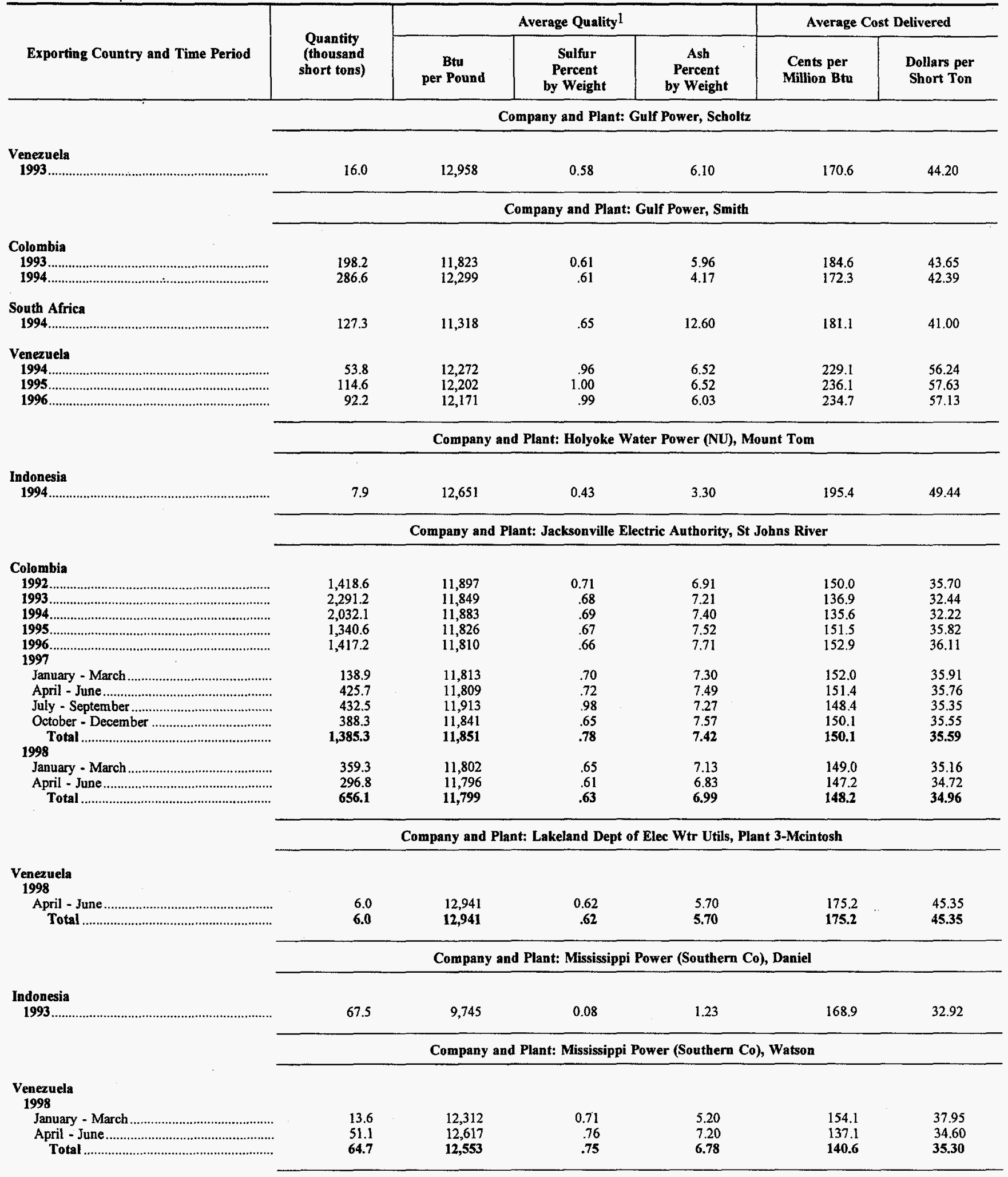

See footnotes at the end of Table A6. 
Table A6. Cost and Quality of Imported Coal Received at Electric Utility Plants by Origin, 1992-1998 (Continued)

\begin{tabular}{|c|c|c|c|c|c|c|}
\hline \multirow[b]{2}{*}{ Exporting Country and Time Period } & \multirow{2}{*}{$\begin{array}{l}\text { Quantity } \\
\text { (thousand } \\
\text { short tons) }\end{array}$} & \multicolumn{3}{|c|}{ Average Quality1 } & \multicolumn{2}{|c|}{ Average Cost Delivered } \\
\hline & & $\begin{array}{c}\text { Btu } \\
\text { per Pound }\end{array}$ & $\begin{array}{c}\text { Sulfur } \\
\text { Percent } \\
\text { by Weight }\end{array}$ & $\begin{array}{c}\text { Ash } \\
\text { Percent } \\
\text { by Weight }\end{array}$ & $\begin{array}{l}\text { Cents per } \\
\text { Million Btu }\end{array}$ & $\begin{array}{l}\text { Dollars per } \\
\text { Short Ton }\end{array}$ \\
\hline
\end{tabular}

Company and Plant: New England Power (NEES), Brayton Point

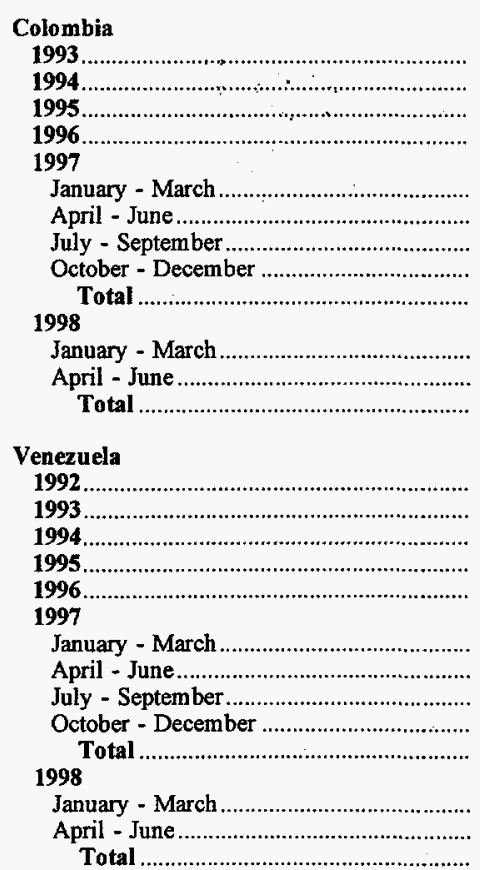

\section{2}

\section{3}

307.8

427.5

112.1

159.3
275.3

275.3
121.8

668.5

151.4

71.4

222.8

$\begin{array}{ll}129.0 & 13,375 \\ 239.9 & 13,132\end{array}$

239.9

351.2

510.6

572.4

$27.8 \quad 12,705$

83.9

110.7

70.1

292.5

119.2

33.5

152.7
12,144

12,131

12,218

12,014

12,030

12,190

12,093

12,169

12,119

12,123

12,128

12,125

12,955

12,788

12,937

13,136
13,203

13,143

13,122

13,270

13,063

13,225

0.64
.65
.60
.59

.63
.64
.63
.66
.64
.64
.64
.64

.60
.59

$63 \quad 6.04$

$\begin{array}{ll}.63 & 6.04 \\ .64 & 6.16\end{array}$

$\begin{array}{ll}.63 & 5.54 \\ 66 & 5.62\end{array}$

$\begin{array}{ll}.64 & 5.79\end{array}$

$64 \quad 6.25$

$\begin{array}{ll}.64 & 5.65 \\ 64 & 6.06\end{array}$

$.75 \quad 7.32$

$\begin{array}{ll}.71 & 7.83 \\ .71 & 7.03 \\ .69 & 7.03\end{array}$

$\begin{array}{ll}.67 & 6.29\end{array}$

$\begin{array}{ll}.75 & 8.48\end{array}$

$\begin{array}{ll}.75 & 8.48 \\ .72 & 6.23 \\ .65 & 5.58\end{array}$

$\begin{array}{ll}.65 & 5.58 \\ .67 & 6.86\end{array}$

$\begin{array}{ll}.68 & 6.35\end{array}$

$\begin{array}{ll}.65 & 7.33 \\ .66 & 8.96\end{array}$

$\begin{array}{ll}.66 & 8.96 \\ .65 & \mathbf{7 . 6 9}\end{array}$

\begin{tabular}{l}
5.42 \\
5.60 \\
5.22 \\
.52 \\
.04 \\
.16 \\
.54 \\
.62 \\
.79 \\
6.25 \\
5.65 \\
6.06 \\
\\
7.32 \\
.83 \\
.03 \\
.03 \\
6.29 \\
8.48 \\
6.23 \\
.58 \\
6.86 \\
7.35 \\
8.96 \\
7.69 \\
\hline
\end{tabular}

43.35

41.78

40.23

40.35

43.25

37.68

38.35

44.54

40.14

39.36

38.77

39.17

161.5

44.18

42.67

39.95

40.92

42.01

41.21

42.98

43.47

44.49

43.36

40.89

38.96

$\mathbf{4 0 . 4 7}$

Company and Plant: New England Power (NEES), Salem Harbor

Canada

Canada

\section{Colombia}

1994

1995.

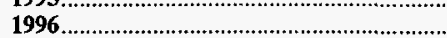

1997

January - March .........................................

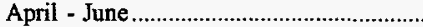

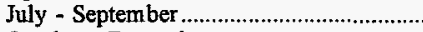

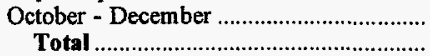

1998

January - March

April - June

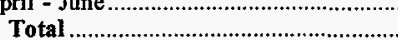

Venezuela

1992

1992

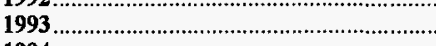

1994.

1995

1996

1997

October - December

Total

1998

January - March

April - June.

Total.

See footnotes at the end of Table A6. 
Table A6. Cost and Quality of Imported Coal Received at Electric Utility Plants by Origin, 1992-1998 (Continued)

\begin{tabular}{|c|c|c|c|c|c|c|}
\hline \multirow[b]{2}{*}{ Exporting Country and Time Period } & \multirow{2}{*}{$\begin{array}{c}\text { Quantity } \\
\text { (thousand } \\
\text { short tons) }\end{array}$} & \multicolumn{3}{|c|}{ Average Quality 1} & \multicolumn{2}{|c|}{ Average Cost Delivered } \\
\hline & & $\begin{array}{c}\text { Btu } \\
\text { per Pound }\end{array}$ & $\begin{array}{c}\text { Sulfur } \\
\text { Percent } \\
\text { by Weight }\end{array}$ & $\begin{array}{c}\text { Ash } \\
\text { Percent } \\
\text { by Weight }\end{array}$ & $\begin{array}{l}\text { Cents per } \\
\text { Million Btu }\end{array}$ & $\begin{array}{l}\text { Dollars per } \\
\text { Short Ton }\end{array}$ \\
\hline & \multicolumn{6}{|c|}{ Company and Plant: Ohio Edison, Burger Plant } \\
\hline \multicolumn{7}{|l|}{ Indonesia } \\
\hline \multirow{2}{*}{ 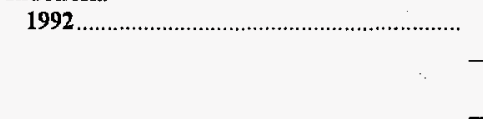 } & 13.1 & 9,587 & 0.14 & 1.20 & 166.9 & 32.00 \\
\hline & \multicolumn{6}{|c|}{ Company and Plant: Public Serv Co of Indiana, Gallagher } \\
\hline \multicolumn{7}{|l|}{ Indonesia } \\
\hline \multirow[t]{2}{*}{1993} & 11.1 & 9,242 & 0.13 & 1.35 & 104.8 & 19.38 \\
\hline & \multicolumn{6}{|c|}{ Company and Plant: Public Serv Co of New Hampshire, Merrimack } \\
\hline \multicolumn{7}{|l|}{ Colombia } \\
\hline $1995 \ldots \ldots$ & 11.5 & 11,578 & 0.53 & 3.80 & 192.9 & 44.67 \\
\hline \multicolumn{7}{|l|}{ Indonesia } \\
\hline 1993 & 21.2 & 12,620 & .49 & 3.80 & 186.5 & 47.07 \\
\hline \multicolumn{7}{|l|}{1997} \\
\hline 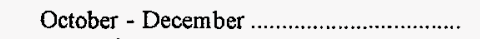 & 40.8 & 12,300 & .49 & 4.50 & 190.7 & 46.92 \\
\hline Total & 40.8 & 12,300 & .49 & 4.50 & 190.7 & 46.92 \\
\hline \multicolumn{7}{|l|}{ Venezuela } \\
\hline 1993 & 24.9 & 12,920 & .58 & 6.00 & 163.2 & 42.17 \\
\hline \multirow[t]{2}{*}{$1996 \ldots+\ldots$} & 39.9 & 12,370 & .39 & 3.70 & 213.2 & 52.75 \\
\hline & \multicolumn{6}{|c|}{ Company and Plant: Public Serv Co of New Hampshire, Schiller } \\
\hline \multicolumn{7}{|l|}{ Colombia } \\
\hline 1992 & 48.4 & 12,428 & 0.61 & 6.31 & 157.2 & 39.08 \\
\hline 1993 & 52.1 & 12,861 & .64 & 7.49 & 150.0 & 38.59 \\
\hline 1994 & 163.3 & 12,505 & .62 & 5.55 & 135.5 & 33.89 \\
\hline 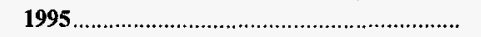 & 122.9 & 12,733 & .62 & 6.70 & 160.0 & 40.73 \\
\hline $1996 \ldots \ldots \ldots \ldots$ & 32.3 & 12,169 & .66 & 5.68 & 161.9 & 39.41 \\
\hline \multicolumn{7}{|l|}{1997} \\
\hline 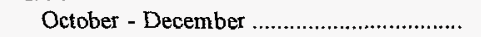 & 35.4 & 13,231 & .63 & 6.70 & 160.1 & 42.37 \\
\hline 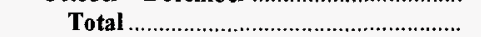 & 35.4 & 13,231 & .63 & 6.70 & 160.1 & 42.37 \\
\hline \multicolumn{7}{|l|}{1998} \\
\hline 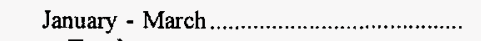 & 34.7 & 13,188 & .64 & 5.50 & 172.8 & 45.58 \\
\hline 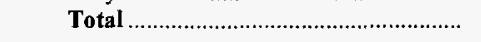 & 34.7 & 13,188 & .64 & 5.50 & 172.8 & 45.58 \\
\hline Indonesia & & & & & & \\
\hline 1993 & 16.0 & 12,620 & .49 & 3.80 & 161.3 & 40.71 \\
\hline 1994 & 113.0 & 12,360 & .53 & 3.58 & 158.7 & 39.23 \\
\hline 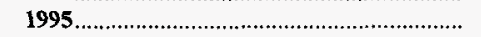 & 79.7 & 12,300 & .52 & 4.56 & 167.8 & 41.28 \\
\hline 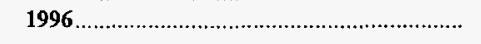 & 25.9 & 12,412 & .72 & 8.20 & 161.9 & 40.19 \\
\hline Venezuela & & & & & & \\
\hline 1992 & 34.3 & 12,881 & .58 & 6.76 & 168.0 & 43.29 \\
\hline 1993 & 84.3 & 12,972 & .58 & 6.08 & 138.6 & 35.95 \\
\hline 1995 & 82.4 & 13,044 & .71 & 7.24 & 156.5 & 40.84 \\
\hline $1996 \ldots \ldots \ldots$ & 56.1 & 13,061 & .67 & 6.04 & 159.8 & 41.75 \\
\hline 1997 & & & & & & \\
\hline 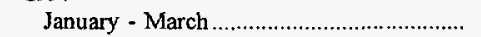 & 28.5 & 11,669 & .88 & 7.90 & 160.0 & 37.34 \\
\hline 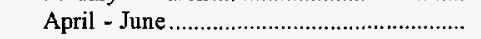 & 98.9 & 12,707 & .66 & 5.50 & 161.9 & 41.15 \\
\hline July - September & 101.5 & 11,893 & .63 & 6.24 & 159.7 & 38.00 \\
\hline Total & 229.0 & 12,217 & .67 & 6.13 & 160.7 & 39.27 \\
\hline 1998 & & & & & & \\
\hline 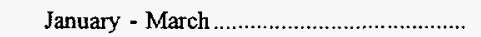 & 73.9 & 13,161 & .65 & 5.20 & 148.9 & 39.20 \\
\hline 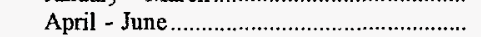 & 73.8 & 12,796 & .68 & 6.15 & 145.8 & 37.30 \\
\hline Total & 147.7 & 12,979 & .67 & 5.67 & 147.4 & 38.25 \\
\hline
\end{tabular}

See footnotes at the end of Table A6. 
Table A6. Cost and Quality of Imported Coal Received at Electric Utility Plants by Origin, 1992-1998 (Continued)

\begin{tabular}{|c|c|c|c|c|c|c|}
\hline \multirow[b]{2}{*}{ Exporting Country and Time Period } & \multirow{2}{*}{$\begin{array}{l}\text { Quantity } \\
\text { (thousand } \\
\text { short tons) }\end{array}$} & \multicolumn{3}{|c|}{ Average Qualityl } & \multicolumn{2}{|c|}{ Average Cost Delivered } \\
\hline & & $\begin{array}{c}\text { Btu } \\
\text { per Pound }\end{array}$ & $\begin{array}{l}\text { Sulfur } \\
\text { Pereent } \\
\text { by Weight }\end{array}$ & $\begin{array}{c}\text { Ash } \\
\text { Percent } \\
\text { by Weight }\end{array}$ & $\begin{array}{l}\text { Cents per } \\
\text { Million Btu }\end{array}$ & $\begin{array}{l}\text { Dollars per } \\
\text { Short Ton }\end{array}$ \\
\hline & \multicolumn{6}{|c|}{ Company and Plant: Public Serv Electric \& Gas Co, Mercer } \\
\hline \multicolumn{7}{|l|}{$\begin{array}{l}\text { Venezuela } \\
1998\end{array}$} \\
\hline \multirow[t]{2}{*}{ 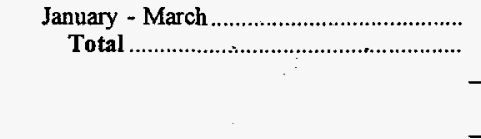 } & $\begin{array}{l}39.0 \\
39.0\end{array}$ & $\begin{array}{l}12,998 \\
12,998\end{array}$ & $\begin{array}{r}0.68 \\
.68\end{array}$ & $\begin{array}{l}5.50 \\
5.50\end{array}$ & $\begin{array}{l}155.3 \\
155.3\end{array}$ & $\begin{array}{l}40.37 \\
\mathbf{4 0 . 3 7}\end{array}$ \\
\hline & \multicolumn{6}{|c|}{ Company and Plant: Public Serv Electric \& Gas-NJ, Hudson } \\
\hline \multirow[t]{2}{*}{ 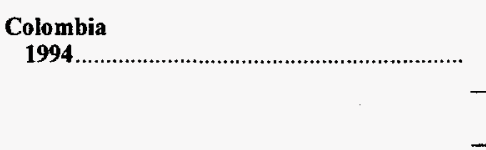 } & 22.5 & 12,870 & 0.68 & 6.90 & 166.9 & 42.96 \\
\hline & \multicolumn{6}{|c|}{ Company and Plant: Savannah Electric and Power, Port Wentworth } \\
\hline \multicolumn{5}{|l|}{ Colombia } & & 48.12 \\
\hline $\begin{array}{l}\text { Venezuela } \\
1994 \ldots \\
1996\end{array}$ & $\begin{array}{r}16.8 \\
209.9\end{array}$ & $\begin{array}{l}12,575 \\
12,143\end{array}$ & $\begin{array}{l}1.12 \\
1.08\end{array}$ & $\begin{array}{l}8.60 \\
6.71\end{array}$ & $\begin{array}{l}168.0 \\
152.8\end{array}$ & $\begin{array}{l}42.25 \\
37.11\end{array}$ \\
\hline 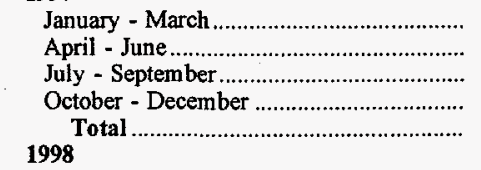 & $\begin{array}{r}38.4 \\
60.6 \\
130.3 \\
49.8 \\
279.1\end{array}$ & $\begin{array}{l}11,867 \\
11,867 \\
12,115 \\
11,682 \\
11,949\end{array}$ & $\begin{array}{r}1.60 \\
1.58 \\
1.20 \\
.85 \\
1.28\end{array}$ & $\begin{array}{l}8.20 \\
8.20 \\
7.64 \\
6.94 \\
7.72\end{array}$ & $\begin{array}{l}136.6 \\
138.1 \\
139.0 \\
119.9 \\
135.1\end{array}$ & $\begin{array}{l}32.42 \\
32.79 \\
33.67 \\
28.01 \\
32.29\end{array}$ \\
\hline \multirow{2}{*}{$\begin{array}{l}1998 \\
\text { January - March } \\
\text { April - June } \\
\quad \text { Total }\end{array}$} & $\begin{array}{r}79.0 \\
71.4 \\
150.3\end{array}$ & $\begin{array}{l}13,018 \\
11,986 \\
12,528\end{array}$ & $\begin{array}{r}.67 \\
1.58 \\
1.10\end{array}$ & $\begin{array}{l}5.50 \\
8.20 \\
6.78\end{array}$ & $\begin{array}{l}152.4 \\
147.8 \\
150.3\end{array}$ & $\begin{array}{l}39.68 \\
35.43 \\
37.66\end{array}$ \\
\hline & \multicolumn{6}{|c|}{ Company and Plant: Takoma Dept. of Public Utilities, Steam No.2 } \\
\hline \multirow[t]{2}{*}{$\begin{array}{l}\text { Canada } \\
1992 \ldots \ldots \\
1993 \\
1994 \\
1995 \\
1996 \\
1997 \\
\text { January - March } \\
\text { July - September } \\
\text { Total }\end{array}$} & $\begin{array}{r}15.3 \\
29.2 \\
6.3 \\
23.8 \\
18.0 \\
\\
4.4 \\
5.2 \\
9.6\end{array}$ & $\begin{array}{r}9,993 \\
10,036 \\
9,806 \\
10,066 \\
9,861 \\
\\
9,979 \\
10,283 \\
10,144\end{array}$ & $\begin{array}{r}0.42 \\
.48 \\
.48 \\
.47 \\
.44 \\
\\
.36 \\
.48 \\
.43\end{array}$ & $\begin{array}{l}12.95 \\
12.60 \\
12.80 \\
13.14 \\
12.97 \\
\\
12.97 \\
11.65 \\
12.25\end{array}$ & $\begin{array}{l}214.7 \\
179.5 \\
178.0 \\
166.0 \\
174.6 \\
\\
172.0 \\
171.0 \\
171.4\end{array}$ & $\begin{array}{l}42.90 \\
36.03 \\
34.91 \\
33.42 \\
34.44 \\
\\
34.33 \\
35.17 \\
34.79\end{array}$ \\
\hline & \multicolumn{6}{|c|}{ Company and Plant: Tampa Electric, Davant Transfer } \\
\hline Colombia & 222.2 & \multicolumn{5}{|c|}{ Colombia } \\
\hline \multicolumn{7}{|l|}{ Indonesia } \\
\hline $1994 \ldots$ & $\begin{array}{l}147.2 \\
348.9 \\
807.8\end{array}$ & $\begin{array}{l}9,871 \\
9,696 \\
9,655\end{array}$ & $\begin{array}{l}.09 \\
.31 \\
.29\end{array}$ & $\begin{array}{l}1.10 \\
1.16 \\
1.48\end{array}$ & $\begin{array}{l}143.0 \\
143.8 \\
149.7\end{array}$ & $\begin{array}{l}28.24 \\
27.88 \\
28.91\end{array}$ \\
\hline 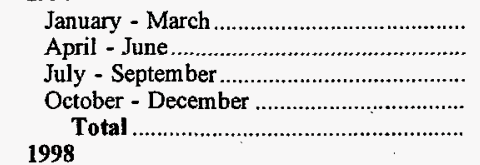 & $\begin{array}{r}218.8 \\
68.2 \\
219.9 \\
234.3 \\
741.3\end{array}$ & $\begin{array}{l}9,482 \\
9,521 \\
9,736 \\
9,649 \\
9,614\end{array}$ & $\begin{array}{l}.37 \\
.32 \\
.35 \\
.34 \\
.35\end{array}$ & $\begin{array}{l}1.67 \\
1.50 \\
1.20 \\
1.43 \\
1.44\end{array}$ & $\begin{array}{l}161.3 \\
163.3 \\
163.3 \\
163.3 \\
162.7\end{array}$ & $\begin{array}{l}30.59 \\
31.10 \\
31.80 \\
31.52 \\
\mathbf{3 1 . 2 9}\end{array}$ \\
\hline 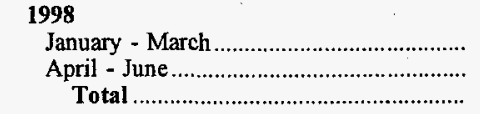 & $\begin{array}{r}73.8 \\
223.5 \\
297.3\end{array}$ & $\begin{array}{l}9,507 \\
9,623 \\
9,594\end{array}$ & $\begin{array}{l}.38 \\
.21 \\
.25\end{array}$ & $\begin{array}{l}1.10 \\
1.13 \\
1.12\end{array}$ & $\begin{array}{l}163.3 \\
157.9 \\
159.2\end{array}$ & $\begin{array}{l}31.05 \\
30.38 \\
\mathbf{3 0 . 5 5}\end{array}$ \\
\hline
\end{tabular}

See footnotes at the end of Table A6. 
Table A6. Cost and Quality of Imported Coal Received at Electric Utility Plants by Origin, 1992-1998 (Continued)

\begin{tabular}{|c|c|c|c|c|c|c|}
\hline \multirow[b]{2}{*}{ Exporting Country and Time Period } & \multirow{2}{*}{$\begin{array}{l}\text { Quantity } \\
\text { (thousand } \\
\text { short tons) }\end{array}$} & \multicolumn{3}{|c|}{ Average Quality 1} & \multicolumn{2}{|c|}{ Average Cost Delivered } \\
\hline & & $\begin{array}{c}\text { Btu } \\
\text { per Pound }\end{array}$ & $\begin{array}{c}\text { Sulfur } \\
\text { Percent } \\
\text { by Weight }\end{array}$ & $\begin{array}{c}\text { Ash } \\
\text { Percent } \\
\text { by Weight }\end{array}$ & $\begin{array}{l}\text { Cents per } \\
\text { Mtllion Bta }\end{array}$ & $\begin{array}{l}\text { Dollars per } \\
\text { Short Ton }\end{array}$ \\
\hline & \multicolumn{6}{|c|}{ Company and Plant: Tampa Electric, Davant Transfer } \\
\hline \multicolumn{7}{|l|}{ Venexuela } \\
\hline 1993 & 61.4 & 11,056 & 1.48 & 9.78 & 220.7 & 48.80 \\
\hline \multirow[t]{2}{*}{$\begin{array}{l}\text { October - December } \\
\text { Total }\end{array}$} & $\begin{array}{l}\mathbf{5 8 . 6} \\
\mathbf{5 8 . 6}\end{array}$ & $\begin{array}{l}12,953 \\
\mathbf{1 2 , 9 5 3}\end{array}$ & $\begin{array}{l}1.47 \\
1.47\end{array}$ & $\begin{array}{l}3.50 \\
\mathbf{3 . 5 0}\end{array}$ & $\begin{array}{l}130.2 \\
130.2\end{array}$ & $\begin{array}{l}33.73 \\
33.73\end{array}$ \\
\hline & \multicolumn{6}{|c|}{ Company and Plant: United Illuminating Co, Bridgeport Harbor } \\
\hline \multicolumn{7}{|l|}{ Venezuela } \\
\hline 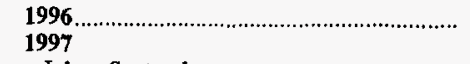 & 28.0 & 13,174 & 0.61 & 4.10 & 185.0 & 48.74 \\
\hline $\begin{array}{l}\text { July - September } \\
\text { Total } \\
\mathbf{1 9 9 8}\end{array}$ & $\begin{array}{l}35.0 \\
35.0\end{array}$ & $\begin{array}{l}13,387 \\
13,387\end{array}$ & $\begin{array}{l}.64 \\
.64\end{array}$ & $\begin{array}{l}4.30 \\
4.30\end{array}$ & $\begin{array}{l}169.6 \\
169.6\end{array}$ & $\begin{array}{l}45.41 \\
45.41\end{array}$ \\
\hline \multirow[t]{2}{*}{ 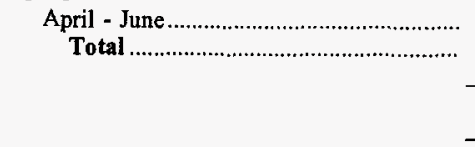 } & $\begin{array}{l}36.0 \\
36.0\end{array}$ & $\begin{array}{r}13,677 \\
13,677\end{array}$ & $\begin{array}{l}.58 \\
.58\end{array}$ & $\begin{array}{l}4.00 \\
4.00\end{array}$ & $\begin{array}{l}161.6 \\
161.6\end{array}$ & $\begin{array}{l}44.20 \\
44.20\end{array}$ \\
\hline & \multicolumn{6}{|c|}{ Total of U.S. Electric Utility Plants } \\
\hline \multicolumn{7}{|l|}{ Canada } \\
\hline 1992 & 48.1 & 12,432 & 1.09 & 6.72 & 185.1 & 46.01 \\
\hline 1993 & 29.2 & 10,036 & .48 & 12.60 & 179.5 & 36.03 \\
\hline 1994 & 63.3 & 10,885 & .26 & 10.53 & 152.4 & 33.19 \\
\hline 1995 & 23.8 & 10,066 & .47 & 13.14 & 166.0 & 33.42 \\
\hline $1996 \ldots \ldots \ldots$ & 18.0 & 9,861 & .44 & 12.97 & 174.6 & 34.44 \\
\hline January - March & 4.4 & 9,979 & .36 & 12.97 & 172.0 & 34.33 \\
\hline 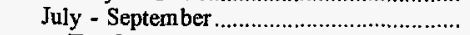 & 5.2 & 10,283 & .48 & 11.65 & 171.0 & 35.17 \\
\hline Total & 9.6 & 10,144 & .43 & 12.25 & 171.4 & 34.79 \\
\hline \multicolumn{7}{|l|}{ Colombia } \\
\hline 1992 & $1,504.1$ & 11,938 & .70 & 6.91 & 150.9 & 36.04 \\
\hline 1993 & $3,585.1$ & 11,867 & .66 & 6.85 & 149.0 & 35.37 \\
\hline 1994 & $2,971.8$ & 11,997 & .66 & 6.76 & 142.7 & 34.25 \\
\hline 1995 & $2,040.1$ & 11,985 & .65 & 6.83 & 153.9 & 36.89 \\
\hline 1996 & $2,079.9$ & 11,883 & .64 & 7.04 & 155.7 & 37.01 \\
\hline \multicolumn{7}{|l|}{1997} \\
\hline 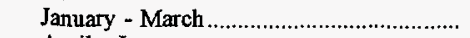 & 336.6 & 11,964 & .68 & 6.72 & 167.7 & 40.12 \\
\hline 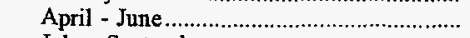 & 738.9 & 11,947 & .68 & 6.94 & 152.1 & 36.35 \\
\hline 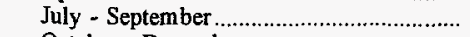 & 907.5 & 12,077 & .79 & 6.54 & 158.8 & 38.36 \\
\hline October - December & 761.4 & 12,070 & .61 & 6.68 & 161.2 & 38.90 \\
\hline 1998 & $2,744.4$ & 12,026 & .70 & 6.71 & 158.8 & 38.18 \\
\hline 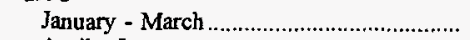 & 680.8 & 12,049 & .63 & 6.61 & 160.8 & 38.74 \\
\hline 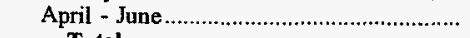 & 523.1 & 11,996 & .61 & 6.18 & 153.2 & 36.75 \\
\hline Total & $1,203.9$ & 12,026 & .62 & 6.42 & 157.5 & $\mathbf{3 7 . 8 8}$ \\
\hline \multicolumn{7}{|l|}{ Indonesia } \\
\hline 1992 & 13.1 & 9,587 & .14 & 1.20 & 166.9 & 32.00 \\
\hline 1993 & 115.8 & 10,620 & .22 & 2.07 & 166.1 & 35.29 \\
\hline 1994 & 437.3 & 10,499 & .22 & 1.82 & 157.4 & 33.06 \\
\hline 1995 & 428.6 & 10,181 & .35 & 1.79 & 149.2 & 30.37 \\
\hline 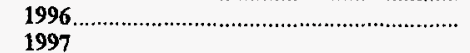 & \multicolumn{5}{|c|}{1997} & 29.26 \\
\hline 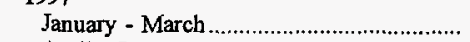 & 218.8 & 9,482 & .37 & 1.67 & 161.3 & 30.59 \\
\hline April - June & 68.2 & 9,521 & .32 & 1.50 & 163.3 & 31.10 \\
\hline 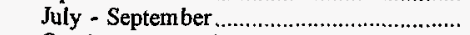 & 219.9 & 9,736 & .35 & 1.20 & 163.3 & 31.80 \\
\hline October - December & 275.0 & 10,042 & .36 & 1.89 & 168.3 & 33.80 \\
\hline 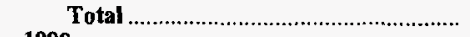 & 782.0 & 9,754 & .36 & 1.60 & 164.6 & 32.10 \\
\hline \multicolumn{7}{|l|}{1998} \\
\hline 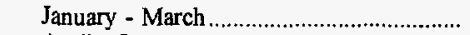 & 73.8 & 9,507 & .38 & 1.10 & 163.3 & 31.05 \\
\hline April - June & 223.5 & 9,623 & .21 & 1.13 & 157.9 & 30.38 \\
\hline Total & 297.3 & 9,594 & .25 & 1.12 & 159.2 & 30.55 \\
\hline
\end{tabular}

See footnotes at the end of Table A6. 
Table A6. Cost and Quality of Imported Coal Received at Electric Utility Plants by Origin, 1992-1998 (Continued)

\begin{tabular}{|c|c|c|c|c|c|c|}
\hline \multirow[b]{2}{*}{ Exporting Country and Time Period } & \multirow{2}{*}{$\begin{array}{l}\text { Quantity } \\
\text { (thousand } \\
\text { short tons) }\end{array}$} & \multicolumn{3}{|c|}{ Average Quality 1} & \multicolumn{2}{|c|}{ Average Cost Delivered } \\
\hline & & $\begin{array}{c}\text { Btu } \\
\text { per Pound }\end{array}$ & $\begin{array}{c}\text { Sulfur } \\
\text { Percent } \\
\text { by Weight }\end{array}$ & $\begin{array}{c}\text { Ash } \\
\text { Percent } \\
\text { by Weight }\end{array}$ & $\begin{array}{l}\text { Cents per } \\
\text { Mitlion Btu }\end{array}$ & $\begin{array}{l}\text { Dollars per } \\
\text { Short Ton }\end{array}$ \\
\hline & \multicolumn{6}{|c|}{ Total of U.S. Electric Utility Plants } \\
\hline \multicolumn{7}{|l|}{ South Africa } \\
\hline 1994 & 127.3 & 11,318 & 0.65 & 12.60 & 181.1 & 41.00 \\
\hline \multicolumn{7}{|l|}{ Venezuela } \\
\hline 1992 & 240.6 & 13,206 & .69 & 7.18 & 164.6 & 43.49 \\
\hline 1993 & 897.5 & 12,874 & .67 & 6.96 & 166.4 & 42.84 \\
\hline 1994 & $1,355.2$ & 12,649 & .76 & 6.61 & 172.3 & 43.60 \\
\hline 1995 & $1,905.7$ & 12,610 & .79 & 6.57 & 194.1 & 48.95 \\
\hline 1996 & $1,767.6$ & 12,686 & .77 & 6.14 & 171.7 & 43.58 \\
\hline \multicolumn{7}{|l|}{1997} \\
\hline 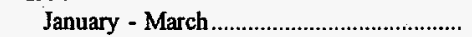 & $164: 2$ & 12,530 & .93 & 7.41 & 161.8 & 40.55 \\
\hline 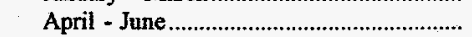 & 331.7 & 12,851 & .84 & 6.16 & 161.7 & 41.56 \\
\hline July - September & 478.9 & 12,616 & .80 & 6.41 & 159.3 & 40.21 \\
\hline 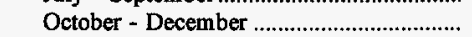 & 360.2 & 12,830 & .82 & 6.12 & 153.7 & 39.43 \\
\hline Total & $1,335.1$ & 12,722 & .83 & 6.40 & 158.7 & 40.38 \\
\hline \multicolumn{7}{|l|}{1998} \\
\hline 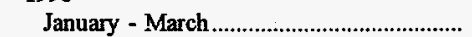 & 611.0 & 13,112 & .66 & 6.29 & 158.6 & 41.59 \\
\hline April - June & 459.7 & 12,849 & .83 & 6.75 & 154.2 & 39.63 \\
\hline Total & $1,070.7$ & 12,999 & .74 & 6.49 & 156.8 & 40.75 \\
\hline
\end{tabular}

1 Data reported on quality of coal as received.

Note: Total may not equal sum of components because of independent rounding.

Source: Federal Energy Regulatory Commission, FERC Form 423, "Monthly Report of Cost and Quality of Fuels for Electric Plants." 
Table A7. Cost and Quality of All Coal Received at Electric Utility Plants that Import Coal by Origin, 1992-1998

\begin{tabular}{|c|c|c|c|c|c|c|}
\hline \multirow[b]{2}{*}{$\begin{array}{l}\text { Time Period and State } \\
\text { or Country of Origin }\end{array}$} & \multirow[b]{2}{*}{$\begin{array}{l}\text { Quantity } \\
\text { (thousand } \\
\text { short tons) }\end{array}$} & \multicolumn{3}{|c|}{ Average Quality 1} & \multicolumn{2}{|c|}{ Average Cost Delivered } \\
\hline & & $\begin{array}{c}\text { Btu } \\
\text { per Pound }\end{array}$ & $\begin{array}{c}\text { Sulfur } \\
\text { Percent } \\
\text { by Weight }\end{array}$ & $\begin{array}{c}\text { Ash } \\
\text { Percent } \\
\text { by Weight }\end{array}$ & $\begin{array}{l}\text { Cents per } \\
\text { Million Btu }\end{array}$ & $\begin{array}{l}\text { Dollars per } \\
\text { Short Ton }\end{array}$ \\
\hline & \multicolumn{6}{|c|}{ Company and Plant: Baltimore Gas and Electric, Brandon Shores } \\
\hline \multicolumn{7}{|l|}{1992} \\
\hline 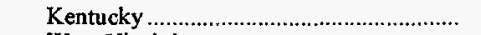 & 215.0 & 12,922 & 0.73 & 7.38 & 154.9 & 40.04 \\
\hline 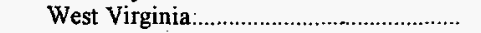 & $2,318.0$ & 12,692 & .68 & 9.92 & 153.4 & 38.93 \\
\hline 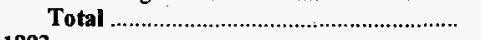 & $2,533.0$ & 12,711 & .68 & 9.70 & 153.5 & 39.03 \\
\hline \multicolumn{7}{|l|}{1993} \\
\hline Kentucky .... & 841.0 & 12,940 & .70 & 7.64 & 158.0 & 40.89 \\
\hline 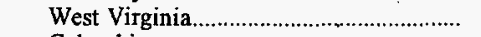 & $1,583.0$ & 12,700 & .67 & 9.65 & 154.4 & 39.21 \\
\hline 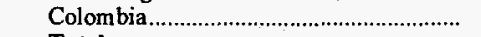 & 224.0 & 12,354 & .64 & 6.32 & 149.8 & 37.02 \\
\hline Total & $2,648.0$ & 12,747 & .68 & 8.73 & 155.2 & 39.56 \\
\hline \multicolumn{7}{|l|}{1994} \\
\hline Kentucky & 664.0 & 12,992 & .72 & 7.72 & 156.5 & 40.66 \\
\hline 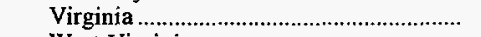 & 1.0 & 12,354 & .74 & 9.30 & 147.2 & 36.37 \\
\hline West Virginia............................................... & $2,728.0$ & 12,496 & .67 & 10.90 & 148.9 & 37.21 \\
\hline Colombia & 88.0 & 12,379 & .66 & 7.36 & 147.3 & 36.46 \\
\hline Total & $3,481.0$ & 12,587 & .68 & 10.20 & 150.3 & 37.85 \\
\hline \multicolumn{7}{|l|}{1995} \\
\hline 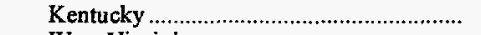 & 667.0 & 13,241 & .73 & 6.41 & 152.5 & 40.39 \\
\hline West Virginia & $2,787.0$ & 12,457 & .68 & 11.05 & 146.2 & 36.42 \\
\hline 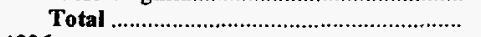 & $3,454.0$ & 12,608 & .69 & 10.15 & 147.5 & 37.19 \\
\hline \multicolumn{7}{|l|}{1996} \\
\hline 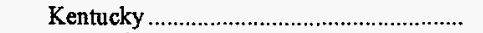 & 611.0 & 13,080 & .74 & 7.09 & 150.9 & 39.48 \\
\hline 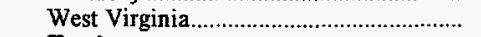 & $3,254.0$ & 12,437 & .68 & 11.34 & 142.2 & 35.37 \\
\hline Total & $3,865.0$ & 12,539 & .69 & 10.66 & 143.6 & 36.02 \\
\hline \multicolumn{7}{|l|}{1997} \\
\hline 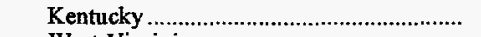 & 485.0 & 13,051 & .75 & 6.84 & 147.3 & 38.45 \\
\hline West Virginia & $2,871.0$ & 12,512 & .67 & 11.37 & 140.9 & 35.27 \\
\hline 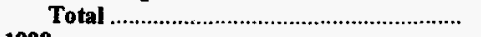 & $\mathbf{3 , 3 5 6 . 0}$ & 12,590 & .68 & 10.71 & 141.9 & 35.73 \\
\hline \multirow{2}{*}{\multicolumn{7}{|c|}{1998 January - March }} \\
\hline & & & & & & \\
\hline 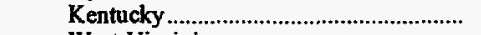 & 115.0 & 12,963 & .76 & 7.26 & 146.0 & 37.86 \\
\hline West Virginia ..................................... & 694.0 & 12,461 & .69 & 11.52 & 138.3 & 34.46 \\
\hline 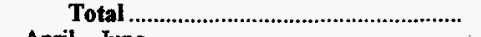 & 809.0 & 12,532 & .70 & 10.92 & 139.4 & 34.94 \\
\hline \multicolumn{7}{|l|}{ April - June } \\
\hline 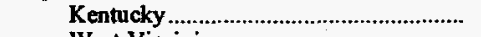 & 144.0 & 13,142 & .75 & 6.12 & 146.4 & 38.49 \\
\hline 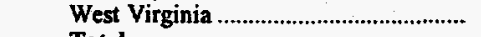 & 701.0 & 12,488 & .69 & 11.47 & 138.6 & 34.62 \\
\hline Total ................................................... & 845.0 & 12,600 & .70 & 10.55 & 140.0 & 35.28 \\
\hline 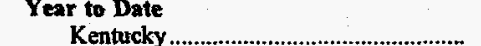 & & & & & & \\
\hline 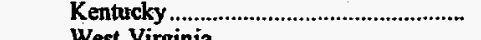 & 259.0 & 13,063 & .75 & 6.62 & 146.3 & 38.21 \\
\hline 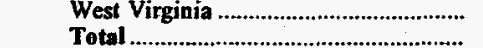 & $1,395.0$ & 12,475 & .69 & 11.49 & 138.4 & 34.54 \\
\hline Total & $1,654.0$ & 12,567 & .70 & 10.73 & 139.7 & 35.11 \\
\hline & & Company ar & It: Cajun Ele & wer Coop, $\mathrm{E}$ & un No. 2 & \\
\hline 1992 & & & & & & \\
\hline Wyoming & $5,343.7$ & 8,368 & 0.46 & 5.30 & 147.5 & 24.69 \\
\hline 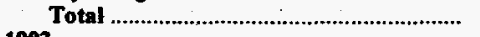 & $\mathbf{5 , 3 4 3 . 7}$ & 8,368 & .46 & 5.30 & 147.5 & 24.69 \\
\hline 1993 & & & & & & \\
\hline Wyoming & $5,701.1$ & 8,332 & .43 & 5.27 & 151.9 & 25.31 \\
\hline Total & $5,701.1$ & 8,332 & .43 & 5.27 & 151.9 & 25.31 \\
\hline 1994 & & & & & & \\
\hline Colorado & 37.4 & 11,957 & .45 & 8.01 & 156.4 & 37.40 \\
\hline Wyoming & $5,588.0$ & 8,442 & .36 & 4.93 & 152.2 & 25.70 \\
\hline 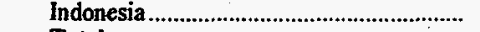 & 169.2 & 9,702 & .10 & 1.20 & 166.8 & 32.36 \\
\hline 1998 & $5,794.6$ & 8,502 & .35 & 4.84 & 152.8 & 25.97 \\
\hline Wyoming & $5,844.5$ & 8,469 & .35 & 4.99 & 157.6 & 26.69 \\
\hline 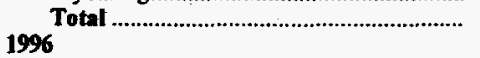 & $5,844.5$ & 8,469 & .35 & 4.99 & 157.6 & 26.69 \\
\hline 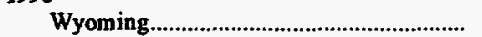 & $5,394.2$ & 8,500 & .41 & 5.18 & 161.1 & 27.38 \\
\hline Total & $5,394.2$ & $\mathbf{8 , 5 0 0}$ & .41 & 5.18 & 161.1 & 27.38 \\
\hline 1997 & & & & & & \\
\hline Wyoming & $5,762.0$ & 8,467 & .45 & 5.27 & 154.9 & 26.23 \\
\hline 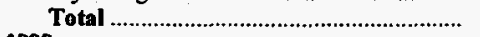 & $5,762.0$ & 8,467 & .45 & 5.27 & 154.9 & 26.23 \\
\hline 1998 & & & & & & \\
\hline January - March & & & & & & \\
\hline 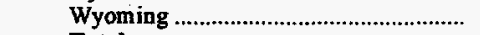 & $1,545.8$ & 8,457 & .44 & 5.34 & 145.2 & 24.56 \\
\hline Total & $1,545.8$ & 8,457 & .44 & 5.34 & 145.2 & 24.56 \\
\hline
\end{tabular}

See footnotes at the end of Table A7. 
Table A7. Cost and Quality of All Coal Received at Electric Utility Plants that Import Coal by Origin, 1992-1998 (Continued)

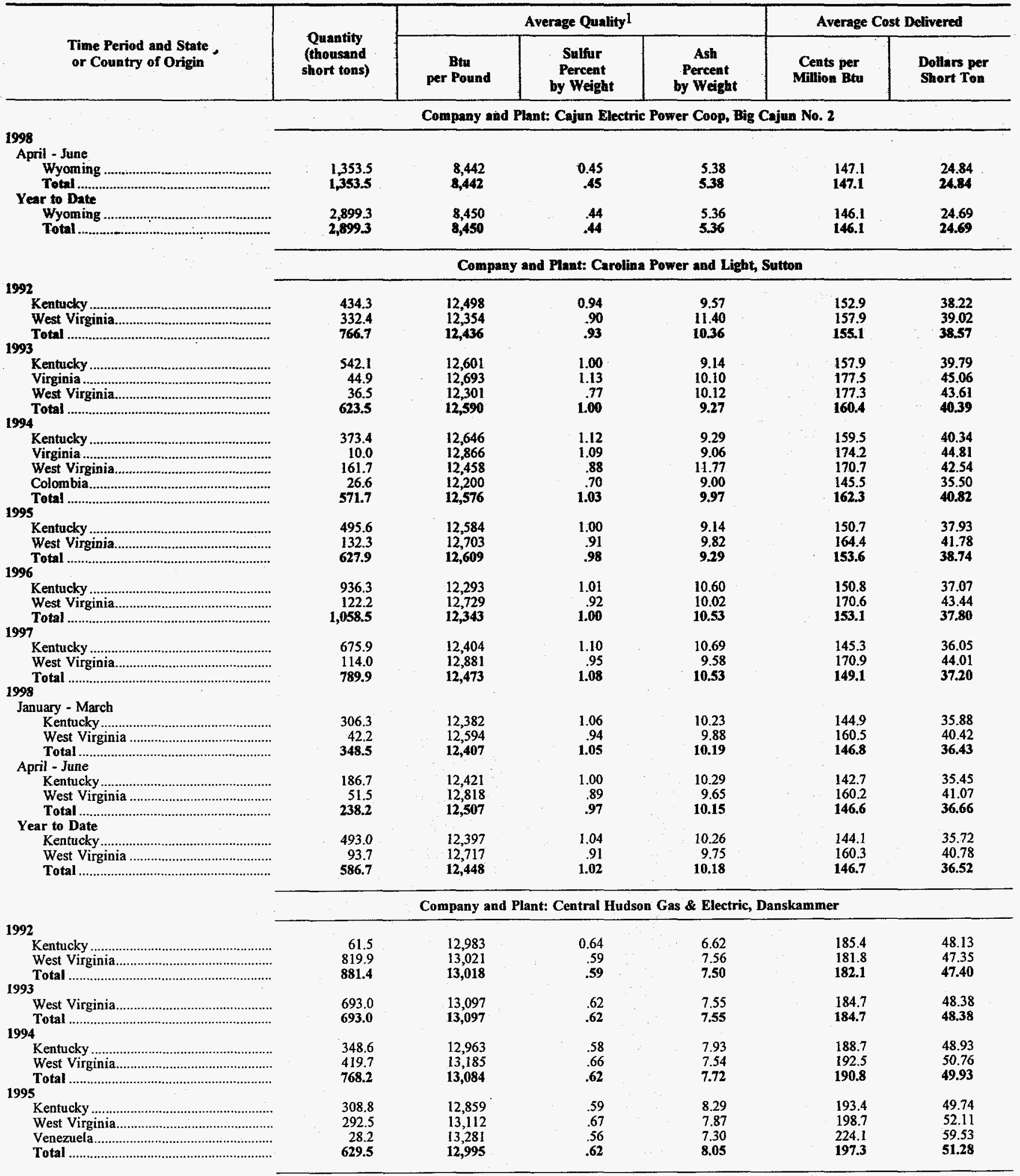

See footnotes at the end of Table A7. 
Table A7. Cost and Quality of All Coal Received at Electric Utility Plants that Import Coal by Origin, 1992-1998 (Continued)

\begin{tabular}{|c|c|c|c|c|c|c|}
\hline \multirow[b]{2}{*}{$\begin{array}{l}\text { Time Period and State } \\
\text { or Country of Origin }\end{array}$} & \multirow[b]{2}{*}{$\begin{array}{l}\text { Quantity } \\
\text { (thousand } \\
\text { short tons) }\end{array}$} & \multicolumn{3}{|c|}{ Average Quality 1} & \multicolumn{2}{|c|}{ Average Cost Delivered } \\
\hline & & $\begin{array}{c}\text { Btu } \\
\text { per Pound }\end{array}$ & $\begin{array}{c}\text { Sulfur } \\
\text { Percent } \\
\text { by Weight }\end{array}$ & $\begin{array}{c}\text { Ash } \\
\text { Percent } \\
\text { by Weight }\end{array}$ & $\begin{array}{l}\text { Cents per } \\
\text { Million Btu }\end{array}$ & $\begin{array}{l}\text { Dollars per } \\
\text { Short Ton }\end{array}$ \\
\hline & \multicolumn{6}{|c|}{ Company and Plant: Central Hudson Gas \& Electric, Danskammer } \\
\hline \multicolumn{7}{|l|}{1996} \\
\hline 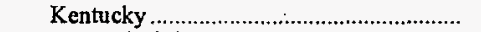 & 462.4 & 12,822 & 0.65 & 8.55 & 193.2 & 49.55 \\
\hline 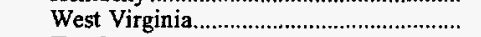 & 351.4 & 13,061 & .68 & 7.86 & 200.5 & 52.38 \\
\hline Total & 813.8 & 12,925 & .66 & 8.25 & 196.4 & 50.77 \\
\hline \multicolumn{7}{|l|}{1997} \\
\hline Kentucky & 72.3 & 12,933 & .66 & 8.45 & 180.2 & 46.62 \\
\hline 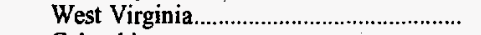 & 246.2 & 13,191 & .62 & 7.78 & 177.0 & 46.70 \\
\hline Colombia & 147.0 & 13,032 & .65 & 7.17 & 171.3 & 44.64 \\
\hline Venezuela & 350.4 & 13,172 & $65^{\circ}$ & 6.40 & 173.1 & 45.61 \\
\hline Total & 815.9 & 13,131 & .64 & 7.14 & 174.6 & 45.85 \\
\hline \multicolumn{7}{|l|}{$\begin{array}{l}1998 \\
\text { January - March }\end{array}$} \\
\hline 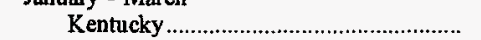 & 77.5 & 12,955 & .69 & 8.38 & 174.1 & 45.10 \\
\hline 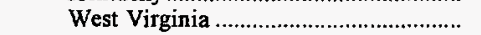 & 9.7 & 12,979 & .65 & 8.50 & 173.3 & 44.99 \\
\hline 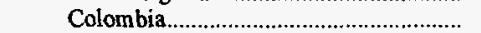 & 34.8 & 13,309 & .62 & 7.38 & 169.8 & 45.20 \\
\hline 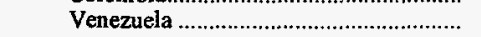 & 122.6 & 13,311 & .59 & 7.02 & 169.8 & 45.21 \\
\hline Total & 244.6 & 13,185 & .63 & 7.56 & 171.3 & 45.16 \\
\hline \multicolumn{7}{|l|}{ April - June } \\
\hline 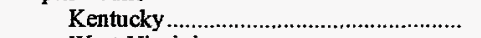 & 116.2 & 12,971 & .65 & 8.42 & 170.8 & 44.30 \\
\hline 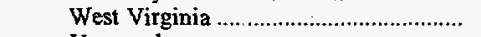 & 10.2 & 12,704 & .69 & 10.95 & 166.6 & 42.33 \\
\hline 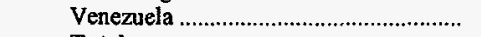 & 104.9 & 13,184 & .65 & 7.70 & 170.0 & 44.82 \\
\hline Total & 231.3 & 13,055 & .65 & 8.21 & 170.2 & 44.45 \\
\hline \multicolumn{7}{|l|}{ Year to Date } \\
\hline 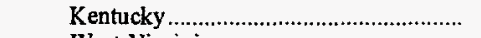 & 193.7 & 12,964 & .67 & 8.40 & 172.1 & 44.62 \\
\hline 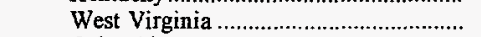 & 19.9 & 12,838 & .67 & 9.76 & 169.9 & 43.62 \\
\hline 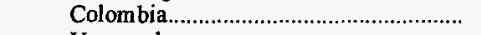 & 34.8 & 13,309 & .62 & 7.38 & 169.8 & 45.20 \\
\hline Venezuela & 227.5 & 13,252 & .62 & 7.33 & 169.9 & 45.03 \\
\hline \multirow[t]{2}{*}{ Total } & 475.9 & 13,122 & .64 & 7.87 & 170.8 & 44.82 \\
\hline & \multicolumn{6}{|c|}{ Company and Plant: City Public Service, San Antonio JT Deely/Spruce } \\
\hline 1992 & & & & & & \\
\hline 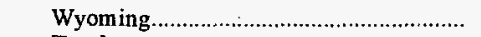 & $3,542,9$ & 8,489 & 0.33 & 5.43 & 127.9 & 21.72 \\
\hline 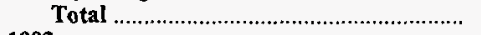 & $3,542.9$ & 8,489 & .33 & 5.43 & 127.9 & 21.72 \\
\hline 1993 & & & & & & \\
\hline 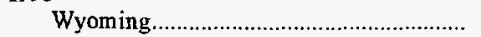 & $5,286.0$ & 8,423 & .33 & 5.22 & 118.2 & 19.92 \\
\hline Total & $5,286.0$ & 8,423 & .33 & 5.22 & 118.2 & 19.92 \\
\hline 1994 & & & & & & \\
\hline Wyoming & $4,606.0$ & 8,406 & .34 & 5.42 & 112.9 & 18.98 \\
\hline 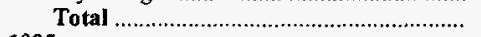 & $4,606.0$ & 8,406 & .34 & 5.42 & 112.9 & 18.98 \\
\hline 1995 & & & & & & \\
\hline Colorado & 22.0 & 10,536 & .41 & 5.20 & 129.5 & 27.29 \\
\hline 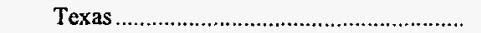 & 40.0 & 10,540 & 1.03 & 21.69 & 120.2 & 25.33 \\
\hline 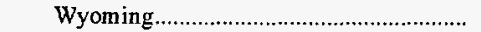 & $5,284.0$ & 8,393 & .36 & 5.71 & 111.1 & 18.66 \\
\hline 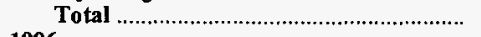 & $5,346.0$ & 8,418 & .36 & 5.83 & 111.3 & 18.74 \\
\hline 1996 & & & & & & \\
\hline Colorado & 11.0 & 10,751 & .59 & 5.10 & 131.2 & 28.21 \\
\hline 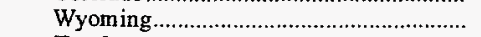 & $5,488.0$ & 8,333 & .35 & 6.11 & 101.8 & 16.96 \\
\hline 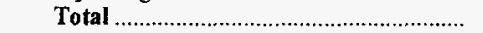 & $5,499.0$ & $\mathbf{8 , 3 3 8}$ & .35 & 6.11 & 101.9 & 16.99 \\
\hline 1997 & & & & & & \\
\hline Wyoming & $4,781.0$ & 8,350 & .36 & 6.00 & 97.2 & 16.23 \\
\hline 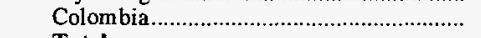 & 73.0 & 11,603 & .34 & 3.89 & 176.9 & 41.06 \\
\hline 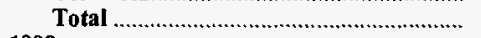 & $4,854.0$ & 8,399 & .36 & 5.96 & 98.9 & 16.61 \\
\hline 1998 & & & & & & \\
\hline January - March & & & & & & \\
\hline Wyoming & $1,160.0$ & 8,348 & .35 & 5.98 & 98.2 & 16.40 \\
\hline 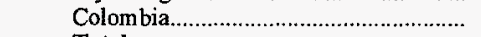 & 24.0 & 11,600 & .33 & 3.80 & 200.6 & 46.54 \\
\hline Total & $1,184.0$ & 8,414 & .35 & 5.93 & 101.1 & 17.01 \\
\hline April - June & & & & & & \\
\hline Wyoming & $1,290.0$ & 8,361 & .35 & 6.12 & 99.7 & 16.67 \\
\hline Venezuela & 9.0 & 12,855 & .70 & 6.00 & 185.8 & 47.77 \\
\hline Total & $1,299.0$ & 8,392 & .35 & 6.12 & 100.6 & 16.88 \\
\hline Year to Date & & & & & & \\
\hline 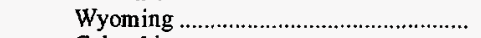 & $2,450.0$ & 8,355 & .35 & 6.05 & 99.0 & 16.54 \\
\hline Colombia & 24.0 & 11,600 & .33 & 3.80 & 200.6 & 46.54 \\
\hline 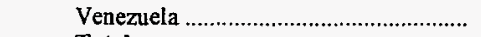 & 9.0 & 12,855 & .70 & 6.00 & 185.8 & 47.77 \\
\hline Total & $2,483.0$ & 8,403 & .35 & 6.03 & 100.8 & 16.94 \\
\hline
\end{tabular}

See footnotes at the end of Table A7. 
Table A7. Cost and Quality of All Coal Received at Electric Utility Plants that Import Coal by Origin, 1992-1998 (Continued)

\begin{tabular}{|c|c|c|c|c|c|c|}
\hline \multirow[b]{2}{*}{$\begin{array}{l}\text { Time Périod and State } \\
\text { or Country of Origin }\end{array}$} & \multirow[b]{2}{*}{$\begin{array}{l}\text { Quantity } \\
\text { (thousand } \\
\text { short tons) }\end{array}$} & \multicolumn{3}{|c|}{ Average Quality 1} & \multicolumn{2}{|c|}{ Average Cost Delivered } \\
\hline & & $\begin{array}{c}\text { Btu } \\
\text { per Pound }\end{array}$ & $\begin{array}{c}\text { Sulfur } \\
\text { Percent } \\
\text { by Weight }\end{array}$ & $\begin{array}{c}\text { Ash } \\
\text { Percent } \\
\text { by Weight }\end{array}$ & $\begin{array}{l}\text { Cents per } \\
\text { Million Btu }\end{array}$ & $\begin{array}{l}\text { Dollars per } \\
\text { Short Ton }\end{array}$ \\
\hline & \multicolumn{6}{|c|}{ Company and Plant: Central Power and Light (CSW), Coleto Creek } \\
\hline 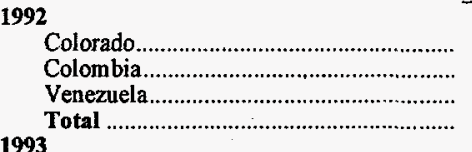 & $\begin{array}{r}1,780.7 \\
37.2 \\
42.5 \\
1,860.4\end{array}$ & $\begin{array}{l}10,885 \\
12,892 \\
13,214 \\
10,978\end{array}$ & $\begin{array}{r}0.39 \\
.62 \\
.66 \\
.40\end{array}$ & $\begin{array}{l}6.32 \\
7.90 \\
7.20 \\
6.37\end{array}$ & $\begin{array}{l}205.0 \\
174.5 \\
175.8 \\
203.5\end{array}$ & $\begin{array}{l}44.63 \\
44.99 \\
46.46 \\
44.68\end{array}$ \\
\hline 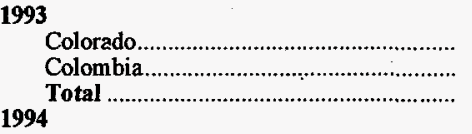 & $\begin{array}{r}1,778.0 \\
122.5 \\
1,900.5\end{array}$ & $\begin{array}{l}10,577 \\
12,109 \\
10,676\end{array}$ & $\begin{array}{l}.40 \\
.60 \\
.41\end{array}$ & $\begin{array}{l}6.61 \\
5.90 \\
6.56\end{array}$ & $\begin{array}{l}203.1 \\
148.5 \\
199.1\end{array}$ & $\begin{array}{l}42.96 \\
35.98 \\
42.51\end{array}$ \\
\hline $\begin{array}{l}\text { Colorado. } \\
\text { Colombia } \\
\text { Total }\end{array}$ & $\begin{array}{r}1,664.9 \\
153.4 \\
1,818.3\end{array}$ & $\begin{array}{l}10,760 \\
11,929 \\
10,858\end{array}$ & $\begin{array}{l}.41 \\
.55 \\
.42\end{array}$ & $\begin{array}{l}6.77 \\
5.03 \\
6.63\end{array}$ & $\begin{array}{l}199.7 \\
148.9 \\
195.0\end{array}$ & $\begin{array}{l}42.98 \\
35.51 \\
42.35\end{array}$ \\
\hline 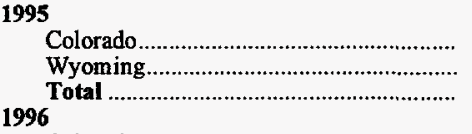 & $\begin{array}{r}1,724.7 \\
119.4 \\
1,844.1\end{array}$ & $\begin{array}{r}11,092 \\
8,764 \\
10,941\end{array}$ & $\begin{array}{l}.42 \\
.34 \\
.41\end{array}$ & $\begin{array}{l}6.92 \\
5.20 \\
6.81\end{array}$ & $\begin{array}{l}169.2 \\
163.5 \\
168.9\end{array}$ & $\begin{array}{l}37.53 \\
28.66 \\
36.95\end{array}$ \\
\hline 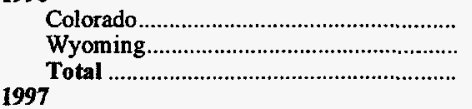 & $\begin{array}{r}1,823.5 \\
188.0 \\
2,011.6\end{array}$ & $\begin{array}{r}10,482 \\
8,492 \\
10,296\end{array}$ & $\begin{array}{l}.39 \\
.31 \\
.38\end{array}$ & $\begin{array}{l}5.77 \\
5.73 \\
5.77\end{array}$ & $\begin{array}{l}133.8 \\
142.9 \\
134.5\end{array}$ & $\begin{array}{l}28.06 \\
24.27 \\
\mathbf{2 7 . 7 0}\end{array}$ \\
\hline 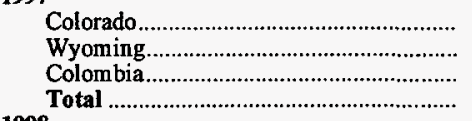 & $\begin{array}{r}1,517.0 \\
252.4 \\
26.0 \\
1,795.4\end{array}$ & $\begin{array}{r}10,281 \\
8,713 \\
11,665 \\
10,081\end{array}$ & $\begin{array}{l}.38 \\
.47 \\
.47 \\
.40\end{array}$ & $\begin{array}{l}6.10 \\
5.12 \\
6.00 \\
5.96\end{array}$ & $\begin{array}{l}135.3 \\
134.4 \\
173.2 \\
135.9\end{array}$ & $\begin{array}{l}27.83 \\
23.41 \\
40.41 \\
27.39\end{array}$ \\
\hline \multicolumn{7}{|l|}{$\begin{array}{l}1998 \\
\text { January - March }\end{array}$} \\
\hline 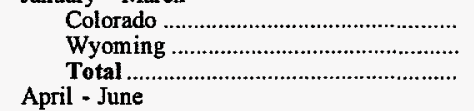 & $\begin{array}{l}376.4 \\
172.5 \\
549.0\end{array}$ & $\begin{array}{r}10,469 \\
8,735 \\
9,924\end{array}$ & $\begin{array}{l}.35 \\
.41 \\
.37\end{array}$ & $\begin{array}{l}5.80 \\
5.22 \\
5.62\end{array}$ & $\begin{array}{l}140.8 \\
131.3 \\
138.2\end{array}$ & $\begin{array}{l}29.49 \\
22.94 \\
27.43\end{array}$ \\
\hline 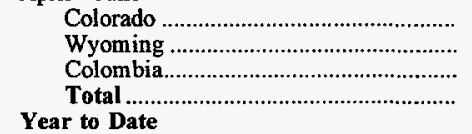 & $\begin{array}{r}248.2 \\
287.8 \\
23.7 \\
\mathbf{5 5 9 . 7}\end{array}$ & $\begin{array}{r}10,472 \\
8,681 \\
12,760 \\
9,648\end{array}$ & $\begin{array}{l}.36 \\
.21 \\
.66 \\
.30\end{array}$ & $\begin{array}{l}5.50 \\
4.43 \\
6.60 \\
4.99\end{array}$ & $\begin{array}{l}139.8 \\
130.3 \\
188.3 \\
138.1\end{array}$ & $\begin{array}{l}29.28 \\
22.63 \\
48.05 \\
26.65\end{array}$ \\
\hline \multirow[t]{2}{*}{ 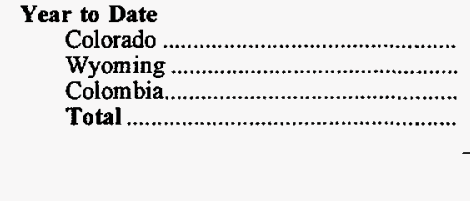 } & $\begin{array}{r}624.6 \\
460.3 \\
23.7 \\
1,108.6\end{array}$ & $\begin{array}{r}10,470 \\
8,701 \\
12,760 \\
9,785\end{array}$ & $\begin{array}{l}.36 \\
.29 \\
.66 \\
.33\end{array}$ & $\begin{array}{l}5.68 \\
4.73 \\
6.60 \\
\mathbf{5 . 3 0}\end{array}$ & $\begin{array}{l}140.4 \\
130.7 \\
188.3 \\
138.2\end{array}$ & $\begin{array}{l}29.40 \\
22.75 \\
48.05 \\
27.04\end{array}$ \\
\hline & \multicolumn{6}{|c|}{ Company and Plant: Delmarva Power \& Light, Edgemoor } \\
\hline 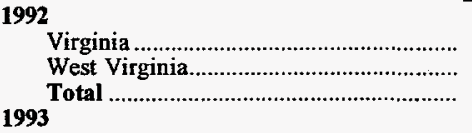 & $\begin{array}{r}90.2 \\
463.8 \\
554.0\end{array}$ & $\begin{array}{l}13,101 \\
13,101 \\
\mathbf{1 3 , 1 0 1}\end{array}$ & $\begin{array}{r}0.82 \\
.79 \\
.80\end{array}$ & $\begin{array}{l}8.68 \\
8.64 \\
8.65\end{array}$ & $\begin{array}{l}201.3 \\
180.0 \\
183.4\end{array}$ & $\begin{array}{l}52.74 \\
47.16 \\
48.06\end{array}$ \\
\hline 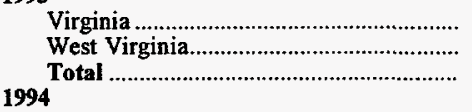 & $\begin{array}{l}192.3 \\
250.2 \\
442.5\end{array}$ & $\begin{array}{l}13,209 \\
13,171 \\
13,188\end{array}$ & $\begin{array}{l}.86 \\
.81 \\
.83\end{array}$ & $\begin{array}{l}8.00 \\
8.63 \\
8.36\end{array}$ & $\begin{array}{l}200.3 \\
178.0 \\
187.7\end{array}$ & $\begin{array}{l}52.90 \\
46.88 \\
49.50\end{array}$ \\
\hline 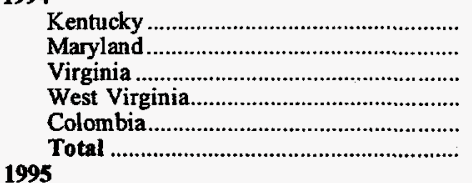 & $\begin{array}{r}7.0 \\
13.3 \\
28.6 \\
604.3 \\
22.0 \\
675.2\end{array}$ & $\begin{array}{l}12,991 \\
13,070 \\
12,995 \\
13,074 \\
12,370 \\
13,046\end{array}$ & $\begin{array}{l}.57 \\
.74 \\
.88 \\
.79 \\
.58 \\
.78\end{array}$ & $\begin{array}{l}6.53 \\
6.23 \\
8.72 \\
8.74 \\
5.98 \\
8.58\end{array}$ & $\begin{array}{l}165.3 \\
168.2 \\
164.7 \\
157.9 \\
168.2 \\
158.8\end{array}$ & $\begin{array}{l}42.95 \\
43.97 \\
42.80 \\
41.29 \\
41.61 \\
41.44\end{array}$ \\
\hline 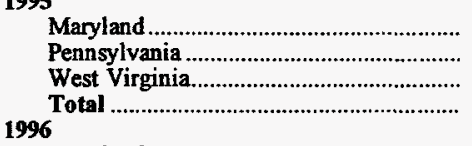 & $\begin{array}{r}37.9 \\
.6 \\
432.6 \\
471.1\end{array}$ & $\begin{array}{l}12,867 \\
12,431 \\
12,988 \\
12,978\end{array}$ & $\begin{array}{l}.76 \\
.82 \\
.78 \\
.78\end{array}$ & $\begin{array}{r}9.73 \\
10.42 \\
9.04 \\
9.10\end{array}$ & $\begin{array}{l}161.6 \\
154.7 \\
162.9 \\
162.8\end{array}$ & $\begin{array}{l}41.59 \\
38.46 \\
42.31 \\
42.25\end{array}$ \\
\hline 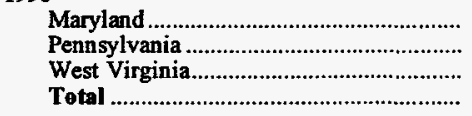 & $\begin{array}{r}14.7 \\
.1 \\
487.3 \\
502.0\end{array}$ & $\begin{array}{l}12,868 \\
12,559 \\
12,887 \\
12,886\end{array}$ & $\begin{array}{l}.69 \\
.82 \\
.77 \\
.76\end{array}$ & $\begin{array}{r}10.30 \\
10.42 \\
9.67 \\
9.69\end{array}$ & $\begin{array}{l}161.0 \\
153.1 \\
160.2 \\
160.2\end{array}$ & $\begin{array}{l}41.43 \\
38.46 \\
41.28 \\
41.29\end{array}$ \\
\hline
\end{tabular}

See footnotes at the end of Table A7. 
Table A7. Cost and Quality of All Coal Received at Electric Utility Plants that Import Coal by Origin, 1992-1998 (Continued)

\begin{tabular}{|c|c|c|c|c|c|c|}
\hline \multirow[b]{2}{*}{$\begin{array}{l}\text { Time Period and State } \\
\text { or Country of Origin }\end{array}$} & \multirow[b]{2}{*}{$\begin{array}{l}\text { Quantity } \\
\text { (thousand } \\
\text { short tons) }\end{array}$} & \multicolumn{3}{|c|}{ Average Quality 1} & \multicolumn{2}{|c|}{ Average Cost Delivered } \\
\hline & & $\begin{array}{c}\text { Btu } \\
\text { per Pound }\end{array}$ & $\begin{array}{c}\text { Sulfur } \\
\text { Percent } \\
\text { by Weight }\end{array}$ & $\begin{array}{c}\text { Ash } \\
\text { Percent } \\
\text { by Weight }\end{array}$ & $\begin{array}{l}\text { Cents per } \\
\text { Million Btu }\end{array}$ & $\begin{array}{l}\text { Dollars per } \\
\text { Short Ton }\end{array}$ \\
\hline & \multicolumn{6}{|c|}{ Company and Plant: Delmarva Power \& Light, Edgemoor } \\
\hline \multicolumn{7}{|l|}{1997} \\
\hline 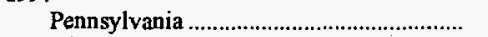 & 18.3 & 10,772 & 0.58 & 11.12 & 151.2 & 32.57 \\
\hline 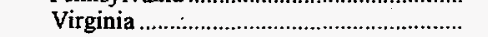 & 187.5 & 13,211 & .85 & 8.98 & 156.7 & 41.40 \\
\hline 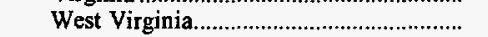 & 299.3 & 12,796 & .76 & 10.25 & 161.5 & 41.34 \\
\hline Total & 505.2 & 12,877 & .79 & 9.81 & 159.4 & 41.05 \\
\hline 1998 & & & & & & . \\
\hline \multicolumn{7}{|l|}{ January - March } \\
\hline 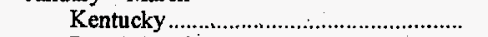 & 10.7 & 12,935 & .79 & 6.40 & 163.8 & 42.38 \\
\hline 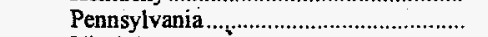 & 3.4 & 10,573 & .66 & 9.97 & 150.3 & 31.79 \\
\hline 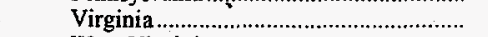 & 8.6 & 13,159 & .83 & 8.30 & 158.1 & 41.61 \\
\hline 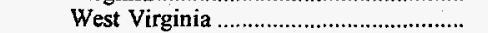 & 115.5 & 12,648 & .73 & 10.38 & 160.5 & 40.60 \\
\hline Total & 138.1 & 12,651 & .74 & 9.93 & 160.4 & 40.58 \\
\hline \multicolumn{7}{|l|}{ April - June } \\
\hline Pennsylvania & 2.0 & 10,798 & .58 & 10.00 & 151.3 & 32.67 \\
\hline 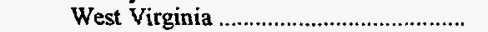 & 91.3 & 12,663 & .70 & 10.85 & 159.6 & 40.42 \\
\hline 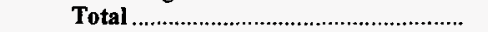 & 93.3 & 12,623 & .69 & 10.84 & 159.4 & 40.25 \\
\hline \multicolumn{7}{|l|}{ Year to Date } \\
\hline 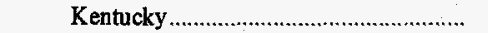 & 10.7 & 12,935 & .79 & 6.40 & 163.8 & 42.38 \\
\hline 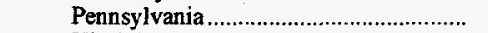 & 5.4 & 10,657 & .63 & 9.98 & 150.7 & 32.12 \\
\hline Virginia & 8.6 & 13,159 & .83 & 8.30 & 158.1 & 41.61 \\
\hline 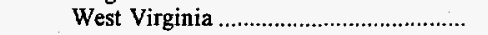 & 206.7 & 12,655 & .72 & 10.59 & 160.1 & 40.52 \\
\hline \multirow[t]{2}{*}{ 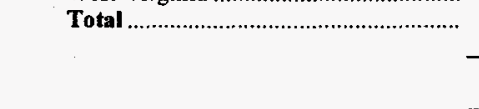 } & 231.4 & 12,640 & .72 & 10.30 & 160.0 & 40.45 \\
\hline & \multicolumn{6}{|c|}{ Company and Plant: Delmarva Power and Light, Indian River } \\
\hline \multicolumn{7}{|l|}{1992} \\
\hline 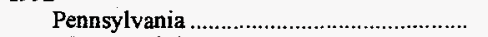 & 137.4 & 13,104 & 1.40 & 9.31 & 177.9 & 46.62 \\
\hline 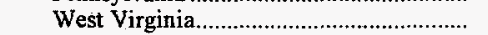 & 840.2 & 13,034 & 1.12 & 8.88 & 166.1 & 43.29 \\
\hline Total & 977.6 & 13,044 & 1.16 & 8.94 & 167.7 & 43.76 \\
\hline 1993 & & & & & & \\
\hline 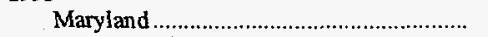 & 45.1 & 12,966 & 1.29 & 9.49 & 160.4 & 41.59 \\
\hline 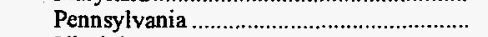 & 216.3 & 12,971 & 1.32 & 9.58 & 164.2 & 42.60 \\
\hline Virginia & 14.0 & 13,273 & $.7 \overline{7}$ & 6.90 & 188.2 & 49.96 \\
\hline 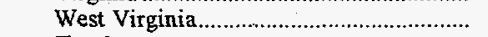 & $1,290.6$ & 12,980 & .90 & 9.25 & 163.3 & 42.40 \\
\hline Total & $1,565.9$ & 12,981 & .97 & 9.28 & 163.6 & 42.48 \\
\hline 1994 & & & & & & \\
\hline 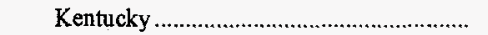 & 29.4 & 12,899 & .59 & 6.90 & 179.3 & 46.25 \\
\hline 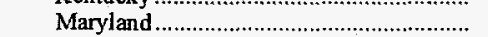 & 125.0 & 13,164 & 1.44 & 10.23 & 147.9 & 38.95 \\
\hline 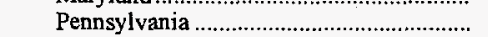 & 251.2 & 13,004 & 1.29 & 8.96 & 161.1 & 41.89 \\
\hline 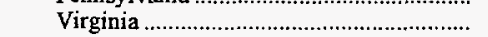 & 56.5 & 13,125 & .76 & 7.30 & 180.8 & 47.45 \\
\hline West Virginia & $1,146.2$ & 12,858 & .88 & 9.45 & 164.4 & 42.27 \\
\hline Total & $1,608.3$ & 12,915 & .98 & 9.31 & 163.4 & 42.21 \\
\hline 1995 & & & & & & \\
\hline Maryland & 227.9 & 13,155 & 1.38 & 9.89 & 149.3 & 39.29 \\
\hline 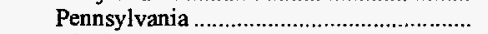 & 352.3 & 13,227 & 1.44 & 6.90 & 148.8 & 39.37 \\
\hline 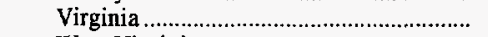 & 23.2 & 13,382 & 1.46 & 6.53 & 143.0 & 38.28 \\
\hline 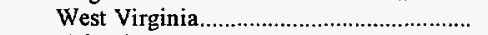 & 638.7 & 13,050 & .76 & 8.71 & 172.6 & 45.04 \\
\hline Colombia & 7.1 & 13,141 & .75 & 7.07 & 180.3 & 47.39 \\
\hline Total & $1,249.2$ & 13,126 & 1.08 & 8.36 & 161.1 & 42.28 \\
\hline 1996 & & & & & & \\
\hline 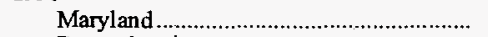 & 262.5 & 13,158 & 1.41 & 9.26 & 149.7 & 39.38 \\
\hline 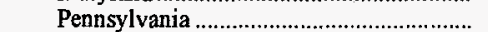 & 390.6 & 13,271 & 1.44 & 6.73 & 146.3 & 38.84 \\
\hline 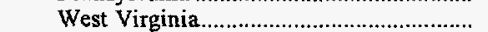 & 589.8 & 12,907 & .76 & 8.99 & 172.1 & 44.42 \\
\hline 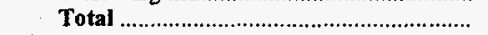 & $1,242.9$ & 13,074 & 1.11 & 8.33 & 159.1 & 41.60 \\
\hline 1997 & & & & & & \\
\hline 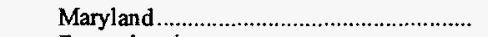 & 160.8 & 13,158 & 1.46 & 9.17 & 147.8 & 38.89 \\
\hline 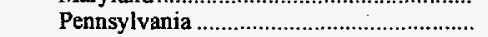 & 482.5 & 13,275 & 1.39 & 7.22 & 141.3 & 37.52 \\
\hline 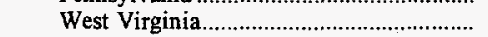 & 533.9 & 13,017 & .70 & 8.70 & 172.5 & 44.90 \\
\hline Total & $1,177.1$ & 13,142 & 1.08 & 8.16 & 156.2 & 41.05 \\
\hline 1998 & & & & & & \\
\hline January - March & & & & & & \\
\hline 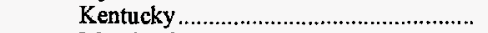 & 10.5 & 13,058 & .58 & 6.50 & 181.5 & 47.40 \\
\hline 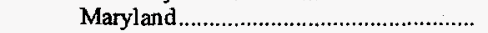 & 7.1 & 13,367 & 1.56 & 9.00 & 146.1 & 39.06 \\
\hline Pennsylvania & 180.4 & 13,180 & 1.39 & 7.29 & 142.6 & 37.58 \\
\hline 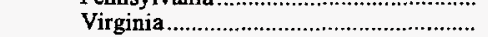 & 19.3 & 13,304 & .80 & 7.68 & 178.0 & 47.35 \\
\hline West Virginia & 46.3 & 12,615 & .67 & 10.48 & 172.4 & 43.49 \\
\hline Total & 263.5 & 13,090 & 1.19 & 7.90 & 151.9 & 39.76 \\
\hline
\end{tabular}

See footnotes at the end of Table A7. 
Table A7. Cost and Quality of All Coal Received at Electric Utility Plants that Import Coal by Origin, 1992-1998 (Continued)

\begin{tabular}{|c|c|c|c|c|c|c|}
\hline \multirow[b]{2}{*}{$\begin{array}{l}\text { Time Period and State } \\
\text { or Country of Origin }\end{array}$} & \multirow[b]{2}{*}{$\begin{array}{l}\text { Quantity } \\
\text { (thousand } \\
\text { short tons) }\end{array}$} & \multicolumn{3}{|c|}{ Average Quality 1} & \multicolumn{2}{|c|}{ Average Cost Delivered } \\
\hline & & $\begin{array}{c}\text { Btu } \\
\text { per Pound }\end{array}$ & $\begin{array}{c}\text { Sulfur } \\
\text { Percent } \\
\text { by Weight }\end{array}$ & $\begin{array}{c}\text { Ash } \\
\text { Percent } \\
\text { by Weight }\end{array}$ & $\begin{array}{l}\text { Cents per } \\
\text { Million Btu }\end{array}$ & $\begin{array}{l}\text { Dollars per } \\
\text { Short Ton }\end{array}$ \\
\hline & \multicolumn{6}{|c|}{ Company and Plant: Delmarva Power and Light, Indian River } \\
\hline \multicolumn{7}{|l|}{1998} \\
\hline \multicolumn{7}{|l|}{ April - June } \\
\hline 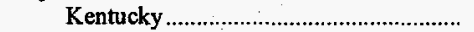 & 32.8 & 12,684 & 0.62 & 6.40 & 178.1 & 45.17 \\
\hline 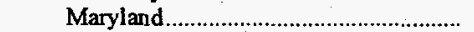 & 49.5 & 13,028 & 1.46 & 8.56 & 146.3 & 38.13 \\
\hline Pennsylyania ............................................ & 54.0 & 13,155 & 1.36 & 6.79 & 142.2 & 37.40 \\
\hline 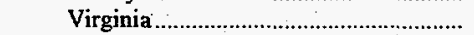 & 37.4 & 13,700 & .84 & 5.84 & 177.9 & 48.75 \\
\hline 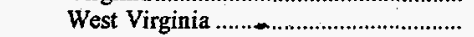 & 61.6 & 12,436 & .66 & 11.90 & 165.8 & 41.23 \\
\hline Total & 235.2 & 12,961 & 1.01 & 8.29 & 159.9 & 41.44 \\
\hline \multicolumn{7}{|l|}{ Year to Date } \\
\hline Kentucky & 43.2 & 12,775 & .61 & 6.42 & 178.9 & 45.71 \\
\hline Maryland & 56.7 & 13,070 & 1.47 & 8.61 & 146.3 & 38.25 \\
\hline 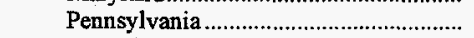 & 234.3 & 13,175 & 1.39 & 7.18 & 142.5 & 37.54 \\
\hline Virginia & 56.6 & 13,565 & .82 & 6.47 & 177.9 & 48.28 \\
\hline West Virginia & 107.8 & 12,513 & .67 & 11.30 & 168.6 & 42.20 \\
\hline \multirow[t]{2}{*}{ Total } & 498.7 & 13,029 & 1.11 & 8.08 & 155.6 & 40.56 \\
\hline & \multicolumn{6}{|c|}{ Company and Plant: Detroit Edison Co, River Rouge } \\
\hline \multicolumn{7}{|l|}{1992} \\
\hline 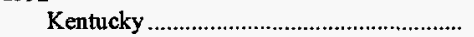 & 62.0 & 12,795 & 0.80 & 8.07 & 194.5 & 49.77 \\
\hline 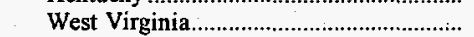 & 697.0 & 12,570 & .68 & 11.04 & 156.1 & 39.24 \\
\hline Wyoming & 209.0 & 8,720 & .24 & 4.80 & 105.3 & 18.37 \\
\hline Total & 968.0 & 11,753 & .59 & 9.50 & 150.6 & 35.41 \\
\hline \multicolumn{7}{|l|}{1993} \\
\hline 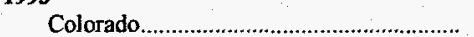 & 11.0 & 11,620 & .53 & 8.80 & 147.6 & 34.30 \\
\hline Kentucky & 359.0 & 12,638 & .87 & 8.49 & 175.7 & 44.42 \\
\hline Virginia & 10.0 & 13,583 & .81 & 5.40 & 200.3 & 54.41 \\
\hline 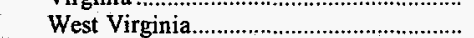 & 479.0 & 12,457 & .72 & 11.64 & 155.2 & 38.67 \\
\hline 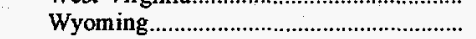 & 399.0 & 8,752 & .25 & 4.91 & 104.0 & 18.21 \\
\hline 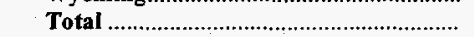 & $1,258.0$ & 11,335 & .61 & 8.53 & 149.6 & 33.91 \\
\hline \multicolumn{7}{|l|}{1994} \\
\hline Colorado & 21.0 & 11,838 & .48 & 8.38 & 146.2 & 34.61 \\
\hline 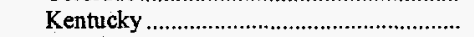 & 246.0 & 12,658 & .81 & 8.22 & 178.4 & 45.17 \\
\hline 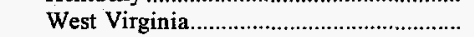 & 630.0 & 12,446 & .72 & 11.76 & 161.8 & 40.28 \\
\hline Wyoming & 317.0 & 8,784 & 27 & 5.09 & 106.1 & 18.64 \\
\hline 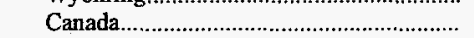 & 57.0 & 11,005 & .23 & 10.28 & 149.9 & 32.99 \\
\hline Total & $1,271.0$ & 11,499 & .60 & 9.29 & 154.0 & 35.41 \\
\hline 1995 & & & & & & \\
\hline 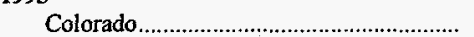 & 44.0 & 11,818 & .48 & 8.10 & 149.3 & 35.29 \\
\hline Kentucky & 220.0 & 12,840 & .72 & 7.59 & 170.2 & 43.70 \\
\hline West Virginia & 412.0 & 12,292 & .78 & 12.35 & 154.3 & 37.93 \\
\hline Wyoming & 614.0 & 8,766 & .26 & 5.11 & 105.6 & 18.51 \\
\hline Total & $1,290.0$ & 10,691 & .51 & 7.95 & 138.4 & 29.58 \\
\hline 1996 & & & & & & \\
\hline 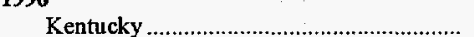 & 351.0 & 12,742 & .82 & 8.24 & 159.6 & 40.67 \\
\hline West Virginia & 332.0 & 12,176 & .85 & 13.10 & 141.2 & 34.40 \\
\hline 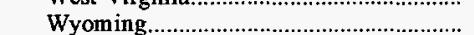 & 638.0 & 8,783 & .26 & 4.94 & 104.4 & 18.34 \\
\hline Total & $1,321.0$ & 10,688 & .56 & 7.87 & 132.4 & 28.31 \\
\hline 1997 & & & & & & \\
\hline 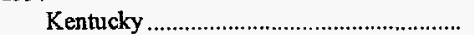 & 347.0 & 12,800 & .83 & 8.49 & 144.6 & 37.03 \\
\hline Pennsylvania & 20.0 & 13,246 & 1.56 & 6.60 & 123.3 & 32.66 \\
\hline 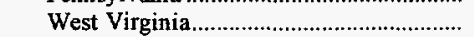 & 342.0 & 12,199 & .85 & 12.62 & 142.9 & 34.86 \\
\hline Wyoming & 675.0 & 8,789 & .30 & 5.01 & 102.9 & 18.09 \\
\hline Total & $1,384.0$ & 10,702 & .59 & 7.79 & 127.1 & 27.19 \\
\hline 1998 & & & & & & \\
\hline January - March & & & & & & \\
\hline 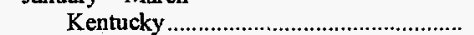 & 47.0 & 12,930 & .83 & 8.51 & 138.7 & 35.88 \\
\hline West Virginia & 125.0 & 13,038 & .74 & 8.32 & 141.3 & 36.85 \\
\hline Wyoming & 151.0 & 8,788 & .29 & 5.17 & 97.6 & 17.16 \\
\hline Total & 323.0 & 11,035 & .54 & 6.87 & 124.6 & 27.51 \\
\hline April - June & & & & & & \\
\hline 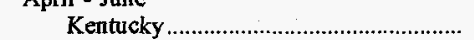 & 116.0 & 13,202 & .86 & 8.37 & 140.0 & 36.96 \\
\hline West Virginia & 84.0 & 12,995 & .69 & 8.71 & 141.7 & 36.84 \\
\hline Wyoming & 234.0 & 8,741 & 32 & 5.19 & $\begin{array}{r}141.7 \\
93.8\end{array}$ & 16.39 \\
\hline Total & 434.0 & 10,757 & .54 & 6.72 & 120.1 & 25.85 \\
\hline
\end{tabular}

See footnotes at the end of Table A7. 
Table A7. Cost and Quality of All Coal Received at Electric Utility Plants that Import Coal by Origin, 1992-1998 (Continued)

\begin{tabular}{|c|c|c|c|c|c|c|}
\hline \multirow[b]{2}{*}{$\begin{array}{l}\text { Time Period and State } \\
\text { or Country of Origin }\end{array}$} & \multirow[b]{2}{*}{$\begin{array}{l}\text { Quantity } \\
\text { (thousand } \\
\text { short tons) }\end{array}$} & \multicolumn{3}{|c|}{ Average Quality 1} & \multicolumn{2}{|c|}{ Average Cost Delivered } \\
\hline & & $\begin{array}{c}\text { Btu } \\
\text { per Pound }\end{array}$ & $\begin{array}{c}\text { Sulfur } \\
\text { Percent } \\
\text { by Weight }\end{array}$ & $\begin{array}{c}\text { Ash } \\
\text { Percent } \\
\text { by Weight }\end{array}$ & $\begin{array}{l}\text { Cents per } \\
\text { Million Btu }\end{array}$ & $\begin{array}{l}\text { Dollars per } \\
\text { Short Ton }\end{array}$ \\
\hline & \multicolumn{6}{|c|}{ Company and Plant: Detroit Edison Co, River Rouge } \\
\hline \multicolumn{7}{|l|}{1998} \\
\hline 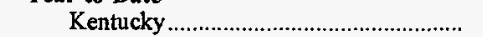 & 163.0 & 13,124 & 0.85 & 8.41 & 139.6 & 36.65 \\
\hline West Virginia & 209.0 & 13,021 & .72 & 8.48 & 141.5 & 36.85 \\
\hline \multirow{3}{*}{ Wyoming } & 385.0 & 8,760 & 31 & 5.18 & 95.3 & 16.69 \\
\hline & 757.0 & 10,876 & .54 & 6.79 & 122.1 & 26.55 \\
\hline & \multicolumn{6}{|c|}{ Company and Plant: Florida Power Corp, IMT Transfer } \\
\hline \multicolumn{7}{|l|}{1992} \\
\hline Kentucky & $1,183.1$ & 12,423 & 0.86 & 8.98 & 170.0 & 42.23 \\
\hline 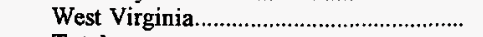 & 195.7 & 12,633 & .80 & 9.90 & 167.1 & 42.23 \\
\hline Total & $1,378.8$ & 12,452 & .85 & 9.11 & 169.6 & 42.23 \\
\hline \multicolumn{7}{|l|}{1993} \\
\hline Kentucky & 612.5 & 12,469 & .86 & 9.30 & 167.3 & 41.72 \\
\hline 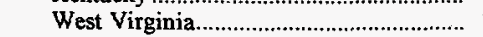 & 383.9 & 12,568 & .69 & 9.19 & 168.6 & 42.38 \\
\hline 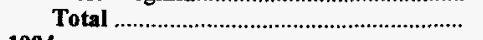 & 996.4 & 12,507 & .79 & 9.26 & 167.8 & 41.98 \\
\hline \multicolumn{7}{|l|}{1994} \\
\hline 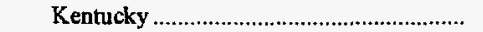 & 677.2 & 12,429 & .83 & 9.69 & 181.1 & 45.01 \\
\hline West Virginia & 658.5 & 12,552 & .71 & 9.50 & 173.0 & 43.43 \\
\hline 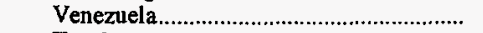 & 84.4 & 12,778 & .64 & 6.50 & 156.3 & 39.93 \\
\hline 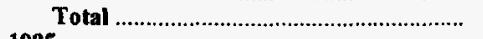 & $1,420.1$ & 12,507 & .77 & 9.41 & 175.8 & 43.97 \\
\hline \multicolumn{7}{|l|}{1995} \\
\hline 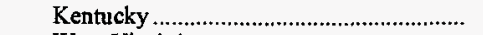 & 739.7 & 12,496 & .75 & 9.01 & 170.4 & 42.59 \\
\hline 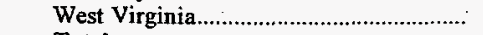 & 546.2 & 12,502 & .75 & 9.48 & 173.9 & 43.48 \\
\hline Total & $1,285.8$ & 12,498 & .75 & 9.21 & 171.9 & 42.97 \\
\hline 1996 & & & & & & \\
\hline 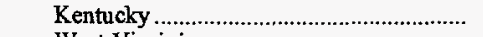 & 985.4 & 12,582 & .68 & 8.63 & 166.8 & 41.96 \\
\hline 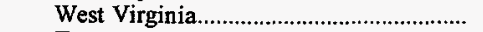 & 969.8 & 12,571 & .70 & 9.94 & 174.9 & 43.97 \\
\hline Total & $1,955.2$ & 12,576 & .69 & 9.28 & 170.8 & 42.96 \\
\hline 1997 & & & & & & \\
\hline Colorado & 14.4 & 11,131 & .60 & 7.74 & 182.5 & 40.63 \\
\hline Kentucky & 813.0 & 12,461 & .66 & 9.19 & 166.8 & 41.57 \\
\hline 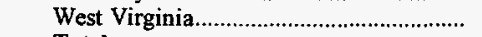 & $1,182.1$ & 12,588 & .70 & 10.23 & 177.5 & 44.68 \\
\hline Total & $2,009.6$ & 12,526 & .68 & 9.79 & 173.2 & 43.39 \\
\hline 1998 & & & & & & \\
\hline January - March & & & & & & \\
\hline 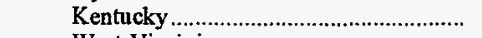 & 269.5 & 12,651 & .66 & 8.89 & 170.5 & 43.14 \\
\hline 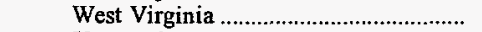 & 191.1 & 12,469 & .70 & 10.29 & 173.5 & 43.27 \\
\hline Venezuela & 61.2 & 12,976 & .71 & 5.58 & 176.5 & 45.81 \\
\hline 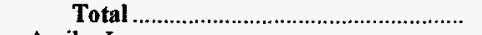 & 521.8 & 12,623 & .68 & 9.01 & 172.3 & 43.50 \\
\hline April - June & & & & & & \\
\hline 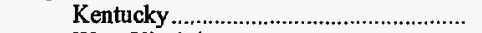 & 222.4 & 12,564 & .67 & 9.33 & 163.7 & 41.14 \\
\hline West Virginia & 256.5 & 12,389 & .71 & 10.25 & 171.0 & 42.37 \\
\hline 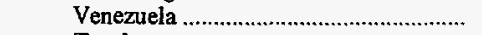 & 18.5 & 12,940 & .79 & 5.97 & 135.2 & 34.98 \\
\hline Total & 497.4 & 12,488 & .69 & 9.68 & 166.3 & 41.55 \\
\hline Year to Date & & & & & & \\
\hline 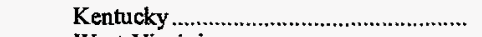 & 491.9 & 12,612 & .66 & 9.09 & 167.5 & 42.24 \\
\hline 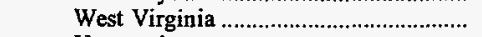 & 447.6 & 12,423 & .70 & 10.27 & 172.1 & 42.76 \\
\hline 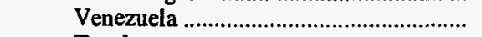 & 79.8 & 12,968 & .73 & 5.67 & 166.9 & 43.30 \\
\hline Total & $1,019.2$ & 12,557 & .69 & 9.34 & 169.4 & $\mathbf{4 2 . 5 5}$ \\
\hline & & & pany and $P$ & alf Power, $C$ & & \\
\hline 1992 & & & & & & \\
\hline Alabama & 71.9 & 12,060 & 2.75 & 12.94 & 120.6 & 29.09 \\
\hline Ilinois & $1,779.8$ & 11,926 & 2.30 & 8.37 & 180.8 & 43.12 \\
\hline 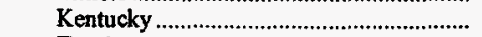 & 225.8 & 12,062 & 2.73 & 8.38 & 121.4 & 29.28 \\
\hline Total & $2,077.5$ & 11,945 & 2.71 & $\mathbf{8 . 5 3}$ & 172.2 & 41.13 \\
\hline ( & & & & & & \\
\hline Alabama & 72.3 & 12,337 & 2.09 & 11.73 & 191.1 & 47.15 \\
\hline Iflinois & $1,490.3$ & 11,992 & 2.59 & 8.15 & 176.3 & 42.27 \\
\hline 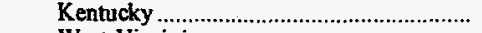 & 55.2 & 12,127 & 2.79 & 9.28 & 123.6 & 29.98 \\
\hline 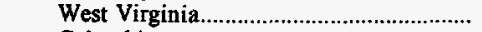 & 13.1 & 13,311 & 2.14 & 6.16 & 209.3 & 55.73 \\
\hline Colombia & 280.2 & 11,983 & .59 & 5.53 & 188.5 & 45.18 \\
\hline Venezuela & 234.8 & 12,992 & .59 & 6.11 & 172.2 & 44.75 \\
\hline Total & $2,145.9$ & 12,124 & 2.10 & 7.72 & 176.7 & 42.85 \\
\hline
\end{tabular}

See footnotes at the end of Table A7. 
Table A7. Cost and Quality of All Coal Received at Electric Utility Plants that Import Coal by Origin, 1992-1998 (Continued)

\begin{tabular}{|c|c|c|c|c|c|c|}
\hline \multirow[b]{2}{*}{$\begin{array}{l}\text { Time Period and State } \\
\text { or Country of Origin }\end{array}$} & \multirow{2}{*}{$\begin{array}{l}\text { Quantity } \\
\text { (thousand } \\
\text { short tons) }\end{array}$} & \multicolumn{3}{|c|}{ Average Quality 1} & \multicolumn{2}{|c|}{ Average Cost Delivered } \\
\hline & & $\begin{array}{c}\text { Btu } \\
\text { per Pound }\end{array}$ & $\begin{array}{c}\text { Sulfur } \\
\text { Percent } \\
\text { by Weight }\end{array}$ & $\begin{array}{c}\text { Ash } \\
\text { Percent } \\
\text { by Weight }\end{array}$ & $\begin{array}{l}\text { Cents per } \\
\text { Miltion Btu }\end{array}$ & $\begin{array}{l}\text { Dollars per } \\
\text { Short Ton }\end{array}$ \\
\hline & \multicolumn{6}{|c|}{ Company and Plant: Gulf Power, Crist } \\
\hline \multicolumn{7}{|l|}{1994} \\
\hline Alabama & 1.5 & 12,241 & 2.87 & 10.00 & 204.1 & 49.97 \\
\hline 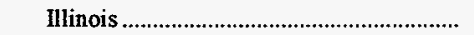 & $1,568.9$ & 11,887 & 2.15 & 7.55 & 173.1 & 41.16 \\
\hline West Virginia.............................................. & 20.7 & 13,461 & 1.08 & 5.40 & 185.8 & 50.02 \\
\hline 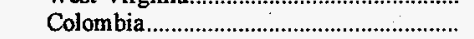 & 29.8 & 12,239 & .59 & 5.30 & 160.9 & 39.38 \\
\hline Venezuela & 283.4 & 12,252 & 1.03 & 6.28 & 216.9 & 53.15 \\
\hline Total & $1,904.4$ & 11,964 & 1.95 & 7.31 & 179.8 & 43.02 \\
\hline \multicolumn{7}{|l|}{1995} \\
\hline 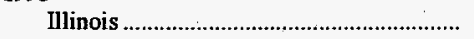 & 796.8 & 12,346 & .95 & 6.34 & 228.4 & 56.40 \\
\hline 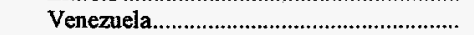 & 776.7 & 12,363 & .92 & 6.29 & 230.9 & 57.09 \\
\hline Total & $1,573.6$ & 12,354 & .93 & 6.31 & 229.6 & 56.74 \\
\hline \multicolumn{7}{|l|}{1996} \\
\hline 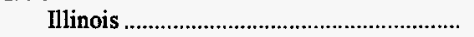 & $1,265.4$ & 12,124 & 1.10 & 6.30 & 223.6 & 54.21 \\
\hline 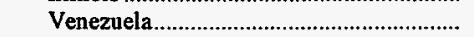 & 205.9 & 12,224 & .95 & 5.90 & 230.6 & 56.37 \\
\hline Total & $1,471.4$ & 12,138 & 1.08 & 6.25 & 224.6 & 54.52 \\
\hline \multicolumn{7}{|l|}{1997} \\
\hline Alabama & 171.8 & 12,080 & 1.04 & 12.76 & 175.1 & 42.30 \\
\hline Illinois & $1,064.6$ & 12,016 & 1.06 & 6.86 & 212.8 & 51.15 \\
\hline 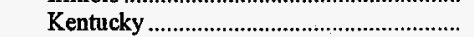 & 160.6 & 12,277 & 1.14 & 10.23 & 203.5 & 49.98 \\
\hline 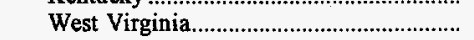 & 310.8 & 12,231 & .93 & 12.85 & 199.2 & 48.73 \\
\hline Total & $1,707.8$ & 12,086 & 1.04 & 8.86 & 205.6 & 49.71 \\
\hline \multicolumn{7}{|l|}{1998} \\
\hline \multicolumn{7}{|l|}{ January - March } \\
\hline Alabama & 22.6 & 11,717 & 1.21 & 14.04 & 187.7 & 43.98 \\
\hline 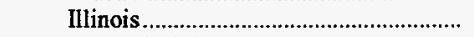 & 329.7 & 12,165 & 1.13 & 6.56 & 199.2 & 48.46 \\
\hline 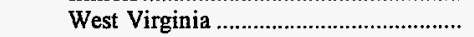 & 67.9 & 12,293 & .98 & 9.10 & 205.6 & 50.55 \\
\hline 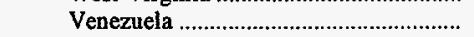 & 54.2 & 12,741 & .74 & 7.10 & 148.3 & 37.79 \\
\hline Total & 474.4 & 12,228 & 1.07 & 7.34 & 193.5 & 47.33 \\
\hline \multicolumn{7}{|l|}{ April - June } \\
\hline Alabama & 72.6 & 13,066 & .73 & 8.30 & 152.5 & 39.84 \\
\hline Illinois & 573.2 & 12,070 & 1.20 & 6.74 & 151.6 & 36.59 \\
\hline 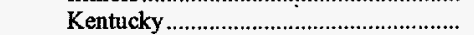 & 33.5 & 13,021 & 1.23 & 6.88 & 168.3 & 43.82 \\
\hline West Virginia & 28.7 & 12,796 & .87 & 9.75 & 142.5 & 36.46 \\
\hline Colombia & 43.6 & 12,481 & .55 & 4.50 & 151.0 & 37.69 \\
\hline 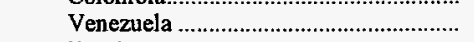 & 52.8 & 12,441 & .88 & 6.47 & 146.4 & 36.42 \\
\hline Total & 804.4 & 12,272 & 1.09 & 6.86 & 151.7 & 37.23 \\
\hline \multicolumn{7}{|l|}{ Year to Date } \\
\hline (n) & 95.1 & 12,746 & .85 & 9.66 & 160.1 & 40.82 \\
\hline 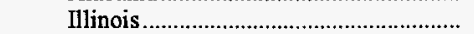 & 902.9 & 12,105 & 1.18 & 6.67 & 169.0 & 40.93 \\
\hline 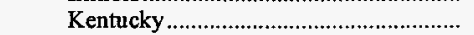 & 33.5 & 13,021 & 1.23 & 6.88 & 168.3 & 43.82 \\
\hline 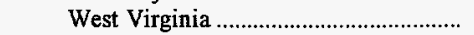 & 96.7 & 12,443 & .94 & 9.29 & 186.3 & 46.36 \\
\hline 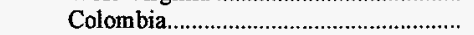 & 43.6 & 12,481 & .55 & 4.50 & 151.0 & 37.69 \\
\hline 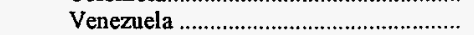 & 107.0 & 12,593 & .81 & 6.79 & 147.4 & 37.12 \\
\hline Total & $1,278.8$ & 12,256 & 1.08 & 7.04 & 167.2 & 40.98 \\
\hline & & & any and Pla & Power, Scho & & \\
\hline 1992 & & & & & & \\
\hline 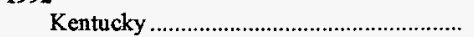 & 31.7 & 12,192 & 3.06 & 8.84 & 148.7 & 36.27 \\
\hline Total & 31.7 & 12,192 & 3.06 & 8.84 & 148.7 & 36.27 \\
\hline 1993 & & & & & & \\
\hline 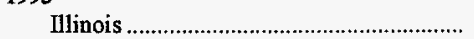 & 8.2 & 12,061 & 2.38 & 7.60 & 154.1 & 37.17 \\
\hline Kentucky & 47.9 & 12,057 & 3.10 & 8.74 & 159.5 & 38.45 \\
\hline Colombia & 7.5 & 12,170 & .62 & 7.50 & 164.4 & 40.01 \\
\hline Venezuela & 16.0 & 12,958 & .58 & 6.10 & 170.6 & 44.20 \\
\hline Total & 79.6 & 12,249 & 2.29 & 7.98 & 161.7 & 39.62 \\
\hline 1994 & & & & & & \\
\hline Kentucky & 67.1 & 11,861 & 3.09 & 9.35 & 168.7 & 40.03 \\
\hline Total & 67.1 & 11,861 & 3.09 & 9.35 & 168.7 & 40.03 \\
\hline 1995 & & & & & & \\
\hline 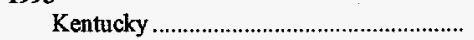 & 60.5 & 12,585 & 2.74 & 8.07 & 152.1 & 38.28 \\
\hline 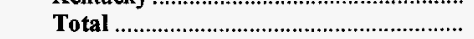 & 60.5 & 12,585 & 2.74 & 8.07 & 152.1 & 38.28 \\
\hline 1996 & & & & & & \\
\hline 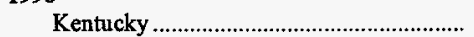 & 78.7 & 12,008 & 3.13 & 9.52 & 140.1 & 33.65 \\
\hline Total & 78.7 & 12,008 & 3.13 & 9.52 & 140.1 & 33.65 \\
\hline
\end{tabular}

See footnotes at the end of Table A7. 
Table A7. Cost and Quality of All Coal Received at Electric Utility Plants that Import Coal by Origin, 1992-1998 (Continued)

\begin{tabular}{|c|c|c|c|c|c|c|}
\hline \multirow[b]{2}{*}{$\begin{array}{l}\text { Time Period and State } \\
\text { or Country of Origin }\end{array}$} & \multirow[b]{2}{*}{$\begin{array}{l}\text { Quantity } \\
\text { (bousand } \\
\text { short tons) }\end{array}$} & \multicolumn{3}{|c|}{ Average Quality 1} & \multicolumn{2}{|c|}{ Average Cost Delivered } \\
\hline & & $\begin{array}{c}\text { Btu } \\
\text { per Pound }\end{array}$ & $\begin{array}{l}\text { Sulfur } \\
\text { Percent } \\
\text { by Weight }\end{array}$ & $\begin{array}{c}\text { Ash } \\
\text { Percent } \\
\text { by Weight }\end{array}$ & $\begin{array}{l}\text { Cents per } \\
\text { Mitlion Bta }\end{array}$ & $\begin{array}{l}\text { Doltars per } \\
\text { Short Ton }\end{array}$ \\
\hline . & \multicolumn{6}{|c|}{ Company and Plant: Gulf Power, Scholtz } \\
\hline \multicolumn{7}{|l|}{1997} \\
\hline \multicolumn{6}{|l|}{1998} & $\begin{array}{l}41.50 \\
41.50\end{array}$ \\
\hline 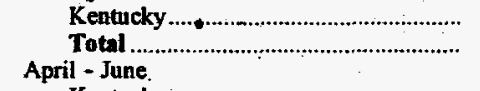 & $\begin{array}{l}6.6 \\
6.6\end{array}$ & $\begin{array}{l}12,571 \\
12,571\end{array}$ & $\begin{array}{l}2.90 \\
2.90\end{array}$ & $\begin{array}{l}8.10 \\
8.10\end{array}$ & $\begin{array}{l}165.0 \\
165.0\end{array}$ & $\begin{array}{l}41.48 \\
41.48\end{array}$ \\
\hline $\begin{array}{l}\text { Kentucky } \\
\text { Total } \\
\text { Year to Date }\end{array}$ & $\begin{array}{l}46.7 \\
46.7\end{array}$ & $\begin{array}{l}12,865 \\
12,865\end{array}$ & $\begin{array}{l}1.03 \\
1.03\end{array}$ & $\begin{array}{l}7.41 \\
7.41\end{array}$ & $\begin{array}{l}154.6 \\
154.6\end{array}$ & $\begin{array}{l}39.77 \\
39.77\end{array}$ \\
\hline \multirow{2}{*}{$\begin{array}{l}\text { Year to Date } \\
\text { Kentucky } \\
\text { Total }\end{array}$} & $\begin{array}{l}53.3 \\
\mathbf{5 3 . 3}\end{array}$ & $\begin{array}{l}12,829 \\
12,829\end{array}$ & $\begin{array}{l}1.26 \\
1.26\end{array}$ & $\begin{array}{r}7.50 \\
7.50\end{array}$ & $\begin{array}{l}155.8 \\
155.8\end{array}$ & $\begin{array}{l}39.98 \\
39.98\end{array}$ \\
\hline & \multicolumn{6}{|c|}{ Company and Plant: Gulf Power, Smith } \\
\hline \multicolumn{7}{|l|}{1992} \\
\hline 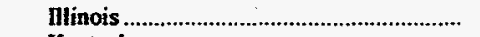 & 878.5 & 11,996 & 2.80 & 8.46 & 222.5 & 53.39 \\
\hline 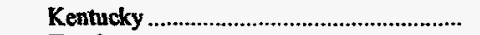 & 6.3 & 11,982 & 2.54 & 7.10 & 129.5 & 31.03 \\
\hline 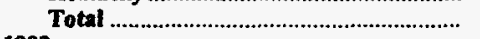 & 884.8 & 11,996 & 2.80 & 8.45 & 221.9 & $\mathbf{5 3 . 2 3}$ \\
\hline \multicolumn{7}{|l|}{1993} \\
\hline Illinois & 704.8 & 11,905 & 2.18 & 7.96 & 179.4 & 42.71 \\
\hline 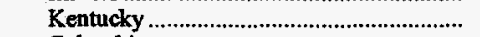 & 15.9 & 12,269 & 2.96 & 9.45 & 121.7 & 29.85 \\
\hline 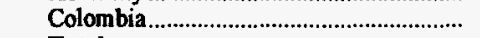 & 198.2 & 11,823 & .61 & 5.96 & 184.6 & 43.65 \\
\hline 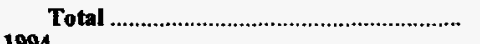 & 918.9 & 11,893 & 1.85 & 7.55 & 179.5 & 42.69 \\
\hline \multicolumn{7}{|l|}{1994} \\
\hline 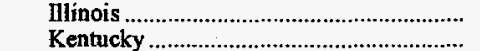 & 391.8 & 12,086 & 2.11 & 7.93 & 160.3 & 38.76 \\
\hline 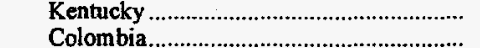 & 17.7 & 11,881 & 3.22 & 10.78 & 140.2 & 33.31 \\
\hline & 286.6 & 12,299 & .61 & 4.17 & 172.3 & 42.39 \\
\hline & 127.3 & 11,318 & .65 & 12.60 & 181.1 & 41.00 \\
\hline 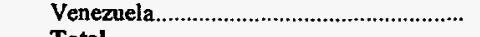 & 53.8 & 12,272 & .96 & 6.52 & 229.1 & 56.24 \\
\hline 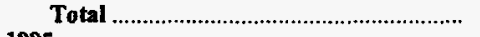 & 877.3 & 12,051 & 1.36 & 7.35 & 171.1 & 41.23 \\
\hline \multicolumn{7}{|l|}{1995} \\
\hline Illinois & 981.7 & 11,728 & 2.26 & 8.25 & 143.5 & 33.67 \\
\hline Venezuela & 114.6 & 12,202 & 1.00 & 6.52 & 236.1 & 57.63 \\
\hline Total & $1,096.4$ & 11,777 & 2.13 & 8.07 & 153.6 & 36.17 \\
\hline \multicolumn{7}{|l|}{1996} \\
\hline 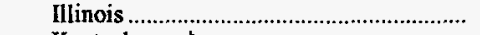 & 710.6 & 11,792 & 1.99 & 7.97 & 158.1 & 37.28 \\
\hline 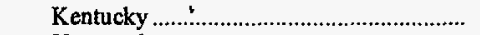 & 285.8 & 11,895 & 2.87 & 7.68 & 195.0 & 46.40 \\
\hline 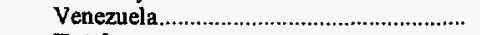 & 92.2 & 12,171 & .99 & 6.03 & 234.7 & 57.13 \\
\hline Total & $1,088.7$ & 11,851 & 2.13 & 7.73 & 174.5 & 41.35 \\
\hline \multicolumn{7}{|l|}{1997} \\
\hline Alabama & 190.6 & 11,712 & 2.92 & 13.90 & 179.6 & 42.08 \\
\hline 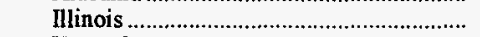 & 289.4 & 12,019 & 2.04 & 7.44 & 203.7 & 48.97 \\
\hline 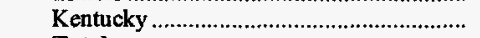 & 460.6 & 12,021 & 2.87 & 9.56 & 183.7 & 44.16 \\
\hline Total & 940.6 & 11,958 & 2.62 & 9.78 & 189.1 & 45.22 \\
\hline \multirow{2}{*}{\multicolumn{7}{|c|}{1998}} \\
\hline January - March & & & & & & \\
\hline Alabama & 13.8 & 11,636 & 2.94 & 14.81 & 174.4 & 40.58 \\
\hline 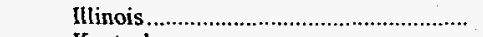 & 67.0 & 12,431 & 2.61 & 8.61 & 154.9 & 38.51 \\
\hline 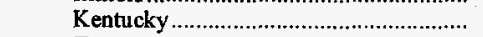 & 169.7 & 11,952 & 2.60 & 9.61 & 162.0 & 38.74 \\
\hline 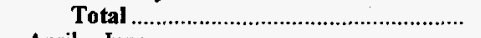 & 250.5 & 12,063 & 2.62 & 9.63 & 160.7 & 38.78 \\
\hline April - June & & & & & & \\
\hline 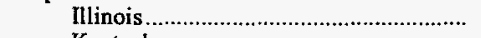 & 59.8 & 12,657 & 2.63 & 8.98 & 140.4 & 35.55 \\
\hline 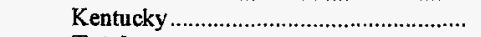 & 143.7 & 11,933 & 2.75 & 8.82 & 139.2 & 33.22 \\
\hline 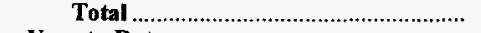 & 203.5 & 12,146 & 2.72 & 8.87 & 139.6 & 33.90 \\
\hline Year to Date & & & & & & \\
\hline Alabama & 13.8 & 11,636 & 2.94 & 14.81 & 174.4 & 40.58 \\
\hline Illinois & 126.8 & 12,538 & 2.62 & 8.78 & 148.0 & 37.11 \\
\hline Kentucky .............................................. & 313.4 & 11,944 & 2.67 & 9.24 & 151.6 & 36.21 \\
\hline Total & 454.0 . & 12,100 & 2.66 & 9.28 & 151.2 & 36.59 \\
\hline & & Company & lant: Holyol & Power (NL & nt Tom & \\
\hline 1992 & & & & & & \\
\hline Pennsylvania & 354.8 & 13,234 & 1.34 & 6.26 & 168.2 & 44.51 \\
\hline West Virginia......................................... & 8.1 & 12,800 & .80 & 8.50 & 198.2 & 50.74 \\
\hline Total & 362.9 & 13,224 & 1.33 & 6.31 & 168.8 & 44.65 \\
\hline
\end{tabular}

See footnotes at the end of Table A7. 
Table A7. Cost and Quality of All Coal Received at Electric Utility Plants that Import Coal by Origin, 1992-1998 (Continued)

\begin{tabular}{|c|c|c|c|c|c|c|}
\hline \multirow[b]{2}{*}{$\begin{array}{l}\text { Time Period and State } \\
\text { or Country of Origin }\end{array}$} & \multirow[b]{2}{*}{$\begin{array}{l}\text { Quantity } \\
\text { (thousand } \\
\text { short tons) }\end{array}$} & \multicolumn{3}{|c|}{ Average Qualityl } & \multicolumn{2}{|c|}{ Average Cost Delivered } \\
\hline & & $\begin{array}{c}\text { Bto } \\
\text { per Pound }\end{array}$ & $\begin{array}{c}\text { Sulfar } \\
\text { Percent } \\
\text { by Weight }\end{array}$ & $\begin{array}{c}\text { Ash } \\
\text { Percent } \\
\text { by Weight }\end{array}$ & $\begin{array}{l}\text { Cents per } \\
\text { Million Btu }\end{array}$ & $\begin{array}{l}\text { Dollars per } \\
\text { Short Ton }\end{array}$ \\
\hline & \multicolumn{6}{|c|}{ Company and Plant: Holyoke Water Power (NU), Mount Tom } \\
\hline \multicolumn{7}{|l|}{1993} \\
\hline 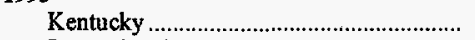 & 7.3 & 13,132 & 0.75 & 7.50 & 195.9 & 51.45 \\
\hline 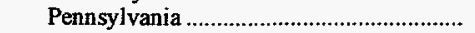 & 299.9 & 13,201 & 1.52 & 6.34 & 164.7 & 43.49 \\
\hline 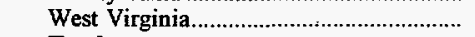 & 7.0 & 13,087 & .91 & 7.60 & 171.7 & 44.94 \\
\hline Total & 314.2 & 13,197 & 1.49 & 6.39 & 165.6 & 43.71 \\
\hline \multicolumn{7}{|l|}{1994} \\
\hline Kentucky & 47.8 & 12,884 & .55 & 7.74 & 206.0 & 53.07 \\
\hline 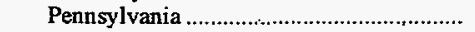 & 289.2 & 13,171 & 1.48 & 6.60 & 156.8 & 41.31 \\
\hline 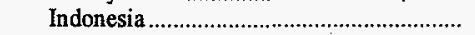 & 7.9 & 12,651 & .43 & 3.30 & 195.4 & 49.44 \\
\hline Total & 344.9 & 13,119 & 1.33 & 6.68 & 164.4 & 43.13 \\
\hline \multicolumn{7}{|l|}{1995} \\
\hline 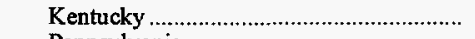 & 157.3 & 13,053 & .52 & 7.40 & 193.3 & 50.47 \\
\hline 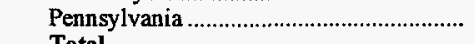 & 212.5 & 13,227 & 1.37 & 7.20 & 156.9 & 41.50 \\
\hline Total & 369.8 & 13,153 & 1.01 & $\mathbf{7 . 2 8}$ & 172.3 & 45.31 \\
\hline \multicolumn{7}{|l|}{1996} \\
\hline Kentucky & 135.8 & 13,081 & .51 & 7.78 & 198.3 & 51.88 \\
\hline 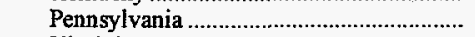 & 225.0 & 13,301 & 1.38 & 6.91 & 159.2 & 42.35 \\
\hline 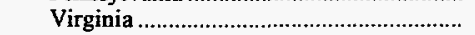 & 1.9 & 14,243 & .80 & 5.20 & 212.3 & 60.48 \\
\hline West Virginia & 8.4 & 13,227 & .64 & 8.20 & 189.1 & 50.02 \\
\hline Total & 371.1 & 13,224 & 1.04 & $\mathbf{7 . 2 5}$ & 174.3 & 46.10 \\
\hline \multicolumn{7}{|l|}{1997} \\
\hline 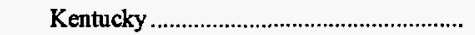 & 126.8 & 13,006 & .55 & 7.61 & 203.8 & 53.01 \\
\hline 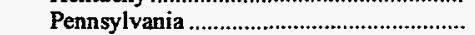 & 280.1 & 13,248 & 1.38 & 7.15 & 165.9 & 43.96 \\
\hline 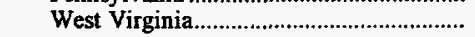 & 42.1 & 13,168 & .61 & 7.66 & 194.8 & 51.30 \\
\hline Total & 449.1 & 13,172 & 1.07 & 7.33 & 179.2 & $\mathbf{4 7 . 2 0}$ \\
\hline \multirow{2}{*}{\multicolumn{7}{|c|}{$\begin{array}{l}1998 \\
\text { January - March }\end{array}$}} \\
\hline & & & & & & \\
\hline Kentucky & 37.1 & 13,070 & 1.12 & 7.52 & 200.6 & 52.44 \\
\hline 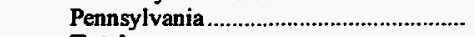 & 49.3 & 13,220 & 1.49 & 7.40 & 160.8 & 42.51 \\
\hline 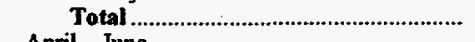 & 86.3 & 13,155 & 1.33 & 7.45 & 177.8 & 46.77 \\
\hline & & & & & & \\
\hline 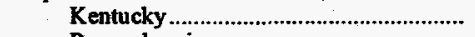 & 69.3 & 13,002 & .51 & 7.26 & 199.1 & 51.78 \\
\hline Pennsylvania & 38.1 & 13,212 & 1.68 & 7.24 & 161.5 & 42.67 \\
\hline 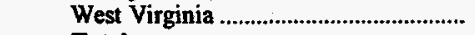 & 1.6 & 13,456 & .68 & 6.75 & 171.9 & 46.28 \\
\hline 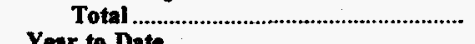 & 109.1 & 13,082 & .92 & 7.25 & 185.4 & 48.52 \\
\hline $\begin{array}{l}\text { Year to Date } \\
\text { Kentucky }\end{array}$ & & & & & & \\
\hline 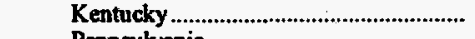 & 106.4 & 13,025 & .72 & 7.35 & 199.7 & 52.01 \\
\hline Pennsylvania & 87.4 & 13,216 & 1.57 & 7.33 & 161.1 & 42.58 \\
\hline West Virginia ............................................... & 1.6 & 13,456 & .68 & 6.75 & 171.9 & 46.28 \\
\hline 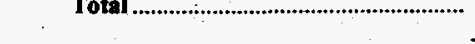 & 195.4 & 13,114 & 1.10 & 7.34 & 182.0 & 47.75 \\
\hline & & Company and & Jacksonvill & ic Authority, & Ins River & \\
\hline 1992 & & & & & & \\
\hline Kentucky & $1,563.4$ & 12,831 & 1.18 & 8.43 & 160.2 & 41.11 \\
\hline 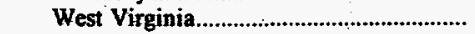 & 642.4 & 12,063 & .82 & 12.58 & 199.9 & 48.22 \\
\hline 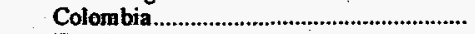 & $1,418.6$ & 11,897 & .71 & 6.91 & 150.0 & 35.70 \\
\hline Total & $3,624.4$ & 12,329 & .93 & 8.57 & 163.2 & 40.25 \\
\hline 1993 & & & & & & \\
\hline 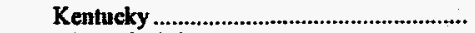 & $1,300.4$ & 12,802 & 1.30 & 8.36 & 172.0 & 44.03 \\
\hline 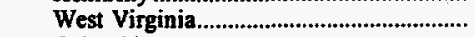 & 243.0 & 12,049 & .75 & 12.79 & 187.6 & 45.21 \\
\hline Colombia & $2,291.2$ & 11,849 & .68 & 7.21 & 136.9 & 32.44 \\
\hline Total & $3,834.6$ & 12,185 & .89 & 7.95 & 152.6 & 37.18 \\
\hline 1994 & & & & & & \\
\hline 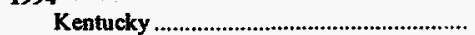 & $1,106.7$ & 12,775 & 1.27 & 8.92 & 173.2 & 44.25 \\
\hline 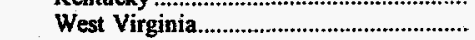 & 595.3 & 12,193 & .82 & 11.98 & 185.1 & 45.14 \\
\hline 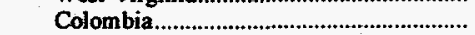 & $2,032.1$ & 11,883 & .69 & 7.40 & 135.6 & 32.22 \\
\hline 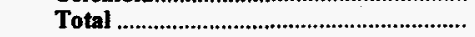 & $\mathbf{3 , 7 3 4 . 1}$ & 12,197 & .88 & 8.58 & 155.2 & 37.85 \\
\hline 1995 & & & & & & \\
\hline Kentucky & $1,695.5$ & 12,605 & 1.25 & 9.30 & 168.0 & 42.35 \\
\hline 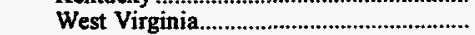 & 645.7 & 12,143 & .88 & 12.85 & 188.2 & 45.70 \\
\hline 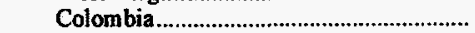 & $1,340.6$ & 11,826 & .67 & 7.52 & 151.5 & 35.82 \\
\hline 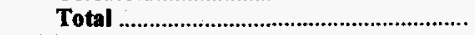 & $3,681.8$ & 12,241 & .97 & 9.28 & 165.7 & 40.56 \\
\hline 1996 & & & & & & \\
\hline 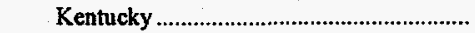 & $2,239.5$ & 12,742 & 1.31 & 9.08 & 164.4 & 41.89 \\
\hline 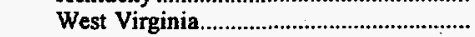 & 133.3 & 12,384 & 1.68 & 11.85 & 177.2 & 43.88 \\
\hline Colombia & $1,417.2$ & 11,810 & .66 & 7.71 & 152.9 & 36.11 \\
\hline Total & $3,790.0$ & 12,381 & 1.08 & 8.66 & 160.7 & 39.80 \\
\hline
\end{tabular}

See footnotes at the end of Table A7. 
Table A7. Cost and Quality of All Coal Received at Electric Utility Plants that Import Coal by Origin, 1992-1998 (Continued)

\begin{tabular}{|c|c|c|c|c|c|c|}
\hline \multirow[b]{2}{*}{$\begin{array}{l}\text { Time Period and State } \\
\text { or Country of Origin }\end{array}$} & \multirow[b]{2}{*}{$\begin{array}{l}\text { Quantity } \\
\text { (thousand } \\
\text { short tons) }\end{array}$} & \multicolumn{3}{|c|}{ Average Qualityl } & \multicolumn{2}{|c|}{ Average Cost Delivered } \\
\hline & & $\begin{array}{c}\text { Btu } \\
\text { per Pound }\end{array}$ & $\begin{array}{c}\text { Sulfur } \\
\text { Percent } \\
\text { by Weight }\end{array}$ & $\begin{array}{c}\text { Ash } \\
\text { Percent } \\
\text { by Weight }\end{array}$ & $\begin{array}{l}\text { Cents per } \\
\text { Million Btu }\end{array}$ & $\begin{array}{l}\text { Dollars per } \\
\text { Short Ton }\end{array}$ \\
\hline & \multicolumn{6}{|c|}{ Company and Plant: Jacksonville Electric Authority, St Johns River } \\
\hline \multicolumn{7}{|l|}{1997} \\
\hline Kentucky & $1,561.8$ & 12,614 & 1.49 & 9.99 & 174.6 & 44.05 \\
\hline 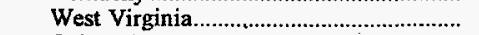 & 177.9 & 12,013 & .85 & 13.35 & 196.6 & 47.24 \\
\hline 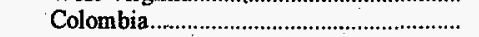 & $1,385.3$ & 11,851 & .78 & 7.42 & 150.1 & 35.59 \\
\hline 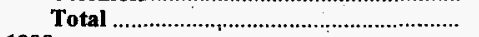 & $3,125.0$ & 12,242 & 1.14 & 9.05 & 165.3 & 40.48 \\
\hline \multicolumn{7}{|l|}{$\begin{array}{l}1998 \\
\text { January - March }\end{array}$} \\
\hline 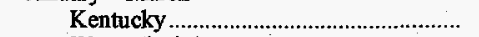 & 364.9 & 12,674 & 1.31 & 9.98 & 171.9 & 43.57 \\
\hline 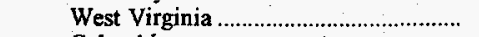 & 9.9 & 12,169 & .86 & 13.20 & 149.6 & 36.41 \\
\hline 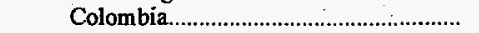 & 359.3 & 11,802 & .65 & 7.13 & 149.0 & 35.16 \\
\hline 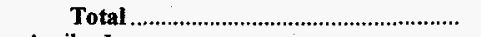 & 734.2 & 12,241 & .98 & 8.63 & 160.8 & 39.36 \\
\hline \multicolumn{7}{|l|}{ April - June } \\
\hline 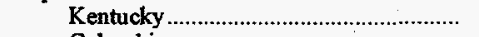 & 538.8 & 12,687 & 1.34 & 9.68 & 172.3 & 43.71 \\
\hline Colombia & 296.8 & 11,796 & .61 & 6.83 & 147.2 & 34.72 \\
\hline 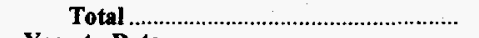 & 835.6 & 12,371 & 1.08 & 8.67 & 163.8 & 40.52 \\
\hline \multicolumn{7}{|l|}{ Year to Date } \\
\hline Kentucky & 903.7 & 12,682 & 1.33 & 9.80 & 172.1 & 43.65 \\
\hline 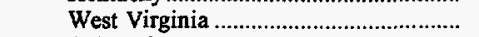 & 9.9 & 12,169 & .86 & 13.20 & 149.6 & 36.41 \\
\hline 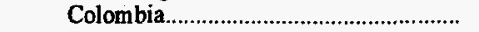 & 656.1 & 11,799 & .63 & 6.99 & 148.2 & 34.96 \\
\hline \multirow[t]{2}{*}{ Total } & $1,569.8$ & 12,310 & 1.03 & 8.65 & 162.4 & 39.98 \\
\hline & \multicolumn{6}{|c|}{ Company and Plant: Lakeland Dept of Elec Wtr Utils, Plant 3-Mcintosh } \\
\hline \multicolumn{7}{|l|}{1992} \\
\hline 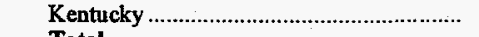 & 818.0 & 12,999 & 1.04 & 7.71 & 165.0 & 42.90 \\
\hline Total & 818.0 & 12,999 & 1.04 & 7.71 & 165.0 & 42.90 \\
\hline \multicolumn{7}{|l|}{1993} \\
\hline 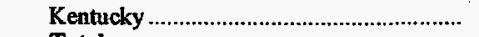 & 727.0 & 13,046 & 1.05 & 7.63 & 167.7 & 43.77 \\
\hline \multicolumn{5}{|l|}{1994} & 167.7 & 43.77 \\
\hline 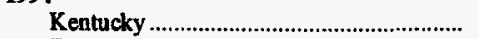 & 992.0 & 12,936 & 1.12 & 8.02 & 173.4 & 44.87 \\
\hline \multirow{2}{*}{\multicolumn{7}{|c|}{1995}} \\
\hline 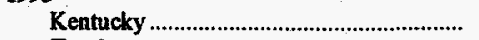 & 945.0 & 12,956 & 1.22 & 8.18 & & 44.84 \\
\hline Total & 945.0 & 12,956 & 1.22 & 8.18 & 173.0 & 44.84 \\
\hline 1996 & & & & & & \\
\hline 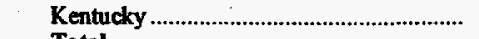 & 807.0 & 12,840 & 1.32 & 9.09 & 172.8 & 44.38 \\
\hline 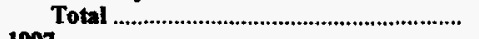 & 807.0 & 12,840 & 1.32 & 9.09 & 172.8 & 44.38 \\
\hline 1997 & & & & & & \\
\hline Kentucky & 758.0 & 12,876 & 1.26 & 8.42 & 172.7 & 44.48 \\
\hline 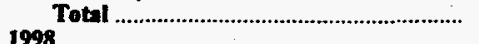 & 758.0 & 12,876 & 1.26 & 8.42 & 172.7 & 44.48 \\
\hline $\begin{array}{l}1998 \\
\text { January - March }\end{array}$ & & & & & & \\
\hline 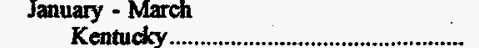 & & & & & & \\
\hline 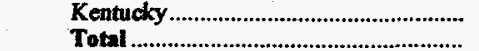 & 134.0 & 12,773 & 1.28 & 9.02 & 174.8 & 44.67 \\
\hline 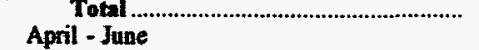 & 134.0 & 12,773 & 1.28 & 9.02 & 174.8 & 44.67 \\
\hline 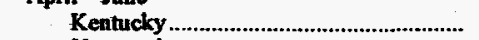 & 84.0 & 12,927 & 1.31 & & 178.7 & $46 ? 1$ \\
\hline Venezuela & 6.0 & 12,941 & .62 & 5.70 & 175.2 & $\begin{array}{l}40.21 \\
45.35\end{array}$ \\
\hline 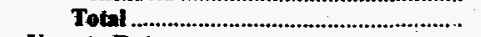 & 90.0 & 12,928 & 1.26 & 8.20 & 178.5 & 46.16 \\
\hline Year to Date & & & & & & \\
\hline Kentucky & 218.0 & 12,832 & 1.29 & 8.77 & 176.4 & 45.26 \\
\hline Venezuela .................................................. & 6.0 & 12,941 & .62 & $\begin{array}{r}5.70 \\
8.69\end{array}$ & 175.2 & 45.35 \\
\hline 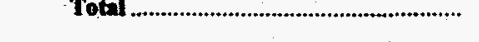 & 224.0 & 12,835 & 1.27 & 8.69 & 176.3 & $\mathbf{4 5 . 2 7}$ \\
\hline & & Company & ant: Mississi & er (Southerm & Daniel & \\
\hline 1992 & & & & & & \\
\hline 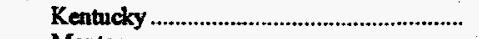 & 810.6 & 12,988 & 0.73 & 7.22 & 170.0 & 44.15 \\
\hline Montana & 82.2 & 9,383 & .30 & 4.15 & 136.0 & 25.51 \\
\hline Wyoming & 70.9 & 8,760 & .34 & 4.92 & 153.0 & 26.81 \\
\hline 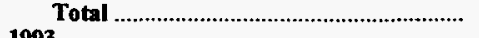 & 963.7 & 12,369 & .66 & 6.79 & 166.9 & 41.29 \\
\hline 1993 & & & & & & \\
\hline Colorado & 158.6 & 11,535 & .45 & 9.58 & 158.9 & 36.66 \\
\hline 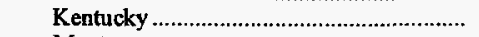 & 774.6 & 12,881 & .70 & 8.12 & 173.8 & 44.78 \\
\hline 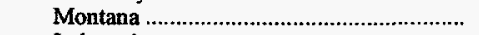 & 177.7 & 9,425 & .39 & 4.61 & 159.1 & 29.99 \\
\hline 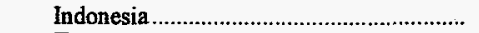 & 67.5 & 9,745 & .08 & 1.23 & 168.9 & 32.92 \\
\hline Total & $1,178.5$ & 11,999 & .58 & 7.39 & 169.9 & 40.78 \\
\hline
\end{tabular}

See footnotes at the end of Table A7. 
Table A7. Cost and Quality of All Coal Received at Electric Utility Plants that Import Coal by Origin, 1992-1998 (Continued)

\begin{tabular}{|c|c|c|c|c|c|c|}
\hline \multirow[b]{2}{*}{$\begin{array}{l}\text { Time Period and State } \\
\text { or Country of Origin }\end{array}$} & \multirow{2}{*}{$\begin{array}{l}\text { Quantity } \\
\text { (thousand } \\
\text { short tons) }\end{array}$} & \multicolumn{3}{|c|}{ Average Qualityl } & \multicolumn{2}{|c|}{ Average Cost Delivered } \\
\hline & & $\begin{array}{c}\text { Btu } \\
\text { per Pound }\end{array}$ & $\begin{array}{c}\text { Sulfur } \\
\text { Percent } \\
\text { by Weight }\end{array}$ & $\begin{array}{c}\text { Ash } \\
\text { Percent } \\
\text { by Weight }\end{array}$ & $\begin{array}{l}\text { Cents per } \\
\text { Million Btu }\end{array}$ & $\begin{array}{l}\text { Dollars per } \\
\text { Short Ton }\end{array}$ \\
\hline & \multicolumn{6}{|c|}{ Company and Plant: Mississippi Power (Southern Co), Daniel } \\
\hline \multicolumn{7}{|l|}{1994} \\
\hline Colorado & 715.2 & 11,072 & 0.43 & 10.37 & 159.5 & 35.31 \\
\hline Kentucky & 279.3 & 12,739 & .68 & 9.06 & 181.7 & 46.28 \\
\hline 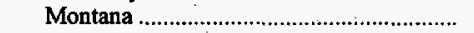 & $1,288.4$ & 9,402 & .40 & 4.78 & 138.0 & 25.96 \\
\hline Total & $2,282.8$ & 10,334 & .44 & 7.06 & 151.8 & 31.38 \\
\hline \multicolumn{7}{|l|}{1995} \\
\hline Colorado. & 951.3 & 11,076 & .42 & 9.89 & 161.4 & 35.75 \\
\hline Montana & $1,269.5$ & 9,399 & .38 & 4.43 & 140.0 & 26.31 \\
\hline 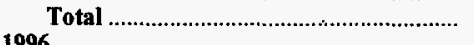 & $2,220.8$ & 10,118 & .39 & 6.77 & 150.0 & 30,36 \\
\hline \multicolumn{7}{|l|}{1996} \\
\hline Montana & $2,163.3$ & $\begin{array}{r}11,397 \\
9,394\end{array}$ & $\begin{array}{l}.46 \\
.40\end{array}$ & $\begin{array}{l}8.87 \\
4.48\end{array}$ & 141.0 & $\begin{array}{l}30.32 \\
26.49\end{array}$ \\
\hline 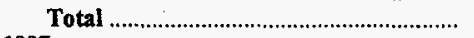 & $2,671.2$ & 9,774 & .41 & 5.31 & 145.1 & 28.36 \\
\hline \multicolumn{7}{|l|}{1997} \\
\hline Montana & $3,221.3$ & 9,323 & .39 & 4.56 & 145.5 & 27.13 \\
\hline Total & $\mathbf{3 , 2 2 1 . 3}$ & 9,323 & .39 & 4.56 & 145.5 & 27.13 \\
\hline \multicolumn{7}{|l|}{1998} \\
\hline \multicolumn{7}{|l|}{ January - March } \\
\hline Montana & 801.7 & 9,421 & .39 & 4.65 & 149.0 & 28.07 \\
\hline 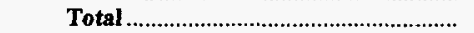 & 801.7 & 9,421 & .39 & 4.65 & 149.0 & 28.07 \\
\hline \multicolumn{7}{|l|}{ April - June } \\
\hline Montana & 804.3 & 9,395 & .37 & 4.37 & 145.8 & 27.39 \\
\hline Total & 804.3 & 9,395 & .37 & 4.37 & 145.8 & 27.39 \\
\hline Year to Date & & & & & & \\
\hline Montana & $1,606.0$ & 9,408 & .38 & 4.51 & 147.4 & 27.73 \\
\hline Total & $1,606.0$ & 9,408 & .38 & 4.51 & 147.4 & 27.73 \\
\hline & & Company : & nt: Mississip & r (Southern & latson & \\
\hline 1992 & & & & & & \\
\hline 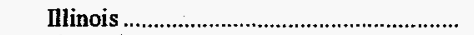 & $1,238.7$ & 12,757 & 2.74 & 8.75 & 132.7 & 33.86 \\
\hline 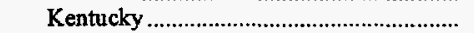 & 248.5 & 12,208 & 2.50 & 8.08 & 130.5 & 31.87 \\
\hline 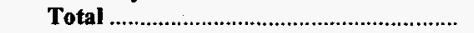 & $1,487.2$ & 12,665 & 2.70 & 8.64 & 132.4 & 33.53 \\
\hline 1993 & & & & & & \\
\hline 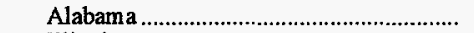 & 32.0 & 12,223 & 1.13 & 11.54 & 165.6 & 40.49 \\
\hline Illinois & $1,079.9$ & 12,697 & 2.71 & 9.10 & 134.1 & 34.06 \\
\hline Kentucky & 215.3 & 12,168 & 1.11 & 8.61 & 141.0 & 34.30 \\
\hline 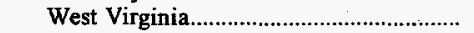 & 68.9 & 12,244 & 1.07 & 12.12 & 144.0 & 35.25 \\
\hline Total & $1,396.1$ & 12,582 & 2.35 & 9.23 & 136.3 & 34.31 \\
\hline 1994 & & & & & & \\
\hline 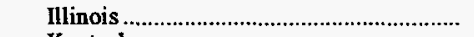 & $1,062.7$ & 12,456 & 2.41 & 8.55 & 131.8 & 32.84 \\
\hline 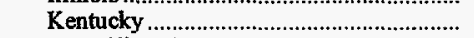 & 30.8 & 11,934 & 1.05 & 11.28 & 145.2 & 34.65 \\
\hline 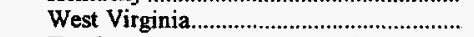 & 62.2 & 12,392 & .94 & 10.43 & 151.5 & 37.55 \\
\hline 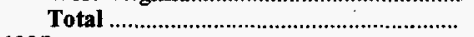 & $1,155.7$ & 12,439 & 2.30 & 8.72 & 133.2 & 33.14 \\
\hline 1995 & & & & & & \\
\hline Colorado & 11.4 & 11,972 & .50 & 7.91 & 145.8 & 34.91 \\
\hline 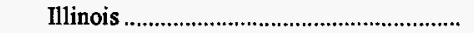 & $1,235.8$ & 12,440 & 2.35 & 8.86 & 123.0 & 30.61 \\
\hline Total & $1,247.2$ & 12,436 & 2.33 & 8.86 & 123.2 & 30.65 \\
\hline 1996 & & & & & & \\
\hline 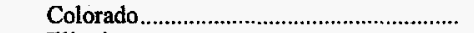 & 11.2 & 11,439 & .44 & 8.63 & 148.7 & 34.02 \\
\hline 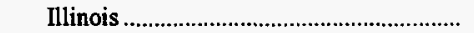 & $1,703.1$ & 12,197 & 1.70 & 7.00 & 131.3 & 32.03 \\
\hline 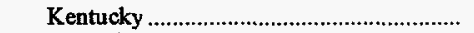 & 104.6 & 12,123 & 2.12 & 10.31 & 126.5 & 30.68 \\
\hline 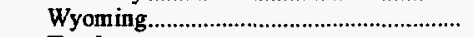 & 12.8 & 8,738 & .44 & 5.25 & 129.5 & 22.63 \\
\hline Total & $1,831.6$ & 12,164 & 1.71 & 7.19 & 131.1 & 31.90 \\
\hline 1997 & & & & & & \\
\hline 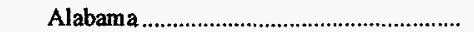 & 126.4 & 12,461 & 1.04 & 9.73 & 145.8 & 36.34 \\
\hline Colorado & 35.6 & 11,518 & .49 & 7.49 & 154.8 & 35.65 \\
\hline 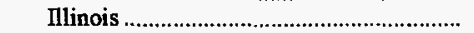 & $1,149.4$ & 11,897 & 1.19 & 7.04 & 138.4 & 32.93 \\
\hline 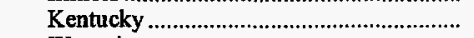 & 251.9 & 12,560 & 1.13 & 8.37 & 154.3 & 38.76 \\
\hline 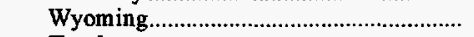 & 285.4 & 8,677 & .46 & 5.69 & 133.8 & 23.22 \\
\hline 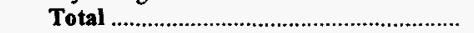 & $1,848.7$ & 11,522 & 1.04 & 7.20 & 141.1 & 32.51 \\
\hline 1998 & & & & & & \\
\hline January - March & & & & & & \\
\hline 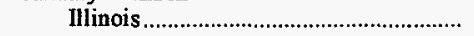 & 362.1 & 11,798 & 1.68 & 7.40 & 144.6 & 34.11 \\
\hline 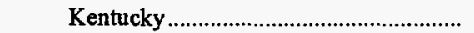 & 15.4 & 12,688 & 1.93 & 7.62 & 146.6 & 37.19 \\
\hline 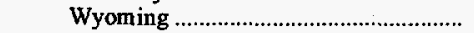 & 91.2 & 8,744 & .40 & 5.34 & 132.9 & 23.24 \\
\hline Venezuela & 13.6 & 12,312 & .71 & 5.20 & 154.1 & 37.95 \\
\hline Total & 482.3 & 11,263 & 1.42 & 6.95 & 143.2 & 32.26 \\
\hline
\end{tabular}

See footnotes at the end of Table A7. 
Table A7. Cost and Quality of All Coal Received at Electric Utility Plants that Import Coal by Origin, 1992-1998 (Continued)

\begin{tabular}{|c|c|c|c|c|c|c|}
\hline \multirow[b]{2}{*}{$\begin{array}{l}\text { Time Period and State } \\
\text { or Country of Origin }\end{array}$} & \multirow[b]{2}{*}{$\begin{array}{l}\text { Quantity } \\
\text { (thousand } \\
\text { short tons) }\end{array}$} & \multicolumn{3}{|c|}{ Average Quality 1} & \multicolumn{2}{|c|}{ Average Cost Delivered } \\
\hline & & $\begin{array}{c}\text { Btu } \\
\text { per Pound }\end{array}$ & $\begin{array}{c}\text { Sulfur } \\
\text { Percent } \\
\text { by Weight }\end{array}$ & $\begin{array}{c}\text { Ash } \\
\text { Percent } \\
\text { by Weight }\end{array}$ & $\begin{array}{c}\text { Cents per } \\
\text { Million Btu }\end{array}$ & $\begin{array}{l}\text { Dollars per } \\
\text { Short Ton }\end{array}$ \\
\hline & \multicolumn{6}{|c|}{ Company and Plant: Mississippi Power (Southern Co), Watson } \\
\hline \multicolumn{7}{|l|}{1998} \\
\hline Alabama & 28.3 & 13,083 & 0.74 & 8.50 & 148.0 & 38.73 \\
\hline 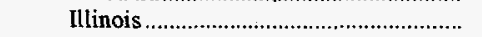 & 382.0 & 12,111 & 1.64 & 7.40 & 139.1 & 33.68 \\
\hline Wyoming & 139.8 & 8,682 & .43 & 5.18 & 132.5 & 23.00 \\
\hline 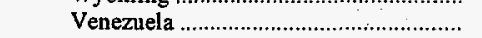 & 51.1 & 12,617 & .76 & 7.20 & 137.1 & 34.60 \\
\hline 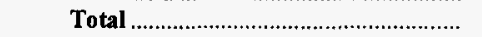 & 601.2 & 11,402 & 1.24 & 6.92 & 138.2 & 31.51 \\
\hline \multicolumn{7}{|l|}{ Year to Date } \\
\hline Alabama & 28.3 & 13,083 & .74 & 8.50 & 148.0 & 38.73 \\
\hline 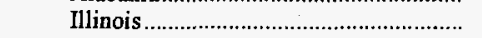 & 744.1 & 11,959 & 1.66 & 7.40 & 141.7 & 33.89 \\
\hline 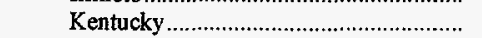 & 15.4 & 12,688 & 1.93 & 7.62 & 146.6 & 37.19 \\
\hline 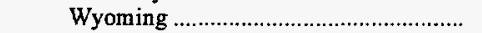 & 231.1 & 8,706 & .42 & 5.24 & 132.6 & 23.10 \\
\hline 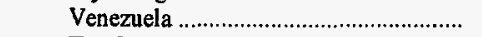 & 64.7 & 12,553 & .75 & 6.78 & 140.6 & 35.30 \\
\hline \multirow[t]{2}{*}{ Total } & $1,083.5$ & 11,340 & 1.32 & 6.93 & 140.4 & 31.85 \\
\hline & \multicolumn{6}{|c|}{ Company and Plant: Montaup Electric, Somerset } \\
\hline \multicolumn{7}{|l|}{1992} \\
\hline 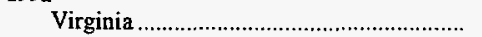 & 413.0 & 12,917 & 1.03 & 7.93 & 176.4 & 45.58 \\
\hline Total & 413.0 & 12,917 & 1.03 & 7.93 & 176.4 & 45.58 \\
\hline \multicolumn{7}{|l|}{1993} \\
\hline 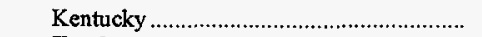 & 113.6 & 12,903 & 1.00 & 7.88 & 178.1 & 45.97 \\
\hline Total & 113.6 & 12,903 & 1.00 & 7.88 & 178.1 & 45.97 \\
\hline \multicolumn{7}{|l|}{1994} \\
\hline 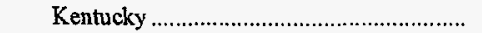 & 43.7 & 12,429 & .63 & 8.74 & 197.7 & 49.14 \\
\hline 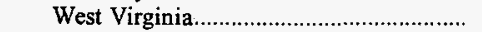 & 189.1 & 12,931 & .73 & 8.39 & 178.8 & 46.23 \\
\hline Total & 232.8 & 12,836 & .71 & 8.45 & 182.2 & 46.78 \\
\hline \multicolumn{7}{|l|}{1995} \\
\hline 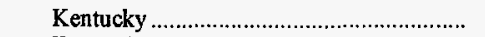 & 115.8 & 12,838 & .67 & 7.90 & 178.4 & 45.79 \\
\hline 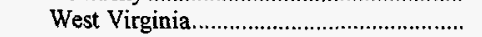 & 157.7 & 12,840 & .76 & 7.42 & 177.9 & 45.68 \\
\hline Total & 273.5 & 12,839 & .72 & 7.62 & 178.1 & 45.73 \\
\hline 1996 & & & & & & \\
\hline 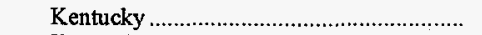 & 55.6 & 12,622 & .77 & 7.03 & 180.4 & 45.53 \\
\hline 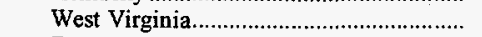 & 193.0 & 12,799 & .76 & 7.30 & 180.1 & 46.11 \\
\hline Total & 248.6 & 12,759 & .77 & 7.24 & 180.2 & 45.98 \\
\hline 1997 & & & & & & \\
\hline 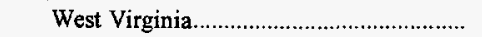 & 279.3 & 12,710 & .74 & 7.68 & 179.3 & 45.58 \\
\hline Total & 279.3 & 12,710 & .74 & 7.68 & 179.3 & 45.58 \\
\hline 1998 & & & & & & \\
\hline January - March & & & & & & \\
\hline West Virginia & 73.9 & 12,643 & .76 & 7.54 & 178.5 & 45.15 \\
\hline Total & 73.9 & 12,643 & .76 & 7.54 & 178.5 & 45.15 \\
\hline April - June & & & & & & \\
\hline 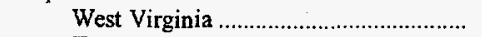 & 73.7 & 12,637 & .72 & 7.62 & 184.6 & 46.66 \\
\hline 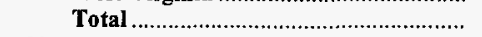 & 73.7 & 12,637 & .72 & 7.62 & 184.6 & 46.66 \\
\hline Year to Date & & & & & & \\
\hline West Virginia & 147.6 & 12,640 & .74 & 7.58 & 181.6 & 45.90 \\
\hline Total & 147.6 & 12,640 & .74 & 7.58 & 181.6 & 45.90 \\
\hline & & Company a & nt: New Eng & wer (NEES), & on Point & \\
\hline 1992 & & & & & & \\
\hline 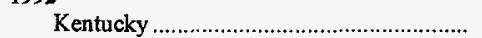 & 10.1 & 12,934 & 0.63 & 6.47 & 170.9 & 44.21 \\
\hline 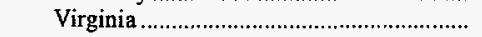 & 197.9 & 13,030 & 1.12 & 7.64 & 173.1 & 45.11 \\
\hline West Virginia & $2,209.9$ & 13,032 & 1.11 & 8.45 & 168.1 & 43.81 \\
\hline 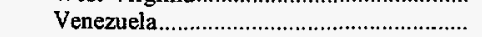 & 129.0 & 13,375 & .75 & 7.32 & 165.2 & 44.18 \\
\hline Total & $2,546.9$ & 13,049 & 1.09 & 8.32 & 168.3 & 43.94 \\
\hline 1993 & & & & & & \\
\hline 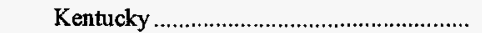 & 68.7 & 12,641 & .54 & 7.18 & 167.7 & 42.39 \\
\hline 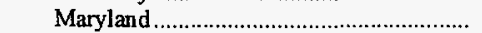 & 1.0 & 13,161 & 1.48 & 10.11 & 153.6 & 40.44 \\
\hline 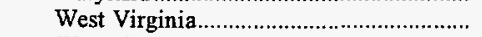 & $1,659.3$ & 12,985 & 1.05 & 8.54 & 167.5 & 43.51 \\
\hline 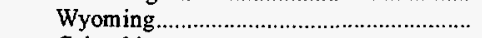 & 7.0 & 8,889 & .30 & 5.37 & 174.9 & 31.09 \\
\hline 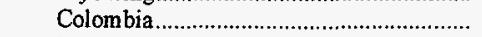 & 187.2 & 12,144 & .64 & 5.42 & 178.5 & 43.35 \\
\hline 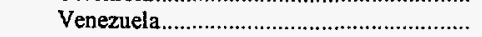 & 239.9 & 13,132 & .71 & 7.83 & 162.5 & 42.67 \\
\hline Total & $2,163.1$ & 12,905 & .96 & 8.14 & 167.9 & 43.33 \\
\hline
\end{tabular}

See footnotes at the end of Table A7. 
Table A7. Cost and Quality of All Coal Received at Electric Utility Plants that Import Coal by Origin, 1992-1998 (Continued)

\begin{tabular}{|c|c|c|c|c|c|c|}
\hline \multirow[b]{2}{*}{$\begin{array}{l}\text { Time Period and State } \\
\text { or Country of Origin }\end{array}$} & \multirow{2}{*}{$\begin{array}{l}\text { Quantity } \\
\text { (thousand } \\
\text { short tons) }\end{array}$} & \multicolumn{3}{|c|}{ Average Qualityl } & \multicolumn{2}{|c|}{ Average Cost Delivered } \\
\hline & & $\begin{array}{c}\text { Btu } \\
\text { per Pound }\end{array}$ & $\begin{array}{c}\text { Sulfur } \\
\text { Percent } \\
\text { by Weight }\end{array}$ & $\begin{array}{c}\text { Ash } \\
\text { Percent } \\
\text { by Weight }\end{array}$ & $\begin{array}{c}\text { Cents per } \\
\text { Million Btu }\end{array}$ & $\begin{array}{l}\text { Dollars per } \\
\text { Short Ton }\end{array}$ \\
\hline & \multicolumn{6}{|c|}{ Company and Plant: New England Power (NEES), Brayton Point } \\
\hline \multicolumn{7}{|l|}{1994} \\
\hline 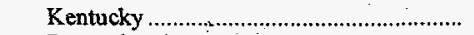 & 138.0 & 12,543 & 0.73 & 8.18 & 174.9 & 43.88 \\
\hline 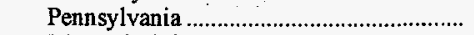 & 119.6 & 13,049 & 1.43 & 6.44 & 166.4 & 43.43 \\
\hline 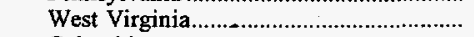 & $2,159.0$ & 12,823 & .98 & 8.61 & 170.6 & 43.75 \\
\hline 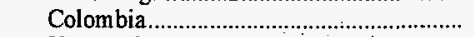 & 51.3 & 12,131 & .65 & 5.60 & 172.2 & 41.78 \\
\hline 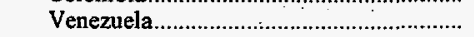 & 351.2 & 12,955 & .71 & 7.03 & 154.2 & 39.95 \\
\hline 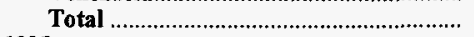 & $2,819.1$ & 12,822 & .95 & 8.24 & 168.6 & 43.24 \\
\hline \multicolumn{7}{|l|}{1995} \\
\hline 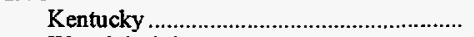 & 144.6 & 12,644 & .73 & 7.74 & 171.9 & 43.47 \\
\hline 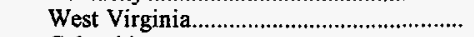 & $1,491.6$ & 12,687 & .71 & 9.56 & 171.3 & 43.46 \\
\hline 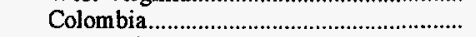 & 307.8 & 12,218 & .60 & 5.22 & 164.6 & 40.23 \\
\hline Venezuela & 510.6 & 12,788 & .69 & 7.03 & 160.0 & 40.92 \\
\hline 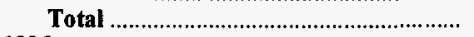 & $2,454.6$ & 12,647 & .69 & 8.38 & 168.1 & 42.53 \\
\hline \multicolumn{7}{|l|}{1996} \\
\hline Kentucky & 262.9 & 12,628 & .70 & 8.26 & 174.6 & 44.09 \\
\hline 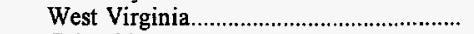 & $1,884.5$ & 12,538 & .70 & 10.16 & 172.9 & 43.37 \\
\hline 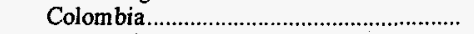 & 427.5 & 12,014 & .59 & 5.52 & 168.0 & 40.35 \\
\hline 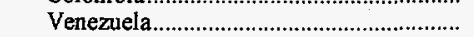 & 572.4 & 12,937 & .67 & 6.29 & 162.4 & 42.01 \\
\hline Total & $3,147.3$ & 12,547 & .68 & 8.67 & 170.5 & 42.77 \\
\hline \multicolumn{7}{|l|}{1997} \\
\hline (n) & 453.9 & 12,724 & .70 & 7.54 & 172.3 & 43.83 \\
\hline 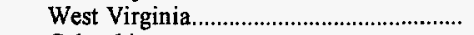 & $1,585.2$ & 12,526 & .70 & 10.62 & 168.8 & 42.29 \\
\hline 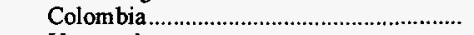 & 668.5 & 12,119 & .64 & 5.79 & 165.6 & 40.14 \\
\hline 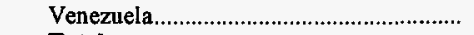 & 292.5 & 13,122 & .68 & 6.35 & 165.2 & 43.36 \\
\hline Total & $3,000.1$ & 12,524 & .69 & 8.66 & 168.3 & 42.15 \\
\hline \multicolumn{7}{|l|}{1998} \\
\hline \multicolumn{7}{|l|}{ January - March } \\
\hline 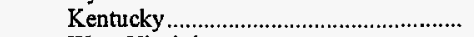 & 159.4 & 12,577 & .66 & 7.27 & 176.3 & 44.34 \\
\hline 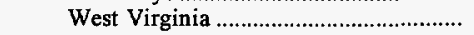 & 483.4 & 12,483 & .70 & 10.80 & 169.9 & 42.42 \\
\hline 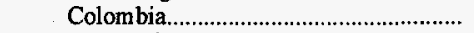 & 151.4 & 12,123 & .64 & 6.25 & 162.3 & 39.36 \\
\hline 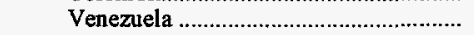 & 119.2 & 13,270 & .65 & 7.33 & 154.1 & 40.89 \\
\hline Total & 913.4 & 12,543 & .67 & 8.97 & 167.6 & 42.05 \\
\hline April - June & & & & & & \\
\hline Kentucky & 122.4 & 12,773 & .64 & 6.66 & 169.8 & 43.36 \\
\hline 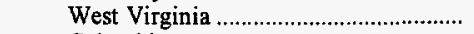 & 598.8 & 12,528 & .70 & 10.53 & 164.2 & 41.13 \\
\hline 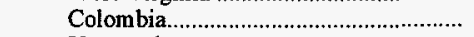 & 71.4 & 12,128 & .64 & 5.65 & 159.8 & 38.77 \\
\hline 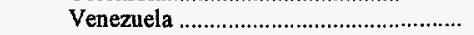 & 33.5 & 13,063 & .66 & 8.96 & 149.1 & 38.96 \\
\hline Total & 826.1 & 12,551 & .69 & 9.47 & 164.0 & 41.17 \\
\hline Year to Date & & & & & & \\
\hline 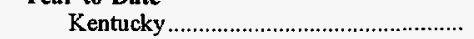 & 281.8 & 12,662 & .65 & 7.00 & 173.4 & 43.91 \\
\hline 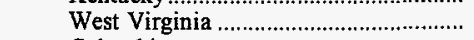 & $1,082.2$ & 12,508 & .70 & 10.65 & 166.7 & 41.71 \\
\hline 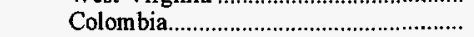 & 222.8 & 12,125 & .64 & 6.06 & 161.5 & 39.17 \\
\hline 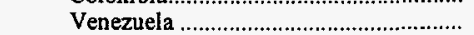 & 152.7 & 13,225 & .65 & 7.69 & 153.0 & 40.47 \\
\hline Total & $1,739.5$ & 12,547 & .68 & 9.21 & 165.9 & 41.63 \\
\hline & & Company a & nt: New Engl & wer (NEES), & Harbor & \\
\hline 1992 & & & & & & \\
\hline Pennsylvania & 40.2 & 13,193 & 1.26 & 6.80 & 162.3 & 42.82 \\
\hline 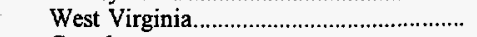 & 763.1 & 13,130 & 1.46 & 9.47 & 167.0 & 43.86 \\
\hline Canada & 32.8 & 13,569 & 1.40 & 3.82 & 174.9 & 47.46 \\
\hline 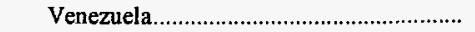 & 34.8 & 12,893 & .58 & 7.02 & 145.3 & 37.47 \\
\hline Total & 870.9 & 13,140 & 1.41 & 9.04 & 166.3 & 43.70 \\
\hline 1993 & & & & & & \\
\hline 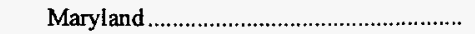 & 10.1 & 13,219 & 1.32 & 9.76 & 166.4 & 43.99 \\
\hline 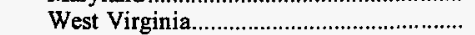 & 532.9 & 13,013 & 1.29 & 9.62 & 167.2 & 43.51 \\
\hline 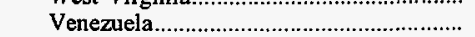 & 236.2 & 12,921 & .57 & 6.65 & 162.5 & 41.99 \\
\hline Total & 779.2 & 12,987 & 1.07 & 8.72 & 165.8 & 43.05 \\
\hline 1994 & & & & & & \\
\hline 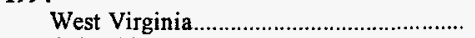 & 80.0 & 12,958 & .77 & 8.71 & 177.5 & 45.99 \\
\hline Colombia & 84.2 & 12,017 & .57 & 6.07 & 159.9 & 38.44 \\
\hline 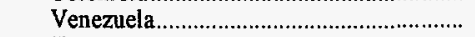 & 565.5 & 12,678 & .64 & 6.49 & 159.6 & 40.47 \\
\hline 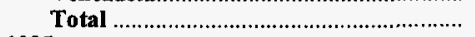 & 729.7 & 12,632 & .65 & 6.69 & 161.6 & 40.84 \\
\hline 1995 & & & & & & \\
\hline 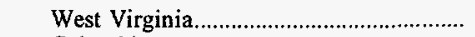 & 117.6 & 12,643 & .68 & 8.94 & 183.5 & 46.41 \\
\hline Colombia & 250.1 & 12,166 & .60 & 5.26 & 147.9 & 35.99 \\
\hline 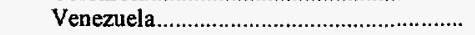 & 393.1 & 12,846 & .65 & 6.34 & 162.4 & 41.72 \\
\hline 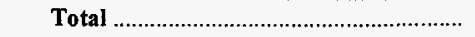 & 760.8 & 12,591 & .64 & 6.39 & 161.1 & 40.56 \\
\hline
\end{tabular}

See footnotes at the end of Table A7. 
Table A7. Cost and Quality of All Coal Received at Electric Utility Plants that Import Coal by Origin, 1992-1998 (Continued)

\begin{tabular}{|c|c|c|c|c|c|c|}
\hline \multirow[b]{2}{*}{$\begin{array}{l}\text { Time Period and State } \\
\text { or Country of Origin }\end{array}$} & \multirow[b]{2}{*}{$\begin{array}{l}\text { Quantity } \\
\text { (thousand } \\
\text { short tons) }\end{array}$} & \multicolumn{3}{|c|}{ Average Quality 1} & \multicolumn{2}{|c|}{ Average Cost Delivered } \\
\hline & & $\begin{array}{c}\text { Btu } \\
\text { per Pound }\end{array}$ & $\begin{array}{c}\text { Sulfur } \\
\text { Percent } \\
\text { by Weight }\end{array}$ & $\begin{array}{c}\text { Ash } \\
\text { Percent } \\
\text { by Weight }\end{array}$ & $\begin{array}{l}\text { Cents per } \\
\text { Million Btu }\end{array}$ & $\begin{array}{l}\text { Dollars per } \\
\text { Short Ton }\end{array}$ \\
\hline & \multicolumn{6}{|c|}{ Company and Plant: New England Power (NEES), Salem Harbor } \\
\hline \multicolumn{7}{|l|}{1996} \\
\hline Kentucky & 2.6 & 12,282 & 0.63 & 8.35 & 155.3 & 38.16 \\
\hline 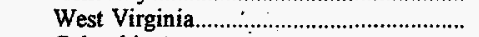 & 157.2 & 12,727 & .70 & 9.20 & 176.6 & 44.95 \\
\hline 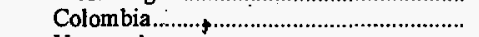 & 202.9 & 12,069 & .57 & 5.77 & 148.6 & 35.86 \\
\hline 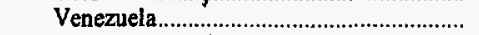 & 563.1 & 12,849 & .70 & 6.17 & 155.5 & 39.96 \\
\hline Total & 925.8 & 12,656 & .67 & 6.60 & 157.6 & 39.90 \\
\hline \multicolumn{7}{|l|}{1997} \\
\hline 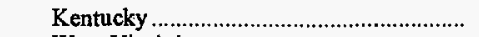 & 136.2 & 12,613 & .66 & 7.78 & 176.6 & 44.55 \\
\hline 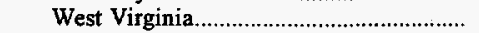 & 181.1 & 12,500 & .69 & 10.75 & 165.8 & 41.46 \\
\hline 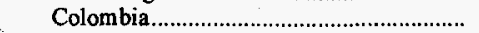 & 409.1 & 12,101 & .63 & 6.17 & 167.2 & 40.46 \\
\hline 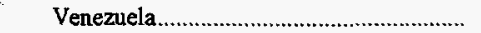 & 90.4 & 12,934 & .69 & 5.82 & 156.8 & 40.58 \\
\hline Total & 816.8 & 12,367 & .66 & 7.41 & 167.3 & 41.37 \\
\hline \multicolumn{7}{|l|}{1998} \\
\hline \multicolumn{7}{|l|}{ January - March } \\
\hline West Virginia & 40.9 & 12,447 & .74 & 11.20 & 167.5 & 41.71 \\
\hline 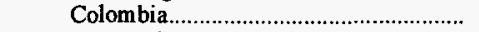 & 76.7 & 12,117 & .57 & 5.91 & 189.1 & 45.82 \\
\hline 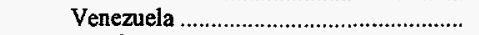 & 100.9 & 13,000 & .70 & 5.59 & 156.8 & 40.77 \\
\hline Total & $\mathbf{2 1 8 . 5}$ & 12,586 & .66 & 6.75 & 169.7 & $\mathbf{4 2 . 7 2}$ \\
\hline \multicolumn{7}{|l|}{ April - June } \\
\hline West Virginia & 40.2 & 12,240 & .70 & 11.62 & 159.4 & 39.01 \\
\hline 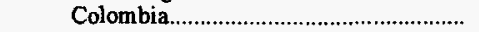 & 87.5 & 12,120 & .60 & 5.16 & 158.7 & 38.46 \\
\hline 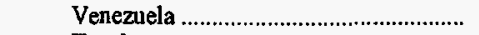 & 53.8 & 13,086 & .70 & 5.05 & 146.8 & 38.42 \\
\hline Total & 181.5 & 12,433 & .65 & 6.56 & 155.1 & 38.57 \\
\hline \multicolumn{7}{|l|}{ Year to Date } \\
\hline 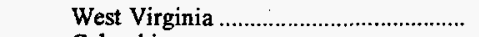 & 81.1 & 12,344 & .72 & 11.41 & 163.5 & 40.37 \\
\hline 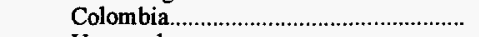 & 164.2 & 12,119 & .59 & 5.51 & 172.9 & 41.90 \\
\hline 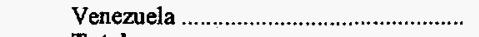 & 154.7 & 13,030 & .70 & 5.40 & 153.3 & 39.96 \\
\hline 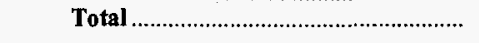 & 400.0 & 12,517 & .66 & 6.66 & 163.1 & 40.84 \\
\hline & & & and Plant: & dison, Burge & & \\
\hline 1992 & & & & & & \\
\hline Kentucky & 41.4 & 12,143 & 0.84 & 10.37 & 130.5 & 31.69 \\
\hline Ohio & 963.7 & 12,135 & 3.62 & 11.27 & 104.4 & 25.35 \\
\hline 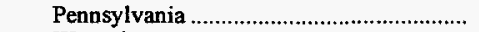 & 128.2 & 12,070 & 2.83 & 11.73 & 129.8 & 31.32 \\
\hline 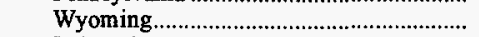 & 61.3 & 8,449 & .35 & 5.48 & 120.1 & 20.29 \\
\hline 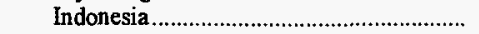 & 13.1 & 9,587 & .14 & 1.20 & 166.9 & 32.00 \\
\hline 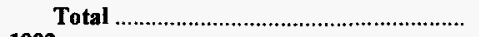 & $1,207.7$ & 11,913 & 3.24 & 10.88 & 109.2 & 26.01 \\
\hline 1993 & & & & & & \\
\hline 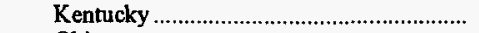 & 6.1 & 12,223 & .88 & 10.70 & 110.4 & 26.99 \\
\hline Ohio & $1,151.5$ & 12,135 & 3.57 & 11.37 & 102.2 & 24.81 \\
\hline Pennsylvania & 99.9 & 11,842 & 3.41 & 11.82 & 92.0 & 21.79 \\
\hline 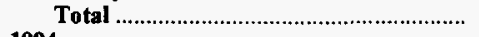 & $1,257.5$ & 12,113 & 3.55 & 11.41 & 101.5 & $\mathbf{2 4 . 5 8}$ \\
\hline 1994 & & & & & & \\
\hline Ohio & 937.8 & 12,266 & 3.58 & 10.63 & 99.0 & 24.28 \\
\hline 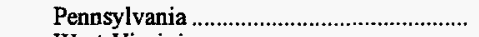 & 63.6 & 11,942 & 2.80 & 11.52 & 105.5 & 25.20 \\
\hline 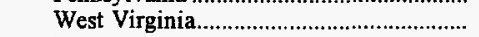 & 1.5 & 11,112 & 4.48 & 17.60 & 112.7 & 25.05 \\
\hline Total & $1,002.9$ & 12,244 & $\mathbf{3 . 5 3}$ & 10.69 & 99.4 & 24.34 \\
\hline 1995 & & & & & & \\
\hline 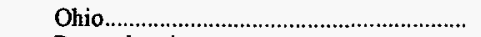 & 225.7 & 12,444 & 3.78 & 10.17 & 95.2 & 23.68 \\
\hline 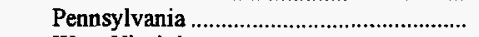 & 179.8 & 12,635 & 2.41 & 10.26 & 93.0 & 23.49 \\
\hline 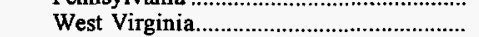 & 158.1 & 12,320 & 2.49 & 11.49 & 90.5 & 22.29 \\
\hline 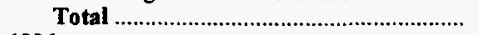 & 563.6 & 12,470 & 2.98 & 10.57 & 93.1 & 23.23 \\
\hline 1996 & & & & & & \\
\hline Ohio & 2.3 & 11,043 & 2.57 & 10.80 & 72.6 & 16.03 \\
\hline 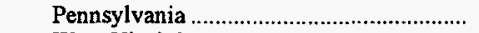 & 33.4 & 11,981 & 3.38 & 11.11 & 77.5 & 18.56 \\
\hline West Virginia & 942.8 & 12,323 & 3.69 & 9.65 & 81.9 & 20.18 \\
\hline Total & 978.5 & 12,308 & 3.68 & 9.70 & 81.7 & 20.11 \\
\hline 1997 & & & & & & \\
\hline 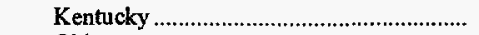 & 30.3 & 11,362 & .70 & 12.77 & 108.4 & 24.63 \\
\hline Ohio & 201.5 & 11,728 & 3.06 & 12.49 & 92.6 & 21.73 \\
\hline 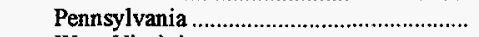 & 199.4 & 10,651 & 2.14 & 20.27 & 89.6 & 19.09 \\
\hline 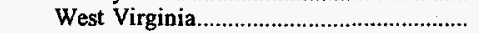 & 420.0 & 11,619 & 2.16 & 15.77 & 88.4 & 20.55 \\
\hline Total & 851.2 & 11,409 & 2.32 & 15.94 & 90.4 & 20.63 \\
\hline 1998 & & & & & & \\
\hline January - March & & & & & & \\
\hline Ohio & 63.0 & 11,992 & 3.02 & 11.70 & 98.5 & 23.62 \\
\hline 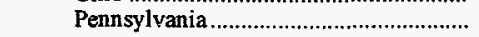 & 70.1 & 13,184 & 2.22 & 7.61 & 104.8 & 27.65 \\
\hline
\end{tabular}

See footnotes at the end of Table A7. 
Table A7. Cost and Quality of All Coal Received at Electric Utility Plants that Import Coal by Origin, 1992-1998 (Continued)

\begin{tabular}{|c|c|c|c|c|c|c|}
\hline \multirow[b]{2}{*}{$\begin{array}{l}\text { Time Period and State } \\
\text { or Country of Origin }\end{array}$} & \multirow{2}{*}{$\begin{array}{l}\text { Quantity } \\
\text { (thousand } \\
\text { short tons) }\end{array}$} & \multicolumn{3}{|c|}{ Average Quality 1} & \multicolumn{2}{|c|}{ Average Cost Delivered } \\
\hline & & $\begin{array}{l}\text { Btu } \\
\text { per Pound }\end{array}$ & $\begin{array}{c}\text { Sulfur } \\
\text { Percent } \\
\text { by Weight }\end{array}$ & $\begin{array}{c}\text { Ash } \\
\text { Percent } \\
\text { by Weight }\end{array}$ & $\begin{array}{l}\text { Cents per } \\
\text { Million Btu }\end{array}$ & $\begin{array}{l}\text { Dollars per } \\
\text { Short Ton }\end{array}$ \\
\hline & \multicolumn{6}{|c|}{ Company and Plant: Ohio Edison, Burger Plant } \\
\hline \multicolumn{7}{|l|}{1998} \\
\hline West Virginia & 50.5 & 11,707 & 0.85 & 15.21 & 107.2 & 25.09 \\
\hline $\begin{array}{l}\text { Total } \\
\text { April - June }\end{array}$ & 183.6 & 12,369 & 2.11 & 11.10 & 103.3 & $\mathbf{2 5 . 5 6}$ \\
\hline 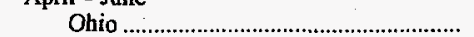 & 82.0 & 12,366 & 3.51 & 10.23 & 88.4 & 21.86 \\
\hline 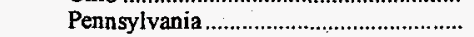 & 70.6 & 13,195 & 2.05 & 7.77 & 102.7 & 27.11 \\
\hline 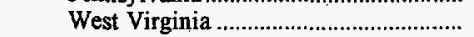 & 21.9 & 11,673 & 3.38 & 13.24 & 88.2 & 20.60 \\
\hline Total & 174.5 & 12,615 & 2.90 & 9.61 & 94.4 & 23.82 \\
\hline \multicolumn{7}{|l|}{ Year to Date } \\
\hline Ohio & 145.0 & 12,203 & 3.29 & 10.87 & 92.7 & 22.62 \\
\hline 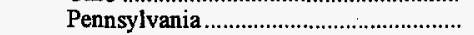 & 140.7 & 13,190 & 2.14 & 7.69 & 103.8 & 27.38 \\
\hline \multirow{3}{*}{ 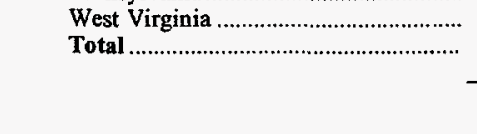 } & 72.4 & 11,697 & 1.61 & 14.61 & 101.4 & 23.73 \\
\hline & 358.1 & 12,489 & 2.50 & 10.38 & 98.9 & 24.71 \\
\hline & \multicolumn{6}{|c|}{ Company and Plant: Public Serv Co of Indiana, Gallagher } \\
\hline \multicolumn{7}{|l|}{1992} \\
\hline 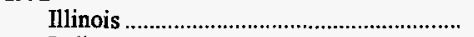 & 51.3 & 10,841 & 3.41 & 7.97 & 185.5 & 40.21 \\
\hline Indiana & 826.6 & 10,901 & 2.26 & 8.78 & 142.3 & 31.01 \\
\hline 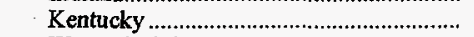 & 120.1 & 11,907 & 1.49 & 9.48 & 114.9 & 27.37 \\
\hline 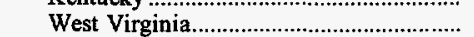 & 146.5 & 12,744 & .77 & 8.82 & 115.4 & 29.41 \\
\hline 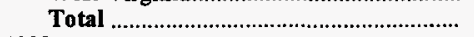 & $1,144.5$ & 11,240 & 2.04 & 8.82 & 137.2 & 30.84 \\
\hline \multicolumn{7}{|l|}{1993} \\
\hline 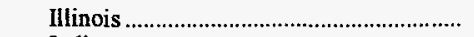 & 11.8 & 11,792 & 1.52 & 6.70 & 102.7 & 24.23 \\
\hline Indiana & 466.6 & 10,994 & 2.12 & 8.81 & 137.1 & 30.14 \\
\hline 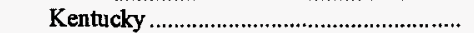 & 58.3 & 11,923 & 1.70 & 11.59 & 122.2 & 29.13 \\
\hline 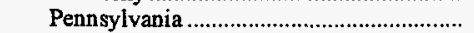 & 173.7 & 13,213 & 2.53 & 7.34 & 132.3 & 34.97 \\
\hline Indonesia & 11.1 & 9,242 & .13 & 1.35 & 104.8 & 19.38 \\
\hline 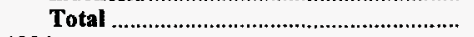 & 721.5 & 11,589 & 2.14 & 8.53 & 133.6 & 30.96 \\
\hline 1994 & & & & & & \\
\hline Illinois & 362.8 & 11,905 & 1.53 & 7.19 & 130.0 & 30.96 \\
\hline 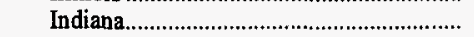 & 326.8 & 11,062 & 1.82 & 8.77 & 121.5 & 26.88 \\
\hline 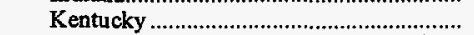 & 304.0 & 11,849 & 1.73 & 11.81 & 132.6 & 31.42 \\
\hline 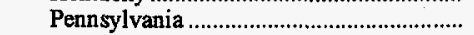 & 492.1 & 13,237 & 2.29 & 7.59 & 112.9 & 29.89 \\
\hline 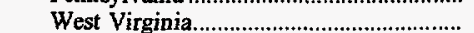 & 31.9 & 12,451 & 1.30 & 10.41 & 121.3 & 30.20 \\
\hline Total & $1,517.6$ & 12,155 & 1.88 & 8.65 & 122.6 & 29.81 \\
\hline 1995 & & & & & & \\
\hline 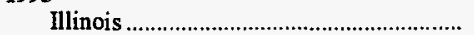 & 445.0 & 11,913 & 1.43 & 7.05 & 123.4 & 29.41 \\
\hline Indiana & 133.3 & 11,064 & 1.31 & 9.65 & 116.2 & 25.72 \\
\hline Pennsylvania & 547.4 & 13,131 & 2.35 & 7.97 & 102.4 & 26.90 \\
\hline Total & $1,125.7$ & 12,405 & 1.86 & 7.80 & 111.9 & 27.75 \\
\hline 1996 & & & & & & \\
\hline 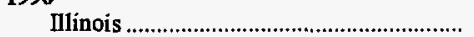 & 376.7 & 11,970 & 1.48 & 6.65 & 115.5 & 27.66 \\
\hline Indiana & 18.1 & 11,256 & 1.58 & 7.15 & 97.7 & 21.99 \\
\hline Kentucky & 73.6 & 12,343 & 2.15 & 9.89 & 108.9 & 26.89 \\
\hline 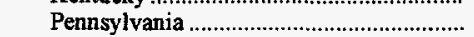 & 531.6 & 13,043 & 2.35 & 8.06 & 108.7 & 28.35 \\
\hline Total & $1,000.0$ & 12,555 & 1.99 & 7.64 & 111.0 & 27.87 \\
\hline 1997 & & & & & & \\
\hline 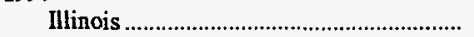 & 108.0 & 11,889 & 1.09 & 6.71 & 124.0 & 29.49 \\
\hline Indiana. & 5.2 & 10,949 & 2.25 & 8.76 & 107.6 & 23.56 \\
\hline 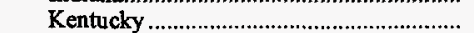 & 17.2 & 11,889 & 2.27 & 10.52 & 116.2 & 27.64 \\
\hline 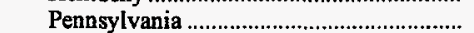 & 647.0 & 13,052 & 2.30 & 8.01 & 104.1 & 27.18 \\
\hline 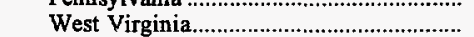 & 207.7 & 13,202 & 2.13 & 7.14 & 106.3 & 28.06 \\
\hline Total & 985.1 & 12,924 & 2.13 & 7.73 & 106.8 & 27.61 \\
\hline 1998 & & & & & & \\
\hline January - March & & & & & & \\
\hline 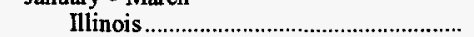 & 38.2 & 11,958 & .80 & 5.15 & 125.5 & 30.01 \\
\hline 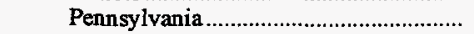 & 162.2 & 12,990 & 2.23 & 7.92 & 106.8 & 27.74 \\
\hline 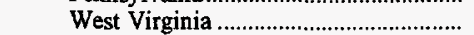 & 157.2 & 13,217 & 2.30 & 6.74 & 107.1 & 28.31 \\
\hline Total & 357.6 & 12,980 & 2.11 & 7.10 & 108.8 & 28.23 \\
\hline April - June & & & & & & \\
\hline Indiana & 1.8 & 11,343 & 2.20 & 8.20 & 97.7 & 22.16 \\
\hline Pennsylvania & 120.9 & 13,000 & 2.09 & 7.42 & 106.5 & 27.68 \\
\hline 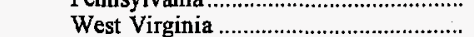 & 64.6 & 13,033 & 2.22 & 7.77 & 106.9 & 27.86 \\
\hline 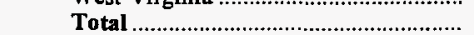 & 187.3 & 12,995 & 2.14 & 7.55 & 106.5 & 27.69 \\
\hline
\end{tabular}

See footnotes at the end of Table A7. 
Table A7. Cost and Quality of All Coal Received at Electric Utility Plants that Import Coal by Origin, 1992-1998 (Continued)

\begin{tabular}{|c|c|c|c|c|c|c|}
\hline \multirow[b]{2}{*}{$\begin{array}{l}\text { Time Period and State } \\
\text { or Country of Origin }\end{array}$} & \multirow[b]{2}{*}{$\begin{array}{l}\text { Quantity } \\
\text { (thousand } \\
\text { short tons) }\end{array}$} & \multicolumn{3}{|c|}{ Average Quality 1} & \multicolumn{2}{|c|}{ Average Cost Delivered } \\
\hline & & $\begin{array}{c}\text { Btu } \\
\text { per Pound }\end{array}$ & $\begin{array}{c}\text { Sulfur } \\
\text { Percent } \\
\text { by Weight }\end{array}$ & $\begin{array}{c}\text { Ash } \\
\text { Percent } \\
\text { by Weight }\end{array}$ & $\begin{array}{l}\text { Cents per } \\
\text { Million Btu }\end{array}$ & $\begin{array}{l}\text { Dollars per } \\
\text { Short Ton }\end{array}$ \\
\hline & \multicolumn{6}{|c|}{ Company and Plant: Public Serv Co of Indiana, Gallagher } \\
\hline \multicolumn{6}{|l|}{1998} & \\
\hline Illinois.... & 38.2 & 11,958 & 0.80 & 5.15 & 125.5 & 30.01 \\
\hline Indiana & 1.8 & 11,343 & 2.20 & 8.20 & 97.7 & 22.16 \\
\hline Pennsylyania. & 283.1 & 12,994 & 2.17 & 7.70 & 106.6 & 27.71 \\
\hline West Virginia & 221.8 & 13,164 & 2.28 & 7.04 & 107.0 & 28.18 \\
\hline \multirow[t]{2}{*}{ Total } & 544.9 & 12,985 & 2.12 & 7.26 & 108.0 & 28.05 \\
\hline & \multicolumn{6}{|c|}{ Company and Plant: Public Serv Co of New Hampshire, Merrimack } \\
\hline \multicolumn{7}{|l|}{1992} \\
\hline 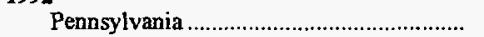 & 671.5 & 13,266 & 1.57 & 6.30 & 171.5 & 45.50 \\
\hline 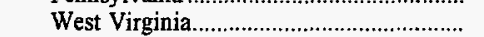 & 331.8 & 13,416 & 2.27 & 6.94 & 161.4 & 43.30 \\
\hline Total & $1,003.3$ & 13,316 & 1.80 & 6.51 & 168.1 & 44.77 \\
\hline \multicolumn{7}{|l|}{1993} \\
\hline 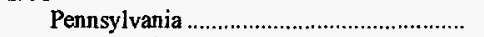 & 661.6 & 13,240 & 1.63 & 6.42 & 165.9 & 43.92 \\
\hline 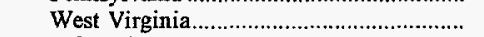 & 388.6 & 13,225 & 2.27 & 7.59 & 155.3 & 41.07 \\
\hline Indonesia & 21.2 & 12,620 & .49 & 3.80 & 186.5 & 47.07 \\
\hline 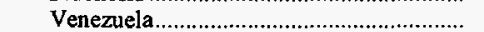 & 24.9 & 12,920 & .58 & 6.00 & 163.2 & 42.17 \\
\hline Total & $1,096.3$ & 13,216 & 1.81 & 6.77 & 162.4 & 42.93 \\
\hline \multicolumn{7}{|l|}{1994} \\
\hline 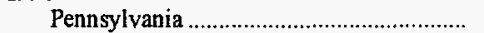 & 706.9 & 13,176 & 1.57 & 6.61 & 156.5 & 41.25 \\
\hline 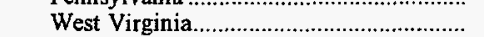 & 272.1 & 13,253 & 2.34 & 7.50 & 147.8 & 39.17 \\
\hline Total & 979.0 & 13,197 & 1.78 & 6.86 & 154.1 & 40.67 \\
\hline \multicolumn{7}{|l|}{1995} \\
\hline 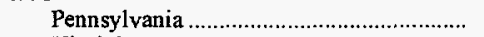 & 759.3 & 13,203 & 1.49 & 6.90 & 161.1 & 42.53 \\
\hline 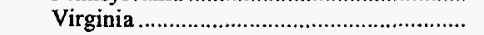 & 19.1 & 13,910 & .68 & 7.00 & 203.5 & 56.61 \\
\hline 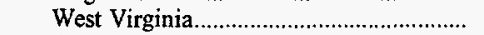 & 223.3 & 13,366 & 2.29 & 6.28 & 141.7 & 37.89 \\
\hline 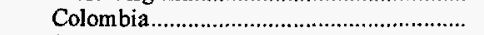 & 11.5 & 11,578 & .53 & 3.80 & 192.9 & 44.67 \\
\hline 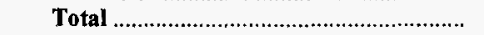 & $1,013.2$ & 13,234 & 1.64 & 6.73 & 157.9 & 41.80 \\
\hline 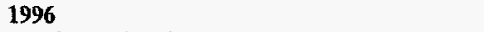 & & & & & & \\
\hline 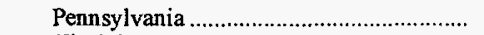 & 715.4 & 13,233 & 1.53 & 6.72 & 162.3 & 42.95 \\
\hline Virginia & 16.0 & 14,085 & .74 & 5.83 & 199.8 & 56.30 \\
\hline 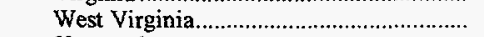 & 278.0 & 13,320 & 2.34 & 6.64 & 147.0 & 39.15 \\
\hline 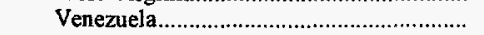 & 39.9 & 12,370 & .39 & 3.70 & 213.2 & 52.75 \\
\hline Total & $1,049.3$ & 13,236 & 1.69 & 6.57 & 160.6 & 42.52 \\
\hline 1997 & & & & & & \\
\hline 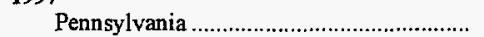 & 830.3 & 13,221 & 1.46 & 6.97 & 166.2 & 43.95 \\
\hline Virginia & 9.9 & 13,759 & 1.46 & 5.37 & 212.5 & 58.47 \\
\hline 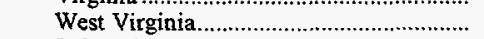 & 312.8 & 13,325 & 2.06 & 6.86 & 154.2 & 41.10 \\
\hline Indonesia & 40.8 & 12,300 & .49 & 4.50 & 190.7 & 46.92 \\
\hline Total & $1,193.7$ & 13,221 & 1.58 & 6.84 & 164.2 & $\mathbf{4 3 . 4 3}$ \\
\hline 1998 & & & & & & \\
\hline January - March & & & & & & \\
\hline Pennsylvania & 187.4 & 13,129 & 1.44 & 7.30 & 165.0 & 43.33 \\
\hline 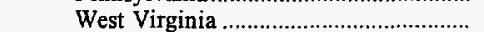 & 73.6 & 13,266 & 2.47 & 7.25 & 160.1 & 42.47 \\
\hline 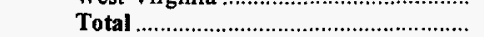 & 261.0 & 13,168 & 1.73 & 7.29 & 163.6 & 43.09 \\
\hline April - June & & & & & & \\
\hline 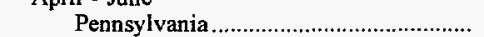 & 143.4 & 13,159 & 1.46 & 6.88 & 166.9 & 43,92 \\
\hline West Virginia & 43.8 & 13,296 & 2.29 & 6.69 & 160.0 & 42.54 \\
\hline Total & 187.1 & 13,191 & 1.66 & 6.84 & 165.2 & 43.59 \\
\hline Year to Date & & & & & & \\
\hline 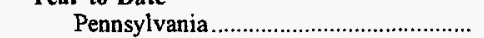 & 330.7 & 13,142 & 1.45 & 7.12 & 165.8 & 43.59 \\
\hline West Virginia & 117.4 & 13,277 & 2.40 & 7.04 & 160.0 & 42.50 \\
\hline Total & 448.1 & 13,177 & 1.70 & 7.10 & 164.3 & 43.30 \\
\hline & & Company : & Int: Public Se & of New Ham & Schiller & \\
\hline 1992 & & & & & & \\
\hline 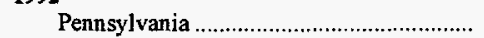 & 8.3 & 13,080 & 1.46 & 6.25 & 173.0 & 45.26 \\
\hline West Virginia & 131.9 & 13,252 & .77 & 6.62 & 175.2 & 46.44 \\
\hline 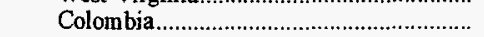 & 48.4 & 12,428 & .61 & 6.31 & 157.2 & 39.08 \\
\hline 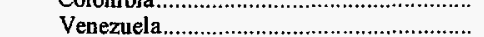 & $\begin{array}{l}40.4 \\
34.3\end{array}$ & 12,881 & .58 & 6.76 & 168.0 & 43.29 \\
\hline Total & 222.9 & 13,010 & .73 & 6.56 & 170.3 & 44.31 \\
\hline 1993 & & & & & & \\
\hline West Virginia & 57.6 & 13,238 & .75 & 7.40 & 171.7 & 45.45 \\
\hline 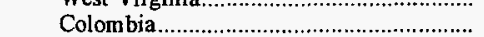 & 52.1 & 12,861 & .64 & 7.49 & 150.0 & 38.59 \\
\hline 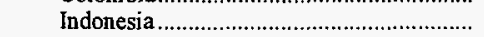 & 16.0 & 12,620 & .49 & 3.80 & 161.3 & 40.71 \\
\hline
\end{tabular}

See footnotes at the end of Table A7. 
Table A7. Cost and Quality of All Coal Received at Electric Utility Plants that Import Coal by Origin, 1992-1998 (Continued)

\begin{tabular}{|c|c|c|c|c|c|c|}
\hline \multirow[b]{2}{*}{$\begin{array}{l}\text { Time Period and State } \\
\text { or Country of Origin }\end{array}$} & \multirow{2}{*}{$\begin{array}{l}\text { Quantity } \\
\text { (thousand } \\
\text { short tons) }\end{array}$} & \multicolumn{3}{|c|}{ Average Quality 1} & \multicolumn{2}{|c|}{ Average Cost Delivered } \\
\hline & & $\begin{array}{c}\text { Btu } \\
\text { per Pound }\end{array}$ & $\begin{array}{c}\text { Sulfur } \\
\text { Percent } \\
\text { by Weight }\end{array}$ & $\begin{array}{c}\text { Ash } \\
\text { Percent } \\
\text { by Weight }\end{array}$ & $\begin{array}{l}\text { Cents per } \\
\text { Million Btu }\end{array}$ & $\begin{array}{l}\text { Dollars per } \\
\text { Short Ton }\end{array}$ \\
\hline & \multicolumn{6}{|c|}{ Company and Plant: Public Serv Co of New Hampshire, Schiller } \\
\hline \multicolumn{7}{|l|}{1993} \\
\hline 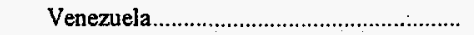 & 84.3 & 12,972 & 0.58 & 6.08 & 138.6 & 35.95 \\
\hline 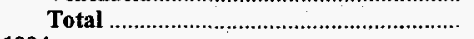 & 210.1 & 12,991 & .63 & 6.62 & 152.3 & 39.58 \\
\hline \multicolumn{7}{|l|}{1994} \\
\hline Colombia. & 163.3 & 12,505 & .62 & 5.55 & 135.5 & 33.89 \\
\hline 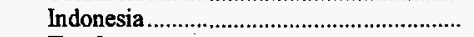 & 113.0 & 12,360 & .53 & 3.58 & 158.7 & 39.23 \\
\hline 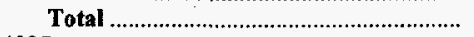 & 276.3 & 12,446 & .58 & 4.74 & 144.9 & 36.07 \\
\hline \multicolumn{7}{|l|}{1995} \\
\hline West Virginia & 74.1 & 12,997 & .80 & 8.76 & 164.3 & 42.72 \\
\hline 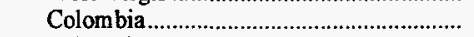 & 122.9 & 12,733 & .62 & 6.70 & 160.0 & 40.73 \\
\hline 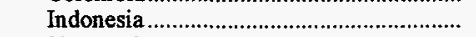 & 79.7 & 12,300 & .52 & 4.56 & 167.8 & 41.28 \\
\hline 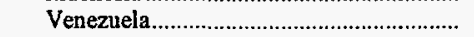 & 82.4 & 13,044 & .71 & 7.24 & 156.5 & 40.84 \\
\hline Total & 359.1 & 12,762 & .66 & 6.77 & 161.8 & 41.29 \\
\hline \multicolumn{7}{|l|}{1996} \\
\hline 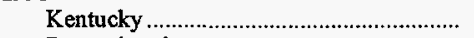 & 14.5 & 12,830 & .90 & 8.40 & 193.7 & 49.70 \\
\hline 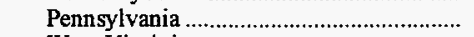 & 44.0 & 12,708 & 1.53 & 13.21 & 155.8 & 39.59 \\
\hline 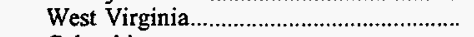 & 101.3 & 12,988 & 1.38 & 9.41 & 157.6 & 40.95 \\
\hline 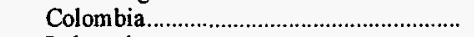 & 32.3 & 12,169 & .66 & 5.68 & 161.9 & 39.41 \\
\hline 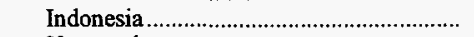 & 25.9 & 12,412 & .72 & 8.20 & 161.9 & 40.19 \\
\hline 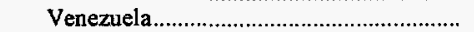 & 56.1 & 13,061 & .67 & 6.04 & 159.8 & 41.75 \\
\hline Total & 274.2 & 12,799 & 1.09 & 8.72 & 160.6 & 41.11 \\
\hline \multicolumn{7}{|l|}{1997} \\
\hline Pennsylvania & 29.5 & 13,002 & 1.75 & 8.00 & 159.2 & 41.40 \\
\hline 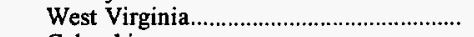 & 140.0 & 12,969 & 1.41 & 8.20 & 160.0 & 41.51 \\
\hline Colombia & 35.4 & 13,231 & .63 & 6.70 & 160.1 & 42.37 \\
\hline 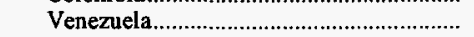 & 229.0 & 12,217 & .67 & 6.13 & 160.7 & 39.27 \\
\hline Total & 433.9 & 12,596 & .98 & 6.97 & 160.3 & 40.39 \\
\hline \multicolumn{7}{|l|}{1998} \\
\hline January - March & & & & & & \\
\hline 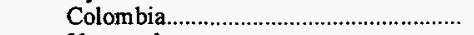 & 34.7 & 13,188 & .64 & 5.50 & 172.8 & 45.58 \\
\hline 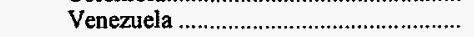 & 73.9 & 13,161 & .65 & 5.20 & 148.9 & 39.20 \\
\hline Total & 108.6 & 13,170 & .65 & 5.29 & 156.6 & 41.24 \\
\hline April - June & & & & & & \\
\hline 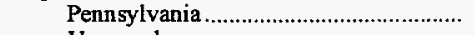 & 14.7 & 13,018 & 1.40 & 6.90 & 159.8 & 41.60 \\
\hline 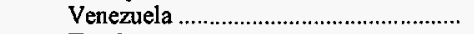 & 73.8 & 12,796 & .68 & 6.15 & 145.8 & 37.30 \\
\hline 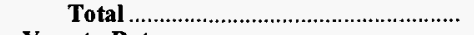 & 88.5 & 12,833 & .80 & 6.28 & 148.1 & 38.01 \\
\hline Year to Date & & & & & & \\
\hline Pennsylvania & 14.7 & 13,018 & 1.40 & 6.90 & 159.8 & 41.60 \\
\hline 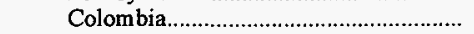 & 34.7 & 13,188 & .64 & 5.50 & 172.8 & 45.58 \\
\hline 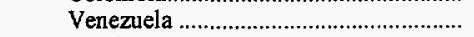 & 147.7 & 12,979 & .67 & 5.67 & 147.4 & 38.25 \\
\hline Total & 197.1 & 13,018 & .72 & 5.73 & 152.8 & 39.79 \\
\hline & & Compan & 'lant: Public & ectric \& Gas & lercer & \\
\hline 1992 & & & & & & \\
\hline Virginia & 805.6 & 14,185 & 0.83 & 4.40 & 175.9 & 49.90 \\
\hline 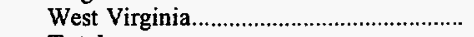 & 35.3 & 13,875 & .64 & 5.71 & 170.8 & 47.40 \\
\hline Total & 840.9 & 14,172 & .82 & 4.46 & 175.7 & 49.80 \\
\hline 1993 & & & & & & \\
\hline Virginia & 695.9 & 14,123 & .80 & 4.54 & 179.6 & 50.73 \\
\hline 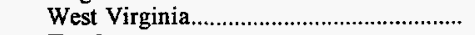 & 7.2 & 13,648 & .64 & 5.91 & 174.6 & 47.65 \\
\hline 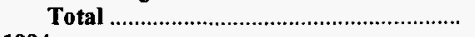 & 703.1 & 14,118 & .80 & 4.56 & 179.6 & 50.70 \\
\hline 1994 & & & & & & \\
\hline Virginia & 687.7 & 14,046 & .79 & 4.73 & 179.8 & 50.50 \\
\hline 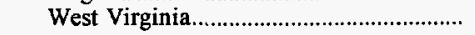 & .7 & 13,381 & .66 & 6.60 & 174.6 & 46.73 \\
\hline Total & 688.4 & 14,045 & .79 & 4.73 & 179.8 & $\mathbf{5 0 . 5 0}$ \\
\hline 1995 & & & & & & \\
\hline Virginia & 688.8 & 13,985 & .77 & 4.89 & 175.3 & 49.03 \\
\hline 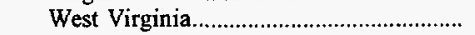 & .1 & 13,529 & .70 & 6.70 & 145.3 & 39.32 \\
\hline 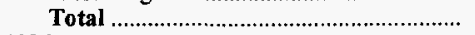 & 688.9 & 13,985 & .77 & 4.89 & 175.3 & 49.03 \\
\hline 1996 & & & & & & \\
\hline Virginia & 635.8 & 13,897 & .77 & 5.36 & 181.6 & 50.46 \\
\hline 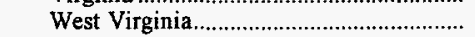 & .8 & 12,705 & .97 & 8.20 & 172.9 & 43.93 \\
\hline Total & 636.6 & 13,896 & .77 & 5.36 & 181.5 & 50.45 \\
\hline 1997 & & & & & & \\
\hline 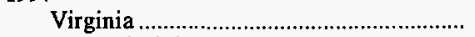 & 880.3 & 13,634 & .78 & 5.85 & 174.7 & 47.64 \\
\hline 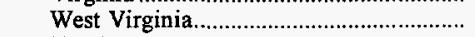 & .8 & 12,514 & .92 & 8.60 & 164.6 & 41.20 \\
\hline Total & 881.1 & 13,633 & .78 & 5.85 & 174.7 & 47.64 \\
\hline
\end{tabular}

See footnotes at the end of Table A7. 
Table A7. Cost and Quality of All Coal Received at Electric Utility Plants that Import Coal by Origin, 1992-1998 (Continued)

\begin{tabular}{|c|c|c|c|c|c|c|}
\hline \multirow[b]{2}{*}{$\begin{array}{l}\text { Time Period and State } \\
\text { or Country of Origin }\end{array}$} & \multirow[b]{2}{*}{$\begin{array}{l}\text { Quantity } \\
\text { (thousand } \\
\text { short tons) }\end{array}$} & \multicolumn{3}{|c|}{ Average Qualityl } & \multicolumn{2}{|c|}{ Average Cost Delivered } \\
\hline & & $\begin{array}{c}\text { Btu } \\
\text { per Pound }\end{array}$ & $\begin{array}{c}\text { Sulfur } \\
\text { Percent } \\
\text { by Weight }\end{array}$ & $\begin{array}{c}\text { Ash } \\
\text { Percent } \\
\text { by Weight }\end{array}$ & $\begin{array}{c}\text { Cents per } \\
\text { Million Btu }\end{array}$ & $\begin{array}{l}\text { Dollars per } \\
\text { Short Ton }\end{array}$ \\
\hline & \multicolumn{6}{|c|}{ Company and Plant: Public Serv Electric \& Gas Co, Mercer } \\
\hline \multicolumn{7}{|l|}{1998} \\
\hline 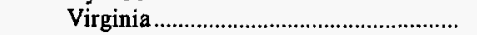 & 174.3 & 13,912 & 0.76 & 5.07 & 160.3 & 44.60 \\
\hline West Virginia & 8.1 & 11,531 & .67 & 10.60 & 126.9 & 29.27 \\
\hline Venezuela & 39.0 & 12,998 & .68 & 5.50 & 155.3 & 40.37 \\
\hline Total & 221.4 & 13,664 & .74 & $\mathbf{5 . 3 4}$ & 158.4 & 43.29 \\
\hline \multicolumn{7}{|l|}{ April - June } \\
\hline 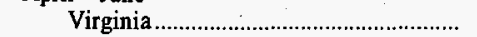 & 235.8 & 14,074 & .73 & 4.71 & 153.7 & 43.26 \\
\hline West Virginia & 32.7 & 11,555 & .70 & 15.96 & 127.6 & 29.49 \\
\hline Total & 268.5 & 13,767 & .73 & 6.08 & 151.0 & 41.58 \\
\hline \multicolumn{7}{|l|}{ Year to Date } \\
\hline 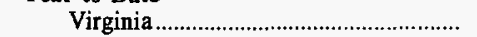 & 410.1 & 14,005 & .74 & 4.86 & 156.5 & 43.83 \\
\hline 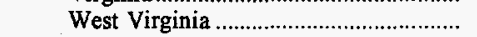 & 40.8 & 11,551 & .70 & 14.90 & 127.5 & 29.44 \\
\hline 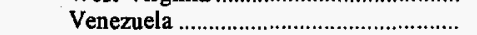 & 39.0 & 12,998 & .68 & 5.50 & 155.3 & 40.37 \\
\hline \multirow[t]{2}{*}{ 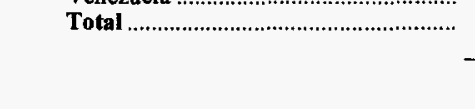 } & 489.9 & 13,721 & .73 & $\mathbf{5 . 7 5}$ & 154.4 & 42.36 \\
\hline & \multicolumn{6}{|c|}{ Company and Plant: Public Serv Electric \& Gas-NJ, Hudson } \\
\hline 1992 & & & & & & \\
\hline 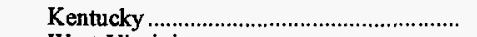 & 189.1 & 13,197 & 0.83 & 6.64 & 183.3 & 48.38 \\
\hline 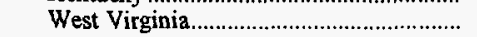 & 380.3 & 13,069 & .82 & 7.36 & 173.1 & 45.24 \\
\hline Total & 569.4 & 13,111 & .82 & 7.12 & 176.5 & 46.28 \\
\hline \multicolumn{7}{|l|}{1993} \\
\hline 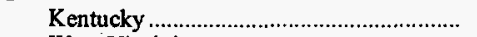 & 76.0 & 13,336 & .84 & 6.75 & 185.7 & 49.54 \\
\hline 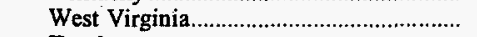 & 362.0 & 12,930 & .81 & 7.93 & 188.1 & 48.65 \\
\hline 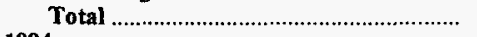 & 438.0 & 13,000 & .82 & 7.73 & 187.7 & 48.80 \\
\hline \multicolumn{7}{|l|}{1994} \\
\hline Kentucky & 251.3 & 13,158 & .73 & 7.48 & 202.1 & 53.19 \\
\hline West Virginia & 293.6 & 13,102 & .80 & 7.53 & 202.5 & 53.05 \\
\hline 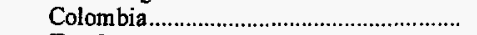 & 22.5 & 12,870 & .68 & 6.90 & 166.9 & 42.96 \\
\hline Total & 567.4 & 13,118 & .77 & 7.48 & 200.9 & $\mathbf{5 2 . 7 1}$ \\
\hline 1995 & & & & & & \\
\hline 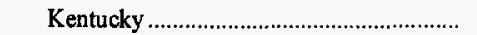 & 436.3 & 13,082 & .65 & 6.77 & 191.2 & 50.02 \\
\hline 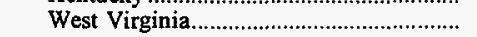 & 252.1 & 13,070 & .83 & 7.59 & 179.5 & 46.93 \\
\hline Total & 688.4 & 13,078 & .71 & 7.07 & 186.9 & 48.89 \\
\hline 1996 & & & & & & \\
\hline 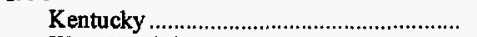 & 172.8 & 13,008 & .67 & 7.51 & 178.4 & 46.41 \\
\hline 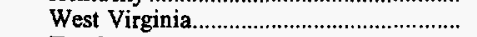 & 536.8 & 12,588 & .88 & 10.65 & 170.4 & 42.90 \\
\hline Total & 709.6 & 12,690 & .83 & 9.88 & 172.4 & 43.76 \\
\hline 1997 & & & & & & \\
\hline 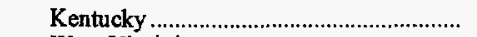 & 106.0 & 13,059 & .65 & 7.47 & 174.3 & 45.53 \\
\hline 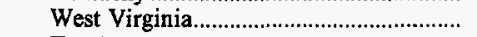 & 314.4 & 12,522 & .92 & 11.43 & 167.6 & 41.97 \\
\hline 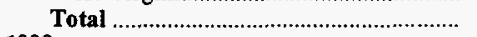 & 420.4 & 12,657 & .85 & 10.43 & 169.3 & 42.87 \\
\hline 1998 & & & & & & \\
\hline January - March & & & & & & \\
\hline Kentucky & 26.6 & 12,893 & .85 & 5.64 & 164.7 & 42.47 \\
\hline 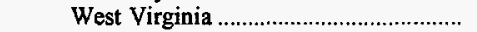 & 55.8 & 12,202 & .84 & 12.59 & 149.0 & 36.36 \\
\hline 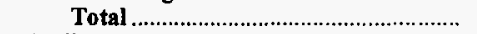 & 82.4 & 12,425 & .84 & 10.34 & 154.2 & 38.33 \\
\hline April - June & & & & & & \\
\hline 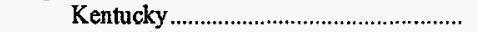 & 1.9 & 13,051 & .90 & 7.10 & 154.2 & 40.25 \\
\hline 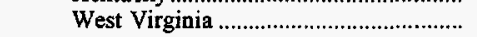 & 128.7 & 12,308 & .87 & 13.35 & 137.5 & 33.84 \\
\hline 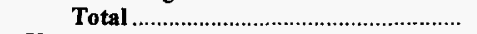 & 130.6 & 12,319 & .87 & 13.26 & 137.7 & 33.94 \\
\hline Year to Date & & & & & & \\
\hline Kentucky & 28.5 & 12,904 & .85 & 5.73 & 164.0 & 42.33 \\
\hline 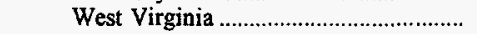 & 184.5 & 12,276 & .86 & 13.12 & 140.9 & 34.60 \\
\hline 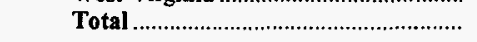 & 213.0 & 12,360 & .86 & 12.13 & 144.2 & 35.64 \\
\hline & & Company and & Savannah E & and Power, 1 & entworth & \\
\hline 1992 & & & & & & \\
\hline 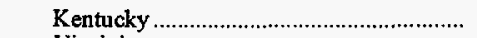 & 3.0 & 11,947 & 1.36 & 13.60 & 132.2 & 31.59 \\
\hline 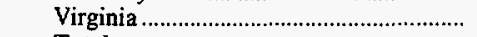 & 60.5 & 12,392 & .98 & 11.96 & 148.1 & 36.71 \\
\hline 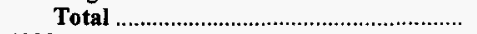 & 63.5 & 12,371 & 1.00 & 12.04 & 147.4 & 36.46 \\
\hline 1993 & & & & & & \\
\hline Kentucky & 80.2 & 12,770 & .98 & 9.66 & 175.8 & 44.91 \\
\hline Virginia & 174.6 & 12,782 & .99 & 10.15 & 173.0 & 44.22 \\
\hline 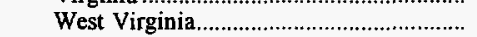 & 5.1 & 12,738 & .77 & 8.60 & 166.7 & 42.46 \\
\hline Total & 259.9 & 12,777 & .98 & 9.97 & 173.7 & 44.40 \\
\hline
\end{tabular}

See footnotes at the end of Table A7. 
Table A7. Cost and Quality of All Coal Received at Electric Utility Plants that Import Coal by Origin, 1992-1998 (Continued)

\begin{tabular}{|c|c|c|c|c|c|c|}
\hline \multirow[b]{2}{*}{$\begin{array}{l}\text { Time Period and State } \\
\text { or Country of Origin }\end{array}$} & \multirow{2}{*}{$\begin{array}{l}\text { Quantity } \\
\text { (thousand } \\
\text { short tons) }\end{array}$} & \multicolumn{3}{|c|}{ Average Quality 1} & \multicolumn{2}{|c|}{ Average Cost Delivered } \\
\hline & & $\begin{array}{c}\text { Btu } \\
\text { per Pound }\end{array}$ & $\begin{array}{c}\text { Sulfur } \\
\text { Percent } \\
\text { by Weight }\end{array}$ & $\begin{array}{c}\text { Ash } \\
\text { Percent } \\
\text { by Weight }\end{array}$ & $\begin{array}{l}\text { Cents per } \\
\text { Million Btu }\end{array}$ & $\begin{array}{l}\text { Dollars per } \\
\text { Short Ton }\end{array}$ \\
\hline & \multicolumn{6}{|c|}{ Company and Plant: Savannah Eleetric and Power, Port Wentworth } \\
\hline \multicolumn{7}{|l|}{1994} \\
\hline 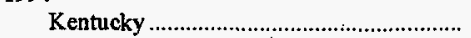 & 106.7 & 12,520 & 1.19 & 9.54 & 172.2 & 43.13 \\
\hline Virginia & 31.6 & 12,543 & .98 & 10.23 & 169.4 & 42.49 \\
\hline 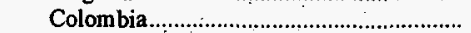 & 11.9 & 11,235 & .69 & 5.87 & 214.1 & 48.12 \\
\hline 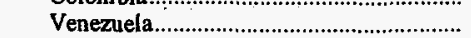 & 16.8 & 12,575 & 1.12 & 8.60 & 168.0 & 42.25 \\
\hline Total & 167.0 & 12,438 & 1.11 & 9.31 & 174.0 & 43.27 \\
\hline \multicolumn{7}{|l|}{1995} \\
\hline 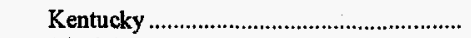 & 9.9 & 11,801 & .55 & 14.50 & 143.0 & 33.75 \\
\hline 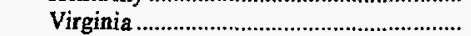 & 130.3 & 13,124 & .87 & 9.43 & 159.0 & 41.74 \\
\hline Total & 140.3 & 13,030 & .85 & 9.79 & 158.0 & 41.17 \\
\hline \multicolumn{7}{|l|}{1996} \\
\hline 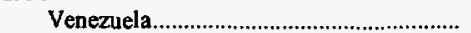 & 209.9 & 12,143 & 1.08 & 6.71 & 152.8 & 37.11 \\
\hline Total & 209.9 & 12,143 & 1.08 & 6.71 & 152.8 & 37.11 \\
\hline \multicolumn{7}{|l|}{1997} \\
\hline 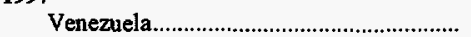 & 279.1 & 11,949 & 1.28 & 7.72 & 135.1 & 32.29 \\
\hline Total & 279.1 & 11,949 & 1.28 & 7.72 & 135.1 & 32.29 \\
\hline \multicolumn{7}{|l|}{1998} \\
\hline \multicolumn{7}{|l|}{ January - March } \\
\hline 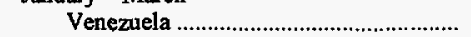 & 79.0 & 13,018 & .67 & 5.50 & 152.4 & 39.68 \\
\hline Total & 79.0 & 13,018 & .67 & 5.50 & 152.4 & 39.68 \\
\hline \multicolumn{7}{|l|}{ April - Jane } \\
\hline 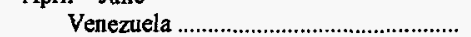 & 71.4 & 11,986 & 1.58 & 8.20 & 147.8 & 35.43 \\
\hline Total & 71.4 & 11,986 & 1.58 & 8.20 & 147.8 & 35.43 \\
\hline \multicolumn{7}{|l|}{ Year to Date } \\
\hline 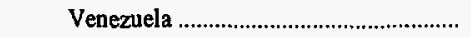 & 150.3 & 12,528 & 1.10 & 6.78 & 150.3 & 37.66 \\
\hline 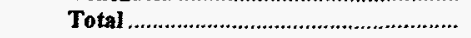 & 150.3 & 12,528 & 1.10 & 6.78 & 150.3 & 37.66 \\
\hline & & Company an & t: Takoma D & Public Utiliti & $\mathrm{m} \mathrm{No.2}$ & \\
\hline 1992 & & & & & & \\
\hline 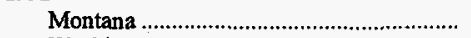 & 4.0 & 9,492 & 0.40 & 4.25 & 169.0 & 32,08 \\
\hline 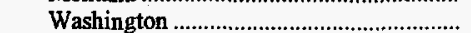 & 2.3 & 12,366 & .72 & 14.03 & 154.5 & 38.21 \\
\hline 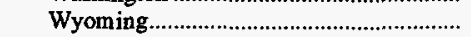 & 2.0 & 8,846 & .22 & 4.67 & 181.0 & 32.02 \\
\hline 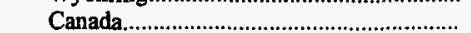 & 15.3 & 9,993 & .42 & 12.95 & 214.7 & 42.90 \\
\hline 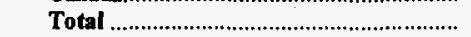 & 23.7 & 10,043 & .43 & 10.87 & 197.5 & 39.67 \\
\hline 1993 & & & & & & \\
\hline Montana & 10.0 & 9,482 & .37 & 4.10 & 182.6 & 34.63 \\
\hline Washington & 2.2 & 10,967 & .70 & 14.47 & 163.5 & 35.87 \\
\hline 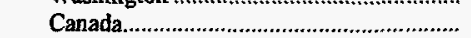 & 29.2 & 10,036 & .48 & 12.60 & 179.5 & 36.03 \\
\hline Total & 41.4 & 9,951 & .46 & 10.64 & 179.3 & 35.68 \\
\hline 1994 & & & & & & \\
\hline 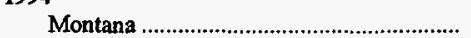 & 26.4 & 9,465 & .41 & 4.63 & 175.8 & 33.27 \\
\hline Washington & 3.3 & 10,865 & .72 & 13.30 & 165.3 & 35.91 \\
\hline 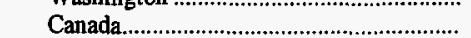 & 6.3 & 9,806 & .48 & 12.80 & 178.0 & 34.91 \\
\hline 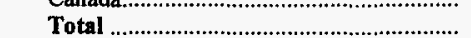 & 36.1 & 9,655 & .45 & $\begin{array}{r}12.00 \\
6.87\end{array}$ & 175.1 & 33.81 \\
\hline 1995 & & & & & & \\
\hline Montana & 3.8 & 9,470 & .36 & 4.64 & 180.0 & 34.09 \\
\hline Canada & 23.8 & 10,066 & .47 & $\begin{array}{r}4.04 \\
13.14\end{array}$ & 166.0 & 34,42 \\
\hline Total & 27.6 & 9,983 & .46 & 11.96 & 167.8 & 33.51 \\
\hline 1996 & & & & & & \\
\hline 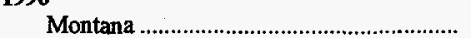 & 3.9 & 9,516 & .50 & 5.00 & 176.0 & 33.50 \\
\hline 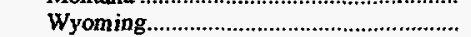 & .3 & 8,858 & .27 & 5.13 & 109.0 & 19.31 \\
\hline 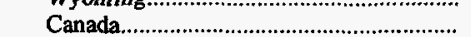 & 18.0 & 9,861 & .44 & 12.97 & 174.6 & 34.44 \\
\hline 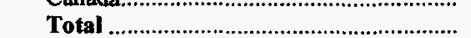 & 22.2 & 9,788 & .45 & 11.47 & 174.1 & 34,09 \\
\hline 1997 & & & & & & \\
\hline Washington & 1.5 & 10,616 & .70 & 17.57 & 154.0 & 32.71 \\
\hline 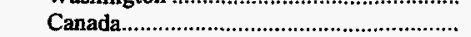 & 9.6 & 10,144 & .43 & 12.25 & 171.4 & 34.79 \\
\hline Total & 11.1 & 10,208 & .46 & 12.96 & 169.0 & 34.51 \\
\hline 1998 & & & & & & \\
\hline January - March & & & & & & \\
\hline 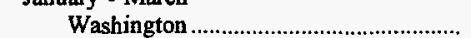 & 8 & 10,800 & .73 & 72.92 & 165.2 & 35.67 \\
\hline Total & .8 & 10,800 & .73 & 72.92 & 165.2 & 35.67 \\
\hline April - June & & & & & & \\
\hline 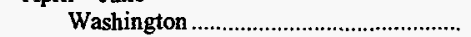 & .1 & 10,800 & .73 & 14.00 & 168.0 & 36.29 \\
\hline Total & .1 & 10,800 & .73 & 14.00 & 168.0 & 36.29 \\
\hline
\end{tabular}

See footnotes at the end of Table A7. 
Table A7. Cost and Quality of All Coal Received at Electric Utility Plants that Import Coal by Origin, 1992-1998 (Continued)

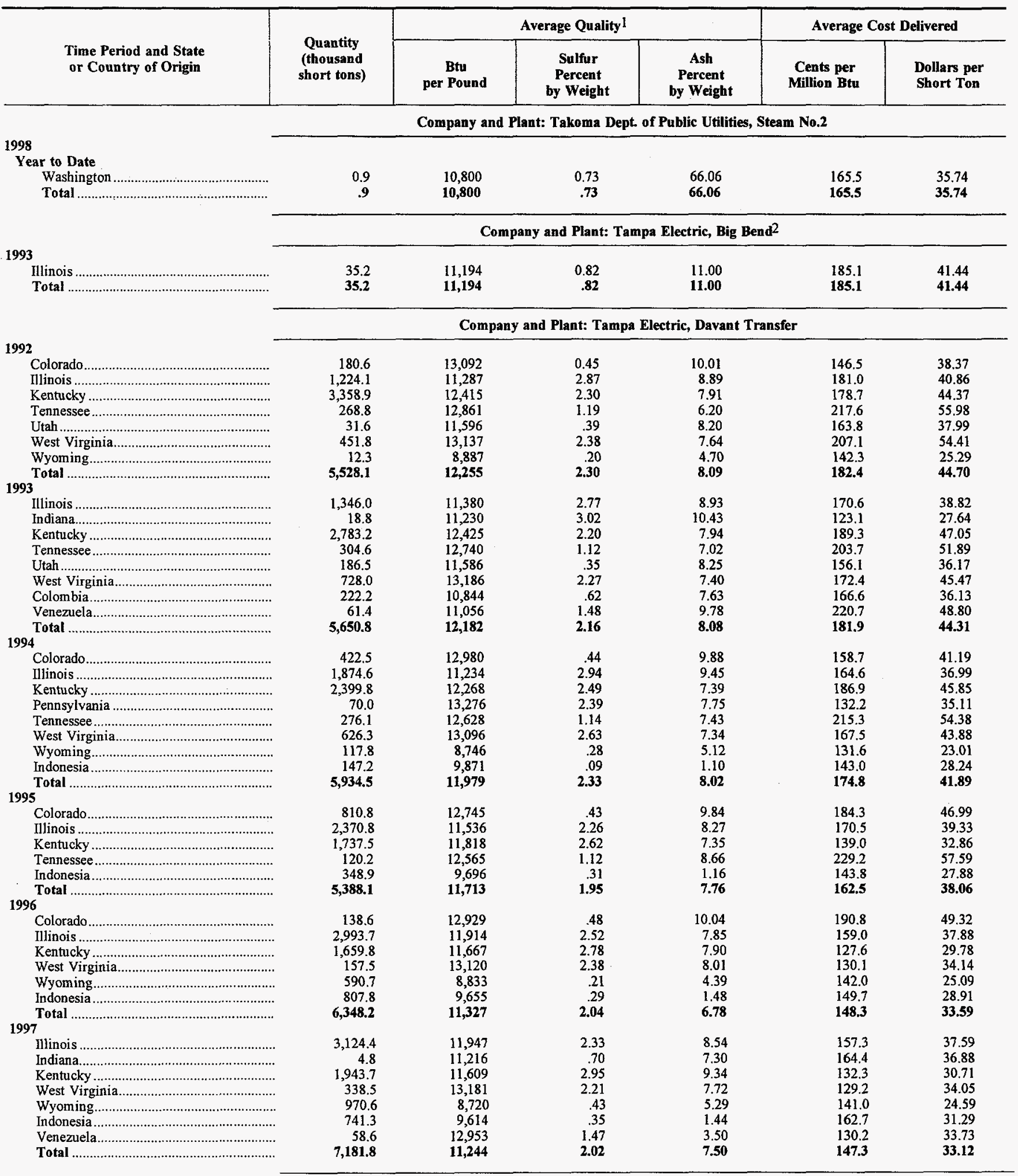

See footnotes at the end of Table A7. 
Table A7. Cost and Quality of All Coal Received at Electric Utility Plants that Import Coal by Origin, 1992-1998 (Continued)

\begin{tabular}{|c|c|c|c|c|c|c|}
\hline \multirow[b]{2}{*}{$\begin{array}{l}\text { Time Period and State } \\
\text { or Country of Origin }\end{array}$} & \multirow[b]{2}{*}{$\begin{array}{l}\text { Quantity } \\
\text { (thousand } \\
\text { short tons) }\end{array}$} & \multicolumn{3}{|c|}{ Average Quality 1} & \multicolumn{2}{|c|}{ Average Cost Delivered } \\
\hline & & $\begin{array}{c}\text { Btu } \\
\text { per Pound }\end{array}$ & $\begin{array}{c}\text { Sulfur } \\
\text { Percent } \\
\text { by Weight }\end{array}$ & $\begin{array}{c}\text { Ash } \\
\text { Percent } \\
\text { by Weight }\end{array}$ & $\begin{array}{l}\text { Cents per } \\
\text { Million Btu }\end{array}$ & $\begin{array}{l}\text { Dollars per } \\
\text { Short Ton }\end{array}$ \\
\hline & \multicolumn{6}{|c|}{ Company and Plant: Tampa Electric, Davant Transfer } \\
\hline \multicolumn{7}{|l|}{$\begin{array}{l}1998 \\
\quad \text { January - March }\end{array}$} \\
\hline 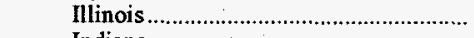 & 861.7 & 11,870 & 2.43 & 8.47 & 160.9 & 38.21 \\
\hline Indiana & 30.4 & 10,864 & 3.03 & 10.20 & 125.2 & 27.20 \\
\hline 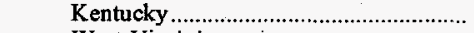 & 561.5 & 11,491 & 2.68 & 7.83 & 130.4 & 29.98 \\
\hline 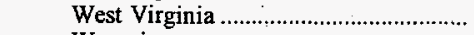 & 158.3 & 13,214 & 2.25 & 7.56 & 137.5 & 36.33 \\
\hline Wyoming & 252.3 & 8,749 & .40 & 5.33 & 138.2 & 24.19 \\
\hline 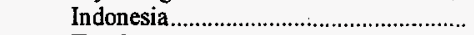 & 73.8 & 9,507 & .38 & 1.10 & 163.3 & 31.05 \\
\hline Total & $1,938.0$ & 11,358 & 2.15 & 7.55 & 147.0 & 33.40 \\
\hline \multicolumn{7}{|l|}{ April - June } \\
\hline 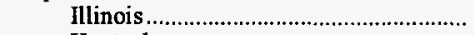 & 866.7 & 11,991 & 2.38 & 8.29 & 156,1 & 37.43 \\
\hline Kentucky & 539.0 & 11,554 & 2.76 & 7.93 & 128.4 & 29.68 \\
\hline 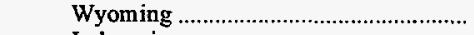 & 225.7 & 8,721 & .40 & 5.11 & 133.8 & 23.34 \\
\hline 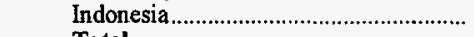 & 223.5 & 9,623 & .21 & 1.13 & 157.9 & 30.38 \\
\hline Total & $1,854.8$ & 11,181 & 1.99 & 6.94 & 145.9 & 32.61 \\
\hline \multicolumn{7}{|l|}{ Year to Date } \\
\hline 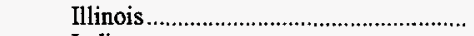 & $1,728.4$ & 11,931 & 2.41 & 8.38 & 158.5 & 37.82 \\
\hline Indiana & 30.4 & 10,864 & 3.03 & 10.20 & 125.2 & 27.20 \\
\hline 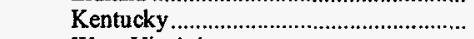 & $1,100.5$ & 11,522 & 2.72 & 7.88 & 129.5 & 29.83 \\
\hline 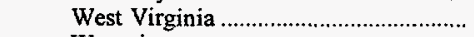 & 158.3 & 13,214 & 2.25 & 7.56 & 137.5 & 36.33 \\
\hline 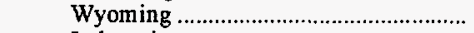 & 477.9 & 8,735 & .40 & 5.23 & 136.2 & 23.79 \\
\hline Indonesia & 297.3 & 9,594 & .25 & 1.12 & 159.2 & 30.55 \\
\hline \multirow[t]{2}{*}{ 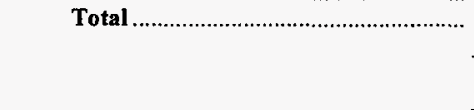 } & $3,792.8$ & 11,271 & 2.07 & 7.25 & 146.5 & 33.02 \\
\hline & \multicolumn{6}{|c|}{ Company and Plant: United Illuminating Co, Bridgeport Harbor } \\
\hline \multicolumn{7}{|l|}{1992} \\
\hline 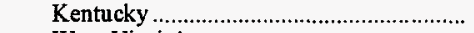 & 772.0 & 13,162 & 0.55 & 6.25 & 195.5 & 51.47 \\
\hline 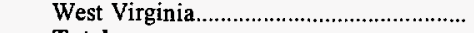 & 21.0 & 13,351 & .67 & 6.20 & 168.9 & 45.10 \\
\hline Total & 793.0 & 13,167 & .55 & 6.25 & 194.8 & $\mathbf{5 1 . 3 0}$ \\
\hline \multicolumn{7}{|l|}{1993} \\
\hline 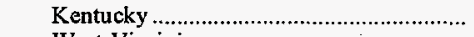 & 665.0 & 13,113 & .54 & 7.02 & 170.6 & 44.74 \\
\hline West Virginia....................................... & 75.0 & 13,426 & .67 & 6.33 & 168.6 & 45.29 \\
\hline Total & 740.0 & 13,144 & .55 & 6.95 & 170.4 & 44.80 \\
\hline 1994 & & & & & & \\
\hline 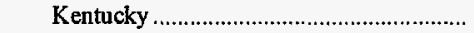 & 809.0 & 13,080 & .53 & 7.41 & 177.6 & 46.46 \\
\hline 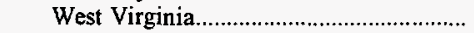 & 54.0 & 13,306 & .64 & 6.97 & 173.8 & 46.25 \\
\hline Total & 863.0 & 13,094 & .54 & 7.38 & 177.4 & 46.45 \\
\hline 1995 & & & & & & \\
\hline 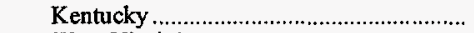 & 836.0 & 13,109 & .56 & 7.05 & 188.1 & 49.32 \\
\hline 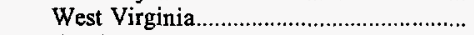 & 5.0 & 13,252 & .69 & 6.50 & 190.0 & 50.36 \\
\hline 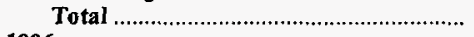 & 841.0 & 13,110 & .56 & 7.05 & 188.1 & 49.33 \\
\hline 1996 & & & & & & \\
\hline 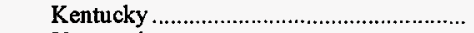 & 903.0 & 13,098 & .54 & 7.24 & 191.2 & 50.09 \\
\hline Venezuela & 28.0 & 13,174 & .61 & 4.10 & 185.0 & 48.74 \\
\hline Total & 931.0 & 13,100 & .54 & 7.14 & 191.0 & 50.05 \\
\hline 1997 & & & & & & \\
\hline 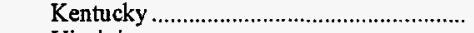 & 760.0 & 13,094 & .51 & 7.41 & 191.9 & 50.25 \\
\hline Virginia & 4.0 & 13,830 & .72 & 5.27 & 184.2 & 50.94 \\
\hline West Virginia & 153.1 & 13,244 & .63 & 6.85 & 188.5 & 49.93 \\
\hline 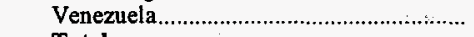 & 35.0 & 13,387 & .64 & 4.30 & 169.6 & 45.41 \\
\hline Total & 952.1 & 13,132 & .54 & $\mathbf{7 . 2 0}$ & 190.5 & 50.02 \\
\hline 1998 & & & & & & \\
\hline January - March & & & & & & \\
\hline Kentucky & 229.0 & 13,113 & .51 & 6.89 & 184.3 & 48.33 \\
\hline 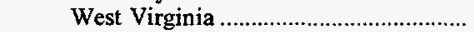 & 49.0 & 13,246 & .61 & 7.67 & 181.3 & 48.04 \\
\hline Total & 278.0 & 13,137 & .53 & 7.03 & 183.7 & 48.28 \\
\hline April - June & & & & & & \\
\hline Kentucky & 123.0 & 13,076 & .49 & 7.16 & 180.6 & 47.24 \\
\hline 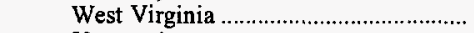 & 15.0 & 13,346 & .63 & 7.03 & 181.6 & 48.47 \\
\hline 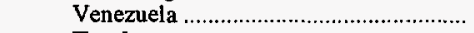 & 36.0 & 13,677 & .58 & 4.00 & 161.6 & 44.20 \\
\hline Total & 174.0 & 13,223 & .52 & 6.50 & 176.6 & 46.72 \\
\hline Year to Date & & & & & & \\
\hline 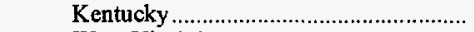 & 352.0 & 13,100 & .50 & 6.99 & 183.0 & 47.95 \\
\hline West Virginia & 64.0 & 13,270 & .61 & 7.52 & 181.4 & 48.14 \\
\hline 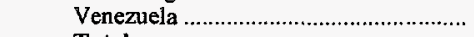 & 36.0 & 13,677 & .58 & 4.00 & 161.6 & 44.20 \\
\hline Total & 452.0 & 13,170 & .52 & 6.82 & 181.0 & 47.68 \\
\hline
\end{tabular}

See footnotes at the end of Table A7. 
Table A7. Cost and Quality of All Coal Received at Electric Utility Plants that Import Coal by Origin, 1992-1998 (Continued)

\begin{tabular}{|c|c|c|c|c|c|c|}
\hline \multirow[b]{2}{*}{$\begin{array}{l}\text { Time Period and State } \\
\text { or Country of Origin }\end{array}$} & \multirow{2}{*}{$\begin{array}{l}\text { Quantity } \\
\text { (thousand } \\
\text { short tons) }\end{array}$} & \multicolumn{3}{|c|}{ Average Quality 1} & \multicolumn{2}{|c|}{ Average Cost Delivered } \\
\hline & & $\begin{array}{c}\text { Btu } \\
\text { per Pound }\end{array}$ & $\begin{array}{c}\text { Sulfur } \\
\text { Percent } \\
\text { by Weight }\end{array}$ & $\begin{array}{c}\text { Ash } \\
\text { Percent } \\
\text { by Weight }\end{array}$ & $\begin{array}{l}\text { Cents per } \\
\text { Million Btu }\end{array}$ & $\begin{array}{l}\text { Dollars per } \\
\text { Short Ton }\end{array}$ \\
\hline & \multicolumn{6}{|c|}{ Total of U.S. Electric Utility Plants } \\
\hline \multicolumn{7}{|l|}{1995} \\
\hline 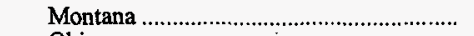 & $1,273.3$ & 9,400 & 0.38 & 4.43 & 140.1 & 26.34 \\
\hline 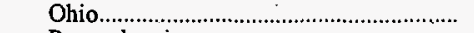 & 225.7 & 12,444 & 3.78 & 10.17 & 95,2 & 23.68 \\
\hline 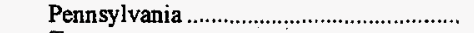 & $2,051.9$ & 13,140 & 1.78 & 7.51 & 137.1 & 36.04 \\
\hline Tennessee & 120.2 & 12,565 & 1.12 & 8.66 & 229.2 & 57.59 \\
\hline 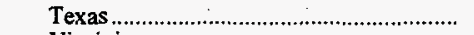 & 40.0 & 10,540 & 1.03 & 21.69 & 120.2 & 25.33 \\
\hline 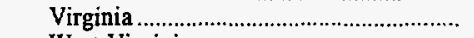 & 172.6 & 13,245 & .93 & 8.77 & 162.0 & 42.92 \\
\hline 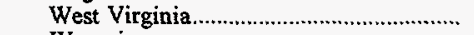 & $8,366.4$ & 12,623 & .81 & 10.13 & 162.4 & 40.99 \\
\hline 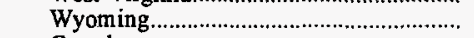 & $11,861.9$ & 8,453 & .35 & 5.32 & 134.3 & 22.71 \\
\hline Canada & 23.8 & 10,066 & .47 & 13.14 & 166.0 & 33.42 \\
\hline Colombia & $2,040.1$ & 11,985 & .65 & 6.83 & 153.9 & 36.89 \\
\hline Indonesia & 428.6 & 10,181 & .35 & 1.79 & 149.2 & 30.37 \\
\hline 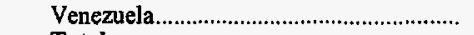 & $1,905.7$ & 12,610 & .79 & 6.57 & 194.1 & 48.95 \\
\hline 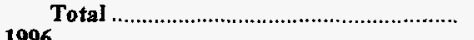 & $45,625.9$ & 11,239 & .90 & 7.46 & 157.8 & 35.48 \\
\hline \multicolumn{7}{|l|}{1996} \\
\hline Colorado & $2,481.0$ & 10,807 & .41 & 6.64 & 143.1 & 30.94 \\
\hline Illinois & $5,346.5$ & 11,951 & 2.04 & 7.42 & 171.3 & 40.95 \\
\hline Indiana & 18.1 & 11,256 & 1.58 & 7.15 & 97.7 & 21.99 \\
\hline Kentucky & $10,037.7$ & 12,543 & 1.34 & 8.53 & 162.6 & 40.80 \\
\hline 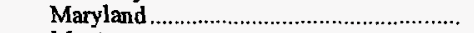 & 277.2 & 13,143 & 1.37 & 9.31 & 150.2 & 39.49 \\
\hline Montana & $2,167.2$ & 9,394 & .40 & 4.48 & 141.1 & 26.51 \\
\hline Ohio & 2.3 & 11,043 & 2.57 & 10.80 & 72.6 & 16.03 \\
\hline 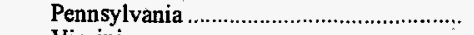 & $1,940.2$ & 13,163 & 1.75 & 7.34 & 142.7 & 37.56 \\
\hline Virginia & 17.9 & 14,102 & .74 & 5.76 & 201.2 & 56.74 \\
\hline 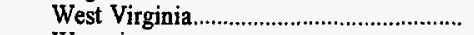 & $10,499.4$ & 12,578 & 1.07 & 10.23 & 153.6 & 38.63 \\
\hline 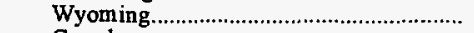 & $12,299.2$ & 8,456 & .37 & 5.55 & 130.7 & 22.11 \\
\hline 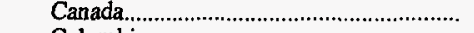 & 18.0 & 9,861 & .44 & 12.97 & 174.6 & 34.44 \\
\hline 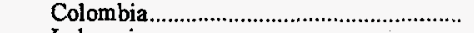 & $2,079.9$ & 11,883 & .64 & 7.04 & 155.7 & 37.01 \\
\hline 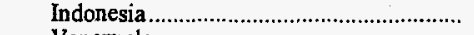 & 833.7 & 9,741 & .31 & 1.68 & 150.2 & 29.26 \\
\hline 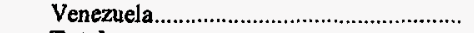 & $1,767.6$ & 12,686 & .77 & 6.14 & 171.7 & 43.58 \\
\hline Total & $49,786.0$ & 11,211 & .98 & 7.46 & 152.7 & 34.24 \\
\hline \multicolumn{7}{|l|}{1997} \\
\hline 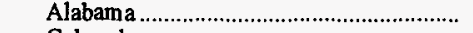 & 362.4 & 11,887 & 2.03 & 13.36 & 177.4 & 42.18 \\
\hline Colorado & $1,531.4$ & 10,289 & .38 & 6.11 & 135.8 & 27.95 \\
\hline Illinois & $4,586.4$ & 11,966 & 1.99 & 8.03 & 172.4 & 41.26 \\
\hline 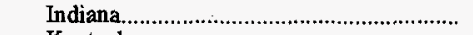 & 10.0 & 11,077 & 1.51 & 8.06 & 135.2 & 29.96 \\
\hline 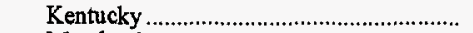 & $8,984.0$ & 12,435 & 1.52 & 9.00 & 162.8 & 40.48 \\
\hline Maryland & 160.8 & 13,158 & 1.46 & 9.17 & 147.8 & 38.89 \\
\hline Montana & $3,221.3$ & 9,323 & .39 & 4.56 & 145.5 & 27.13 \\
\hline Ohio & 201.5 & 11,728 & 3.06 & 12.49 & 92.6 & 21.73 \\
\hline 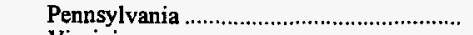 & $2,507.1$ & 12,966 & 1.70 & 8.40 & 139.6 & 36.21 \\
\hline Virginia & 201.4 & 13,250 & .88 & 8.73 & 160.1 & 42.43 \\
\hline Washington & 1.5 & 10,616 & .70 & 17.57 & 154.0 & 32.71 \\
\hline 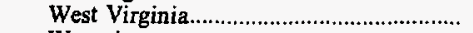 & $10,051.5$ & 12,602 & .91 & 10.55 & 156.8 & 39.53 \\
\hline Wyoming & $12,440.9$ & 8,464 & .41 & 5.54 & 128.6 & 21.76 \\
\hline 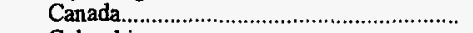 & 9.6 & 10,144 & .43 & 12.25 & 171.4 & 34.79 \\
\hline 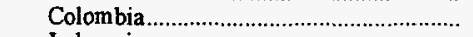 & $2,744.4$ & 12,026 & .70 & 6.71 & 158.8 & 38.18 \\
\hline 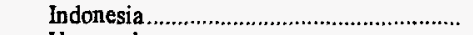 & 782.0 & 9,754 & .36 & 1.60 & 164.6 & 32.10 \\
\hline 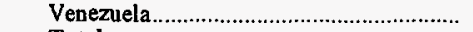 & $1,335.1$ & 12,722 & .83 & 6.40 & 158.7 & 40.38 \\
\hline Total & $49,131.3$ & 11,116 & .98 & 7.67 & 152.1 & 33.81 \\
\hline \multirow{2}{*}{\multicolumn{7}{|c|}{1998}} \\
\hline & & & & & & \\
\hline Alabama & 36.4 & 11,686 & 1.87 & 14.34 & 182.6 & 42.69 \\
\hline 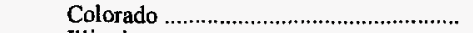 & 376.4 & 10,469 & .35 & 5.80 & 140.8 & 29.49 \\
\hline Illinois. & $1,296.6$ & 11,976 & 2.06 & 7.89 & 169.4 & 40.59 \\
\hline Indiana & 30.4 & 10,864 & 3.03 & 10.20 & 125.2 & 27.20 \\
\hline 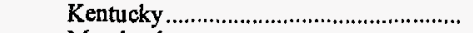 & $2,525.3$ & 12,401 & 1.42 & 8.57 & 159.5 & 39.55 \\
\hline 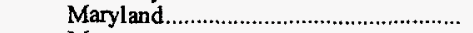 & 7.1 & 13,367 & 1.56 & 9.00 & 146.1 & 39.06 \\
\hline Montana & 801.7 & 9,421 & .39 & 4.65 & 149.0 & 28.07 \\
\hline Ohio & 63.0 & 11,992 & 3.02 & 11.70 & 98.5 & 23.62 \\
\hline 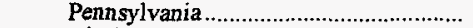 & 652.7 & 13,108 & 1.71 & 7.51 & 137.6 & 36.06 \\
\hline 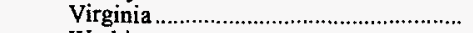 & 27.8 & 13,259 & .81 & 7.87 & 171.9 & 45.58 \\
\hline 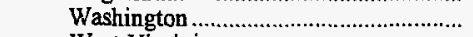 & .8 & 10,800 & .73 & 72.92 & 165.2 & 35.67 \\
\hline West Virginia & $2,444.2$ & 12,627 & .97 & 10.15 & 152.2 & 38.44 \\
\hline 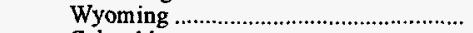 & $3,281.6$ & 8,471 & .40 & 5.55 & 125.3 & 21.22 \\
\hline 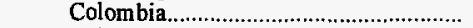 & 680.8 & 12,049 & .63 & 6.61 & 160.8 & 38.74 \\
\hline Indonesia & 73.8 & 9,507 & .38 & 1.10 & 163.3 & 31.05 \\
\hline 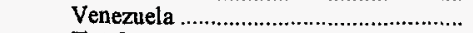 & 611.0 & 13,112 & .66 & 6.29 & 158.6 & 41.59 \\
\hline Total & $12,909.8$ & 11,190 & .99 & 7.44 & 149.8 & 33.52 \\
\hline
\end{tabular}

See footnotes at the end of Table A7 
Table A7. Cost and Quality of All Coal Received at Electric Utility Plants that Import Coal by Origin, 1992-1998 (Continued)

\begin{tabular}{|c|c|c|c|c|c|c|}
\hline \multirow[b]{2}{*}{$\begin{array}{l}\text { Time Period and State } \\
\text { or Country of Origin }\end{array}$} & \multirow{2}{*}{$\begin{array}{c}\text { Quantity } \\
\text { (thousand } \\
\text { short tons) }\end{array}$} & \multicolumn{3}{|c|}{ Average Quality 1} & \multicolumn{2}{|c|}{ Average Cost Delivered } \\
\hline & & $\begin{array}{c}\text { Btu } \\
\text { per Pound }\end{array}$ & $\begin{array}{c}\text { Sulfur } \\
\text { Percent } \\
\text { by Weight }\end{array}$ & $\begin{array}{c}\text { Ash } \\
\text { Percent } \\
\text { by Weight }\end{array}$ & $\begin{array}{l}\text { Cents per } \\
\text { Million Btu }\end{array}$ & $\begin{array}{l}\text { Dollars per } \\
\text { Short Ton }\end{array}$ \\
\hline & \multicolumn{6}{|c|}{ Total of U.S. Electric Utility Plants } \\
\hline \multicolumn{7}{|l|}{1992} \\
\hline Alabama & 31.9 & 12,060 & 2.75 & 12.94 & 120.6 & 29.09 \\
\hline Colorado & $1,961.3$ & 11,088 & .40 & 6.66 & 198.7 & 44.06 \\
\hline 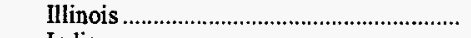 & $3,933.7$ & 11,729 & 2.79 & 8.55 & 190.4 & 44.67 \\
\hline 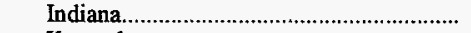 & 826.6 & 10,901 & 2.26 & 8.78 & 142.3 & 31.01 \\
\hline 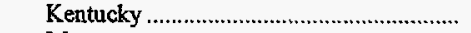 & $9,906.3$ & 12,654 & 1.44 & 7.99 & 170.5 & 43.14 \\
\hline 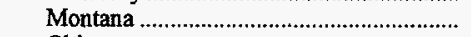 & 86.2 & 9,388 & .31 & 4.16 & 137.5 & 25.82 \\
\hline Ohio & 963.7 & 12,135 & 3.62 & 11.27 & 104.4 & 25.35 \\
\hline 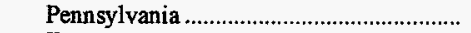 & $1,340.4$ & 13,123 & 1.60 & 7.13 & 167.3 & 43.92 \\
\hline 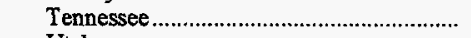 & 268.8 & 12,861 & 1.19 & 6.20 & 217.6 & 55.98 \\
\hline Utah & 31.6 & 11,596 & .39 & 8.20 & 163.8 & 37.99 \\
\hline Virginia & 761.6 & 12,926 & 1.03 & 8.27 & 176.4 & 45.60 \\
\hline Washington & 2.3 & 12,366 & .72 & 14.03 & 154.5 & 38.21 \\
\hline 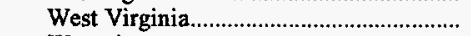 & $10,753.8$ & 12,869 & 1.00 & 9.20 & 168.0 & 43.25 \\
\hline 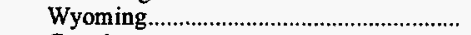 & $9,242.1$ & 8,427 & .40 & 5.34 & 138.8 & 23.40 \\
\hline 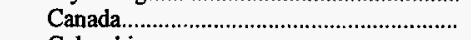 & 48.1 & 12,432 & 1.09 & 6.72 & 185.1 & 46.01 \\
\hline 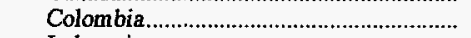 & $1,504.1$ & 11,938 & .70 & 6.91 & 150.9 & 36.04 \\
\hline Indonesia & 13.1 & 9,587 & .14 & 1.20 & 166.9 & 32.00 \\
\hline 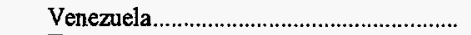 & 240.6 & 13,206 & .69 & 7.18 & 164.6 & 43.49 \\
\hline Total & $41,956.3$ & 11,561 & 1.21 & 7.72 & 165.2 & 38.19 \\
\hline \multicolumn{7}{|l|}{1993} \\
\hline Alabama & 72.3 & 12,337 & 2.09 & 11.73 & 191.1 & 47.15 \\
\hline 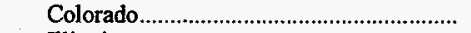 & $1,947.6$ & 10,661 & .40 & 6.86 & 198.9 & 42.40 \\
\hline Illinois & $3,596.4$ & 11,738 & 2.56 & 8.43 & 174.6 & 40.99 \\
\hline Indiana & 485.4 & 11,003 & 2.15 & 8.88 & 136.5 & 30.05 \\
\hline 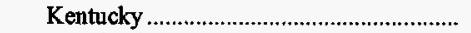 & $9,134.0$ & 12,697 & 1.33 & 8.12 & 174.0 & 44.19 \\
\hline Maryland & 56.2 & 13,015 & 1.30 & 9.55 & 161.4 & 42.00 \\
\hline Montana & 187.7 & 9,428 & .39 & 4.58 & 160.4 & 30.24 \\
\hline Ohio & $1,151.5$ & 12,135 & 3.57 & 11.37 & 102.2 & 24.81 \\
\hline Pennsylvania & $1,451.4$ & 13,093 & 1.79 & 7.35 & 156.7 & 41.04 \\
\hline 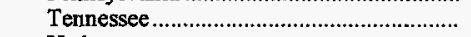 & 304.6 & 12,740 & 1.12 & 7.02 & 203.7 & 51.89 \\
\hline Utah & 186.5 & 11,586 & .35 & 8.25 & 156.1 & 36.17 \\
\hline 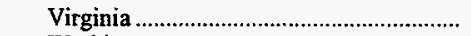 & 435.8 & 12,995 & .94 & 8.99 & 186.8 & 48.56 \\
\hline 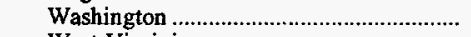 & 2.2 & 10,967 & .70 & 14.47 & 163.5 & 35.87 \\
\hline 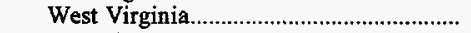 & $8,787.9$ & 12,904 & 1.03 & 8.97 & 167.0 & 43.10 \\
\hline Wyoming & $11,393.1$ & 8,389 & .38 & 5.24 & 134.5 & 22.56 \\
\hline 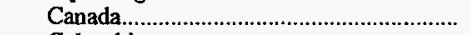 & 29.2 & 10,036 & .48 & 12.60 & 179.5 & 36.03 \\
\hline Colombia & $3,585.1$ & 11,867 & .66 & 6.85 & 149.0 & 35.37 \\
\hline 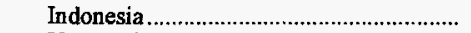 & 115.8 & 10,620 & .22 & 2.07 & 166.1 & 35.29 \\
\hline Venezuela & 897.5 & 12,874 & .67 & 6.96 & 166.4 & 42.84 \\
\hline Total & $43,820.3$ & 11,342 & 1.08 & $\mathbf{7 . 4 3}$ & 160.7 & 36.46 \\
\hline \multicolumn{7}{|l|}{1994} \\
\hline 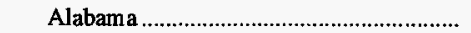 & 1.5 & 12,241 & 2.87 & 10.00 & 204.1 & 49.97 \\
\hline 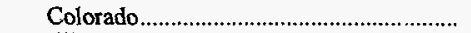 & $2,861.0$ & 11,189 & .42 & 8.16 & 181.7 & 40.67 \\
\hline 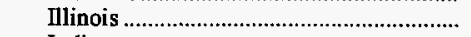 & $4,198.2$ & 11,616 & 2.44 & 8.40 & 164.4 & 38.19 \\
\hline Indiana & 326.8 & 11,062 & 1.82 & 8.77 & 121.5 & 26.88 \\
\hline 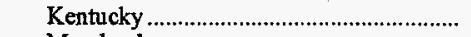 & $8,908.6$ & 12,635 & 1.37 & 8.25 & 176.8 & 44.67 \\
\hline 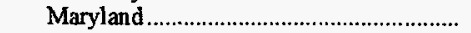 & 138.3 & 13,155 & 1.38 & 9.85 & 149.9 & 39.43 \\
\hline 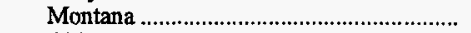 & $1,314.8$ & 9,404 & .40 & 4.78 & 138.8 & 26.11 \\
\hline Ohio & 937.8 & 12,266 & 3.58 & 10.63 & 99.0 & 24.28 \\
\hline Pennsylvania & $1,992.6$ & 13,125 & 1.76 & 7.34 & 144.5 & 37.94 \\
\hline 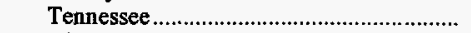 & 276.1 & 12,628 & 1.14 & 7.43 & 215.3 & 54.38 \\
\hline Virginia & 127.7 & 12,926 & .87 & 8.50 & 173.6 & 44.89 \\
\hline Washington & 3.3 & 10,865 & .72 & 13.30 & 165.3 & 35.91 \\
\hline 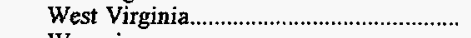 & $10,671.9$ & 12,732 & .95 & 9.58 & 165.3 & 42.08 \\
\hline 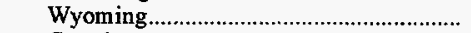 & $10,628.8$ & 8,440 & .35 & 5.15 & 133.6 & 22.55 \\
\hline Canada & 63.3 & 10,885 & .26 & 10.53 & 152.4 & 33.19 \\
\hline 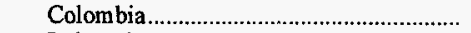 & $2,971.8$ & 11,997 & .66 & 6.76 & 142.7 & 34.25 \\
\hline 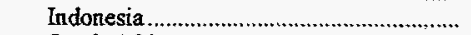 & 437.3 & 10,499 & .22 & 1.82 & 157.4 & 33.06 \\
\hline 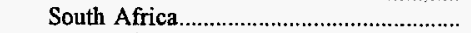 & 127.3 & 11,318 & .65 & 12.60 & 181.1 & 41.00 \\
\hline 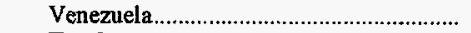 & $1,355.2$ & 12,649 & .76 & 6.61 & 172.3 & 43.60 \\
\hline Total & $47,342.4$ & 11,387 & 1.04 & 7.59 & 159.0 & 36.20 \\
\hline \multicolumn{7}{|l|}{1995} \\
\hline Colorado & $3,552.8$ & 11,471 & .42 & 8.39 & 170.5 & 39.12 \\
\hline Illinois & $4,594.4$ & 11,754 & 1.95 & 7.81 & 170.7 & 40.12 \\
\hline Indiana & 133.3 & 11,064 & 1.31 & 9.65 & 116.2 & 25.72 \\
\hline 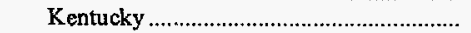 & $8,569.5$ & 12,623 & 1.27 & 8.01 & 165.8 & 41.86 \\
\hline 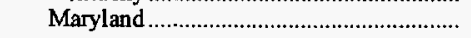 & 265.8 & 13,113 & 1.29 & 9.87 & 151.1 & 39.62 \\
\hline
\end{tabular}

See footnotes at the end of Table A7. 
Table A7. Cost and Quality of All Coal Received at Electric Utility Plants that Import Coal by Origin, 1992-1998 (Continued)

\begin{tabular}{|c|c|c|c|c|c|c|}
\hline \multirow[b]{2}{*}{$\begin{array}{l}\text { Time Period and State } \\
\text { or Country of Origin }\end{array}$} & \multirow{2}{*}{$\begin{array}{l}\text { Quantity } \\
\text { (thousand } \\
\text { short tons) }\end{array}$} & \multicolumn{3}{|c|}{ Average Qualityl } & \multicolumn{2}{|c|}{ Average Cost Delivered } \\
\hline & & $\begin{array}{c}\text { Btu } \\
\text { per Pound }\end{array}$ & $\begin{array}{c}\text { Sulfur } \\
\text { Percent } \\
\text { by Weight }\end{array}$ & $\begin{array}{c}\text { Ash } \\
\text { Percent } \\
\text { by Weight }\end{array}$ & $\begin{array}{c}\text { Cents per } \\
\text { Million Btu }\end{array}$ & $\begin{array}{l}\text { Dollars per } \\
\text { Short Ton }\end{array}$ \\
\hline & \multicolumn{6}{|c|}{ Total of U.S. Electric Utility Plants } \\
\hline \multicolumn{7}{|l|}{1998} \\
\hline \multicolumn{7}{|l|}{ April - June } \\
\hline 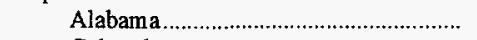 & 72.6 & 13,066 & 0.73 & 8.30 & 152.5 & 39.84 \\
\hline 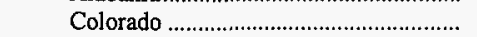 & 248.2 & 10,472 & .36 & 5.50 & 139.8 & 29.28 \\
\hline 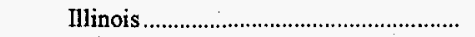 & $1,499.6$ & 12,048 & 1.94 & 7.72 & 153.7 & 37.04 \\
\hline 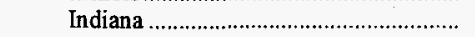 & 1.8 & 11,343 & 2.20 & 8.20 & 97.7 & 22.16 \\
\hline 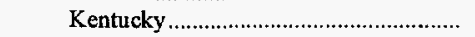 & $2,520.3$ & 12,482 & 1.43 & 8.45 & 156.6 & 39.10 \\
\hline Maryland & 49.5 & 13,028 & 1.46 & 8.56 & 146.3 & 38.13 \\
\hline 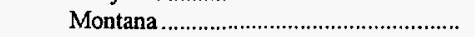 & 804.3 & 9,395 & .37 & 4.37 & 145.8 & 27.39 \\
\hline Ohio & 82.0 & 12,366 & 3.51 & 10.23 & 88.4 & 21.86 \\
\hline 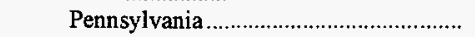 & 443.7 & 13,110 & 1.73 & 7.20 & 136.5 & 35.79 \\
\hline 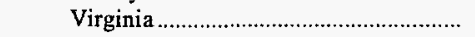 & 37.4 & 13,700 & .84 & 5.84 & 177.9 & 48.75 \\
\hline Washington & .1 & 10,800 & .73 & 14.00 & 168.0 & 36.29 \\
\hline 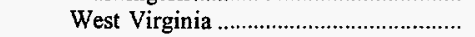 & $2,273.0$ & 12,544 & .81 & 10.68 & 152.5 & 38.26 \\
\hline Wyoming & $3,390.9$ & 8,471 & .38 & 5.55 & 123.1 & 20.86 \\
\hline 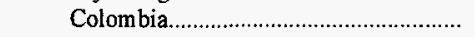 & 523.1 & 11,996 & .61 & 6.18 & 153.2 & 36.75 \\
\hline 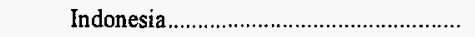 & 223.5 & 9,623 & .21 & 1.13 & 157.9 & 30.38 \\
\hline Venezuela & 459.7 & 12,849 & .83 & 6.75 & 154.2 & 39.63 \\
\hline 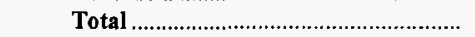 & $12,629.7$ & 11,101 & .95 & 7.34 & 146.1 & 32.43 \\
\hline \multicolumn{7}{|l|}{ Year to Date } \\
\hline Alabama & 109.0 & 12,605 & 1.11 & 10.32 & 161.8 & 40.79 \\
\hline Colorado & 624.6 & 10,470 & .36 & 5.68 & 140.4 & 29.40 \\
\hline 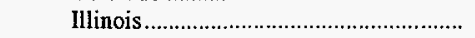 & $2,796.2$ & 12,015 & 2.00 & 7.80 & 161.0 & 38.68 \\
\hline Indiana & 32.2 & 10,891 & 2.98 & 10.09 & 123.6 & 26.92 \\
\hline 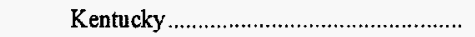 & $5,045.6$ & 12,441 & 1.43 & 8.51 & 158.0 & 39.32 \\
\hline 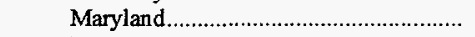 & 56.7 & 13,070 & 1.47 & 8.61 & 146.3 & 38.25 \\
\hline 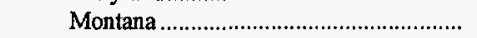 & $1,606.0$ & 9,408 & .38 & 4.51 & 147.4 & 27.73 \\
\hline Ohio & 145.0 & 12,203 & 3.29 & 10.87 & 92.7 & 22.62 \\
\hline 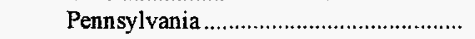 & $1,096.4$ & 13,109 & 1.72 & 7.38 & 137.1 & 35.95 \\
\hline 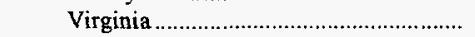 & 65.2 & 13,512 & .82 & 6.71 & 175.4 & 47.40 \\
\hline 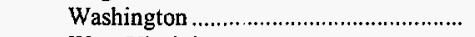 & .9 & 10,800 & .73 & 66.06 & 165.5 & 35.74 \\
\hline 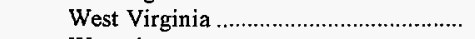 & $4,717.2$ & 12,587 & .90 & 10.40 & 152.3 & 38.35 \\
\hline 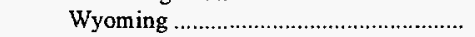 & $6,672.5$ & 8,471 & .39 & 5.55 & 124.2 & 21.04 \\
\hline 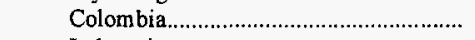 & $1,203.9$ & 12,026 & .62 & 6.42 & 157.5 & 37.88 \\
\hline 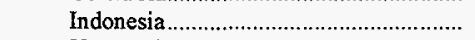 & 297.3 & 9,594 & .25 & 1.12 & 159.2 & 30.55 \\
\hline 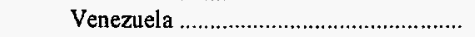 & $1,070.7$ & 12,999 & .74 & 6.49 & 156.8 & 40.75 \\
\hline Total & $25,539.5$ & 11,146 & .97 & 7.39 & 148.0 & 32.98 \\
\hline
\end{tabular}

1 Data reported on quality of coal as received.

2 Average cost data on coal delivered to Tampa Electric, Big Bend plant from the New Orleans transfer facility do not include the transportation cost of approximately $\$ 5$ per short ton from New Orleans to Tampa.

Notes: Total may not equal sum of components because of independent rounding. Only plants that have received imported coal since January 1 , 1990, are included.

Source: Federal Energy Regulatory Commission, FERC Form 423, "Monthly Report of Cost and Quality of Fuels for Electric Plants." 